\title{
THREE-DIMENSIONAL SELF-ASSEMBLED HYBRID SILICON NANOSTRUCUTRES FOR HIGH SENSITIVITY SERS CHEMICAL AND BIOSENSING APPLICATIONS
}

\author{
by \\ Jeffery Alexander Powell, B.Sc., M.Sc. \\ McMaster University, Hamilton, Ontario, Canada, 2010 \\ Trent University, Peterborough, Ontario, Canada, 2013
}

A dissertation presented to Ryerson University

in partial fulfillments of the

requirements for the degree of

Doctor of Philosophy

in the Program of

Mechanical and Industrial Engineering

Toronto, Ontario, Canada, 2017

(C) Jeffery Alexander Powell 2017 


\section{Author's Declaration}

I hereby declare that I am the sole author of this dissertation. This is a true copy of the dissertation, including any required final revision, as accepted by my examiners.

I authorize Ryerson University to lend this dissertation to other institutions or individuals for the purpose of scholarly research.

I further authorize Ryerson University to reproduce this dissertation by photocopying or by other means, in total or in part, at the request of other institutions or individuals for the purpose of scholarly research.

I understand that my dissertation may be made electronically available to the public. 


\title{
Abstract \\ Three-dimensional self-assembled hybrid Si nanostructures for high sensitivity SERS chemical and biosensing applications
}

\author{
(C) Jeffery Alexander Powell 2017 \\ Doctor of Philosophy \\ In the Program of Mechanical and Industrial Engineering, Ryerson University
}

Raman spectroscopy is a powerful tool for detection of chemical and bioanalytes but lacks enhancement required to detect these analytes at the ultrahigh sensitivity needed for many applications. Surface enhanced Raman Scattering is a technique by which an analyte signal can become greatly enhanced and, near single molecule sensitivity, is achievable. Currently, SERSbased detection platforms currently rely on noble metal nanostructures as primary enhancing sources for the detection of chemical and bioanalytes but have significant limitations in terms of reproducibility and biocompatibility. Recent research has shown that semiconductors have the ability to exhibit SERS enhancing characteristics that can potentially supplant the use of noble metals without the limitations associated with noble metal nanomaterials. This thesis presents, the generation of three-dimensional self-assembled hybrid silicon nanostructures though a laser-ion plume formation mechanism. These Si nanostructures exhibit high sensitivity SERS enhancement characteristics which can be applied for chemical and biosensing applications.

In this thesis, the Raman enhancing characteristics of the hybrid Si nanostructures are examined and correlated to the unique physical morphology and material chemistry of these nanostructures. These Si nanostructures are shown to be comprised of individual Si nanospheroids that have fused to form a highly 3D nanoweb-like self-assembled nanostructures. It is also shown that these 
nanospheroids are composed of both amorphous and polycrystalline sub-regions, which can only be generated within an ion-plume formed by a femtosecond pulsed laser. By programming the laser, the nanostructure morphology and hybrid nature can be manipulated and optimized. These Si nanostructures are shown to be highly sensitive as SERS platforms for chemical analytes. In addition, it is shown that with the application of noble metal nanoparticles on the surface of the 3D hybrid silicon nanowebs structures, an additional enhancement boost can be optimized for the detection of chemical molecules. With this, the dual contribution to the SERS sensitivity from both the primary Si nanostructures and the secondary noble metal nanostructures can be used to detect the presence of a biomolecule analyte is shown.

To delve deeper into how these hybrid Si nanostructures cause SERS enhancement of bioanalytes, the Si ion interactions within the laser-ion plume were manipulated to induce quantum-scale defects within the hybrid Si nanospheroids. By creating both an inert and oxygenated laser-ion plumes the formation of sub-nanograins within the nanospheroids and sub-nanovoids on the nanospheroid surface is shown to significantly enhance the detection of bioanalyte signal for multiple biomolecules which act as signals for various diseases. Based on the results in this thesis, it has been proven that Si-based nanostructures have the capacity to be used as sole SERS enhancing sources for chemical and biomolecule analytes. 


\section{Table of Contents}

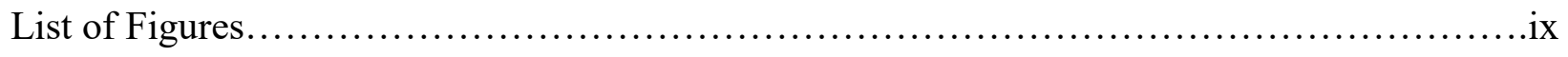

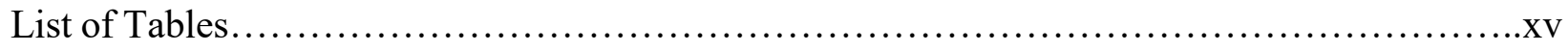

List of Abbreviations.....................................................................

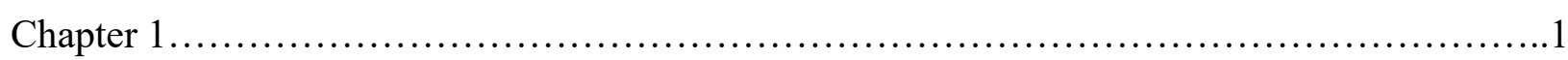

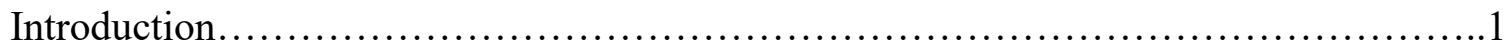

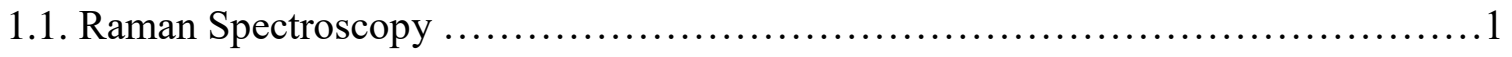

1.2. Surface Enhanced Raman Scattering (SERS) ................................ 2

1.2.1. Electromagnetic Theory ......................................... 3

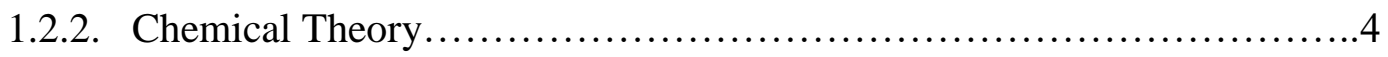

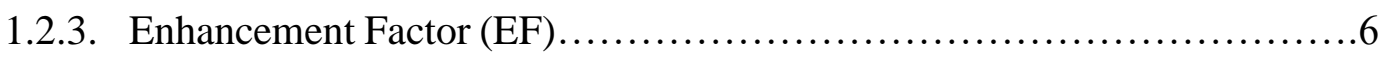

1.3. Current State of SERS Chemical and Biosensing.............................. 9

1.3.1. Noble Metal Nanostructure-based SERS detection and applications.........9

1.3.2. Semiconductor Nanostructure-based SERS sensing and applications......13

1.4. Nanostructure formation using ultrafast femtosecond pulsed laser ablation..............15

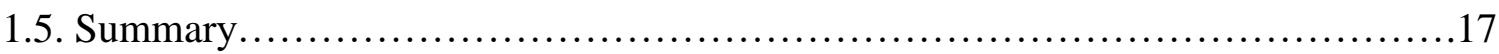

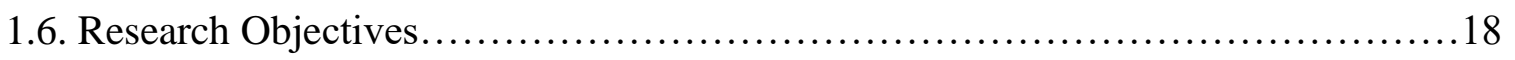

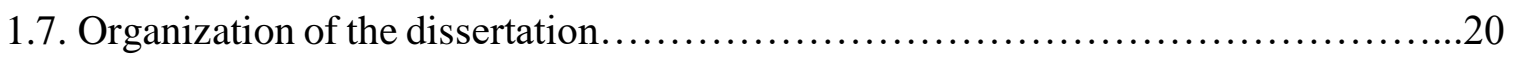

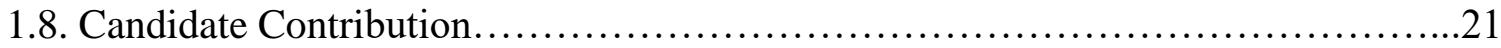

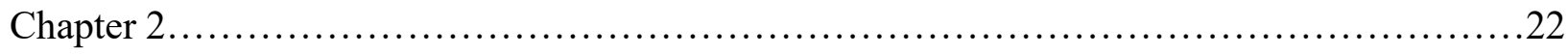

Programmable SERS active substrates for chemical and biosensing applications using amorphous/crystalline hybrid silicon nanomaterial..................................... 22

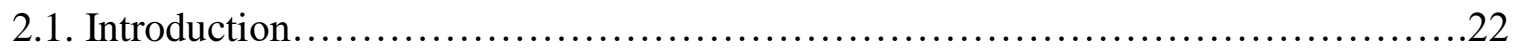

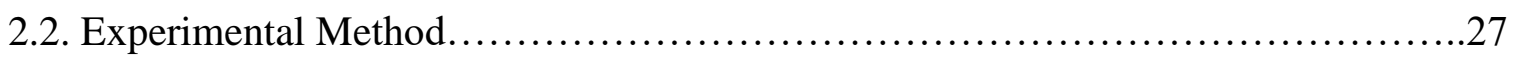

2.2.1. Laser Ablation/Raman analysis/Material characterization................27

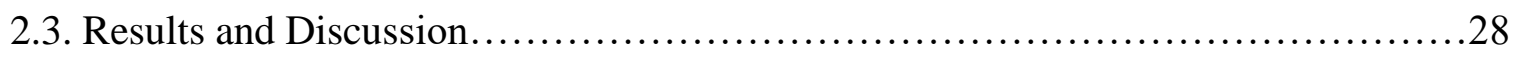

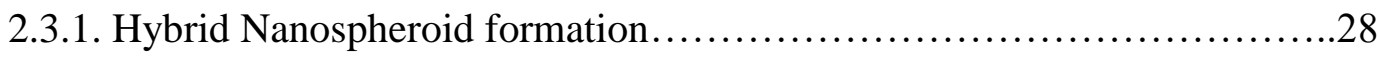

2.3.2. SERS Enhancement from hybrid nanostructures.........................35

2.3.2.1. Raman Enhancement from hybrid nanostructures................35

2.3.2.2. Raman Results for bare substrates.............................36 
2.3.2.3. Raman Results for dye-coated substrates....................36

2.3.4. Semiconductor SERS enhancement...............................40

2.3.4.1. SPR contributors: Hybrid Structure enhancement.............40

2.3.4.2. SPR contributors: Nanospheroid Size and Nanogap

Enhancement....................................................41

2.3.4.3. SPR contributors: Nanogap/Raman hot-spot enhancement.......42

2.3.5. Molecular, Charge-Transfer, and Exciton Resonance Contributors........44

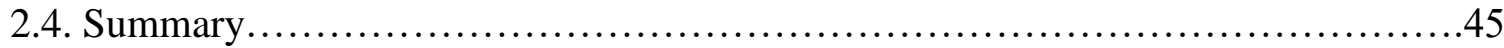

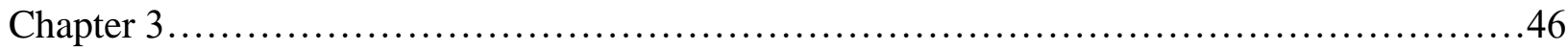

Hybridized enhancement of the SERS detection of chemical and bio-marker molecules through $\mathrm{Au}$ nanosphere ornamentation of hybrid amorphous/crystalline Si nanoweb nanostructure biochip

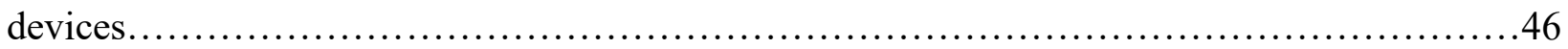

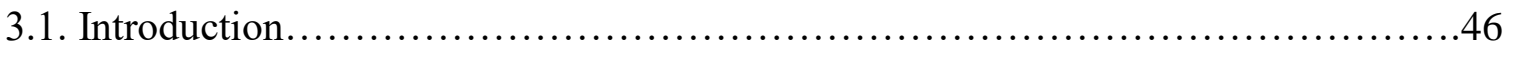

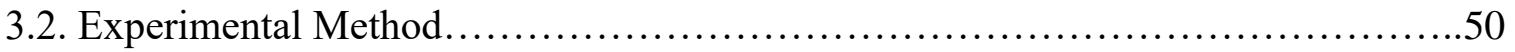

3.3. Results and Discussion.................................................... 52

3.3.1. Silicon Laser-Ion Plume Nanospheroid Biosensing Material Formation and

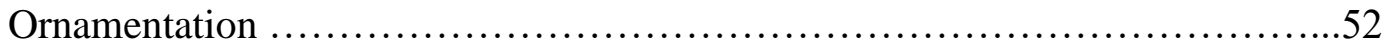

3.3.2. SERS augmentation through an Ornamentation Process..................54

3.3.3. Semiconductor and Metallic SERS Enhancement .....................57

3.3.4. SERS Biochip Enhancement Efficiency Analysis using CV dye..........61

3.3.5. Analysis for Application of SERS biochip enhancement using bio-label

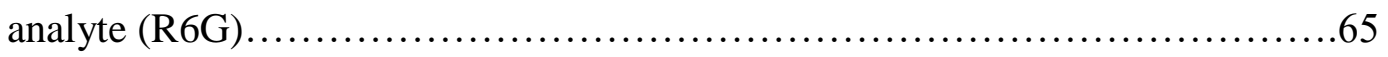

3.3.6. Influence of Ornamentation time on biochip enhancement efficiency.....67

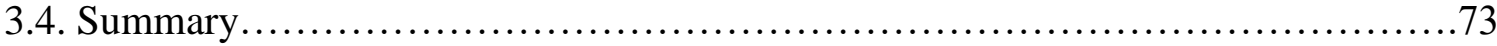

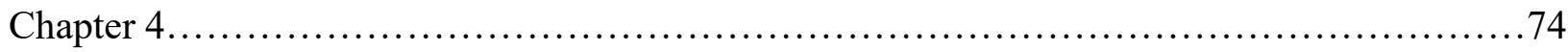

SERS detection of protein biomolecule on polymorphic Si interconnected nanospheroid nanocore network platform with secondary noble metal nanosatellite boosting mechanism...............74

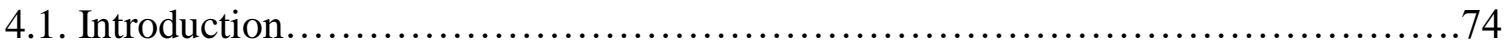

4.2. Experimental Method ................................................ 79

4.3. Results and Discussion................................................. 80

4.3.1. Nanocore superstructure Formation and SERS Enhancement.............85 
4.3.2. SERS Biomolecular Enhancement.....................................90

4.3.2.1. Polymorphic Si nanocore SERS Biosensing Efficiency........... 90

4.3.2.2. SERS Biomolecular Enhancing using Au and AuPd Nanosatellite

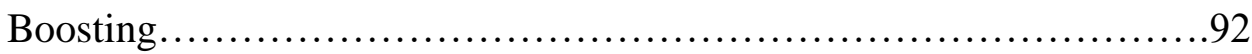

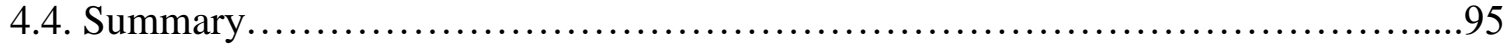

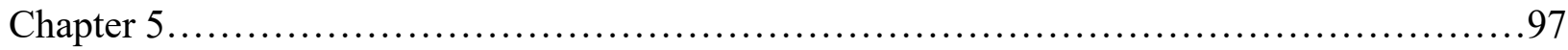

Towards Universal Si SERS-based nanobioprobe materials through engineering quantum-scale defects within self-assembled Si-only disordered 3D nanomesh structures ......................97

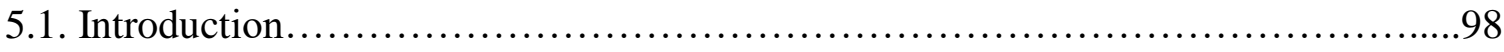

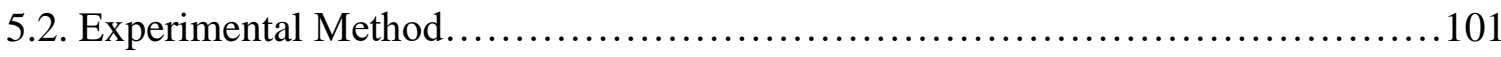

5.3. Results and Discussion.................................................... 102

5.3.1. Nanomesh formation by multi-photon ionization of solid Si substrate.............102

5.3.2. Characterization of disordered Si nanomeshes.................................104

5.3.3. Analysis of Quantum-scale defects...................................... 107

5.3.3.1 Sub-nanograin Disorder...........................................108

5.3.3.2. Surface Nanovoid disorder..........................................110

5.3.4. SERS Biosensing Activity of Nanomesh...................................111

5.3.5 Bioanalyte Enhancement efficacy of quantum scale disordered nanomeshes.......113

5.3.6. Towards Universal sensing with disordered Si nanomesh......................119

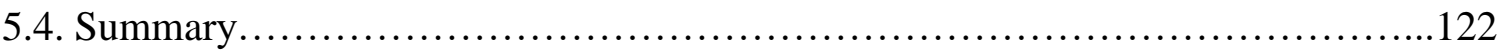

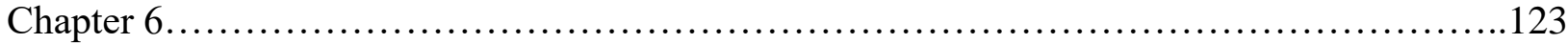

Conclusions and Recommendations for Future Research................................123

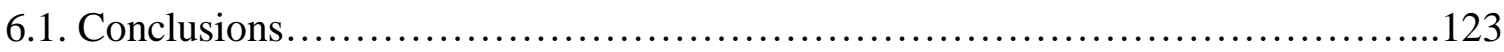

6.2. Future Research...............................................................

6.2.1. Au-Si alloy-like nanostructure formation by laser ionization for dual SPRCT SERS biomolecule enhancement..................................... 125

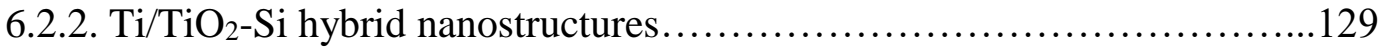

6.2.3. Multisource $\mathrm{Au}$ boosting of $\mathrm{Si}$ nanostructures: internal and external

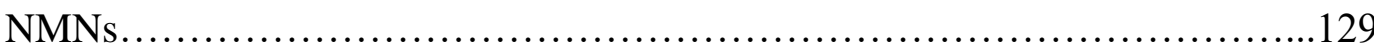

6.2.4. $\mathrm{Cu} / \mathrm{CuO} \mathrm{Si}$ nanohybrid structures..................................129 
6.2.5. DNA detection using Si-based nanostructure: Labelled and Label-free SERS

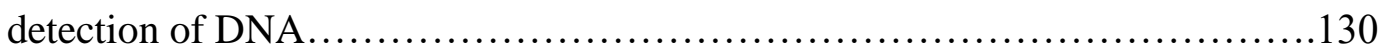

References........................................................................ 131

Appendix A................................................................... 161

A.1. Published Articles..........................................................

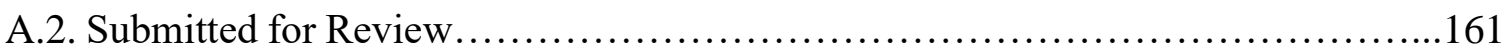

A.3. Pending Submission Upon Approval.........................................161 


\section{List of Figures}

Figure 1-1: Elastic and Inelastic Photon Scattering.......................................2

Figure 1-2: Surface Plasmon Resonance..............................................

Figure 1-3: Charge-Transfer Resonance..............................................

Figure 1-4: Ag nanorod array for SERS detection of $\mathrm{Hg}^{2+}$ ions (Courtesy [1-

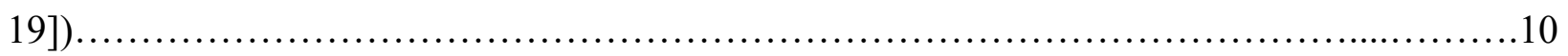

Figure 1-5: Au nanopopcorn-like particles for SERS detection of chloroptrifos insecticide

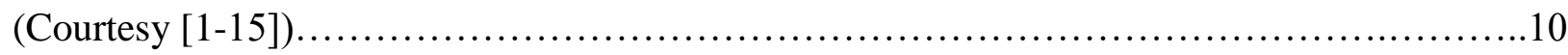

Figure 1-6: 3D titanium nanosheets with Ag nanoparticles for SERS detection of toxic pesticide

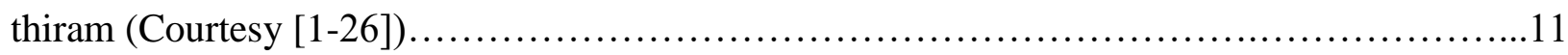

Figure 1-7: AuNP decorated Si nanowires for SERS detection of4, 4'-Bipyridine (Courtesy [127]). 12

Figure 1-8: Zn-dope $\mathrm{TiO}_{2}$ nanoparticles for SERS detection of levofloxacin (Courtesy[139]). 14

Figure 1-9: Sea urchin-like $\mathrm{W}_{18} \mathrm{O}_{49}$ nanostructures for SERS detection of R6G dye (Courtesy [140])

Figure 1-10: Ultrafast femtosecond pulsed laser Ionisation and nanostructure formation.... .16

Figure 2-1: : a) Overall schematic of Nanoweb formation and b) SERS enhancement spectra of R6G dye on hybrid Si nanoweb and c) SERS enhancement spectra of CV dye on hybrid $\mathrm{Si}$ nanoweb .26

Figure 2-2: a) Schematic representation of ion plume nanospheroid formation mechanism, b) representation of nanoweb layer on substrate surface, c) SEM images of nanoweb layer 
Figure 2-3: HRSEM images of hybrid amorphous/crystalline silicon nanoweb structures showing a) 3D nanoweb morphology and b) individual fused nanospheroids with zoomed images c), d) to show detail .30

Figure 2-4: Schematic representation of theoretical hybrid amorphous/crystalline nanospheroid formation mechanism.

Figure 2-5: XRD Spectra for nanoweb structures. .32

Figure 2-6: a) Schematic showing hybrid nature of nanospheroids with HRSEM and HRTEM images showing nanoweb structure and b) amorphous/crystalline grains within nanospheroid and Rietveld spectra for nanoweb created at c) high peak power and d) lower peak power. .33

Figure 2-7: a) Schematic of Raman enhancement of bare Si nanowebs, b) Raman spectra silicon peaks on Si nanoweb and $\mathrm{Si}$ wafer substrate, Raman spectral peak $520 \mathrm{~cm}^{-1}$ for $\mathrm{Si}$ on $\mathrm{Si}$ nanowebs formed at c) low peak power and d) high peak power, and e) EF values at $520 \mathrm{~cm}^{-1}$ for $\mathrm{Si}$ on Si nanowebs as a function of Peak Power.

Figure 2-8: a) Schematic of Raman enhancement sensing with a molecular dye on Si nanowebs, Raman spectra of b) R6G and c)CV dye on Si nanoweb and Si wafer substrate, Raman spectra for d) R6G and e) CV on Si nanowebs for each laser ion plume condition. .38

Figure 2-9: EF values for R6G and CV dyes on Si nanoweb structures at $10^{-3} \mathrm{M}$ and $10^{-6} \mathrm{M}$ concentrations as a function of peak power.

Figure 2-10: a) HRTEM images of Hybrid Amorphous/Crystalline nanospheroids, and b) a schematic of Grain Boundary Raman Scattering within a Single Crystalline Nanospheroid, an Amorphous Only Nanospheroid, a Polycrystalline Nanospheroid and a Hybrid Amorphous/Crystalline Nanospheroid.

Figure 2-11: a) Nanospheroid size distribution and b) Enhancement Factor as a function of peak power at $0.431 \mathrm{MW}, 2.88 \mathrm{MW}$ and $18.7 \mathrm{MW}$ .42

Figure 2-12: a) Nanogap size distribution and b) Enhancement Factor as a function of peak power for nanowebs created at $0.431 \mathrm{MW}, 2.88 \mathrm{MW}$ and $18.7 \mathrm{MW}$ 
Figure 3-1: Overall schematic of SERS-active biosensing nanomaterial study; a hybrid Si nanospheroid network ornamented with Au nanospheres as a SERS biochip sensor

Figure 3-2: a) The hybrid crystalline/amorphous Si nanoweb formation by laser-ion plume formation mechanism and b) the deposition of Si nanospheroids onto Si wafer surface.....

Figure 3-3: a) Ornamentation of hybrid Si nanoweb with Au nanospheres using physical deposition method with b) an HRTEM image of ornamented nanoweb material. .55

Figure 3-4: a) Raman spectra of ornamented hybrid Si nanoweb materials and b) EF values of each SERS biochip nanomaterial compared to bare Si wafer substrate

Figure 3-5: a) Schematic of SPR enhancement phenomenon and b) charge-transfer resonance principles $.60-61$

Figure 3-6: Schematic of sources of analyte spectra on a) $\mathrm{Si}$ nanoweb structures (top), on b) $\mathrm{Si}$ substrate (bottom), c) Au nanoweb structures (top) and on d) Au ornamented on Si substrate (bottom).

Figure 3-7: a) Schematic of experimental study of CV chemical analyte on ornamented hybrid $\mathrm{Si}$ nanoweb biochip structure with b) Raman spectra of CV on Si nanoweb structure and c) ornamented Si nanostructure, and d) associated EF values $63-64$

Figure 3-8: a) Diagram of ornamented hybrid Si nanoweb coated with R6G bio-label molecule and b) Raman spectra of each bare ornamented hybrid Si nanoweb and c) spectra of R6G biolabel on each nanoweb biochip structure .66

Figure 3-9: a) Representation of influence of OT on amount of Au nanospheres ornamented on hybrid-Si nanoweb biochip structure, b) HRTEM images of short and long OT nanowebs, c) Raman spectra of R6G on each nanoweb biochip structure and d) EF values associated with each biochip structure .68

Figure 3-10: a) Schematic showing how OT time causes nano-masking and nano-shielding with b) HRTEM images of bare, short OT and long OT nanoweb structures. 
Figure 3-11: a) XPS spectra for short, moderate and long OT nanoweb biochip structures with b) percentage values of $\mathrm{Si}, \mathrm{O}$ and $\mathrm{Au}$ at each OT and c) HRTEM images of short OT and long OT nanoweb biochip structures.

Figure 4-1: Raman spectra with TEM and schematic diagrams of a) Si nanocore enhancement of $520 \mathrm{~cm}^{-1}$ peak at $532 \mathrm{~nm}$ with spectra of GSH on Si nanocores at $532 \mathrm{~nm} \mathrm{b)} \mathrm{Si} \mathrm{nanocores} \mathrm{with} \mathrm{Au}$ nanosatellites showing enhancement of $520 \mathrm{~cm}^{-1} \mathrm{Si}$ peak and spectra of GSH @ $523 \mathrm{~nm}$ and c) Si nanocores with AuPd nanosatellites showing enhancement of $520 \mathrm{~cm}^{-1} \mathrm{Si}$ peak and spectra of GSH @523nm wavelength $77-78$

Figure 4-2: a) particle size distribution plots for small $(6.79 \mathrm{~nm})$ and large $(13.39 \mathrm{~nm}) \mathrm{Si}$ nanocores and b) XRD spectra of Si nanocore, Au nanosatellite deposited Si nanocore and AuPd nanosatellite deposited Si nanocore substrates. .

Figure 4-3: a) Raman spectra showing peak intensity of $520 \mathrm{~cm}^{-1}$ peak for Si wafer substrate (black), large nanocore size (blue) and small nanocore size (red) and b) the EF value at $520 \mathrm{~cm}^{-1}$ of the small and large nanocore structures relative to the Si wafer substrate c) NS spectra for $\mathrm{Si}$ nanocores, Si nanocores w/ Au nanosatellites, Si nanocores w/ AuPd nanosatellites and d) the EF of each substrate...

Figure 4-4: a) Raman Spectra of GSH biomolecule on Si wafer substrate, large Si nanocores, and small Si nanocores and b) EF values of GSH on small and large Si nanocores at micromolar (10${ }^{6} \mathrm{M}$ ) concentration c) Raman Spectra of GSH on Si nanocores w/ Au nanosatellites, and Si nanocores w/ AuPd nanosatellites and d) the associated EF values.

Figure 4-5: Size distribution plots for a) AuPd nanosatellites with b) TEM image of Si nanocore structure with AuPd nanosatellites and size distribution plot of c) Au nanosatellites with d) TEM image of Au nanosatellites on Si nanocore structure. .84

Figure 4-6: a) UPLIP formation process and b) physical deposition of Au and AuPd nanosatellites onto nanocore superstructure surface .86

Figure 4-7: Two-factor contribution to SERS enhancement, the primary boost from the Si nanocores and the secondary boost from the noble metal nanosatellites. 
Figure 4-8: a) The boosting efficiency of the $1450 \mathrm{~cm}^{-1} \mathrm{GSH}$ peak with Au and AuPd nanosatellites on $6.79 \mathrm{~nm}$ and $13.36 \mathrm{~nm}$ Si nanocores and b) schematic showing occupation of Raman interaction volume with Au and AuPd nanosatellites.

Figure 5-1: : Overall Schematic showing a) ion interaction amendmentation to form inert or oxygenated ion-plumes, b) types of engineered quantum scale defects within NO structure, c) application of biomolecule and subsequent SERS enhancement spectra .98

Figure 5-2: a) femtosecond laser ionization of a defect-free silicon wafer, NO ejection from ion plume, NOs fuse together on $\mathrm{Si}$ wafer surface to form 3D nanomesh b) images of nanomesh morphology and hybrid Si NOs.

Figure 5-3: a) HRTEM analysis of quantum scale defects within NO formed under inert-ion plume conditions and associated b) XRD spectra, c) NO size distribution d) calculated crystallite size and e) calculated residual stress .105

Figure 5-4: a) HRTEM analysis of quantum scale defects within NO formed under oxygenated ion plume conditions and associated b) XRD spectra, c) NO size distribution d) calculated residual stress. .106

Figure 5-5: TEM and HRTEM images of disordered Si nanomesh structures formed under a) inert ion-plume conditions, b) oxygenated ion-plume conditions and the observed quantum scale defects c) sub-nanograin disorder within the $\mathrm{NO}$ structure and d) sub-nanovoids on NO surface. .107

Figure 5-6: Raman spectra of the a/nc-Si nanomesh a) under oxygenated ion-plume conditions @785nm, b) oxygenated ion-plume conditions @ 532nm, c) inert ion-plume conditions @785nm and, d) inert ion-plume conditions@785nm.

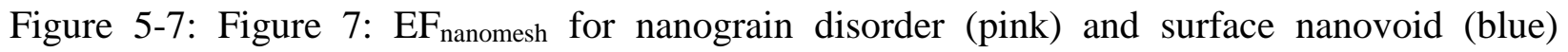
nanomeshes at a) $785 \mathrm{~nm}$ and b) $532 \mathrm{~nm}$

Figure 5-8: a) Raman spectra of GSH peaks @ 785nm, b) calculated EF values for the $1255 \mathrm{~cm}^{-1}$ peak@785nm wavelength, C) Raman spectra of GSH peaks @532nm,d)calculated EF values for the $1419 \mathrm{~cm}^{-1}$ peak @ 532nm wavelength.... 
Figure 5-9: a) Raman spectra of GSH peaks @ 785nm, b) calculated EF values for the $1255 \mathrm{~cm}^{-1}$ peak @785nm wavelength, C) Raman spectra of GSH peaks @ 532nm, d) calculated EF values for the $1419 \mathrm{~cm}^{-1}$ peak @ $532 \mathrm{~nm}$ wavelength..................................................117

Figure 5-10: Visualization of the SERS enhancing effects caused by quantum scale defects, a) sub-nanograin boundary disorder defects and b) sub-nanovoids on NO surface.......................118

Figure 5-11: Raman spectra of a) Cys, b)Trp and c) Met on nanomeshes with (pink) subnanograin boundary defects, (blue) surface sub-nanovoids and on (grey) defect free sc-Si wafer

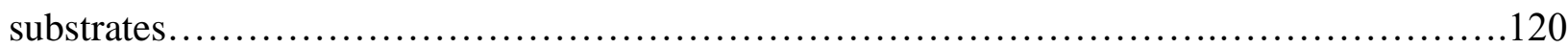




\section{List of Tables}

Table 5-1: Observed GSH peaks @785nm and @532nm with peak assignments from

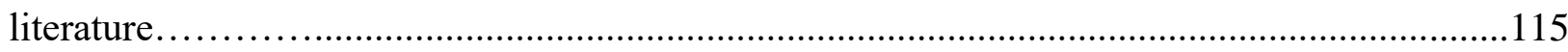

Table 5-2: Observed peaks of cysteine with peak assignments from literature .................121

Table 5-3: Observed peaks of tryptophan with peak assignments from literature.....................121

Table 5-4: Observed peaks of methionine with peak assignments from literature ...............121 


\section{List of Abbreviations}

3D - three-dimensional

CB - conduction band

CT - charge-transfer

CV - crystal violet

Cys - cysteine

EF - enhancement factor

FFT - fast Fourier transform

GSH - 1-glutathione

HOMO - highest occupied molecular orbital

HRTEM - high resolution transmission electron microscopy

LOD - limit of detection

LUMO - lowest unoccupied molecular orbital

Met - methionine

NIR - near-infrared

NMN - noble metal nanostructure

NO - nano-orb

NP - nanoparticle

NS - nanostructure

OT - ornamentation time

R6G - rhodamine 6G

SEM - scanning electron microscopy

SERS - surface enhanced Raman scattering

SPR - surface plasmon resonance

S/MO - semiconductor/metal oxide

TEM - transmission electron microscopy

XRD - X-ray diffraction

UFPL -ultrafast pulsed laser

UPLIP - ultrafast pulsed laser ion-plume

VB - valence band

XPS - x-ray photoelectron spectroscopy 


\section{Chapter 1}

\section{Introduction}

\subsection{Raman Spectroscopy}

Many techniques currently exist for identifying and classifying inorganic and organic materials based on a unique spectral signature whether it be based on electrochemical ${ }^{[1-1]}$, piezoelectric ${ }^{[1-2]}$, pyroelectric ${ }^{[1-3]}$ or optical $^{[1-4]}$ phenomenon. Optical sensing methods, chiefly Raman sensing techniques attract a large amount of research due to its ability to detect analyte non-destructively and without the need for a conductive substrate while also providing high selectivity between analytes and the potential for single molecule concentration sensitivity. Raman photon scattering is a phenomenon first discovered by C.V. Raman and K.S. Krishnan in 1928 from the observation of light of different colour being scattered through various gases and liquids. Photon scattering occurs when an incident photon impinges upon an analyte molecule and excites an electron from the ground state to an excited state. These excited electrons will then decay and emit scattered photons in one of two ways, elastically or inelastically. Elastically scattered photons will possess the same energy as the incident photons due to the electrons decaying back to the ground state; these are classified as Rayleigh scattered photons and make up the vast majority of scattered photons. Inelastically scattered photons however will have a lower energy than the incident photon due to the electron decaying to a virtual vibrational state. These emitted photons are known as Stokes, or Raman scattered photons and occur approximately 1 in $10^{12}$ scattered photons. Figure 1-1 shows a schematic depicting the Rayleigh and Raman scattering phenomena. 

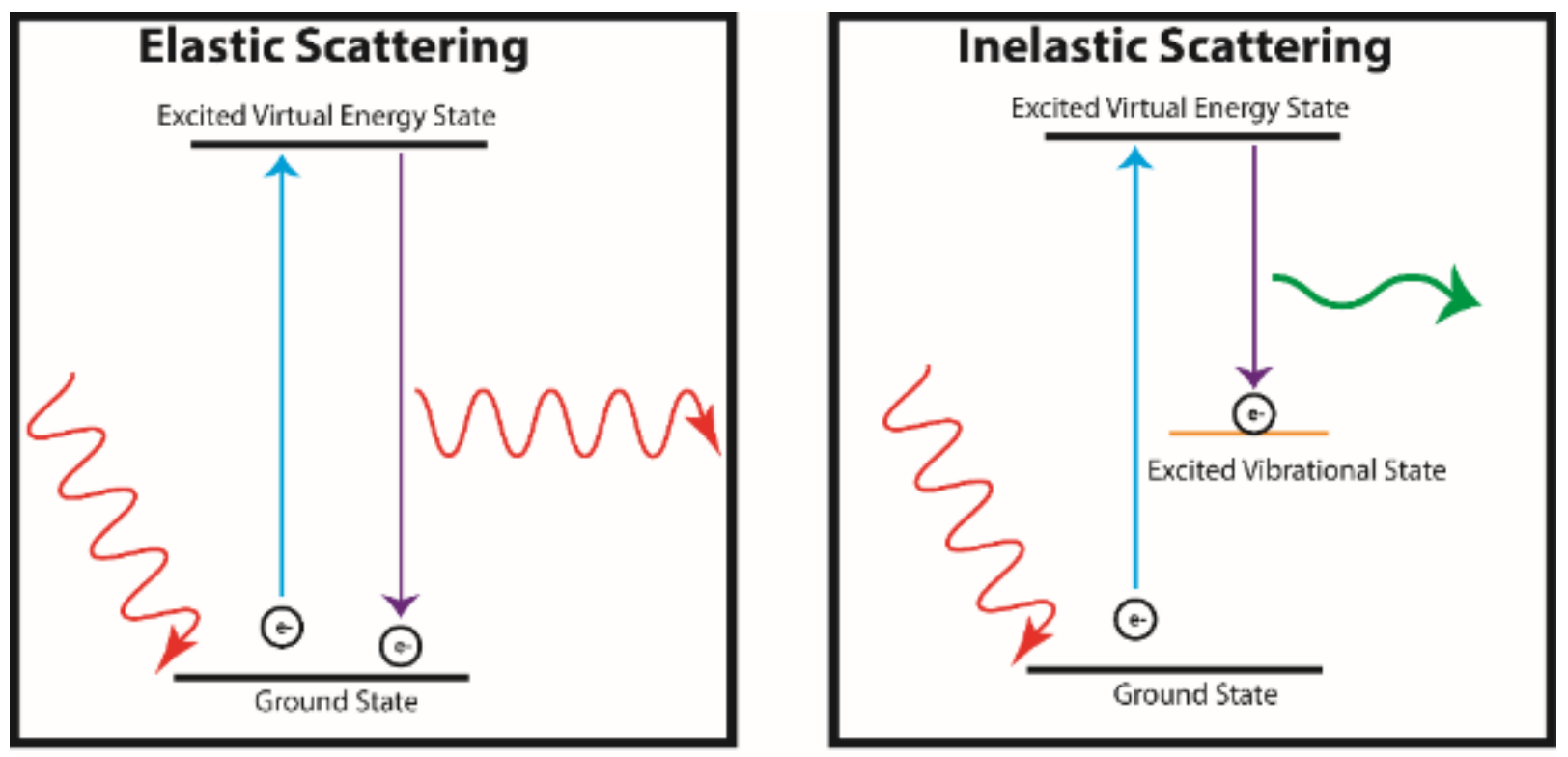

Figure 1-1: Elastic and Inelastic Photon Scattering

These Raman scattered photons will have a difference in energy from the incident photons that is dependent on the virtual vibrational state specific to the analyte, and as such will become a unique signature of the specific analyte. With Raman scattered photons it is possible to distinguish and identify different analytes based on their unique Raman spectral signature. However, as stated the frequency of Raman scattering is extremely low and can only provide a very weak signal, thus it is an impractical spectroscopic tool without a method to significantly boost the number of Raman scattered photons.

\subsection{Surface Enhanced Raman Scattering (SERS)}

The technique that has been proven to drastically increase the number of Raman scattered photons is known as surface enhanced Raman scattering or SERS. The first observed incidence of the SERS phenomenon was by Fleischmann et.al ${ }^{[1-5]}$ in 1979 when pyridine adsorbed on roughened silver substrates it exhibited significantly increased Raman signal versus an unroughened substrate. Since this observation the development of SERS sensitive materials and substrates has been a consistent and active source of research with both the development of theories that explain the underlying mechanics of SERS enhancement and the creation of SERS materials that have near single molecule sensitivity. Currently, the field of SERS sensing focuses mainly on the 
development of nanomaterials and nanostructures or varying materials and morphologies with a primary objective of achieved the lowest limit of detection possible for many different analytes. However, the underpinning theories that describe how these nanostructured materials cause SERS enhancement have been established and thoroughly proven, with some recent developments that has led to new advances in SERS sensing materials. The underlying mechanisms that govern the increase in Raman signal can be separated into two different theories, an electromagnetic theory and a chemical theory. These two mechanisms are both diverse in nature, but are not mutually exclusive and can be sources of SERS enhancement within the same material.

\subsubsection{Electromagnetic Theory}

The electromagnetic theory of SERS enhancement states that an increase in Raman scattered photons occurs due to an increase in the electromagnetic field caused by the material surface. This occurs because the incident light from the Raman laser that strikes the surface of the material, will excite localized surface plasmons. A surface plasmon is a coherent collective oscillation of electrons on the surface of a material, which creates a localized region of increased electromagnetic on the material surface. This increased electromagnetic field is maximized when the incident Raman frequency is resonant with the plasmon frequency of the material, a term called surface plasmon resonance (SPR). Figure 1-2 show a depiction of the SPR mechanism.

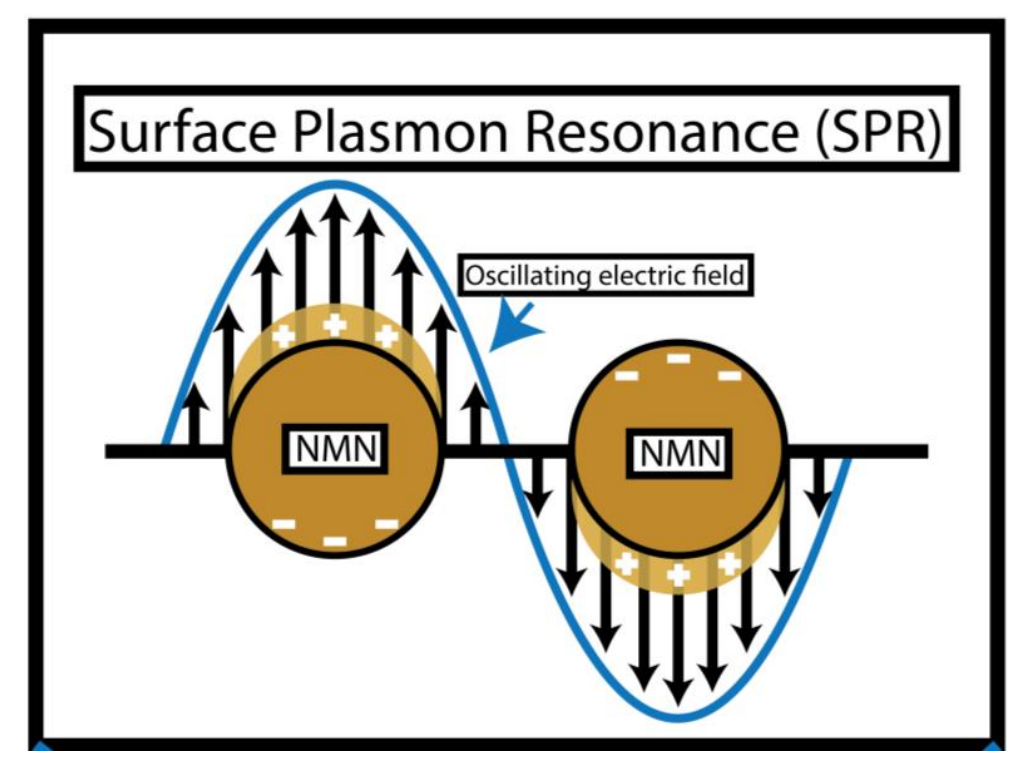

Figure 1-2: Surface Plasmon Resonance; collective oscillation of electrons on NP surface 
SPR causes SERS enhancement by both magnifying the intensity of the incident Raman laser photons and exciting the Raman modes associated with the analyte and by magnifying the intensity of the Raman scattered photons from the analyte. Some nanomaterials and nanostructures are significantly SERS active because in order to induce an increase in Raman scattering, the SPR oscillation is required to be perpendicular to the material surface rather than in-plane, otherwise scattering will not occur. Nanostructures and nanomaterials provide significant SERS enhancement because the nanoscale features confine surface plasmons to be perpendicular to the surface and since the size of these features is so small, the resulting electromagnetic field is very intense. The regions of these nanostructures that produce this strong electromagnetic field are known as "hot-spots" and are generally features of the nanostructure that are produce the most SPR such as the spacing between two adjacent nanoparticles or a nanoscale sharp edge. These hotspots are size dependant and creating a uniform spacing or size of hotspots will lead to a greater analyte enhancement ${ }^{[1-6]}$.

Due to the fact that the plasmon frequency is material dependant, not every material has the capacity to be a SERS enhancing material. Most Raman lasers are in the visible to near-infrared (NIR) wavelength ranges (480-1050nm). As a result, noble metals such as gold and silver are typically used as SERS enhancing nanomaterial because their plasmon resonance frequency correlates to this wavelength range. As such, noble metal nanostructures are widely researched as SERS sensitive materials, with many different fabrication techniques and nanostructural morphologies having been studied.

\subsubsection{Chemical Theory}

The chemical theory of SERS enhancement involves the transfer of charge between the analyte molecule and the nanostructure induced by the Raman photons. The chemical component of SERS enhancement was initially observed when molecules with very similar vibrational character exhibited significantly different intensities under identical experimental conditions. The theory of this chemical component developed by Lombardi and Birke ${ }^{[1-7,1-8]}$ states that the source for the chemical component arises from electronic transition of electrons from a nanostructure to the molecular orbitals of the analyte molecules and vice versa. If the highest occupied molecular orbital (HOMO) to lowest unoccupied molecular orbital (LUMO) of the analyte is within the Fermi 
level of a metal or the bandgap of a semiconductor, the transfer of electrons can occur with about half of the energy required within the molecule itself. As a result, electrons excited by incident Raman photons can easily transition to the molecular orbitals of the analytes causing an increase in vibrational transitions and thus more scattered Raman photons from the analyte. Transitions of electrons from the molecular orbitals of the analyte to the nanostructure also increase the Raman signal intensity by the same principle. Figure 1-3 shows the principle of chemical SERS enhancement by charge transfer resonance.

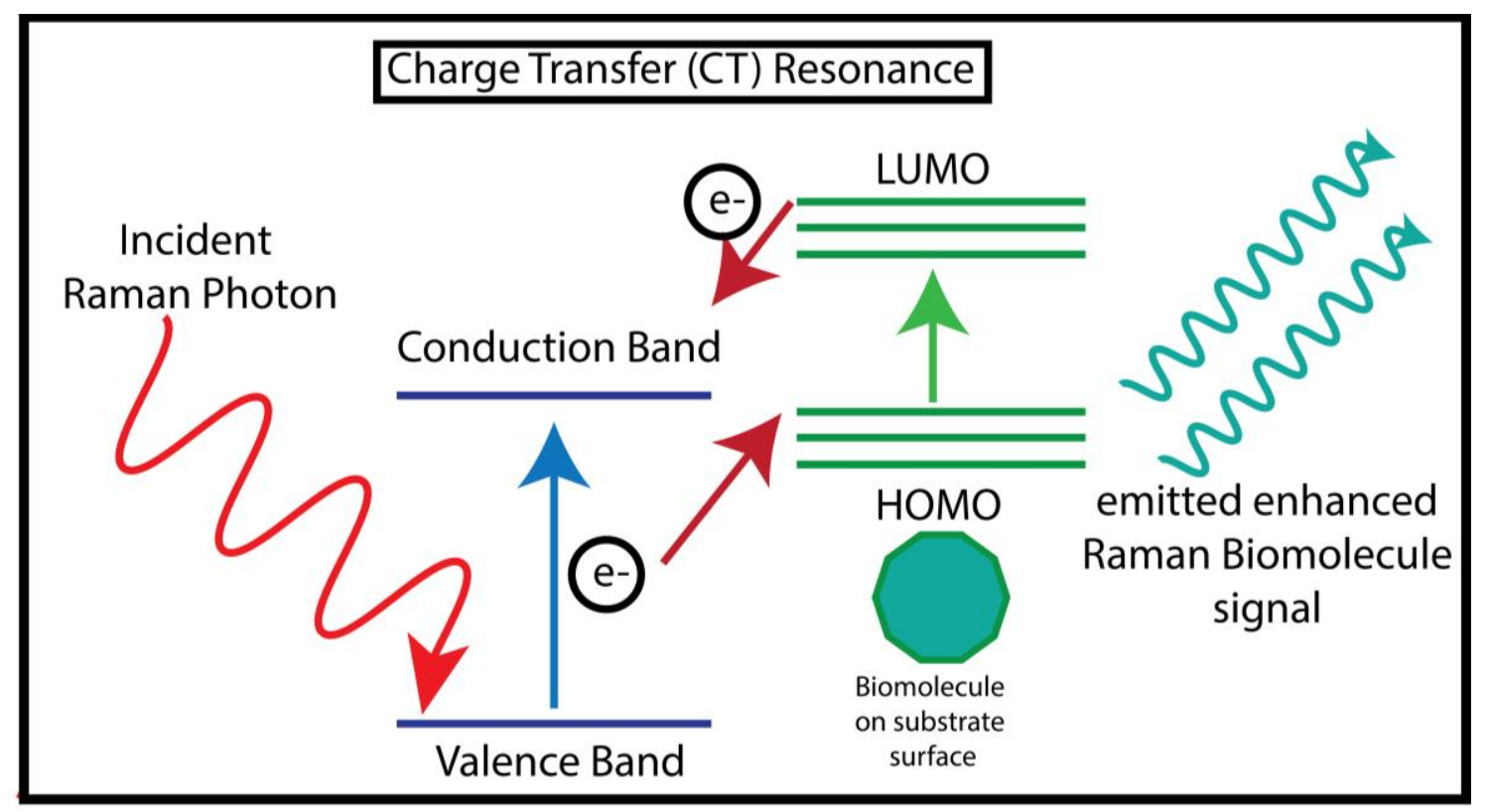

Figure 1-3: Charge-Transfer Resonance; incident photon cause electrons to be excite to higher energy states allowing for direct transfer to molecular orbitals of analyte molecules

In many cases with noble metal SERS nanostructures it is difficult to separate the electromagnetic component from the chemical component of SERS enhancement. The chemical component is largely difficult to study because any investigation into varying experimental parameters will inevitably change both components. The chemical component is often not considered due to the larger SERS contribution from SPR for these nanostructures compared to the chemical component. 


\subsubsection{Enhancement Factor (EF)}

To quantify the enhancement observed from a SERS spectra, a quantity known as the enhancement factor (EF) has been developed. The EF quantifies how the SERS spectral intensity compares to the spectral intensity of bulk substrate and indicates the level of effectiveness of the SERS substrate at producing an enhancement of the Raman signal. There are several equations that have been developed that express this intensity, the flowing equation is used as a general standard for evaluating SERS enhancement:

$$
E F=\frac{I_{\text {nanostrucutres }} / N_{\text {nanostrucutres }}}{I_{\text {bulk }} / N_{\text {bulk }}} \quad(\text { Equation 1-1) }
$$

Where $I_{\text {nanostructures }}$ is the intensity of a chosen analyte peak from the SERS spectra, $N_{\text {nanostructures }}$ is the number of molecules on the SERS substrate that contribute to the SERS enhancement, $I_{\text {bulk }}$ is the intensity of the same chosen analyte peak from the spectra obtain on the bulk substrate and $N_{b u l k}$ is the number of analyte molecules that contribute to the bulk spectra. This equation normalizes the intensity of the peaks from the respective Raman spectra by the number of analyte molecules that produce the signal. $N_{\text {nanostrucutres }}$ and $N_{\text {bulk }}$ are a necessity when analyzing an analyte solution because the number of molecules that contribute to either spectral intensity will be different on the nanostructure surface and a reference bulk surface. The determination of the $N_{\text {nanostructures }}$ and $N_{\text {bulk }}$ values are dependent on the study and the nanostructure being investigated. The complexity to which $N_{\text {nanostructures }}$ and $N_{\text {bulk }}$ are calculated will be the defining factor to which the accuracy of the EF calculation can be attributed. In the most basic sense, $N_{\text {nanostructures }}$ and $N_{\text {bulk }}$ can be defined as the concentration of the analyte solution added to the SERS or reference substrate, the volume the analyte solution occupies and the Raman laser spot area. While valid for simple measurements of two-dimensional surfaces, this does not take into account the nature of three-dimensional SERS nanostructures, which includes the adsorption of analyte molecules onto the nanostructure surfaces, the surface area of the nanostructures and Raman interaction volume. For example Cong et.al ${ }^{[1-9]}$ have determined the EF values of $\mathrm{R} 6 \mathrm{G}$ molecules on $\mathrm{W}_{18} \mathrm{O}_{49}$ nanowires using the following equations:

$$
E F=\frac{I_{S E R S} / N_{S E R S}}{I_{\text {bulk }} / N_{\text {bulk }}} \quad \text { (Equation 1-2) }
$$




$$
\begin{gathered}
N_{S E R S}=C V N_{A} A_{\text {Raman }} / A_{\text {sub }} \quad \text { (Equation 1-3) } \\
N_{\text {bulk }}=M \rho h A_{\text {Raman }} N_{A} \quad \text { (Equation 1-4) }
\end{gathered}
$$

Where $C$ is the concentration of the analyte solution, $V$ is the volume of a droplet of this solution, $A_{\text {Raman }}$ is the Raman laser spot area (based on $1 \mu \mathrm{m}$ diameter), $A_{s u b}$ is the effective area of the substrate, $M$ is the molecular weight of the analyte, $\rho$ is the density of the analyte and $N_{A}$ is Avogadro's constant. In this study, the reference for $I_{b u l k}$ is obtained from a $\mathrm{Si} / \mathrm{SiO}_{2}$. Using this equation they have been able to calculate an EF value of $3.4 \times 10^{5}$ for R6G on their nanostructures.

In the following studies, the following definition of $N_{\text {bulk }}$ first defined by Maznichenko et.al ${ }^{[1-10]}$ is used.

$$
N_{\text {bulk }}=\pi r^{2} h c N_{A} \quad(\text { Equation 1-5) }
$$

Where $r$ is the Raman laser spot radius, $h$ is the depth of field of the Raman laser, and $c$ is the concentration of the applied analyte. This calculation has been updated in the following studies to reflect an interaction volume that is a hemisphere on the wafer surface rather than a cylinder to get the following equation:

$$
N_{\text {bulk }}=2 / 3 \pi r^{3} c N_{A} \quad \text { (Equation (1-6) }
$$

The definition of $N_{\text {nanostructures }}$ also established by Maznichenko et.al ${ }^{[1-11]}$ was also used in these studies:

$$
N_{\text {nanostructures }}=S A_{\text {effective }}\left(c_{\text {ads }} \times N_{A} \frac{1000 L}{1 m^{3}}\right)^{2 / 3} \quad \text { (Equation 1-7) }
$$

Where $S A_{\text {effective }}$ is the effective area of the nanostructures that contribute to the Raman signal, $c_{a d s}$ is estimated concentration of adsorption on the nanostructure surfaces. $c_{a d s}$ is value that is determined from literature sources for similar nanostructures and analytes. To calculate the

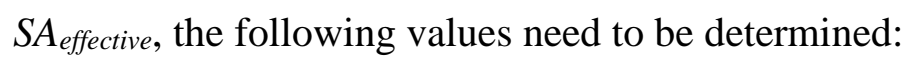

$$
m_{\text {bulk }}=2 / 3 \pi r^{3} \rho_{\text {bulk }} \quad \text { (Equation 1-8) }
$$




$$
\begin{gathered}
m_{\text {nanostructures }}=\frac{m_{\text {bulk }}}{\left(1-\frac{S A_{1}-S A_{2}}{2 / 3}\right)} \quad \text { (Equation 1-9) } \\
S A_{\text {bulk }}=2 / 3 \pi r^{3} \quad \text { (Equation 1-10) } \\
S A_{\text {nanostructures }}=\frac{2 / 3 \pi r^{3} \rho_{\text {bulk }}}{\rho_{\text {nanostrucutres }}} \quad \text { (Equation 1-11) }
\end{gathered}
$$

Where $m_{b u l k}$ is the mass of silicon that would occupy the Raman interaction volume, $\rho_{b u l k}$ is the density of silicon, $m_{\text {nanostrucutres }}$ is the mass of the Si nanostructures that would occupy the Raman interaction volume, $S A_{\text {bulk }}$ is the surface area that bulk silicon would occupy, $S A_{\text {nanostructures }}$ is the volume the Si nanostructures would occupy and $\rho_{\text {nanostructures }}$ is an estimated density of the $\mathrm{Si}$ nanostructures based on literature sources. In these studies $\rho_{\text {nanostructures }}$ is assumed to be $7.8 \mathrm{~m}^{2} / \mathrm{g}$ based on BET measurements of porous Si nanofibres ${ }^{[1-12]}$. The effective surface area $S A_{\text {effective }}$ is defined as

$$
S A_{\text {effective }}=m_{\text {nanostrucutres }} \times \rho_{\text {nanostructures }} \quad(\text { Equation } 1-12)
$$

These two approaches to calculate EF values show that EF values can vary greatly and as a result should considered to be less of an absolute measure of enhancement and more a measure of qualitative comparison. In practice, SERS substrates that are able to produce EF values $\sim 10^{4}-10^{8}$ are considered significant SERS enhancers, although some EF values as high as $\sim 10^{10}-10^{12}$ have been reported in literature; these extremely high EF values are often quoted as the theoretical maximum achievable SERS enhancement given ideal conditions. ${ }^{[1-13]}$ 


\subsection{Current Advances in SERS Chemical and Biosensing}

\subsubsection{Noble Metal Nanostructure-based SERS detection and applications}

Presently, in the field of SERS chemical and biosensing there are a large number of nanostructures being developed, and a large number of analytes are being investigated. The main objective that permeates current research is the development of a SERS nanostructure that has an extremely high enhancement efficiency and the ability to detect an analyte at very low concentration, with single molecule detection an objective in many instances ${ }^{[1-14-1-16]}$. Noble metal nanostructures (NMNs) continue to be a primary focus for many researchers which only some branching out to other SERS active semiconductor/metal oxide nanostructures. NMNs of a wide array of interesting morphologies are being generated as SERS platforms including nanorods ${ }^{[1-17,1-18]}$, nanopopcorn ${ }^{[1-}$ ${ }^{19]}$, nanoskittles ${ }^{[1-20]}$, and hollow-nanoparticles ${ }^{[1-21,1-22]}$.

For example, Song et.al ${ }^{[1-23]}$ have developed an array of silver nanorods on glass slides by oblique angle vapor deposition for the detection of $\mathrm{Hg}^{2+}$ ions using immobilized oligonucleotides. The single stranded oligonucleotides have a cyanine dye attached to one end and are bonded to the Ag nanorod array on the other and give a large cyanine Raman response. However in the presence of $\mathrm{Hg}^{2+}$ ions, the oligonucleotides transform into double stranded complexes which moves the attached cyanine dye further away from the Ag nanorod array, thus decreasing the observed Raman signal. This sensor has achieved a $0.16 \mathrm{pM}$ concentration limit of detection and has potential for use as a water pollutant detection substrate. 


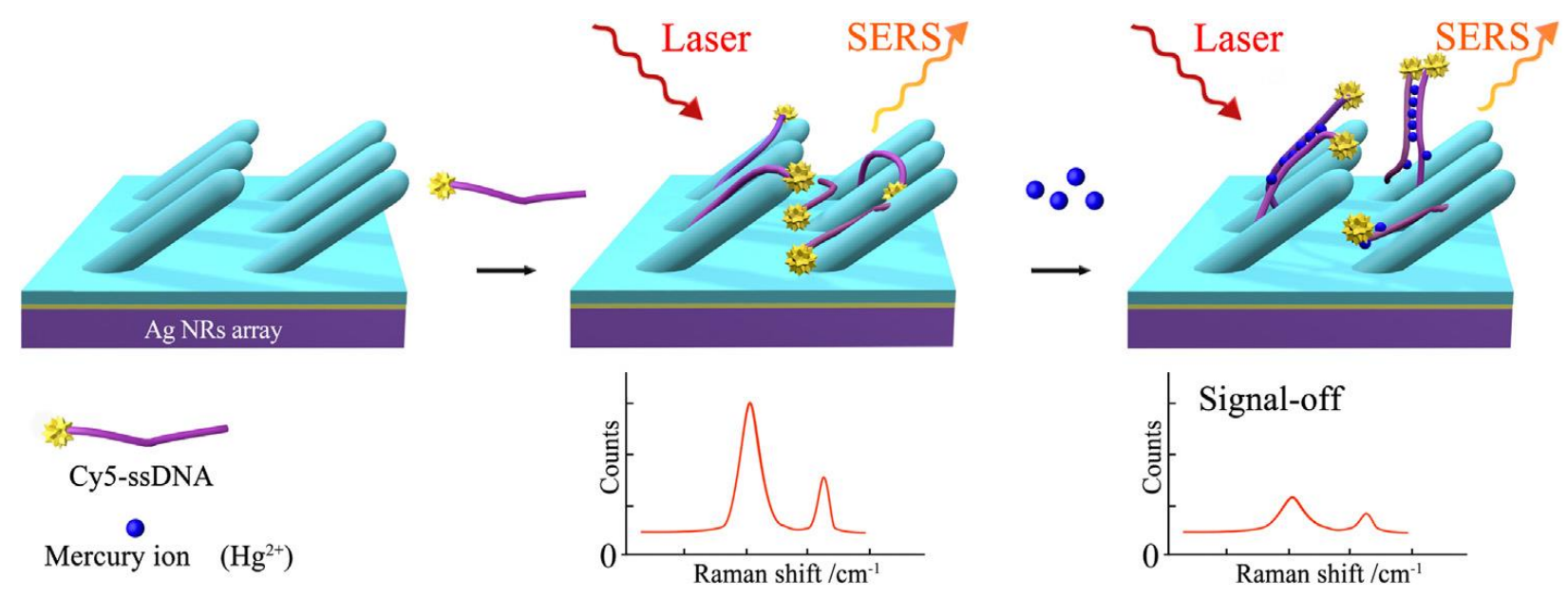

Figure 1-4: Ag nanorod array for SERS detection of $\mathrm{Hg}^{2+}$ ions (Courtesy [1-23])

Additionally, Xu et.al ${ }^{[1-19]}$ have fabricated Au nanopopcorn-like particles through seeded sol-gel chemical synthesis for the detection of a chloropyrifos-phsophorothioate organophosphate insecticide used in agricultural and pest control applications; the continued build of this insecticide has been observed to have toxic effects for nervous system function. These Au nanopopcorns are observed to have an enhancement factor of $\sim 10^{5}$ for rhodamine $6 \mathrm{G}(\mathrm{R} 6 \mathrm{G})$ dye and is observed to have a $1 \mu \mathrm{M}$ limit of detection for the chloropyrifos insecticide in aqueous solution.
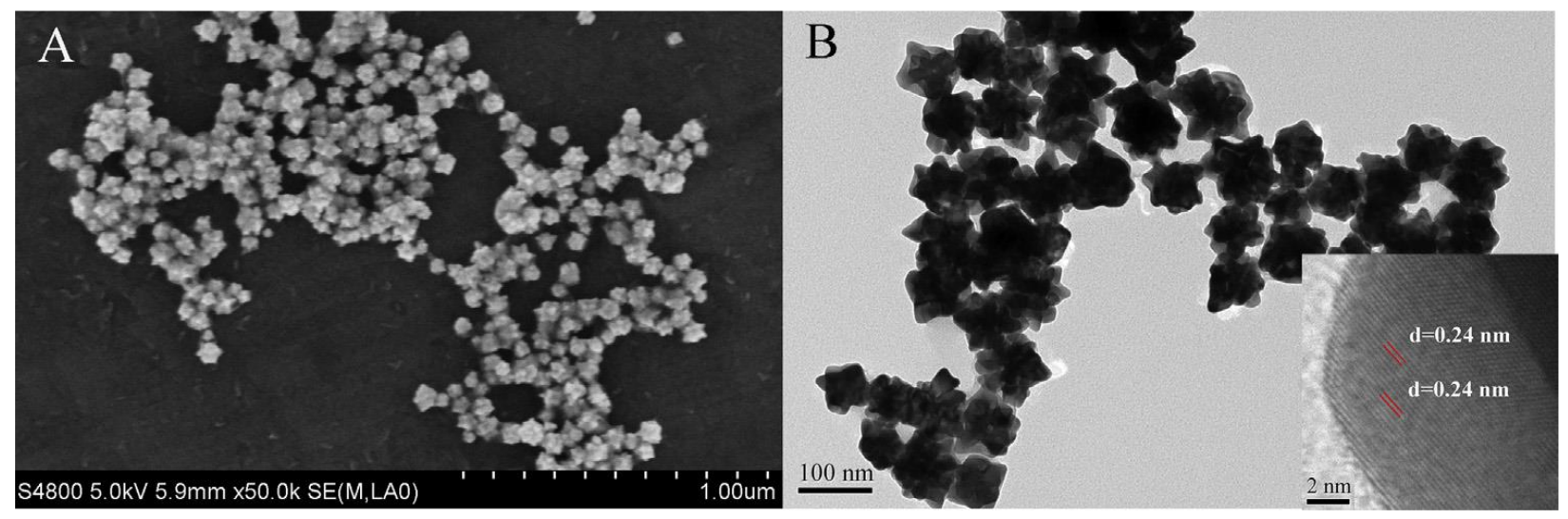

Figure 1-5: Au nanopopcorn-like particles for SERS detection of chloroptrifos insecticide (Courtesy [1-19])

An increasing amount of interest is directed towards the deposition of traditional $\mathrm{Au}$ or $\mathrm{Ag}$ nanoparticles on nanostructures of other materials such as nanowire arrays ${ }^{[1-24,1-25]}$, graphene 
sheets ${ }^{[1-26,1-27]}$, or on nanostructures formed by nanolithographic techniques ${ }^{[1-28,1-29]}$. These nanostructures act as a platform or scaffolding for the NMNs to act as primary SERS enhancing sources. For example, Dai et.al ${ }^{[1-30]}$ have fabricated a 3D titanium oxide nanostructure by hydrothermal reaction in a $\mathrm{NaOH}$ solution and ion exchange process in $\mathrm{HNO}_{3}$ solution. The subsequent titanite nanosheets had $\mathrm{Ag}$ seed deposited on the surfaces and were then illuminated by UV light to grow the $\mathrm{Ag}$ nanoparticle and anneal the substrate. The resulting $\mathrm{TiO}_{2} / \mathrm{Ag}$ nanosheet-nanoparticle structure was able to detect 4-amniothiophenol (4-ATP) up to a $10^{-7} \mathrm{M}$ concentration and was able to detect thiram, a toxic pesticide, up to $10^{-7} \mathrm{M}$ concentration in chemical form and up to $10^{-5} \mathrm{M}$ in real samples (apple skin and apple juice).

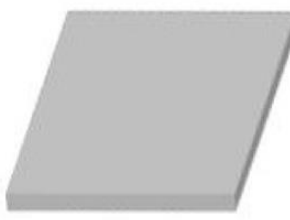

Tisubstrate

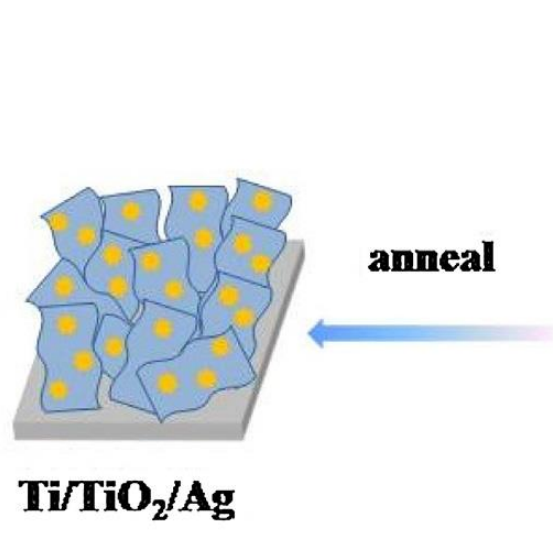

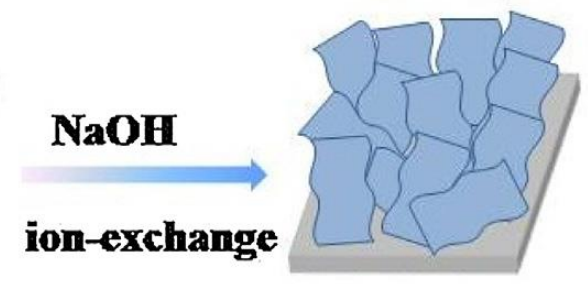

Ti/titanate

$\mathrm{AgNO}_{3}$

\section{UVirradiation}

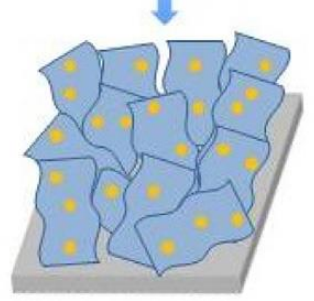

Ti/titanate/Ag

Figure 1-6: 3D titanium nanosheets with Ag nanoparticles for SERS detection of toxic pesticide thiram (Courtesy [1-30])

Li et.al ${ }^{[1-31]}$ have developed a silicon nanowire array formed by nanolithography that has been coated with a thin film of gold and has had Au nanoparticles deposited on the surfaces. These substrates are observed to have a maximum enhancement factor of $\sim 10^{6}$ of $4,4^{\prime}$-Bipyridine. 

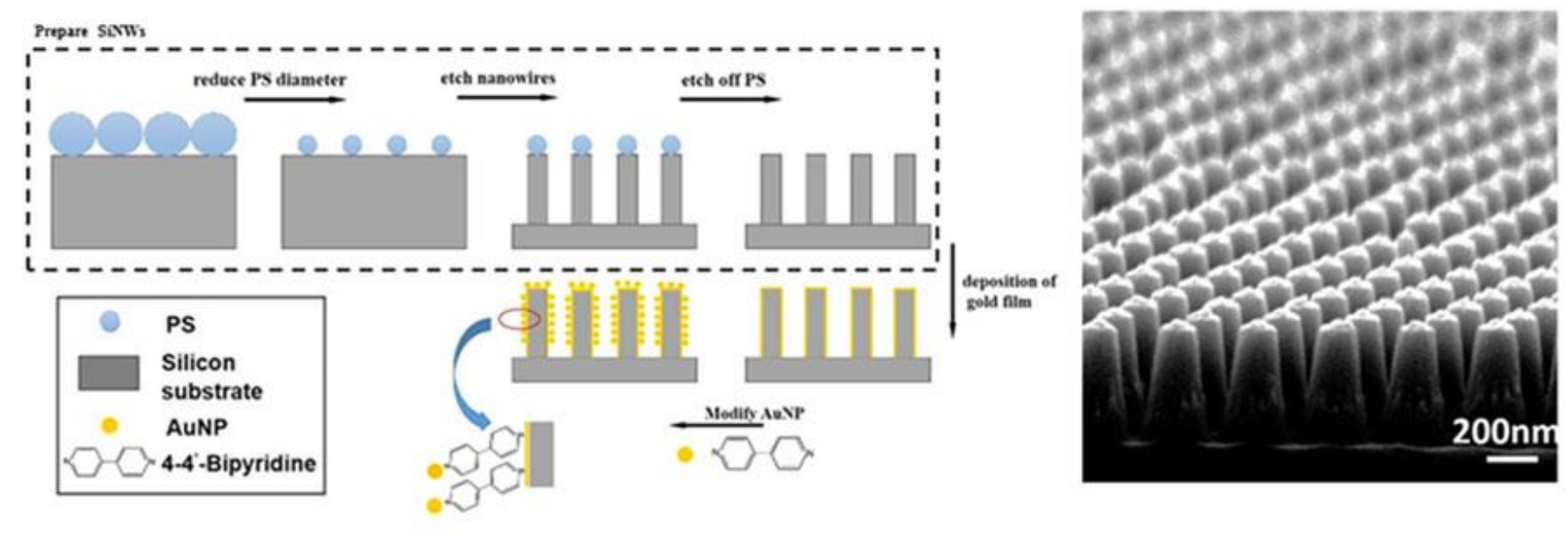

Figure 1-7: AuNP decorated Si nanowires for SERS detection of 4, 4'-Bipyridine (Courtesy [1-31])

NMNs have been used to detect a variety of biomolecules as well, ranging from colloidal Ag nanoparticles use to detect DNA present in nanopharyngeal carcinoma ${ }^{[1-32]}$ to functionalized $\mathrm{Au}$ and magnetic nanoparticles bonded to salmonella typherium bacteria ${ }^{[1-33]}$. There are however several issues that are consistent with the development of noble metals as SERS active substrates that limit their capacity as chemical and biosensors. While NMNs have been widely studied as SERS sensitive materials, there are key drawbacks associated with both their fabrication and their interactions with analytes that prevent their ubiquity. One of the major problems with using noble metals as SERS substrates is that creating a uniform, reproducible and highly sensitive sensing platform is very difficult. Since SPR is highly contingent upon how the hotspots generate an electromagnetic field, the size and arrangement of these hotspots are crucial to the level of achievable SERS enhancement. ${ }^{[1-34]}$ While nanolithographic methods can produce extremely precise nanostructure sizes and morphologies, these methods carry with them a high production costs and low throughput. Wet chemical techniques can provide nanostructures with high SERS enhancement with a low production cost and large sample size, but these methods lack controllability of nanostructure size and distribution as well as irreproducibility of SERS enhancement. Additionally, NMNs are often not biocompatible with some biomolecules which limits their use as biosensors and in some cases lead to side reactions during SERS measurement. 


\subsubsection{Semiconductor Nanostructure-based SERS sensing and applications}

Recently, semiconductor nanostructures have received a considerable amount of attention as SERS-active substrates due to observations of SERS enhancement that is comparable to NMN enhancement. The observation of Raman enhancement with a semiconductor substrate is not a recent discovery and was first reported a few years after the initial SERS observation; Yamada et.al observed an enhancement of pyridine spectra on $\mathrm{NiO}$ thin films ${ }^{[1-35]}$. Since then a large array of semiconducting materials have been observed to exhibit SERS enhancement including but not limited to, $\mathrm{ZnO}^{[1-36]}, \mathrm{TiO}_{2}^{[1-37]}, \mathrm{ZnS}^{[1-38]}, \mathrm{CuO}^{[1-39]}, \mathrm{Fe}_{3} \mathrm{O}_{4}^{[1-40]}, \mathrm{CdTe}^{[1-41]}$, and $\mathrm{PbS}^{[1-42]}$. Semiconductors exhibit SERS enhancement through the charge transfer mechanism and as a result most semiconductor nanostructures do not exhibit plasmon resonance effects. This is due to the fact that most semiconductor materials are plasmonically inactive in the visible to NIR wavelength range. This results in a reliance on the CT mechanism to produce SERS enhancement for these materials. In the last few years there have been reported semiconductor nanostructures that have been developed that are able to achieve enhancement factor values that rival EF values observed in NMNs.

For example, Yang et.al ${ }^{[1-43]}$ have fabricated $\mathrm{Zn}$-doped $\mathrm{TiO}_{2}$ nanoparticles that have the ability to detect and enhance the spectra of levofloxacin drug molecules which can be found in animal food and environmental sources which inhibits DNA replication. They were fabricated these Zn doped $\mathrm{TiO}_{2}$ nanoparticles by sol hydrothermal synthesis and dispersed in ethanol solution and were able to optimize the synthesis of the nanoparticle to achieve a detection limit of $\sim 10^{-7} \mathrm{M}$ for the levofloxacin molecule. They concluded that the charge transfer mechanism was responsible for the observed enhancement because the defect structure and bandgap-to-HOMO/LUMO energy difference made electrons transitions possible. 


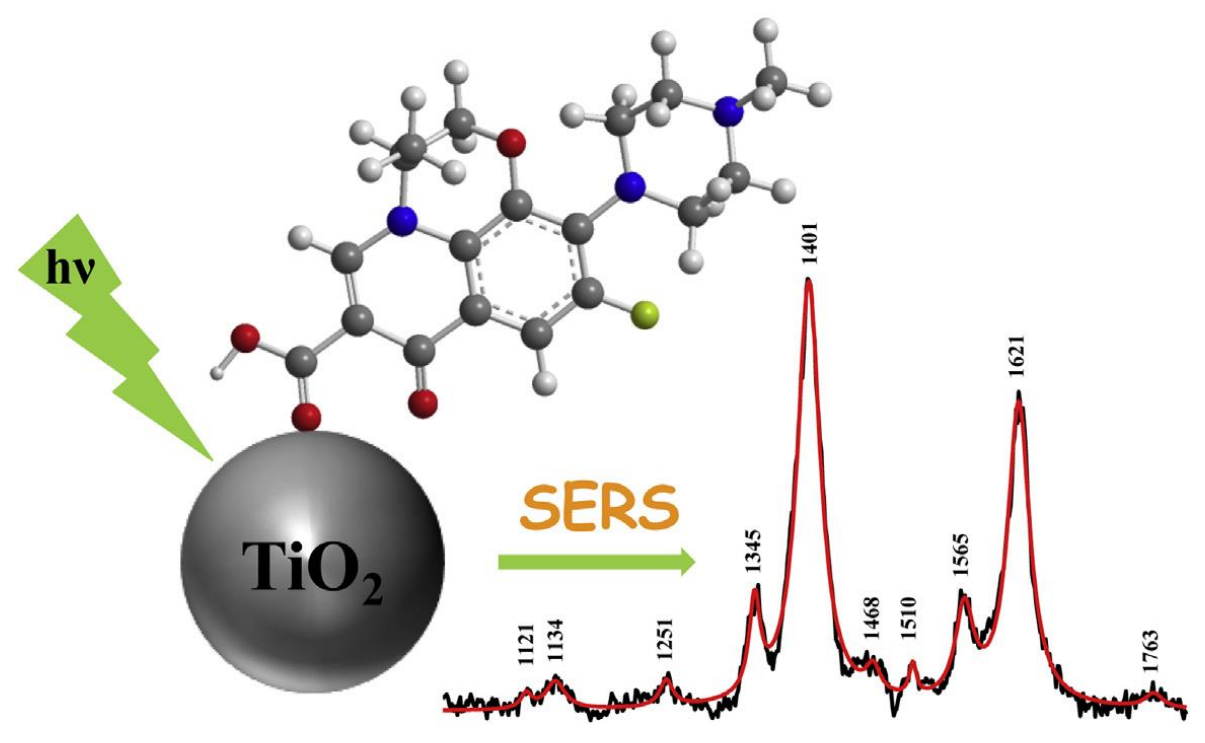

Figure 1-8: Zn-dope $\mathrm{TiO}_{2}$ nanoparticles for SERS detection of levofloxacin (Courtesy [1-43])

Additionally, Cong et.al ${ }^{[1-9]}$ have developed a non-stoichiometric $\mathrm{W}_{18} \mathrm{O}_{49}$ tungsten oxide sea urchin-like nanowire structure that is able to detect R6G dye molecules up to an enhancement factor of $\sim 10^{5}$ and with a detection limit of $10^{-7} \mathrm{M}$ concentration. Formed through hydrothermal reaction, this tungsten oxide nanostructure is able to achieve the observed enhancement of the R6G dye due to oxygen vacancy defects and charge transfer between the nanostructure and the dye molecule.

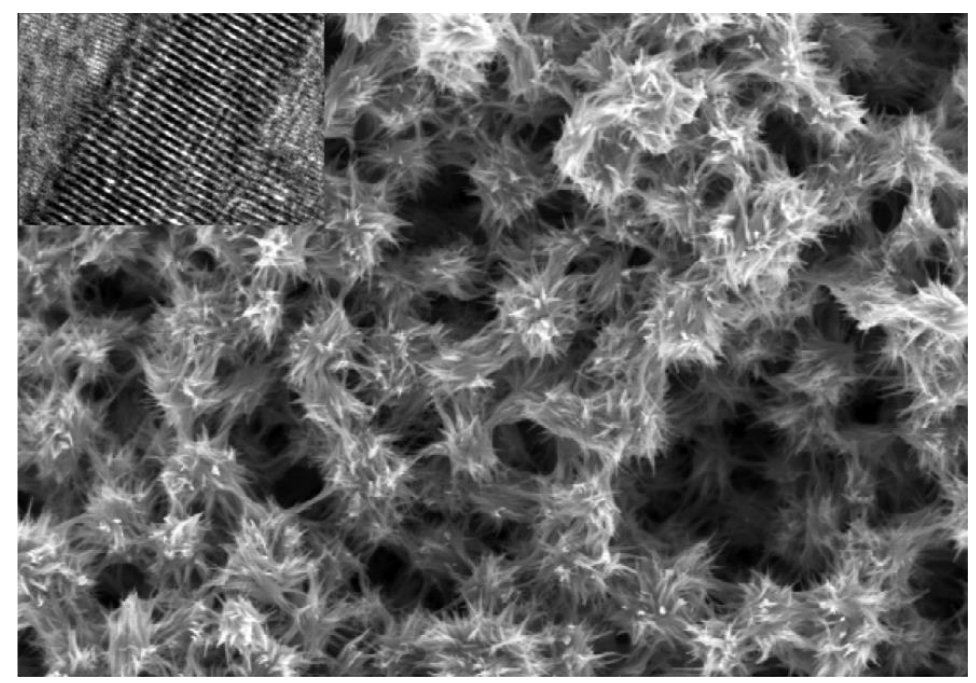

Figure 1-9: Sea urchin-like $\mathrm{W}_{18} \mathrm{O}_{49}$ nanostructures for SERS detection of $\mathrm{R} 6 \mathrm{G}$ dye (Courtesy [1-9]) 
Semiconductor nanostructures have several distinct benefits compared to noble metals that make them more advantageous as SERS-active substrates in several applications. Semiconductors are advantageous because they can be degradation resistant, have programmable surface characteristics and physical morphology, can be doped with other elements to change their electronic character, and it is possible to control their physical and chemical properties, band gap an stoichiometry; all of which are not easily controlled when fabricating NMNs. Since semiconductors do not exhibit SERS enhancement through SPR, the need for precise nanostructure size to achieve high nanogap consistency is not a necessity for semiconductor SERS substrates. However, there have been some recent studies that have shown that plasmonically active semiconductor materials can be formed through doping of free carriers and phase transitions but is still in the nascent stages of development ${ }^{[1-44]}$. In addition most semiconductor materials are considered biocompatible, which leads to their increased potential as biosensing substrates. For example Han et.al ${ }^{[1-45]}$ have fabricated $\mathrm{TiO}_{2}$ nanostructures through anodizing a Ti substrate and have been able to detect cytochrome b5, a hemoprotein present in human cell membranes.

\subsection{Nanostructure formation using ultrafast femtosecond pulsed laser ablation}

As shown previously, there are a great number of different nanostructures being developed as SERS-active substrates each being formed using a different technique; these range from gold ${ }^{[1-46]}$ or silver ${ }^{[1-47]}$ nanoparticles formed through chemical synthesis methods to a bottom-up approach to growing Si nanowires that are then coated with silver nanoparticles ${ }^{[1-48]}$ to $\mathrm{ZnO}$ nanoparticles being deposited onto carbon nanotube fibres ${ }^{[1-36]}$. Both bottom-up and top-down approach can yield highly SERS active substrates for given applications. In this thesis it is presented that by using an ultrafast femtosecond pulsed laser ionization mechanism, it is possible to active the SERS enhancing characteristics of a material, silicon, that have yet to be reported in literature. The impetus for using this nanostructure technique as a potential method to generate a new nanostructured material that has enhanced SERS properties was the works by Maznichenko et.al. ${ }^{[1-}$ ${ }^{10,1-11]}$ In these studies an ultrafast femtosecond pulsed laser ionization mechanism was employed to ionize a bulk Ti substrate to form linked multiphase $\mathrm{TiO}_{2}$ nanoparticle nanofibre network. By modifying and optimizing the control parameter of the femtosecond laser, they were able to finely control the amount of each $\mathrm{TiO}_{2}$ phase (anatase and rutile) within the nanofibres as well as the 
physical morphology of the nanofibres (nanoparticle size/ size distribution and porosity of the nanofibre network). They were able to detect CV dye and environmental contaminants, bisphenol A and diclofenac sodium salt, with a maximum observed EF of $\sim 10^{6}$. With this proven ability to create a unique nanostructure that is highly SERS-active, and the proven ability of similar Si nanostructures to be used as sensing materials by Alubaidy et.al, ${ }^{[1-49]}$ and their biocompatibility by Premnath et.al, ${ }^{[1-50]}$ demonstrate that this ultrafast laser ionization method was chosen to fabricate Si nanostructures for chemical and biosensing applications.

The aspects of ultrafast femtosecond pulsed laser ionization of silicon and how the resulting nanostructures produce SERS enhancement will be delineated in greater detail in later chapters, but a very brief description will be given here. This fabrication process involves impinging femtosecond laser pulses onto a substrate surface. These pulses will immediately begin to ionize the substrate surface causing ions to be ejected from the substrate. These ions form a plume above the substrate surface wherein the ions from the substrate and ions and molecules from the surrounding atmosphere will react and bond with each other to form ionic cluster and grow to form nanoparticles. These nanoparticles then condense and fall back to the substrate surface, build up and fuse together to form a three-dimensional nanonetwork of fibre -like nanostructures. Figure 1-10 shows a schematic of the ultrafast femtosecond pulsed laser ionization mechanism and the nanostructure formation.

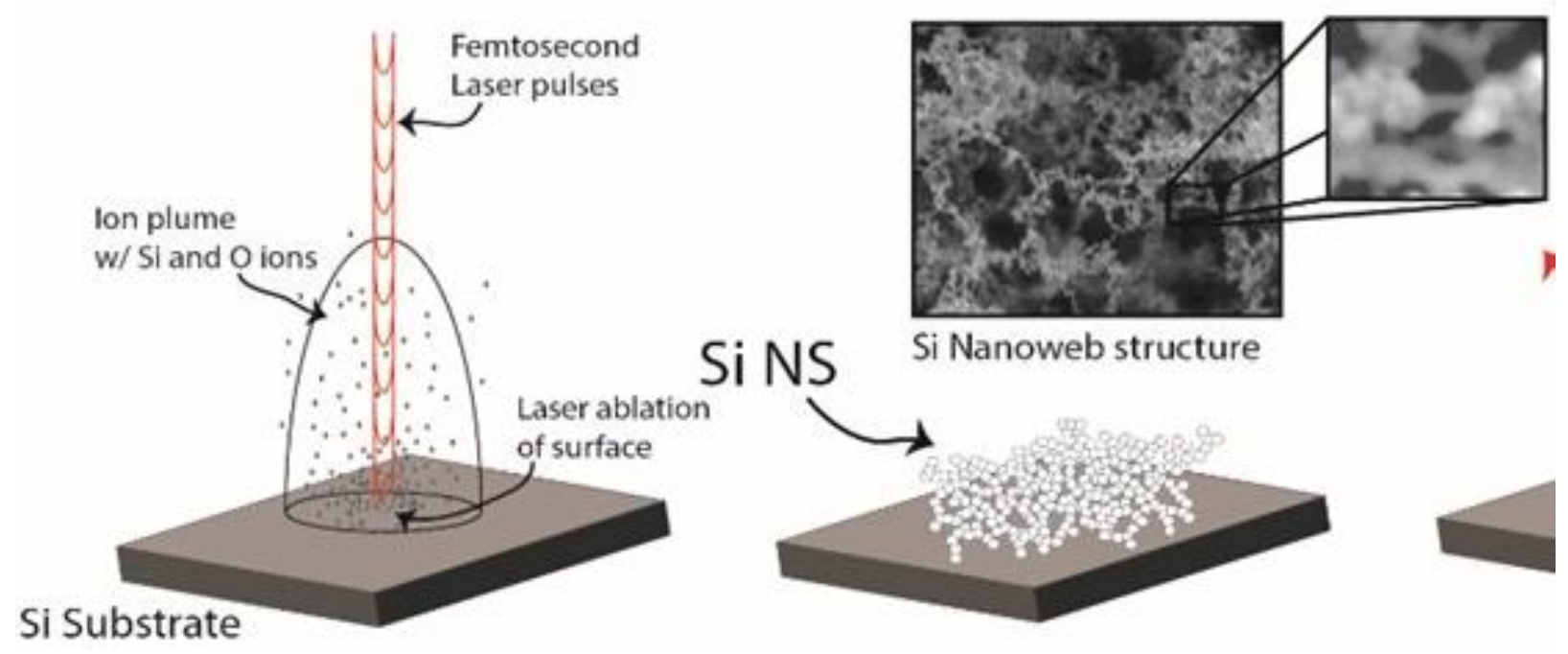

Figure 1-10: Ultrafast femtosecond pulsed laser ionisation and nanostructure formation 
Unlike nanolithography or wet chemical synthesis that make nanostructured versions of bulk materials this nanostructure generation technique fundamentally creates new materials from bulk substrates. This is because the femtosecond pulsed laser breaks down a bulk substrate into its ionic components and the ions then reform into completely distinct nanomaterials which are not possible to fabricate using conventional techniques.

\subsection{Summary}

SERS-active nanomaterials exist in a variety of forms and many different materials can exhibit SERS enhancing properties. These nanostructured materials have been shown to produce significant enhancement in detection of chemical and biomolecules alike. The disadvantages of noble metal based SERS substrates has led to the current interest in developing semiconductor nanostructures as SERS-active materials and semiconductor/metal oxide nanomaterials as SERS substrates are an emerging field of research. There is, however, a current lack in studies that are developing Si-based semiconductor SERS substrates for both chemical and biosensing applications. It will be demonstrated that a SERS-active Si nanostructure generated using an ultrafast femtosecond pulsed laser ionization mechanism can exhibit significant SERS enhancing characteristics. These Si nanostructures are able to overcome the drawbacks associated with noble metal SERS nanostructures while producing comparable SERS activity. As such these $\mathrm{Si}$ nanostructures have been established in this thesis as viable SERS chemical and biosensing platforms. 


\subsection{Research Objectives}

Si-based SERS nanostructures generated through ultrafast pulsed laser ionization offer promise as chemical and biosensing substrates. This fabrication technique is able to generate a unique nanostructure with nanoweb-like morphology as is able to be fine-tuned to modify the material chemistry of the silicon within the nanostructure. This controllability of this laser-ion fabrication technique allows for the optimization of the SERS enhancing characteristics for chemical and bioanalyte detection.

The main objective of this thesis is to generate a SERS-active Si nanostructure using the laser-ion fabrication method and to establish a Si-only nanomaterial as a viable SERS sensing platform. With the establishment of a Si nanostructure as a highly SERS active substrate, the next goal is to further enhance the SERS sensing capabilities of the Si nanostructure through additional enhancement mechanisms and modification of the nanostructure formation mechanism, to determine its viability as a biosensing platform. This will be accomplished through sub-objectives with individual tasks:

Sub-objective 1: Generate a SERS-active Si nanostructure using ultrafast femtosecond pulsed laser ionization

Task 1: Fabricate a silicon nanostructure using the ultrafast femtosecond pulsed laser technique and explore who the laser characteristics can be controlled to generate varying nanostructure formations

Task 2: Characterize the nanostructure in terms of the physical morphology and the material chemistry in order to classify how the laser-ion formation mechanism generates nanostructures

- Scanning electron microscopy (SEM) for overall nanostructural morphology determination, X-ray diffraction crystallography (XRD) to determine the crystallographic nature and composition of the nanostructure, transmission electron microscopy (TEM) for nanospheroid size and size distribution analysis, and nanospheroid morphology analysis

Task 3: Determine the Raman characteristics of the fabricated nanostructures through nano-Raman spectral analysis of the Si nanostructure 
Task 4: Study and evaluate the SERS enhancing properties of the Si nanostructure by the spectral response to chemical dyes rhodamine $6 \mathrm{G}(\mathrm{R} 6 \mathrm{G})$ and crystal violet $(\mathrm{CV})$

Sub-objective 2: Determine the SERS enhancing characteristics of a semiconducting $\mathrm{Si}$ nanostructure with additional noble metal nanostructures for chemical sensing and potential biosensing applications

Task 1: Fabrication of the silicon nanoweb structure using ultrafast pulsed laser-ion technique and subsequent ornamentation of gold nanospheres on nanoweb surface at varying time scales

Task 2: Characterize the Au ornamented nanoweb structures in terms of physical morphology and material chemistry using TEM imaging, x-ray photoelectron spectroscopy (XPS) and Raman spectroscopy.

Task 3: Determination of the effects of Au nanosphere concentration on the Raman enhancement of the nanostructures compared to bare Si nanostructures. An evaluation of the SERS enhancement produced by the Au ornamented nanoweb of chemical dye $\mathrm{CV}$ and bio-label molecule R6G

Sub-objective 3: Determine the function of a dual mechanism SERS enhancement with a Si nanostructure and an $\mathrm{Au}-\mathrm{Au} / \mathrm{Pd}$ boosting source for biosensing applications

Task 1: Synthesis of a hybrid Si nanostructure using the ultrafast pulsed laser ionization technique and physical deposition of $\mathrm{Au}$ and $\mathrm{Au} / \mathrm{Pd}$ nanospheres on Si nanostructure surface.

Task 2: Material characterization of the nanostructures using TEM imaging, XRD, and Raman spectral analysis

Task 3: Evaluation of the Raman spectra produced by the Au and Au/Pd coated Si nanostructures relative to the bare Si nanostructures to determine primary and secondary boosting potential. Application of a protein biomolecule L-glutathione (GSH) onto these nanostructure surfaces to determine potential as SERS biosensing platform and the boosting efficiency of the $\mathrm{Au}$ and $\mathrm{Au} / \mathrm{Pd}$ nanospheres

Sub-objective 4: Evaluate the role of introducing quantum-scale defects into the Si nanospheroid has on the SERS-based biomolecular diseases signalling.

Task 1: Induction of quantum scale defects into Si nanospheroids by amendmentation of ioninteractions during ultrafast femtosecond laser ionization 
Task 2: Characterization of the resultant nanostructures using TEM/HRTEM image analysis, XRD and Raman spectral analysis

Task 3: Development of a theoretical understanding of how the quantum-scale defects within the resulting Si nanostructures cause Raman activation and SERS detection of biomolecule analytes Task 4: Determine potential of Si nanostructures with quantum scale defects as an early s-tage universal biomolecular SERS nanoprobe by high sensitivity detection of disease signalling analytes.

\subsection{Organisation of the Dissertation}

Chapter 2 describes the synthesis of Si nanowebs structures using the ultrafast femtosecond pulsed laser ionization mechanism and how these hybrid Si nanostructures exhibit SERS enhancing characteristics

Chapter 3 describes the use of SPR through Au nanoparticle ornamentation to add an additional layer of SERS enhancement to the hybrid Si nanostructures and how this $\mathrm{Au}$ ornamentation process can be optimized to maximize the additional enhancement to the detection of chemical and bio-label molecules.

Chapter 4 describes how $\mathrm{Au}$ and $\mathrm{Au} / \mathrm{Pd}$ nanospheres on the surface of the hybrid Si nanoweb structure give a secondary SERS boosting source to the primary Si nanoweb boosting source for the detection of a protein biomolecule L-glutathione

Chapter 5 delineates the generation of quantum-scale defects within the Si nanostructures by ioninteraction amendmentation and the effects these defects have on the detection of disease signalling biomolecules.

Chapter 6 summarizes the results of these studies, and proposals for the future direction of this research. 


\subsection{Candidate Contribution}

This thesis dissertation presents work performed by the candidate and supervisors in the form of published journal articles, journal articles under peer review and manuscripts pending review for submission to peer-reviewed journals. The contribution form the candidate for each of these studies was to perform sample preparation, obtain experimental results, graph and evaluate experimental data, illustrate conceptual diagrams and schematics, and write and edit manuscripts for submission to peer reviewed journal. The contributions from the candidates supervisors was to advise the candidate on the concepts and design of the experimental studies, aid in interpreting results and, to revise and comment on written work. As such, the candidate is the primary author for all published works, submitted works and works pending submission. 


\section{Chapter 2}

\section{Programmable SERS active substrates for chemical and biosensing applications using amorphous/crystalline hybrid silicon nanomaterial}

\section{Published in Nature Scientific Reports, January 2016}

SERS sensing techniques offer a non-destructive and highly sensitive method for detecting chemical and biomolecules for various applications. However, the majority of SERS sensing nanomaterials rely solely on noble metal nanostructures as the primary SERS enhancement source; noble metal nanostructures require highly precise feature size and distribution to obtain significant and reliable SERS enhancement. Recent advances have shown that semiconductor nanostructures have the potential to exhibit SERS enhancing characteristics on par or greater than similarly fabricated noble metal nanostructures. In this chapter, the creation of a SERS-active hybrid Si nanoweb structures using an ultrafast femtosecond pulsed laser ionization technique. By using the flexibility that this technique allows in terms of modifying the properties of the pulsed laser, the generated Si nanoweb structure can have highly programmable physical morphology and material chemistry. These physical and material properties govern how this nanostructure is able to produce for the first time SERS enhancement of chemical analytes using only a Si nanostructured material.

\subsection{Introduction}

The field of nano-Raman sensing is growing year by year with further advancements to enhancement of well-established sensing materials. ${ }^{[2-1,2-2]}$ Raman scattering is an important technique in the field of chemical and bio-sensing because it offers the ability to detect these analytes at single molecule concentrations. ${ }^{[2-3]}$ Nano-Raman techniques also have the ability to detect a wide breadth of analytes; not only can nano-Raman device detect various chemicals including, pollutants in water supplies, ${ }^{[2-4]}$ explosive chemicals, ${ }^{[2-5]}$ and for pharmaceutical chemical identification, ${ }^{[2-6]}$ nano-Raman has the ability to be used a sensing technique for many 
biomolecules as well. Nano-Raman sensors also have the capability to detect various cancer cells, ${ }^{[2-7,2-8]}$ bacteria, ${ }^{[2-9]}$ RNA and DNA, ${ }^{[2-10,2-11]}$ and viruses. ${ }^{[2-12]}$

The primary issue nano-Raman scattering observation is the intensity of the scattered signal; only $10^{-12}$ incident photons ${ }^{[2-13]}$ will inelastically scatter resulting in Raman characterization being an impractical tool without significant enhancement in signal. SERS enhancement serves as a main focus for current research as nano-Raman sensing due to the extremely high enhancement factor and the exceptional sensitivity that SERS activation can achieve. It has been established that the main source of SERS enhancement for metallic nanomaterials comes from a phenomenon known as surface plasmon resonance (SPR); which is the collective oscillation of electrons about atomic nuclei caused by incident electromagnetic radiation. The increase in sensitivity to an analyte originates from the enhancement of electric field caused by SPR which is transferred to an analyte molecule resulting in a larger cross-section of Raman scattered photons. ${ }^{[2-14]}$ Noble metals (gold, silver, etc.) have been predominantly used as SERS materials since they have a well-established property of exhibiting surface plasmon resonance (SPR) in the visible and near-infrared (NIR) spectrum, the wavelength range for most Raman lasers ${ }^{[2-15]}$. While SPR is the major source of Raman enhancement, it alone cannot provide the tremendous observed enhancement factors of these nanostructured materials; the physical morphology plays an important role as well and works in concert with SPR. Many different nanostructures have been created and have demonstrated enhanced Raman scattering, including nanostars, ${ }^{[2-16]}$ nanofilms on arranged arrays of nanowires, ${ }^{[2-17]}$ hollow or solid nanocubes ${ }^{[2-18]}$ or nanoshells. ${ }^{[2-19]}$ The common origin of enhancement for most of these nanostructures is the creation of localized regions of concentrated electromagnetic field, which are known as Raman or plasmonic hotspots. Hot spots usually form in the nanogaps between two nanostructures ${ }^{[2-20]}$, the simplest being the gap between two nanoparticles ${ }^{[15]}$ but can be formed between nanostructures of many types; ${ }^{[2-21]}$ this means however, that the creation of nanogaps is highly dependent on the nanostructure size, the wavelength of Raman laser and the analyte molecule size, ${ }^{[2-13]}$ but with precise control single molecule detection is possible. ${ }^{[2-3]}$

There is however, a significant lack of research focus on developing materials that utilize semiconductor based nanomaterials, specifically silicon, for use as SERS enhancement substrates. This is due to nanostructured silicon materials being relatively dormant in terms of direct SERS activation when fabricated using conventional synthesis techniques. For example Wells et.al have 
achieved an EF value of 510 for the detection of $\mathrm{Zn}$ phthalocyanine on a Si nanopillar array fabricated using nanolithography techniques ${ }^{[2-22]}$. Other researchers have also reported EF values 8-28 ${ }^{[2-23]}, 7.1-70^{[2-24]}$, and $10^{3[2-25]}$. While substantial achievements in Si-based SERS activation, these EF values are orders of magnitude lower than the EF values reported from noble metal nanostructures; for example Garcia-Leis et.al ${ }^{[2-26]}$ have reported an EF value of $1.72 \times 10^{5}$ for $\mathrm{Ag}$ nanostars, Wang et.al ${ }^{[2-27]}$ have reported a maximum EF value of $10^{7}$ for gold nanoparticle arrays and Tao et.al ${ }^{[2-28]}$ have reported an EF value of $2 \times 10^{9}$ for Ag nanowire monolayers. As such, of the currently researched SERS nanomaterials, nanostructured silicon is employed primarily as a scaffold or substrate for noble metal Raman active nanostructures ${ }^{[2-29-2-31]}$; nanostructured silicon does not play a major active role in Raman enhancement. There is a significant incentive to fill this void of SERS active silicon nanomaterials because silicon is the building block material for all existing electronic devices and having access to a Raman active nanostructured silicon material would not require the development of new integration techniques for creating Raman sensing devices. A sizable amount of research has been conducted to investigate the Raman activity of semiconductor nanomaterials yielding promising results. SERS enhancement has been observed in $\mathrm{ZnO}^{[2-32]}, \mathrm{ZnS}^{[2-33]}, \mathrm{CdS}^{[2-34]}$ and $\mathrm{CuO}^{[2-35]}$, each in colloidal suspension. Competitive $\mathrm{EF}$ values, on the order of $10^{6}$ have also been observed in three dimensional $\mathrm{TiO}_{2}$ nanostructures. ${ }^{[2-36,2-39-2-}$ ${ }^{46]}$ These semiconductor materials and to a greater extent silicon, are more attractive as SERS materials due to the breadth of controllability over material properties (including band gap, dopants, physical morphology, stoichiometry, phase crystallinity, nanostructure size distributions etc. ${ }^{[2-40]}$ ) that semiconductors have over metals. Therefore a highly SERS active Si nanomaterial opens up new opportunities to use as a SERS sensing substrate rather than cost-prohibitive, difficult to fabricate noble metal SERS nanostructures that lack depth of control over material properties and physical morphology. The use of Si based SERS nanomaterials could lead to the proliferation of economical SERS detection devices for a vast array of applications.

With this current research, a new phenomenon for SERS activation using nanostructured Si that can be precisely controlled with the formation mechanism that allows for the creation a SERS active material from a dormant Raman Si wafer material. In this study, an entirely new type of nanomaterial has been crated which is as to yet, not producible by other methods; the creation of hybrid crystalline/amorphous Si nanospheroids within a laser ionization plume, which fuse and deposit onto the silicon substrate as an interconnected nanoweb network. Rather than individual 
crystalline and amorphous nanoparticles, with the ultrafast femtosecond laser synthesis it is possible to create nanospheroids that have regimes of crystalline and amorphous silicon throughout individual nanospheroids. By altering the ionization mechanisms and temperature within the laser plume, the structural arrangement of the silicon ions to form this hybrid silicon nanomaterial can be precisely controlled. Not only can the crystalline/amorphous content of the individual nanospheroids be programmed, the morphology of the nanospheroids and how they will arrange themselves when deposited on the substrate surface can be manipulated. Due to this unique particle formation mechanism, these formed nanospheroids are distinctive in their structural character which allows them to be much more SERS active than bulk single crystal Si due to a higher concentration of grain boundaries within the nanospheroids. This combination of amorphous/crystalline grains within individual nanospheroids of an interconnected nanospheroid network is an entirely new concept for nano-Raman activation. Figure 2-1 is an overall schematic and SERS enhancement figures for molecular dyes on Si nanoweb structures. 

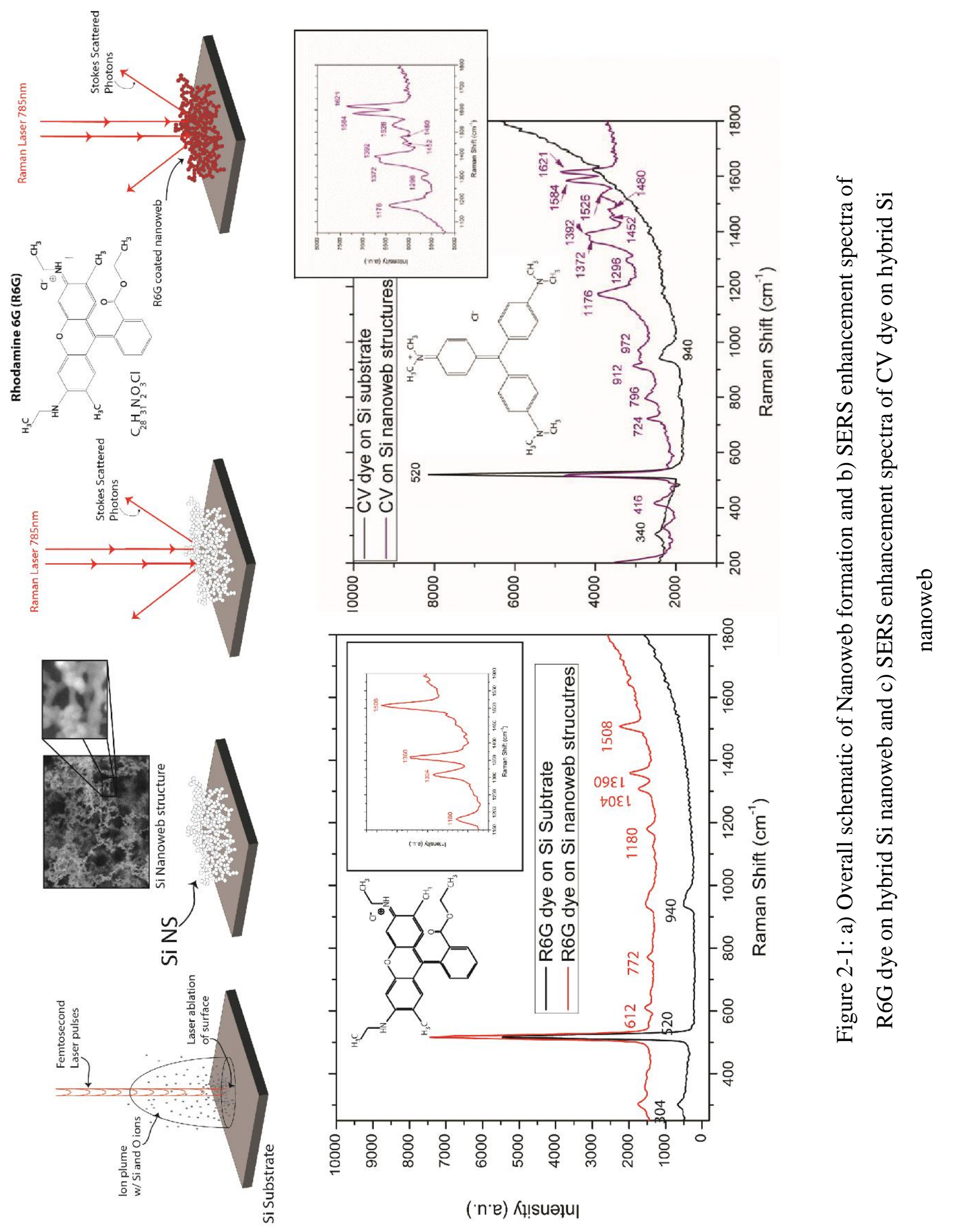
The goal with this research is to demonstrate the ability to synthesize an entirely new silicon nanomaterial has SERS enhancing properties not present in bulk silicon material or other nanostructured Si materials and that precision control of the production of this unique nanomaterial can be achieved, which is unattainable with other nanomaterial formation mechanisms. In addition, a demonstration of the viability of a Si Raman active nanomaterial as a chemical sensing substrate without the need for traditional noble metal SERS activation is observed.

\subsection{Experimental Method}

\subsubsection{Laser Ablation/Raman analysis/Material characterization}

A pulsed Yb-doped fibre amplified femtosecond laser was used to fabricate the silicon nanostructures. In order to maximize the control over the nanoweb fabrication, some of the parameters of the laser need to be fixed, in this experiment the laser wavelength (1030nm), polarization (circular) and the laser power $(16 \mathrm{~W})$ were fixed. The parameters that were varied were the repetition rate, dwell time and pulse width.

The nanostructures were created on a $5 \times 5 \mathrm{~mm}$ array of points with a $50 \mu \mathrm{m}$ point spacing; the array was plotted using EzCAD software and controlled using a piezo-driven raster scanner. The silicon substrates that were used were crystalline silicon wafers with orientation $<110\rangle$.

Each ablation area was examined before and after a dye was coated onto the ablation using a B\&W Tek, Inc NanoRam ${ }^{\circledR}$ handheld Raman system. The Raman excitation laser used has a wavelength $785 \mathrm{~nm}$ at a power of $350 \mathrm{~mW}$. The bare nanofibre ablation areas were analyzed using the Raman laser to determine how the nanostructures change the Raman spectra compared to the unablated substrate in terms of the Raman intensity as well as any structural/compositional changes caused by the ablation. The dyes used to test the SERS enhancement factor of the silicon nanostructures were Rhodamine 6G and Crystal Violet, which are popular dyes for SERS analysis due to their large Raman cross-section. To determine a sensitivity range for the Si nanoweb structures, each dye was coated onto individual ablation areas at two different concentrations, $8 \times 10^{-3} \mathrm{M}$ and $8 \times 10^{-}$ ${ }^{6} \mathrm{M}$. A single drop of each dye concentration for both dyes was applied to a separate ablation area prior to Raman analysis. Each resultant Raman spectra were obtain at 3s collection time and repeated in triplicate then averaged. 
To confirm the presence of and characterize the structure of the silicon nanoweb networks, highresolution scanning electron microscopy (HRSEM) was used. SEM was used to determine the nanospheroid size distribution and nanogap size distribution for each ablation area. Using the HRSEM images of the nanoweb substrates and ImageJ image processing software, the average size of the nanospheroid was calculated. Using ImageJ, the particle size is calculated manually; first the scale is adjusted based on the magnification of the image then the spheroid size is measured from the outer diameter of each spheroid; the nanogap size distribution was measured using the same technique. To obtain cross-sectional images, substrates were split down the middle through an ablation area, and the nanofibres were imaged at an angle. Gold sputtering of the nanoweb was necessary because of excessive charging due to the fibres being composed of silicon.

High-resolution transmission electron microscopy (HRTEM) was also used to image and analyze the nanoweb and nanospheroid shape and size. HRTEM involves using a carbon grid to swab the sample to attach nanospheroid clusters to the grid and then the grid is scanned. Using the fast Fourier transform (FFT) analysis the crystal orientations present within each nanospheroids was determined.

X-ray diffraction was used to analyze the composition of the nanostructures as well as the relative proportion of crystalline and amorphous content of the nanostructures. The XRD data was collected using a Bruker AXS D8 Advance microdiffraction system equipped with a $\mathrm{Cu}-\mathrm{K}$ source and graphite monochromator to eliminate unwanted $\mathrm{Cu}-\mathrm{K}$-beta lines. In order to obtain the relative proportion of crystalline to amorphous content, the XRD data underwent Reitveld analysis.

\subsection{Results and Discussion}

\subsubsection{Hybrid Nanospheroid formation}

The unique nanomaterial created with the ultrafast femtosecond laser is a material that, according to literature, cannot be formed using any other fabrication technique; lasers with longer pulse widths (nanosecond, picosecond, etc.) are unable to create this material because only a laser with femtosecond pulse width is able to cause such high temperatures that when the pulses strike the silicon surface, Si atoms are immediately ionized and form an ion plume above the silicon surface without the loss of energy to heating of the substrate. ${ }^{[2-41]}$ Figure 2-2 is schematic showing the laser ion plume formation mechanism with SEM images of formed nanoweb structures. The 
observed nanomaterial is an interconnected network of hybrid amorphous/crystalline nanospheroids to form a nanoweb structure. Figure 2-3 shows the nanoweb structure and the interconnected nanospheroid nature of the nanoweb material.
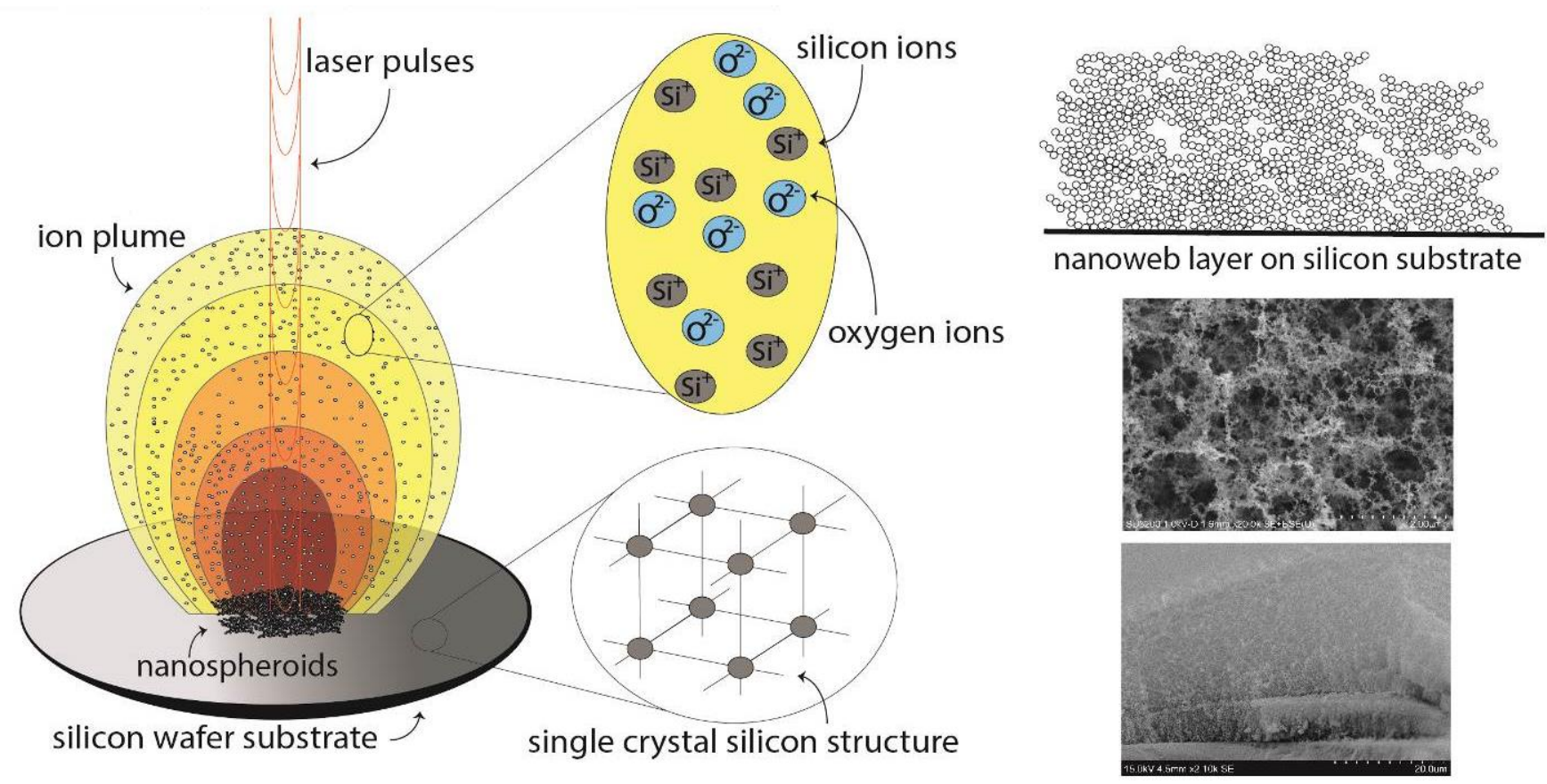

Figure 2-2: a) Schematic representation of ion plume nanospheroid formation mechanism, b) representation of nanoweb layer on substrate surface, c) SEM images of nanoweb layer 


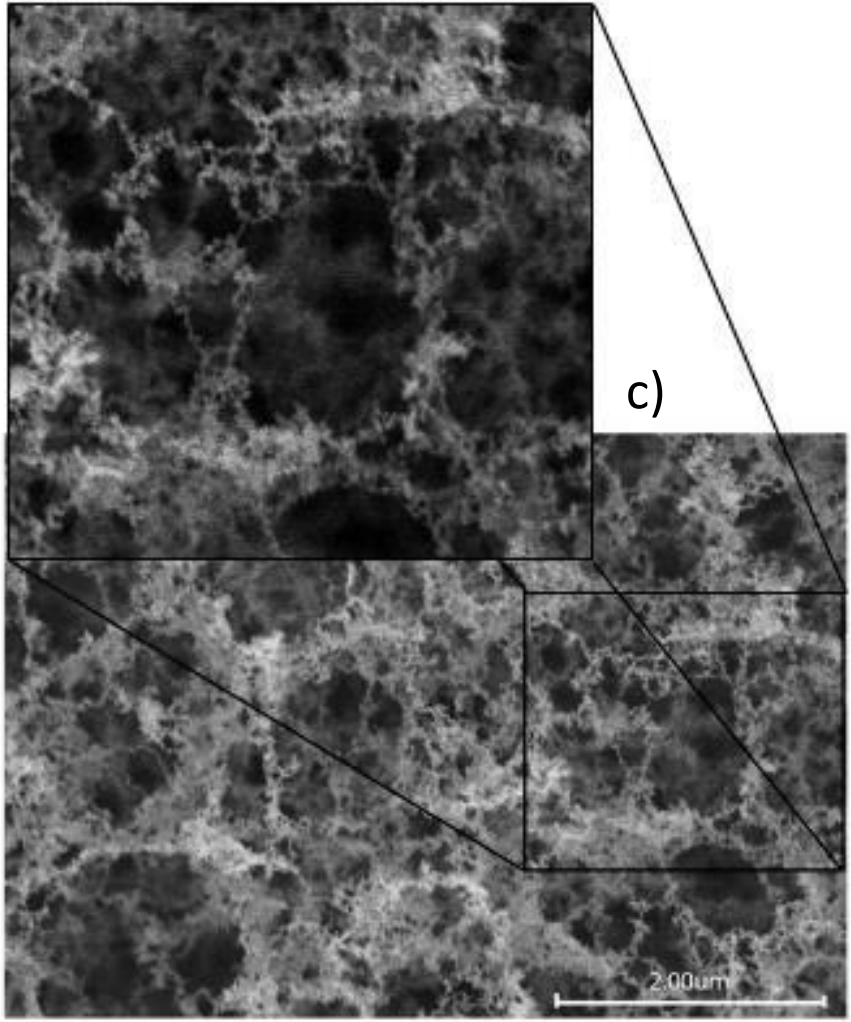

a)

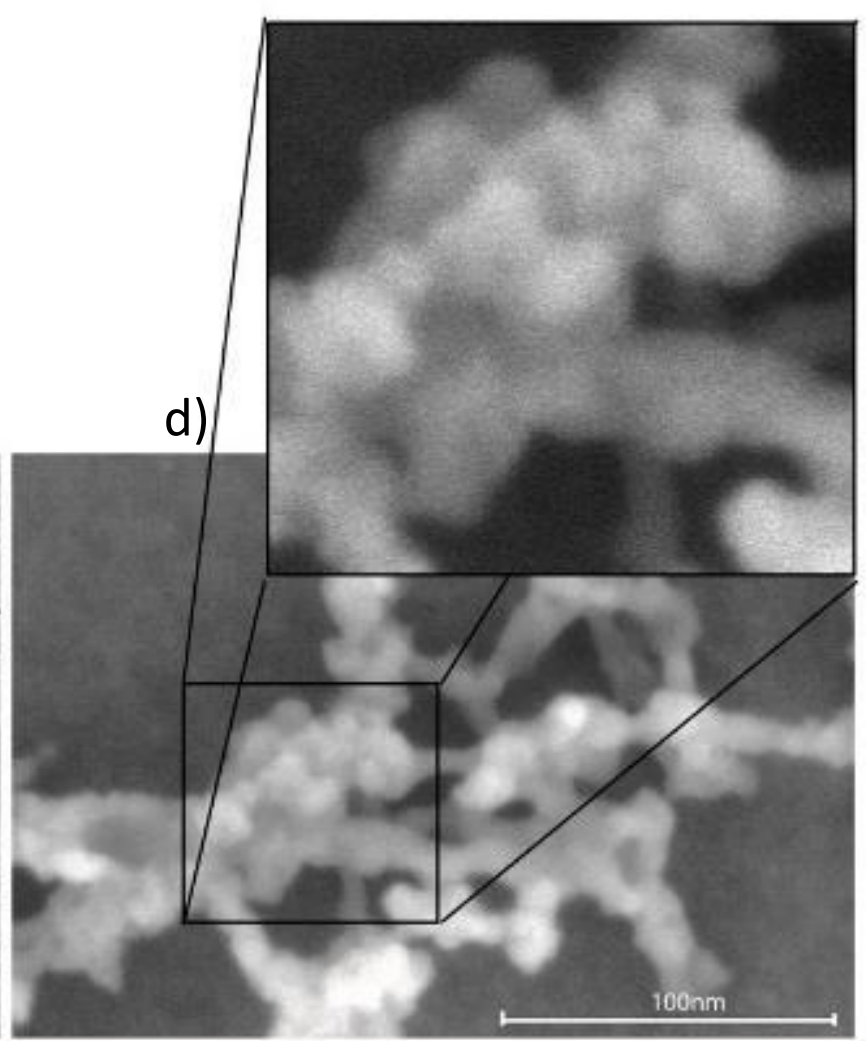

b)

Figure 2-3: HRSEM images of hybrid amorphous/crystalline silicon nanoweb structures showing a) 3D nanoweb morphology and b) individual fused nanospheroids with zoomed images c), d) to show detail

It is hypothesized that this hybrid of crystalline and amorphous phases within individual nanospheroids is due to the rapid fluctuation of temperature of the plume caused by the laser pulses. The plume temperature is quickly shifting creating moments of higher temperature and relatively lower temperature. ${ }^{[2-42]}$ At higher temperatures, the Si ions when interacting are more likely to arrange themselves into amorphous structures because they will have higher thermal energy and as such will behave in a less organized fashion, ${ }^{[2-43]}$ resulting in a more random assembly. Between pulses however, the plume temperature will be lower ${ }^{[2-42]}$ because the laser is not providing additional thermal energy, therefore the Si ions will be less energetic and assemble themselves into a more organized and lower energy arrangement, resulting in a crystalline structure. As time passes, these Si atoms that have formed amorphous or crystalline arrangements will collide and bind together to form random arrangements of amorphous and crystalline nanograins. These will then coalesce and deposit themselves onto the substrate surface as 
nanospheroids that have regions, within the same spheroid, of amorphous and crystalline silicon. Figure 2-4 is a schematic diagram of this process.
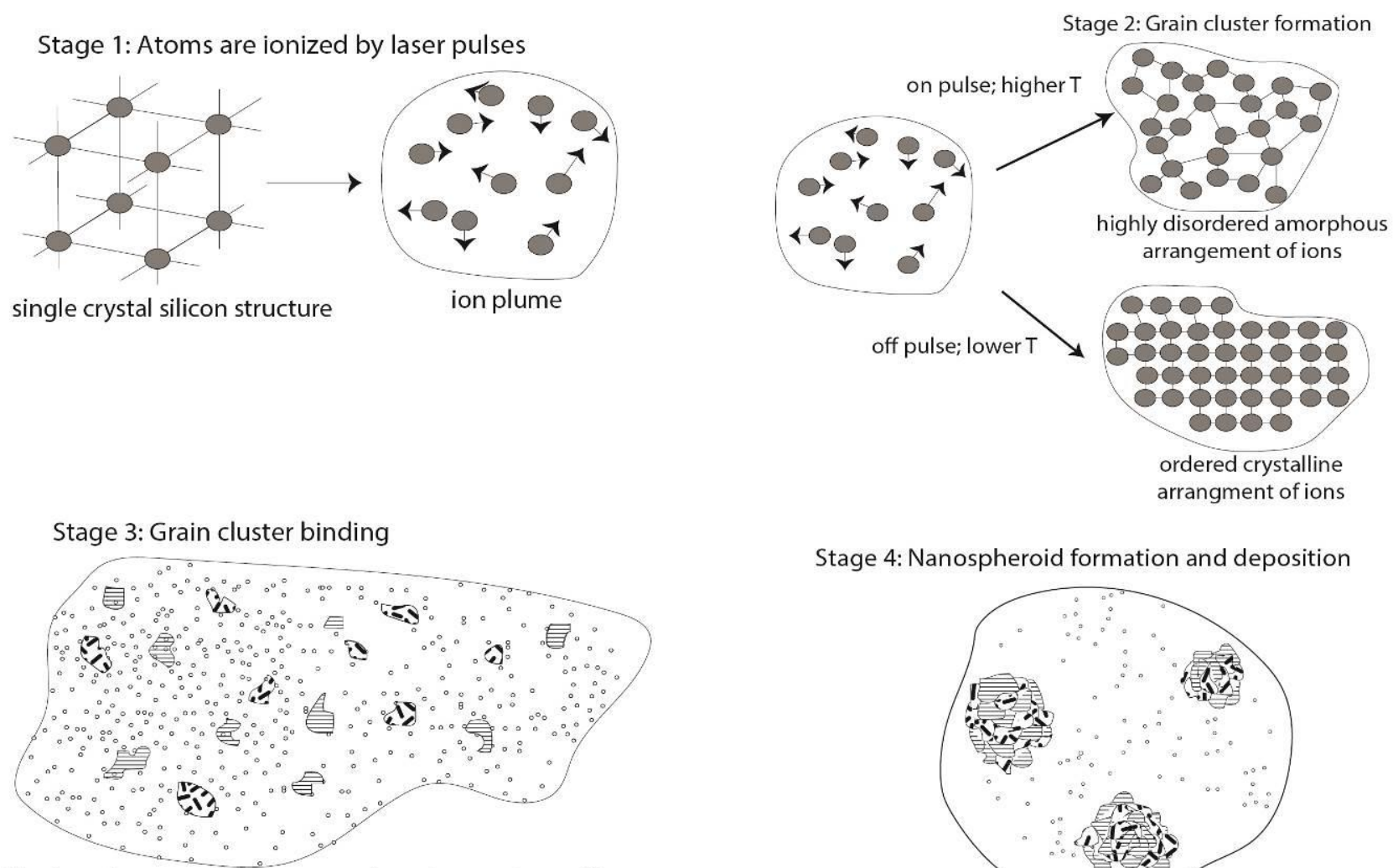

The ion plume contains nanograins of amorphous $\mathrm{Si}$ and crystalline Si. Nanograins cluster together to form multi-grained amorphous/crystalline nanoclusters.

\footnotetext{
要crystalline

厎amorphous
}

Stage 4: Nanospheroid formation and deposition

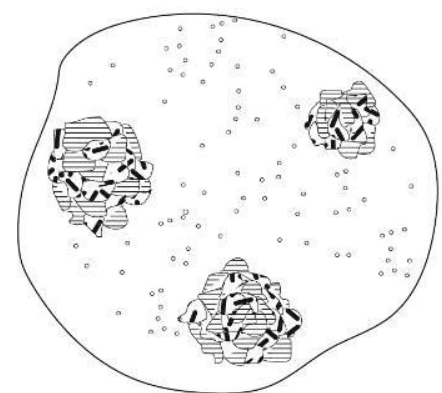

As the plume temperature decreases below the threshold temperature, particle growth is quenched. Nanospheroids fall to the substrate still at relativelty high temperature which allows them to bond and form web-like strucutres

Figure 2-4: Schematic representation of theoretical hybrid amorphous/crystalline nanospheroid

\section{formation mechanism}

The XRD results (Figure 2-5) show that within the nanoweb samples the observation of the presence of both crystalline and amorphous $\mathrm{Si}$ and amorphous $\mathrm{SiO}_{2}$. These spectra also show three sharp peaks indicating the presence of multiple orientations of crystalline Si. There is a sharp peak for single crystal Si from the [111] plane for each sample and sharp peaks for the [220] and [311] planes. The existence of these multiple orientations of crystalline Si supports the hypothesis that nanospheroids with randomly oriented grains of Si are being created. These peaks are present for 
each XRD spectra in Figure 2-5; therefore regardless of plume conditions nanospheroids with randomly oriented Si grains are generated.

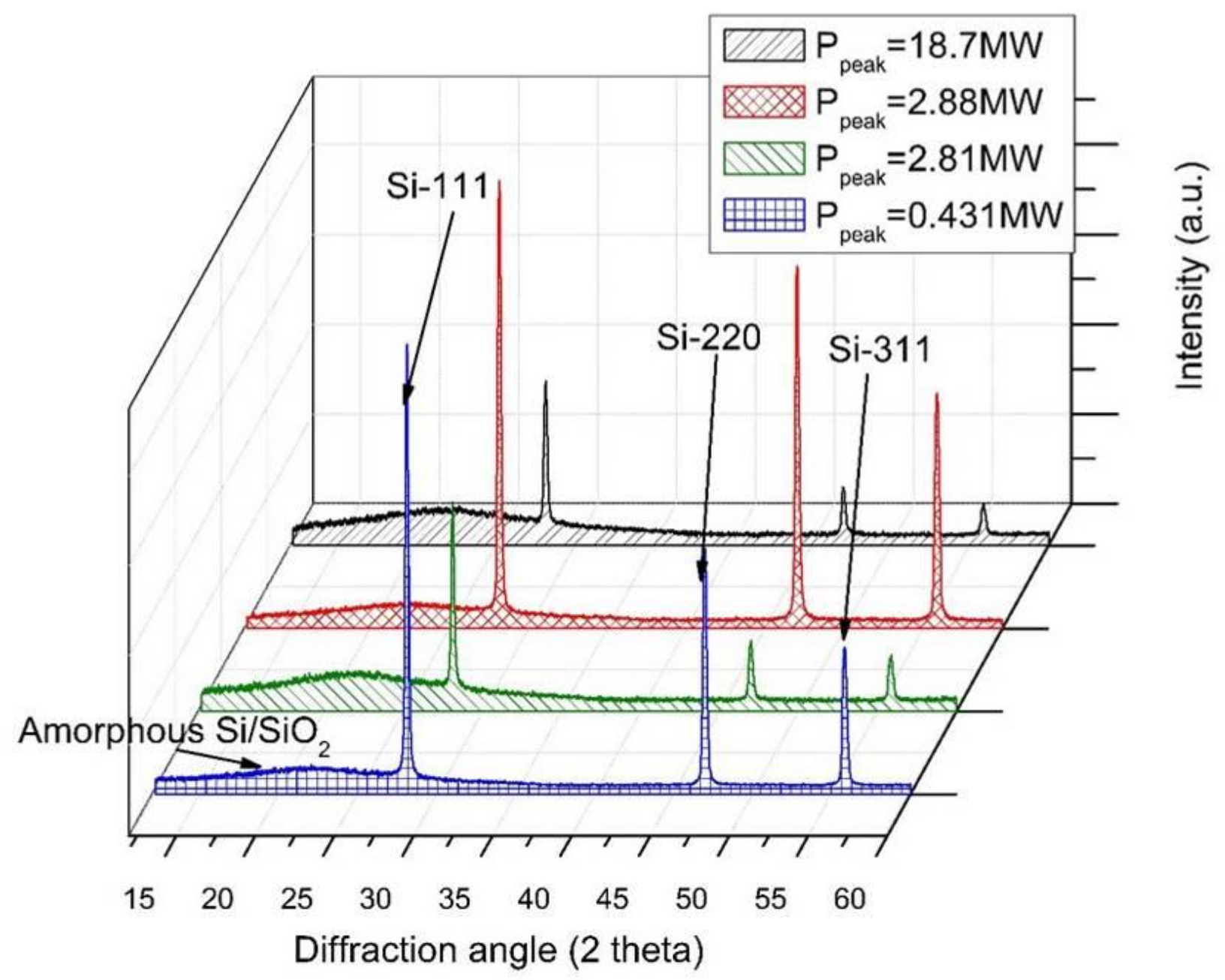

Figure 2-5: XRD Spectra for nanoweb structures

The other major observation from the XRD spectra is that each nanoweb layer has a significant portion of amorphous content, from which it can be surmised that in addition to the multiple crystallographic orientations of $\mathrm{Si}$ present in each particle, amorphization of silicon is occurring during the particle formation process as well as an oxidation process which forms amorphous $\mathrm{SiO}_{2}$. ${ }^{[2-43]}$ This coincides with the proposed mechanism, in which the individual nanospheroids consist of Si grains with different crystallographic orientations and amorphous Si grains.

Using Reitveld analysis the proportion of crystalline to amorphous material is present within the nanospheroids is determined (Figure 2-6). Reitveld analysis spectra for $214 \mathrm{fs}$ at $4 \mathrm{MHz}$ are not 
included due to equivalent sample compositions between $214 \mathrm{fs}$ and $1428 \mathrm{fs}$ at $4 \mathrm{MHz}$. The sample spectra for $1478 \mathrm{fs}$ at $26 \mathrm{MHz}$ are not included for the same reason. The Reitveld analysis shows that at $214 \mathrm{fs}$ the sample is $76 \%$ amorphous and $24 \%$ crystalline and at $1428 \mathrm{fs}$ the sample is $40 \%$ amorphous and $60 \%$ crystalline.

a)
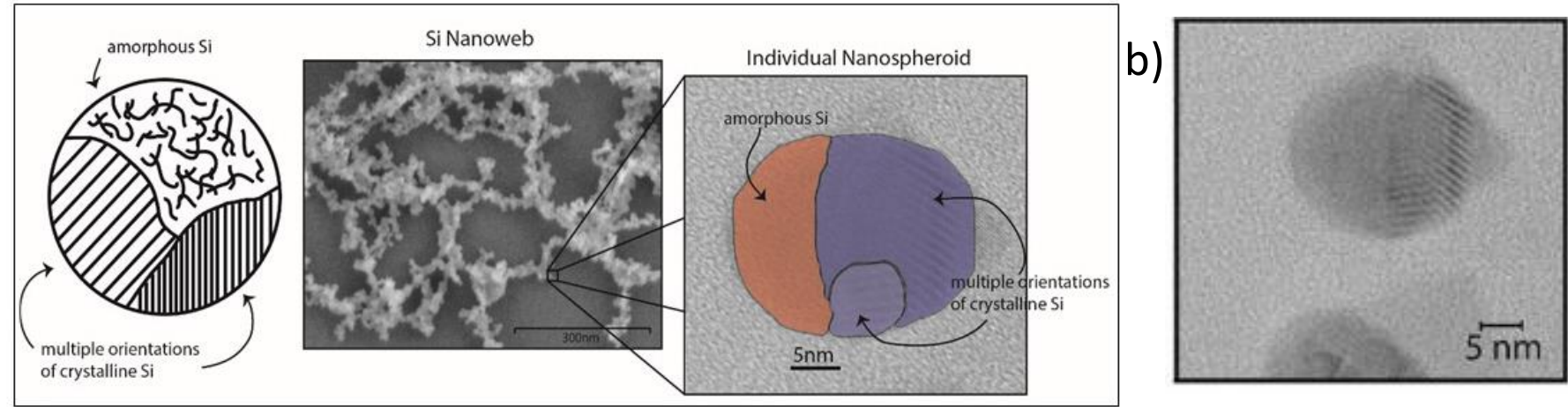

c)

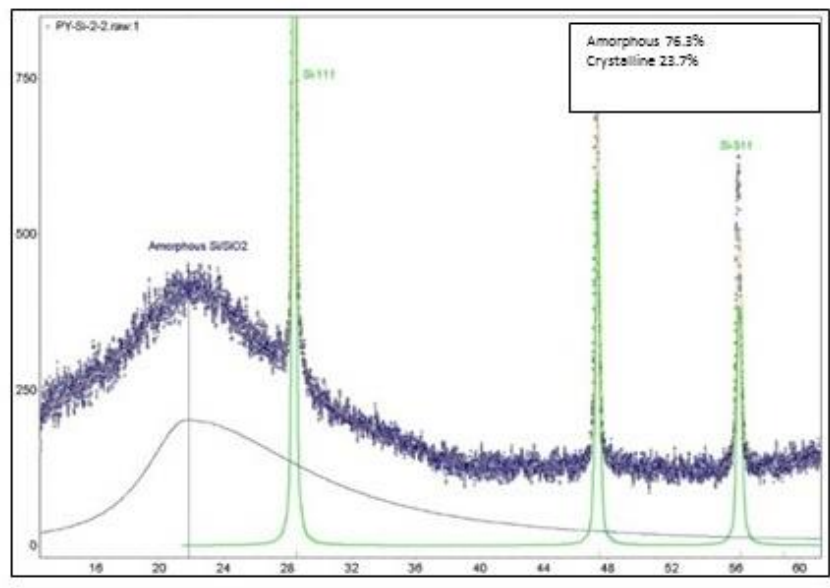

d)

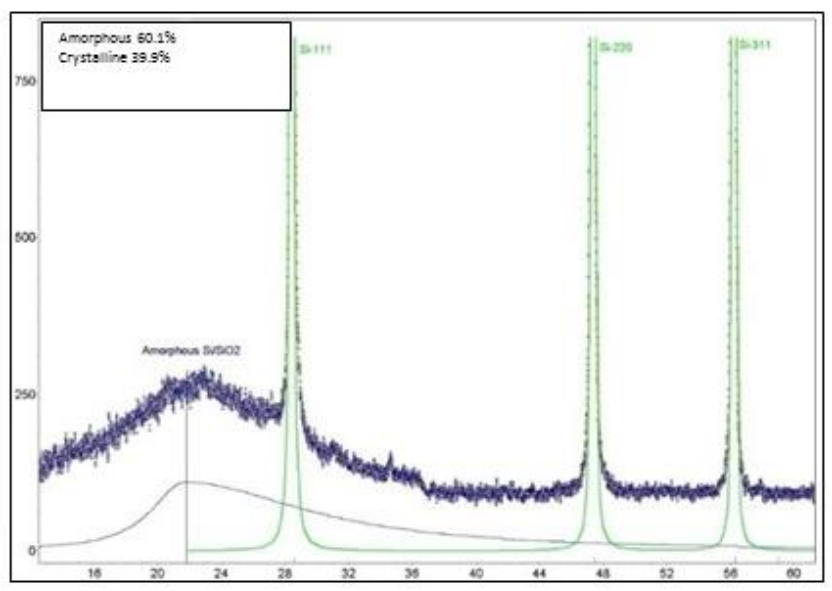

Figure 2-6: a) Schematic showing hybrid nature of nanospheroids with HRSEM and HRTEM images showing nanoweb structure and b) amorphous/crystalline grains within a nanospheroid and Rietveld spectra for nanoweb created at c) high peak power and d) lower peak power With the level of control that over the conditions within the Si ion plume, it is possible to influence the particle formation mechanism in order to construct a selected physical morphology of the deposited nanoweb layer. The two aspects of the physical morphology that are highlighted in this research are the diameter of the deposited hybrid nanospheroids and the thickness of the nanoweb layer on the substrate surface. To design the morphology of the nanoweb layer a modification of the laser interactions with the substrate surface is necessary; control over the time interval of each laser pulse, known as the pulse width, the time between the laser pulses, known as the repetition 
rate and the time spent ablating each ablation point known as the dwell time each contribute to observed changes in the nanoweb morphology.

As with the hybrid structure formation, controlling the morphology of the individual nanospheroids, specifically the diameter of the nanospheroids, is dependent on the temperature within the ion plume. At high plume temperatures, enough energy is supplied to the nanograins so that they can continue to grow and bind with other nanograins, ${ }^{[2-44]}$ and thus increase in size. However, when the temperature of the plume decreases below a certain point the growth rate of the grains is inhibited, effectively quenching grain growth ${ }^{[2-41]}$ and initiates the nanospheroids formation stage and defining the particle size. Since the laser attributes define the plume temperature, direct control over the diameter of the nanospheroids is observed.

This change in particle size is due to the effects of the repetition rate and the pulse width on the temperature within the ion plume in which the particles are forged. By changing the repetition rate and the pulse width, the number of pulses that strike the sample surface every second and the time between laser pulses striking the substrate are programmed respectively. Both of these laser parameters will affect the peak power of the laser pulses and thus the energy of the pulses. Peak power and pulse energy are calculated using the following two equations:

$$
\begin{array}{ll}
P_{\text {peak }}=\frac{P_{\text {avg }}}{t_{p} f_{p}} & \text { (Equation 2-1) } \\
E_{p}=P_{\text {peak }} t_{p} & \text { (Equation 2-2) }
\end{array}
$$

Where $P_{\text {peak }}$ is the peak power $P_{a v g}$ is the average power, $t_{p}$ is the pulse width, $f_{p}$ is the repetition rate and $E_{p}$ is the pulse energy

At higher energy, it is expected that the plume will have a higher overall temperature, due to the fact that more energy can be transferred to the substrate thus allow for larger excitation of phonons. When the average plume temperature is high, the growth rate of the nanoparticles is high resulting in larger particles at higher plume temperatures. This is due to the temperature dependence of particle growth rates. Particle growth is expressed by the following equation:

$$
r_{\text {growth }}=\operatorname{Aexp}^{-\frac{Q}{k T}} \quad \text { (Equation 2-3) }
$$


Where $\mathrm{r}_{\text {growth }}$ is the growth rate, $\mathrm{A}$ is an independent coefficient, $\mathrm{Q}$ is the activation energy required to initiate particle growth, $\mathrm{k}$ is Boltzmann's constant, and $\mathrm{T}$ is the temperature. ${ }^{[2-49]}$ As the temperature of the system increases the growth rate will have an exponential increase, therefore by increasing the peak power, more energy is transferred to the substrate and the laser plume thus increasing the plume temperature and increasing the growth rate and the size of the formed particles. As a result, both the repetition rate and the pulse width of the laser pulses will have a significant effect on the particle size.

These results show that precise control the material structure of the individual nanospheroids as well as the morphology of the nanospheroids and nanoweb layer cane be achieved by manipulating the interactions between the ultrafast laser pulses and the substrate material. As such direct regulation over the spheroid formation mechanism within the ion plume is possible. The Raman characteristics of the formed nanoweb layer are dependent on several different sources, each of which can be manipulated by controlling the nanospheroid formation mechanism.

Another of the critical features of the formation mechanism that proposed in this study, is the presence of polycrystalline grains and amorphous grains within individual nanospheroids. To confirm the crystalline/amorphous grains within the nanospheroids, TEM imaging of dispersed nanospheroids is required to determine crystal orientations and amorphous regions.

\subsubsection{SERS Enhancement from hybrid nanostructures}

\subsubsection{Raman Enhancement from hybrid nanostructures:}

The SERS and Raman enhancement from this unique nanomaterial is two-fold; the hybrid structure of the individual nanospheroids, as well as the physical morphology of the nanospheroids on the substrate surface contributes to the detection of the analyte. The sum of these contributions leads to the observed enhancement of the Raman signal of the silicon or any deposited analyte. To quantify this enhancement, the following equation was used.

$$
E F_{\text {nanostructure }}=\frac{I_{\text {nanostructure }} / N_{\text {surface }}}{I_{\text {substrate }} / N_{\text {bulk }}} \quad(\text { Equation } 2-4)
$$

Where $E F_{\text {nanostructure }}$ is a proportionality that quantifies the Raman enhancement between the substrate and the nanostructures by comparing the intensity of the peaks at a characteristic Raman shift of the analyte for both the substrate $I_{\text {substrate }}$ and the nanostructured surface $I_{\text {nanostructure }}$. 
The $E F_{\text {nanostructure }}$ proportionality requires a factor that takes into account the interaction volume of the Raman laser and the number of dye molecules within this volume which contribute to Raman enhancement; these factors are $N_{\text {surface }}$ and $N_{\text {bulk }}$ for the nanostructures and substrate, respectively. $N_{\text {surface }}$ takes into account the effective surface area of the nanostructures and the absorption of dye into the nanoweb structure within the Raman interaction volume. Some assumptions were made when calculating $N_{\text {surface }}$ and $N_{\text {bulk }}$; the density of the nanostructures is assumed to be identical to crystalline silicon, the dye absorption and surface area are assumed to be similar to the values established by Maznichenko et al. ${ }^{[2-46]}$ due to the same ion plume formation mechanism is employed to create both the $\mathrm{TiO}_{2}$ and hybrid $\mathrm{Si}$ nanostructures.

\subsubsection{Raman Results for bare substrates:}

A schematic of this portion of the experiment, the Raman spectra of nanoweb structures compared to a bulk Si wafer substrate spectra, the Raman spectra of each nanoweb structure with zoomed spectra for the $520 \mathrm{~cm}^{-1} \mathrm{Si}$ peak, and the enhancement factors for each substrate are demonstrated in Figure 2-7.

\subsubsection{Raman Results for dye-coated substrates:}

A schematic of this portion of the experiment with a molecular dye, the Raman spectra of the dyecoated substrates, the Raman spectra of each nanoweb structure with zoomed spectra for the 1360 $\mathrm{cm}^{-1} \mathrm{R} 6 \mathrm{G}$ peak and for the $1621 \mathrm{~cm}^{-1} \mathrm{CV}$ peak at both $10^{-3} \mathrm{M}$ and $10^{-6} \mathrm{M}$ concentrations, and the enhancement factors as function of peak power for each concentration on the nanoweb substrates are shown for both R6G (@ 1360 $\left.\mathrm{cm}^{-1}\right)$ and CV $\left(@ 1621 \mathrm{~cm}^{-1}\right)$ in Figure 2-8. 


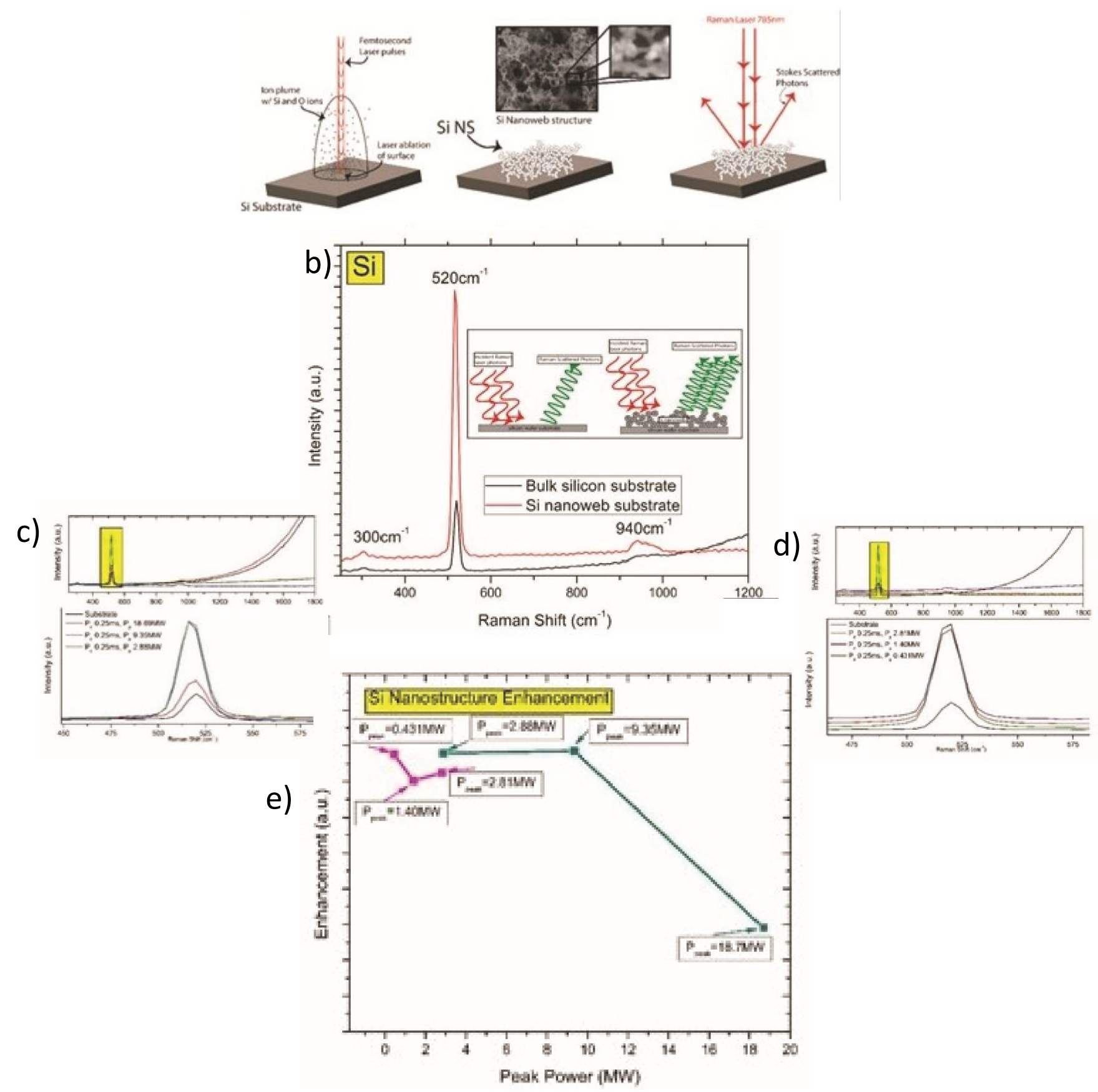

Figure 2-7: a) Schematic of Raman enhancement of bare Si nanowebs, b) Raman spectra silicon peaks on Si nanoweb and Si wafer substrate, Raman spectral peak $520 \mathrm{~cm}^{-1}$ for $\mathrm{Si}$ on $\mathrm{Si}$ nanowebs formed at c) low peak power and d) high peak power, and e) EF values at $520 \mathrm{~cm}^{-1}$ for Si on Si nanowebs as a function of Peak Power 

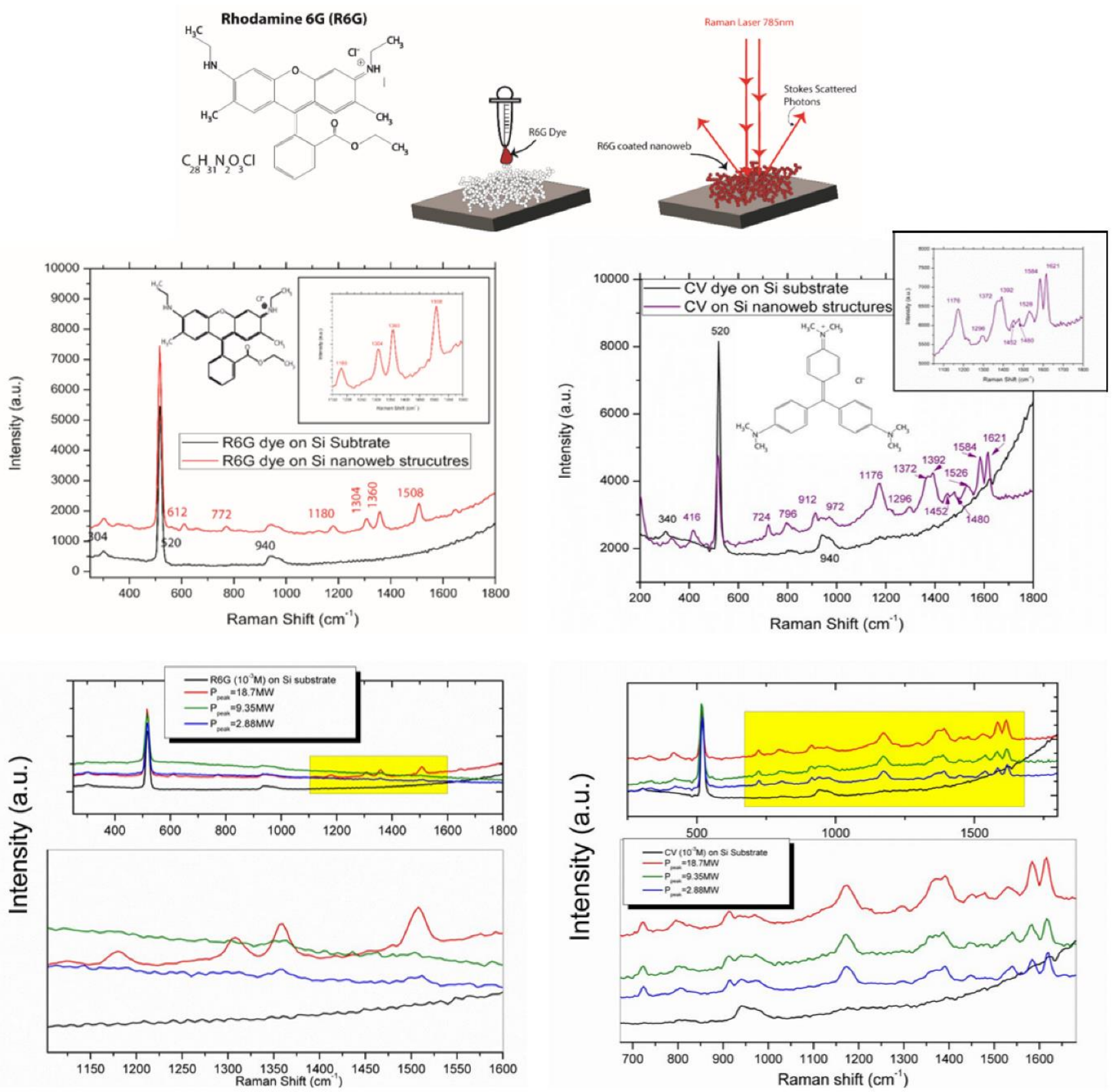

Figure 2-8: a) Schematic of Raman enhancement sensing with a molecular dye on Si nanowebs, Raman spectra of b) R6G and c) CV dye on Si nanoweb and Si wafer substrate, Raman spectra for d) R6G and e) CV on Si nanowebs for each laser ion plume condition 


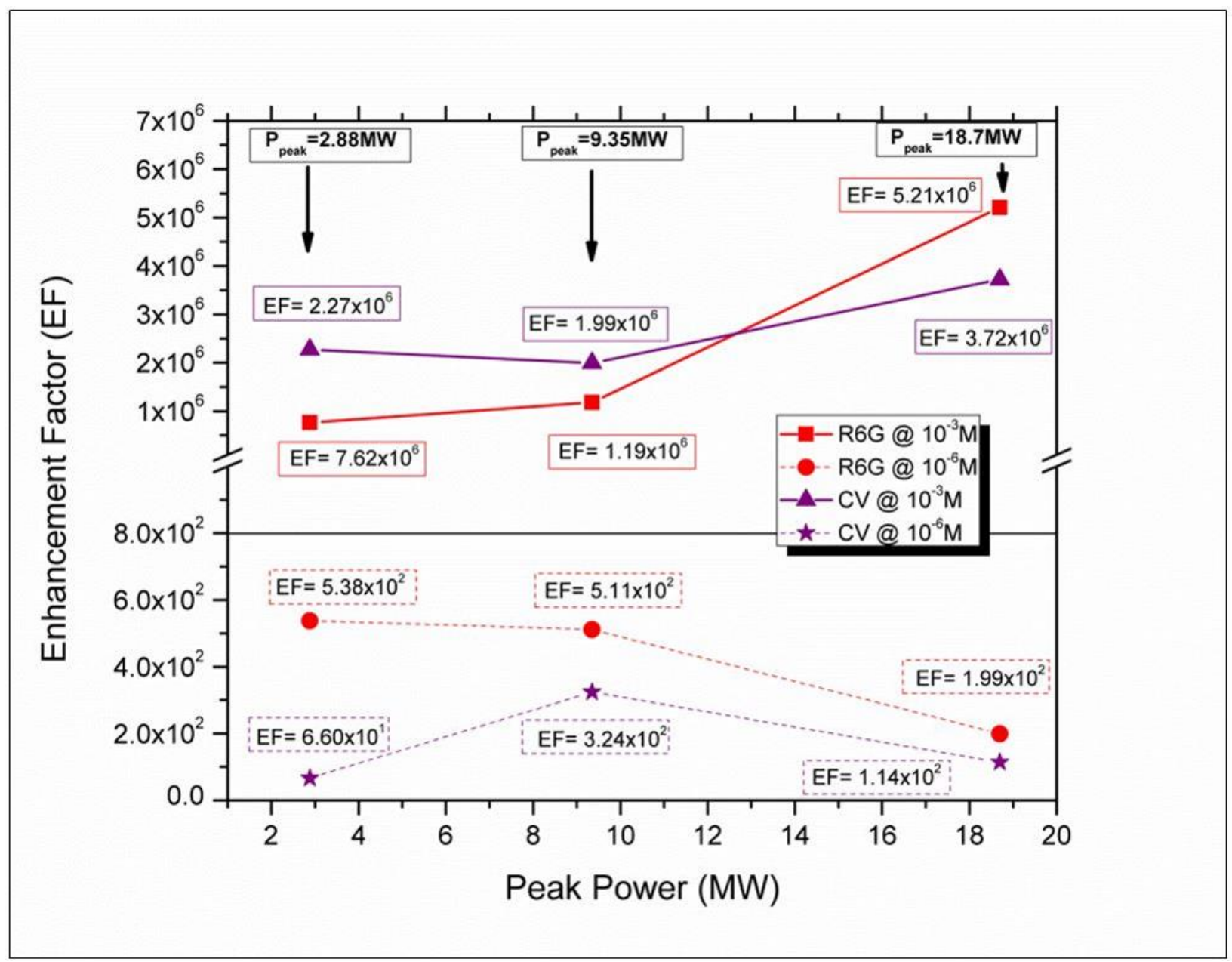

Figure 2-9: EF values for R6G and CV dyes on Si nanoweb structures at $10^{-3} \mathrm{M}$ and $10^{-6} \mathrm{M}$ concentrations as a function of peak power

From the Raman spectra and EF values (Figures 2-8, 2-9), it has been observed that a significant enhancement in intensity of the characteristic Raman peaks of both dyes on the nanoweb layer substrates compared to dyes on the Si substrate. When coated with the either the R6G dye or the $\mathrm{CV}$ dye, it has been observed that the bulk Si substrate spectra has no response to the presence of a dye; however when a dye is coated onto the nanoweb layer substrates, the characteristic peaks of the associated dye are clear well-defined. A maximum enhancement of $5.21 \times 10^{6}$ and $3.72 \times 10^{6}$ for both $\mathrm{R} 6 \mathrm{G}$ and $\mathrm{CV}$ dyes respectively are observed at $10^{-3} \mathrm{M}$ concentration at a peak power of 18.7MW. For $10^{-6} \mathrm{M}$ concentration of both R6G and $\mathrm{CV}$ the EF values while several orders of magnitude lower than the EF values at higher dye concentration, the Si nanoweb structures there remains observable enhancement of each dye spectra. This lends credence to the theory that this new nanomaterial is highly Raman active and is able to detect an analyte much more readily than 
bulk silicon. While it has been proven that roughened noble metal substrates exhibit SERS enhancement ${ }^{[2-47]}$ compared to noble smoother substrates, these results show that the Si nanoweb structures alone are able to enhance the detection of a molecular dye without the aid of noble metal SERS enhancement.

\subsubsection{Semiconductor SERS enhancement}

Due to current theoretical advances, the observed SERS enhancement can be attributed to a series of linked resonances only possible with semiconductor nanomaterials. ${ }^{[2-32-2-35]}$ The resonances that work in tandem to achieve an observed EF are surface plasmon resonance, molecular resonance, charge-transfer resonance and exciton resonance.

\subsubsection{SPR contributors: Hybrid Structure enhancement}

The amorphous/crystalline hybrid structure of the individual nanospheroids provides a distinct source of Raman enhancement due to its unordinary structure. The hybrid nanograin structure of the nanospheroids results in a high concentration of grain boundaries between grains with different orientations of crystalline silicon ([111], [220], [311]) and grain boundaries between crystalline silicon grains and gains of amorphous silicon within each nanospheroid. With TEM imaging, FFT analysis and XRD spectra, it has been observed that the presence of grains of multiple orientations of crystalline silicon and amorphous silicon in each nanospheroid. The grain boundary concentration affects how the light from the Raman laser scatters within the nanospheroid structure. Veprek et al., have demonstrated that grain boundaries in nanocrystalline silicon films exhibit enhancement in Raman scattering intensity, but is limited to processes that involve coupling of the electromagnetic field via charge density fluctuations in the grain boundaries to the bulk of the crystallites. ${ }^{[2-50]}$ Due to the bond stretching and compression (bond dilation) that occurs at the grain boundaries, a local electric dipole moment is formed which results in enhanced coupling with the EM field. Therefore with larger grain boundary concentration, the stronger the coupling with the EM fields thus more Raman enhancement. At higher peak power, a greater amount of amorphous content is present within the nanospheroids which can be inferred to as a greater concentration of grain boundaries; the nanoweb substrates created at higher peak power have the largest observed Raman enhancement of all the formed nanoweb structures in this study. 


\subsubsection{SPR contributors: Nanospheroid Size and Nanogap Enhancement}

The material structure of the nanospheroids is not the only source of Raman enhancement for our material, the nanospheroid size and the assembly of nanospheroids on the substrate surface provide enhancement as well. By changing the laser parameters to control the peak power, the spheroid size distribution of the silicon nanospheroids within the nanoweb can be adjusted. Figure 2-10 shows the nanospheroid size distributions for different nanoweb structures.
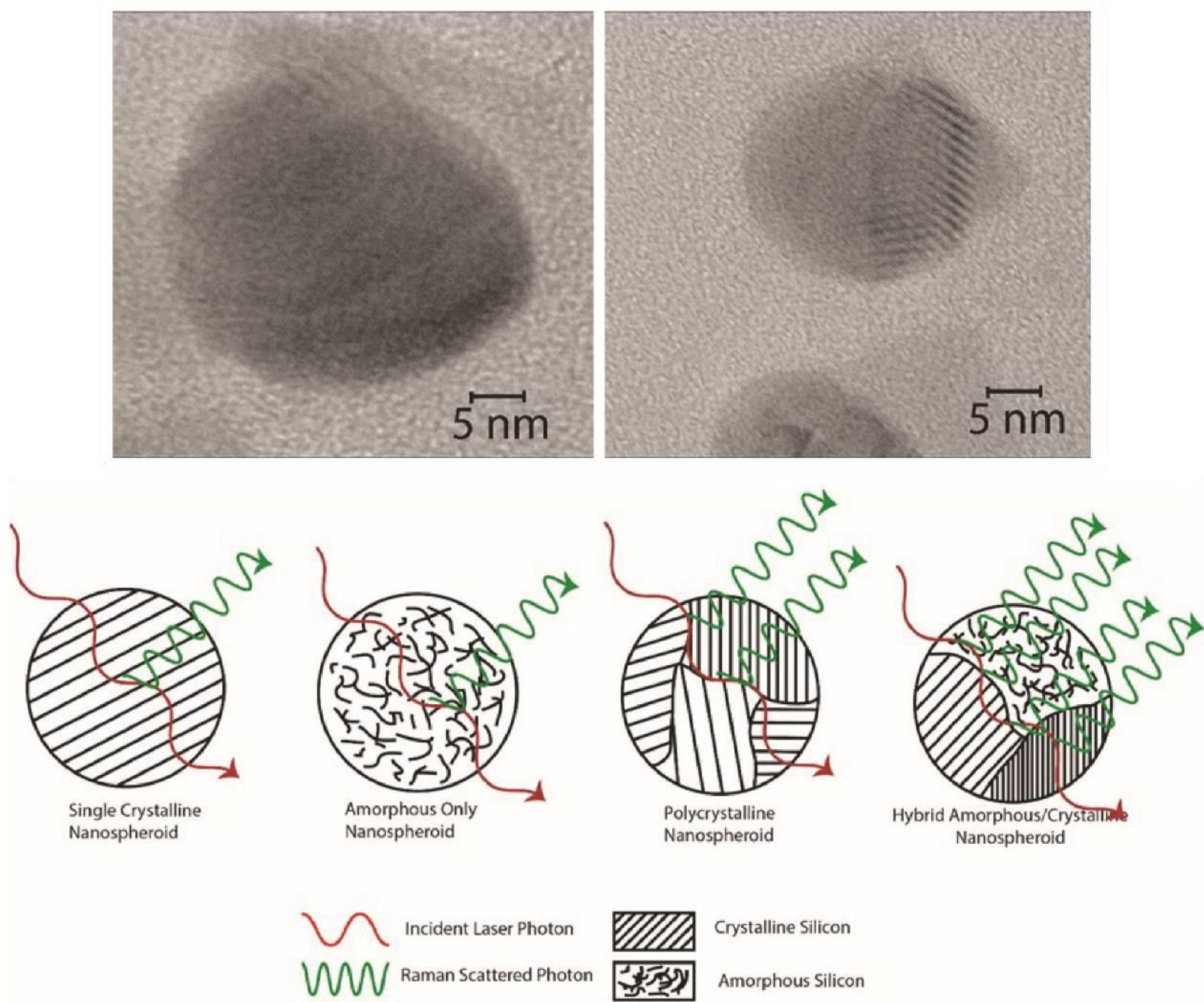

Figure 2-10: a) HRTEM images of Hybrid Amorphous/Crystalline nanospheroids, and b) a schematic of Grain Boundary Raman Scattering within a Single Crystalline Nanospheroid, an Amorphous Only Nanospheroid, a Polycrystalline Nanospheroid and a Hybrid Amorphous/Crystalline Nanospheroid 
Figure 2-11 shows that as the peak power decreases, the distribution of nanospheroid sizes broadens and the median value shifts to larger spheroid size. This result is congruent with the knowledge of the femtosecond lasers effect on the plume temperature, and the condensation and grain growth of nanostructures. The Raman spectra enhancement factors for the R6G dye and CV dyes on the silicon nanowebs (Figure 2-11) indicate that as the distribution of nanospheroid size increases and an effectively larger volume of large nanospheroids is generated, the enhancement of the dye peaks diminishes; therefore at large peak power, larger nanospheroids which cause greater enhancement of the dye molecules. This correlation however requires further study because not only do the nanospheroids decrease as peak power increases but the nanogap size decreases as well. (Figure 2-11)

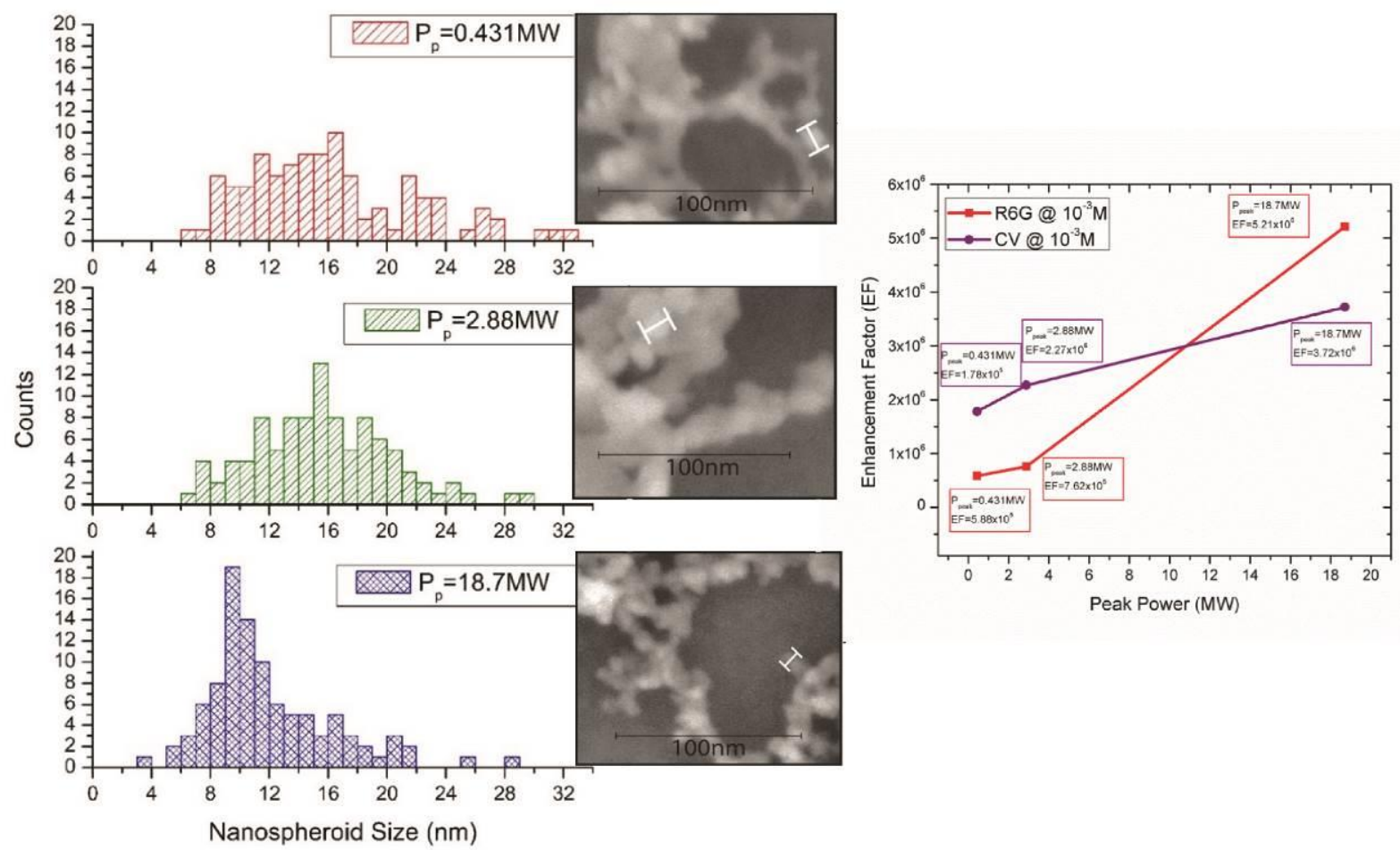

Figure 2-11: a) Nanospheroid size distribution and b) Enhancement Factor as a function of peak power at $0.431 \mathrm{MW}, 2.88 \mathrm{MW}$ and $18.7 \mathrm{MW}$

\subsubsection{SPR contributors: Nanogap/Raman hot-spot enhancement}

It has been shown and well established, that the size of the nanogaps between Raman active nanostructures has a direct and crucial influence on the Raman enhancement factor of a material. 
The unique structure of the nanoweb layer adds additional enhancement effects due to the formation of nanogaps between individual nanospheroids. These nanogaps are formed when the $\mathrm{Si}$ nanospheroids fuse together on the substrate surface to create fibre-like structures. These nanogaps allow for a concentration of electromagnetic field which allows for an amplification of the SERS enhancement. ${ }^{[2-51]}$ It has been shown that when the nanogap size is significantly reduced, an order of magnitude enhancement is observed. ${ }^{[2-20,2-43,2-52]}$ The nanogaps for three different nanoweb layers each created at different peak powers have been measure and the nanogap size distributions are displayed in Figure 2-12.

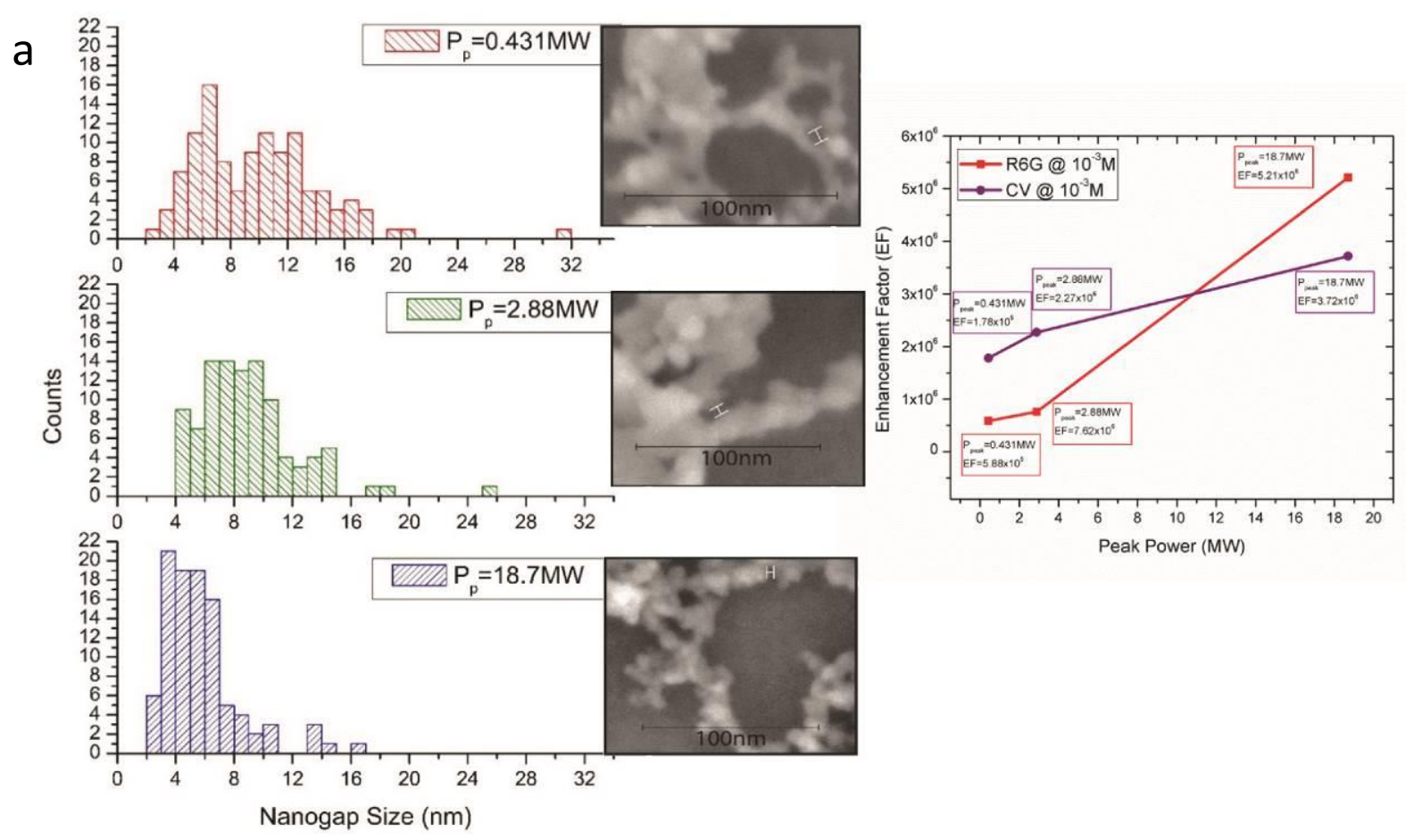

Figure 2-12: a) Nanogap size distribution and b) Enhancement Factor as a function of peak power for nanowebs created at $0.431 \mathrm{MW}, 2.88 \mathrm{MW}$ and $18.7 \mathrm{MW}$

These distributions clearly show that as the peak power is reduced the nanogaps between the nanospheroids increase in size and become less uniform. This increase in size correlates to a decrease in Raman enhancement seen in Figure 2-12. The maximum EF is obtained at a peak power of $18.7 \mathrm{MW}$ and the median nanogap size observed with this specific nanoweb is approximately $5.15 \mathrm{~nm}$. While this value is dissimilar to reported optimal nanogap sizes ${ }^{[2-53]}$ for other SERS active nanomaterials ${ }^{[2-51-2-58]}$, this hybrid nanoweb material has a distinction in material character and a contribution to EF by the grain boundary scattering of the individual 
nanospheroids, which divorces currently established values for optimal nanogap size for noble metal nanostructures from our truly silicon based Raman active material. The role of the nanogap distribution also plays an important role in the SERS enhancement ${ }^{[2-59-2-61]}$; the nanogap distribution observed in Figure 2-12 shows clear evidence of a broadening of nanogap size as peak power increases. While the observed nanogap size cannot be held to a standardized value for other nanomaterials, the principle governing Raman enhancement from a narrow distribution of nanogap sizes ${ }^{[2-53]}$ can still be applied to the nanostructures. As observed from Figure 2-12, the enhancement factor decreases as the breadth of the nanogap distribution increases. This is due to a lack of efficiency in Raman scattering from the nanogaps ${ }^{[2-62]}$ and a smaller concentration of nanogaps within the optimal range for our hybrid Si nanomaterial.

\subsubsection{Molecular, Charge-Transfer, and Exciton Resonance Contributors}

Lombardi and Birke ${ }^{[2-40]}$ have established a comprehensive theory to describe and predict the SERS scattering in semiconductor materials. Their theory states that observed SERS enhancement with semiconducting nanomaterials are not mainly attributed to SPR, as in the case of metallic nanomaterials, but also ascribed to linked resonances between molecular resonance, chargetransfer resonance and exciton resonance. Their theory also suggests that not only are semiconductors capable of achieve EF values comparable to those of noble metal nanostructures, but semiconductor nanomaterials have the capacity to attain single molecule sensitivity for chemical sensing applications.

The contribution to SERS enhancement from molecular and charge transfer resonance occurs when the valence and conduction bands of the semiconductor nanomaterial are similar in energy level to the lowest unoccupied molecular orbital (LUMO) and highest occupied molecular orbital (HOMO), respectively. When this similarity in energy level occurs, a charge transfer can occur between the valence band edge and the LUMO or the conduction band edge and the HOMO resulting in a SERS enhancement, the most intense of which occurs when transitions terminate at band edges. In addition exciton resonances occur when excitons (electron-hole pairs) created in the semiconductor nanomaterial by optical absorption. These excitons created form exciton levels from below the conduction band to above the valence band which forms a sequence in the absorption spectra. When the nanoparticle size decreases below the Bohr radius for the semiconductor material, the exciton levels diverge, due to quantum confinement, leading to a 
dependence of the SERS enhancement spectra on the semiconductor nanoparticle size. Each of the contributions to semiconductor SERS enhancement will be explored as research topic with the further development of these hybrid Si nanostructures.

\subsection{Summary}

In this study is has been demonstrated the unique ability to create a Raman active nanomaterial from an inactive Raman bulk material. This silicon based Raman active material, is uniquely able to increase the cross-section of Raman scattered photons without the use of well-established SERS active nanomaterials. Not only can the Raman sensitivity be activated with the nanoweb material, the formation mechanism can precisely tune the Raman activity of this nanomaterial by controlling the ion plume formation mechanism, by controlling the laser/substrate interaction parameters. The material formed is a nanoweb material composed of an interconnected network of hybrid amorphous/crystalline nanospheroids. The hybrid nature of the individual nanospheroids and the nanogap concentration of the nanoweb structure and the linked resonances present in this SERS active Si nanoweb semiconductor material are the major contributors to the increase in Raman activity. It has also been proven that this nanomaterial has the potential to be used as a chemical sensing device with the observation of a maximum enhancement factor of $5.38 \times 10^{6}$ for chemical

dye R6G and $3.72 \times 10^{6}$ for $\mathrm{CV}$ dye. With these results, a new avenue for the use of silicon as a Raman active material for chemical sensing devices has been opened. 


\title{
Chapter 3
}

\section{Hybridized Enhancement of SERS Detection of Chemical and Bio-marker Molecules Through Au Nanosphere Ornamentation of Hybrid Amorphous/Crystalline Si Nanoweb Nanostructure Biochip Device}

\author{
Published in The Journal of Material Chemistry B, 2016
}

In this chapter, the fabrication of hybrid Si SERS nanobiosensor biochip device. The biochip exhibits a proposed hybridized SERS biomolecule sensing phenomenon with the creation of a SERS-active 3D amorphous/crystalline silicon nanoweb that has undergone nano-ornamentation with gold nanospheres. The underlying interconnected 3D hybrid Si nanospheroid network created by a laser-ion plume formation mechanism is nano-ornamented with gold nanospheres using a physical ion plasma deposition method to enhance the nano-Raman sensitivity of these nanostructures. It is observed that a tunable hybridized enhancement of analyte detection as a result of paired SERS mechanisms arising from a Raman active nano-hybrid Si nanoweb structure and $\mathrm{Au}$ nanosphere ornamentation. This hybridized enhancement has to date, yet to be reported for biosensing with SERS semiconductor materials. By linking the SERS enhancing effects of the base nano-hybrid Si nanoweb material with the SERS enhancing characteristics of gold nanospheres, a significant increase in sensitivity for the detection of chemical and bio-label molecules is observed. Through manipulation of the hybrid Si nanoweb structure formation in coordination with modulation of the nano-ornamentation process, hybrid nanostructured SERSactive nanomaterial can be tailored specifically for biosensing applications. A calculated enhancement factor (EF) for chemical molecule Crystal Violet (CV) of 3.0x10 7 and an EF value for bio-label molecule Rhodamine 6G (R6G) of $1.4 \times 10^{7}$ taking an Au nanosphere ornamented Si wafer as a benchmark.

\subsection{Introduction}

Biosensing is a broad field of research where multiple avenues of detection are used for the same applications and analytes. Among these sensing mechanisms, which include electrochemical sensing ${ }^{[3-1]}$ field-effect transistor (FET) sensing ${ }^{[3-2]}$, and piezoelectric sensing ${ }^{[3-3]}$, optical sensing 
includes several powerful and versatile techniques ${ }^{[3-4,3-5]}$ that are currently attracting a large amount of scientific interest. Optical sensing techniques can provide data regarding a bio-analyte with extreme sensitivity and precision while being a non-destructive and cost effective analysis tool ${ }^{[3-6]}$. Of the techniques that are classified as optical sensing mechanisms, Raman sensing is one of the most valuable due to the depth of information provided by a Raman spectra, as well as the potential sensitivity that can be attained through enhancing a Raman bio-transducers' sensing potential with the use of nanostructured materials ${ }^{[3-7,3-8]}$.

The use of nanostructured materials for enhancing the nano-Raman sensitivity for bio-analyte detection is a field of research that has become well-established and grown profoundly with recent advances. $^{[3-9-3-11]}$ Surface enhanced Raman scattering (SERS) enhancement techniques have allowed for a unprecedented increase in the sensitivity of detection across a vast array of applications; not only are SERS active materials able to enhance the detection and characterization of chemical analytes ${ }^{[3-12-3-14]}$, but these materials can be utilized for detection of explosive materials ${ }^{[3-15]}$, pharmaceuticals ${ }^{[3-16]}$, water pollutants ${ }^{[3-17,3-18]}$, and a large variety of biomolecules ${ }^{[3-19-3-23]}$, all at very low concentrations ${ }^{[3-26-3-29]}$ with the possibility of single molecule detection an obtainable goal in some cases. ${ }^{[3-28-3-30]}$

It has been shown that noble metal nanostructures (NMNs) can play key role in the SERS enhancement for analyte detection and in many cases, provides the singular source of Raman scattering enhancement. ${ }^{[3-31-3-33]}$ NMNs such as nanoparticles ${ }^{[3-10,3-34-3-36]}$, nanostars ${ }^{[3-37,3-38]}$, and nanorods ${ }^{[3-39-3-42]}$ have proven enhancement characteristics due to the phenomena known as surface plasmon resonance (SPR). This phenomenon is unique to these gold and silver NMNs because noble metal surface plasmons become resonant when excited by photons within the wavelength range used for Raman lasers $(532-785 \mathrm{~nm})$. As a result, the level of observable SERS enhancement from NMNs has been reported with enhancement factors (EF) that range from $10^{6}$ ${ }^{[3-43]}$ to $10^{10}{ }^{[3-44]}$. In many cases these NMNs have been able to have limits of detection from $10^{-}$ ${ }^{14} \mathrm{M}$ analyte concentrations ${ }^{[3-45]}$ to near single molecule detection ${ }^{[3-28]}$.

However, noble metal only SERS sensing nanomaterials have several severe limitations that cause these materials to be undesirable in many applications. Many methods that are commonly used to form these NMNs are unable to create a consistent particle size which is necessary for a stable SPR enhancement and for reproducibility of SERS signal. ${ }^{[3-46]}$ Moreover, noble metal substrates 
can be highly expensive to fabricate and have undesirable biocompatibility, which limits their use as sensing materials for low-cost sensing platforms and for the detection of many biomolecules. ${ }^{[3-}$ ${ }^{47]}$ As a result, the need for SERS active nanostructured materials that have reproducibility of SERS enhancement as well as strong biocompatibility is paramount for biosensing applications.

Recent theories and developments ${ }^{[3-46]}$ have shown that NMNs are not the only materials that can provide significant enhancement in Raman sensitivity. There have substantial advances towards using semiconductor nanostructures as a primary source of SERS enhancement. Semiconductor nanomaterials such as $\mathrm{TiO}_{2},{ }^{[3-3,3-48]}, \mathrm{NiO}^{[3-49]}, \mathrm{GaP}^{[3-50]}, \mathrm{CuTe}^{[3-51]}$ and $\mathrm{ZnSe}^{[3-52]}$ have observable enhancement factors above 106, EF values that are competitive with NMNs in terms of SERS intensity. Lombardi and Birke ${ }^{[3-54]}$ have theorized that, rather than SPR being the primary source of Raman enhancement in semiconductor materials, semiconductor SERS occurs due to a series of linked resonances that each contribute to the enhancement of analyte detection. These resonances include exciton resonance (electron-hole pairs created by optical excitation causing changes in quantum confinement of the nanomaterial), plasmon resonance (collective oscillation of electrons on the surface of the nanomaterial) and charge-transfer resonance (the transfer of an electron from the valence band of the semiconductor to the lowest unoccupied molecular orbital of the analyte molecule).

While current research in semiconductor SERS nanomaterials for biosensing applications is progressive, the nanostructures of these Raman active semiconductors remain similar to traditional SERS NMNs. The works by Jiang et.al ${ }^{[3-54]}$ Chang et.al ${ }^{[3-55]}$ and Livingstone et.al ${ }^{[3-56]}$, each report large enhancement of analyte detection with $\mathrm{Cu}_{2} \mathrm{O}$ nanospheres, $\mathrm{ZnMgO}$ nanoparticles, $\mathrm{CdSe} / \mathrm{ZnBeSe}$ quantum dots respectively. These nanostructures are very similar to those used by other NMN research groups. The development of new 3D semiconductor-based nano-architectures remains vastly under researched and has the potential to provide enhancement for SERS biosensing not achievable with 1D nanoparticle enhancement. Research regarding 3D nanostructures for SERS activation is mainly isolated to top-down approaches with limited enhancement ${ }^{[3-57]}$ or to 3D nanostructures that use NMNs as primary enhancement sources. ${ }^{[3-59-3-61]}$ Bottom-up SERS active nanomaterials are mostly limited to noble metals only. ${ }^{[3-62-3-64]}$ By using a laser-ion plume mechanism, it is possible to generate a hybrid-Si nanomaterial that has highly 3D nanostructure; the ion-plume formation mechanism creates an interconnected nanonetwork of branched fused- 
nanospheroids from a standard $\mathrm{Si}$ wafer substrate. To our knowledge this type of hybrid-Si nanoweb material has yet to be used by other research groups for use as a highly active SERS biosensing platform. In the previous study ${ }^{[3-64]}$ it has been observed that an enhancement of SERS sensing using a unique semiconducting hybrid amorphous/crystalline Si nanoweb material, with a maximum EF of $10^{6}$, a level enhancement not observed with other Si-only nanomaterials. ${ }^{[3-65-3-}$ 67]

With this current research, a significant increase in SERS enhancement of bio-molecule analytes with the hybrid Si nanoweb material is achieved by exploiting both the SPR from NMNs and the linked resonance enhancement of the underlying Raman-active semiconductor nanostructure, a hybrid crystalline/amorphous Si nanoweb material. While others researchers develop a biosensing platform through the modification of pre-existing or chemically synthesized nanomaterials ${ }^{[3-68,3-}$ ${ }^{69]}$ in order to achieve a large EF, in this study the synthesis of a SERS biosensing nanomaterial from a base Si wafer and with subsequent nano-ornamentation with Au nanospheres, the creation of a biochip nanomaterial that can easily be introduced into a nanobiosensor fabrication process to greatly enhance the sensing capabilities of biosensing devices. In this study, using a laser-ion plume formation mechanism to form the nano-hybrid Si nanoweb material and an ion-plasma physical deposition method for ornamenting the interconnected hybrid Si nanospheroid network with gold nanospheres, a SERS enhancement that exceeds that of the nano-hybrid Si nanoweb alone has been achieved. By varying the ion formation environment within the laser-ion plume, precise control of the creation of a hybrid Si 3D nanoweb architecture as well as the composition of the individual hybrid-Si nanospheroids that comprise this nanostructure is reported; control over nanostructure formation is required in order to activate the linked resonance enhancement mechanisms that makes this a SERS sensitive material for biosensing applications ${ }^{[3-64]}$. By ornamenting this nanoweb structure with $\mathrm{Au}$ nanospheres, the SPR enhancing phenomena is employed to further increase the SERS enhancement of biomolecules with the hybrid Si nanoweb structure. It is proposed that these two mechanisms work harmoniously to increase the intensity of bio-analyte spectra.

The goal with this study is to establish an Au nanosphere ornamented hybrid Si nanoweb material as a biochip sensing platform for chemical and bio-sensing applications through potential integration into with a biosensor device. The effects of both the linked resonance SERS 
enhancement and the SPR SERS enhancement have on the sensing of chemical and bio-label molecules are studied. In addition, the dynamic nature of the laser-ion plume formation and the nano-ornamentation process to create a nanomaterial, displays a harmonization of the linked resonance enhancement and the SPR enhancement mechanisms for increased bio-analyte detection. This new nanomaterial on a Si wafer becomes a semiconductor-based SERS biochip that can offer compatibility with existing sensor fabrication techniques. With these results the establishment of this biosensing nanomaterial as a highly SERS-active biochip sensing platform for many biosensing applications is realized. Figure 3-1 shows a schematic of the overall experimental concept for this study.

\section{Biochip Fabrication Process}

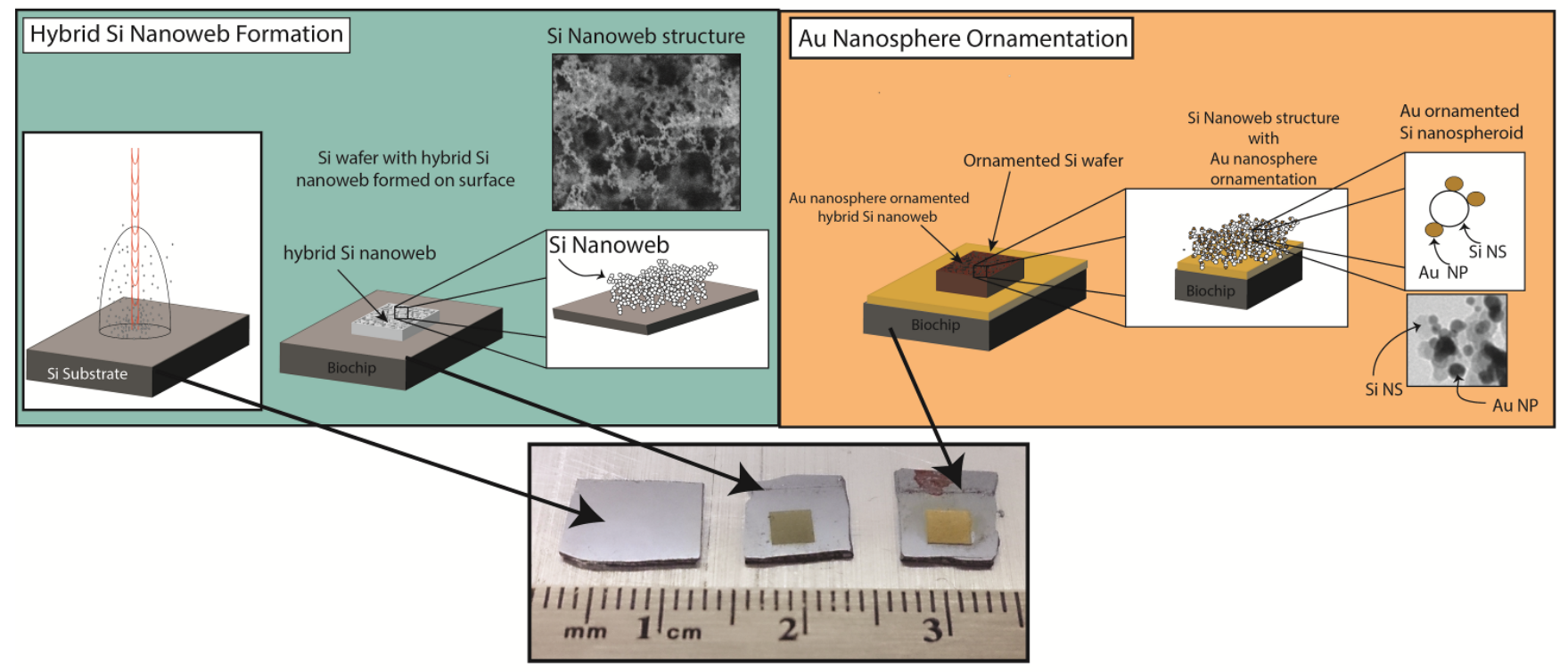

Figure 3-1: Overall concept of SERS-active biosensing nanomaterial study; a hybrid Si nanospheroid network ornamented with Au nanospheres as a SERS biochip sensor 


\subsection{Experimental Method}

For the creation of the underlying hybrid Si nanoweb structures, a Clark-MKR IMPLUSE pulsed Yb-doped fibre amplified femtosecond laser was used to ionize the $0.02-\Omega \mathrm{cm}$ p-type silicon (100) wafer substrate. To take full advantage of the control over the nanoweb fabrication in this experiment, the laser wavelength $(1030 \mathrm{~nm})$, polarization (circular), dwell time $(0.5 \mathrm{~ms})$ and the laser power $(16 \mathrm{~W})$ were kept static. The varied laser parameters in this study were the repetition rate and pulse width, with which the laser fluence $\left(\mathrm{F}_{\text {laser }}\right)$ of the laser ion plume can be controlled. The nanoweb structures were generated using a 300x300 point array with 25 micron point spacing to create a uniform distribution of nanostructures on the base Si wafer; using EzCAD software this array was designed and a piezo-driven raster was used to move the laser beam across the substrate surface.

The formed nanostructures were ornamented using a physical deposition method; a 99.9\% pure Au target was bombarded with ion particles with an atmosphere of Argon gas to ionize the Au target. The Au ions then physically deposit onto the surface of the nanoweb structures for a set time. Three deposition times as specified by the sputter coating device, $15 \mathrm{~s}$ (short), 30s (moderate) and 45s (long) are used to increase the amount of ornamentation on the surface of the $\mathrm{Si}$ nanostructures.

A Raman spectrum was taken on each nanostructure before and after coating with analyte solutions using a Bruker Optics SENTERRA Raman microscope. A Raman laser wavelength of $785 \mathrm{~nm}$ at a power of $25 \mathrm{~mW}$ with 3 iterations at and integration time of 10 s to obtain the Raman spectra and to ensure repeatability of the spectral results. For the first experiment set, the chemical dye used to test the enhancement capability of the Au-NP ornamented nanoweb structures was crystal violet $(\mathrm{CV})$, which is a common dye for SERS analysis due to its large Raman cross-section. For the biolabel sensitivity experiment set, rhodamine $6 \mathrm{G}(\mathrm{R} 6 \mathrm{G})$ was applied to each nanoweb surface at varying concentrations. For $\mathrm{CV}$ a single drop of the dye at $10^{-3} \mathrm{M}$ was applied to a separate nanostructured area and for R6G a single drop of at $10^{-6} \mathrm{M}$ bio-label was applied to each nanoweb area prior to spectral analysis. 
For characterization of the ornamented nanoweb structures, High-resolution tunneling electron microscopy (HRTEM) imaging was used to determine the physical morphology of the nanoweb structures and to analyze the size of the Au-NP ornamenting the nanospheroids.

X-ray photoelectron spectroscopy (XPS) was used to determine the elemental composition of the nano-ornamented Si nanowebs and the relative amount of gold ornamented on the surface of the nanoweb structure. The XPS data was collected using a Thermo Fisher K-Alpha XPS system using a monochromated Al K-alpha X-ray source with a 2:1 ellipse spot size was $400 \mu \mathrm{m}$ as the major axis Regional scans were performed at $50 \mathrm{eV}$ pass energy and $0.1 \mathrm{eV}$ point spacing to give better energy resolution and peak shape definition. Using the Avantage software, the quantification of $\mathrm{Si}, \mathrm{O}$, and Au was obtained.

\subsection{Results and Discussion}

\subsubsection{Silicon Laser-Ion Plume Nanospheroid Biosensing Material Formation and Ornamentation}

As has been shown in previous research ${ }^{[3-70-3-73]}$, the formation of these nanoweb structures is unique to the laser-ion plume formation mechanism. The pulse energy provided by the femtosecond laser pulses readily surpasses the ionization threshold for the silicon wafer substrate allowing for immediate ionization of the solid crystalline silicon structure and the formation of an ion plume, containing $\mathrm{Si}^{+}$ions and $\mathrm{O}^{2-}$ ions which are created by the ionization of the ambient air surrounding the wafer substrate. Within the ion plume it has been theorized ${ }^{[3-64]}$ that, the $\mathrm{Si}^{+}$ions are amassing to form nanoclusters of amorphous or crystalline $\mathrm{Si}$, which then become ejected from the plume. These nano-aggregates then condensate and fall to the wafer surface as hybrid amorphous/crystalline nanospheroids, where upon these nanospheroids fuse together to form a nanoweb network structure on the material surface. Figure 3-2 shows a schematic of this formation mechanism. It has been shown ${ }^{[3-64]}$ that this nanomaterial has enhancing characteristics comparable to that of traditional noble-metal based SERS enhancing materials, by achieving an EF value on the order of $10^{6}$; the same laser-ion plume formation mechanism is employed in this study.

In this study, the ability to program the structure of the nanoweb material by modifying the laser ion-plume formation environment to tune the material for biosensing characteristics is observed. The nanomaterial structure and composition generated by the laser ion-plume formation 
mechanism are governed by several different contributing factors, those being the laser parameters including the repetition rate, pulse width, dwell time, spacing of points etc.; however speaking in terms of the plume energy, plume temperature and condensation rate within the plume itself is more useful for explaining how the material is formed and how this nanomaterial can be modified to allow for greater SERS enhancement. As such, it is necessary to evaluate the enhancing characteristics of this nanomaterial in terms of the material composition and the unique physical morphology of the nanoweb structure. Therefore a chosen selection of laser plume conditions that provide varying physical morphologies and material compositions in order to determine how these factors play a role in SERS enhancement for this nanoweb structure.

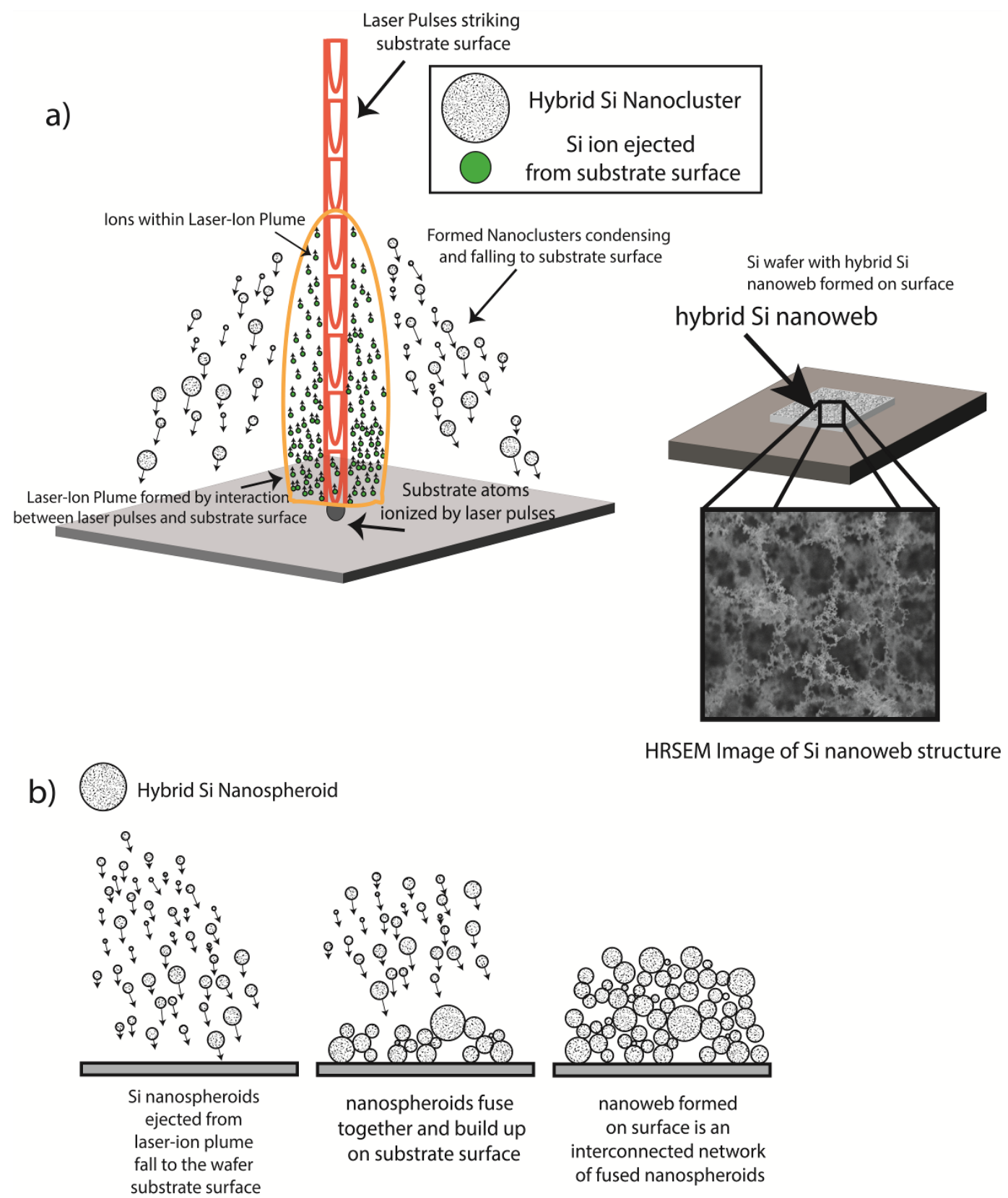


Figure 3-2: a) The hybrid crystalline/amorphous Si nanoweb formation by laser-ion plume formation mechanism and b) the deposition of Si nanospheroids onto Si wafer surface

\subsubsection{SERS augmentation through an Ornamentation Process}

A physical ornamentation process by which gold nanospheres are deposited onto the surface of the nanospheroid networks in order to take advantage of the SERS characteristics of NMNs and to generate a two-fold hybridized Raman enhancing nanostructure. The advantage of Au nanosphere ornamentation is the addition of a SPR contribution to the enhancement of the analyte spectra. Most semiconductor nanostructures do not exhibit significant SPR effects within the proximity of the Raman laser excitation range, but they do exhibit other enhancement mechanisms ${ }^{[3-53]}$. The goal of using Au nanospheres in this study is to determine how the enhancement of analyte spectra can be bolstered by creating a hybridized enhancement source. The ornamentation process used in this study is a sputtering process wherein the nanoweb substrates are placed within a HV chamber and subjected to an argon rich atmosphere. A current is passed through the chamber which ionizes the Ar molecules to form $\mathrm{Ar}^{+}$ions, while an Au target is negatively charged, causing the $\mathrm{Ar}^{+}$ions to bombard the $\mathrm{Au}$ target. This bombardment ejects $\mathrm{Au}$ nanospheres from the target molecule towards the nanoweb substrate leading to a physical deposition of these Au nanospheres onto the nanoweb surface; the amount of Au nanospheres that ornament the nanoweb surface is dependent on the amount of time the Au target is bombarded, referred to here as the ornamentation time (OT). Figure 3-3 is a schematic of this process with an HRTEM image of an ornamented nanoweb cluster. 
Nanoweb Biochip substrate

Hybrid Si nanoweb

w/ Au nanosphere ornamentation
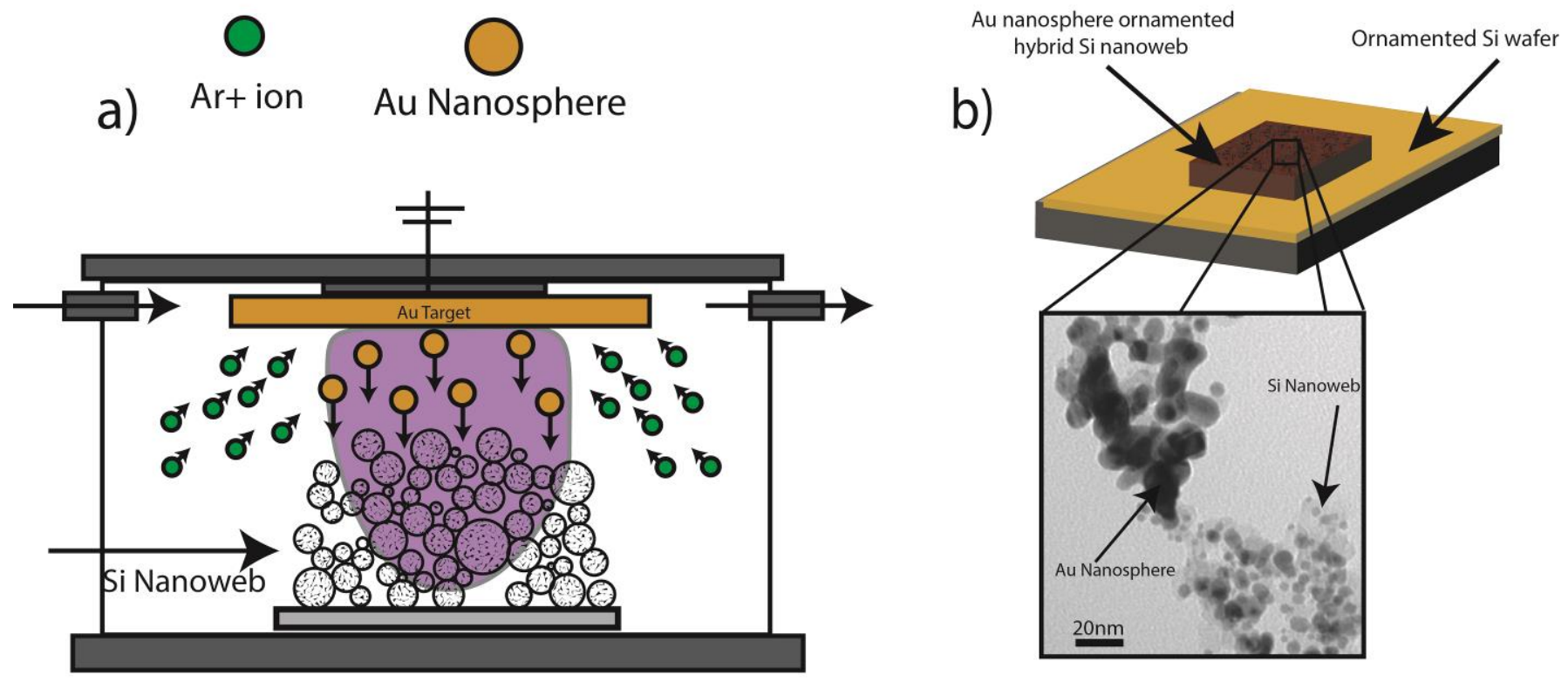

Figure 3-3: a) Ornamentation of hybrid Si nanoweb with Au nanospheres using physical deposition method with b) an HRTEM image of ornamented nanoweb material

Figure 3-4 shows the Raman spectra of each SERS biochip substrate and the EF values associated with these nanoweb structures. 

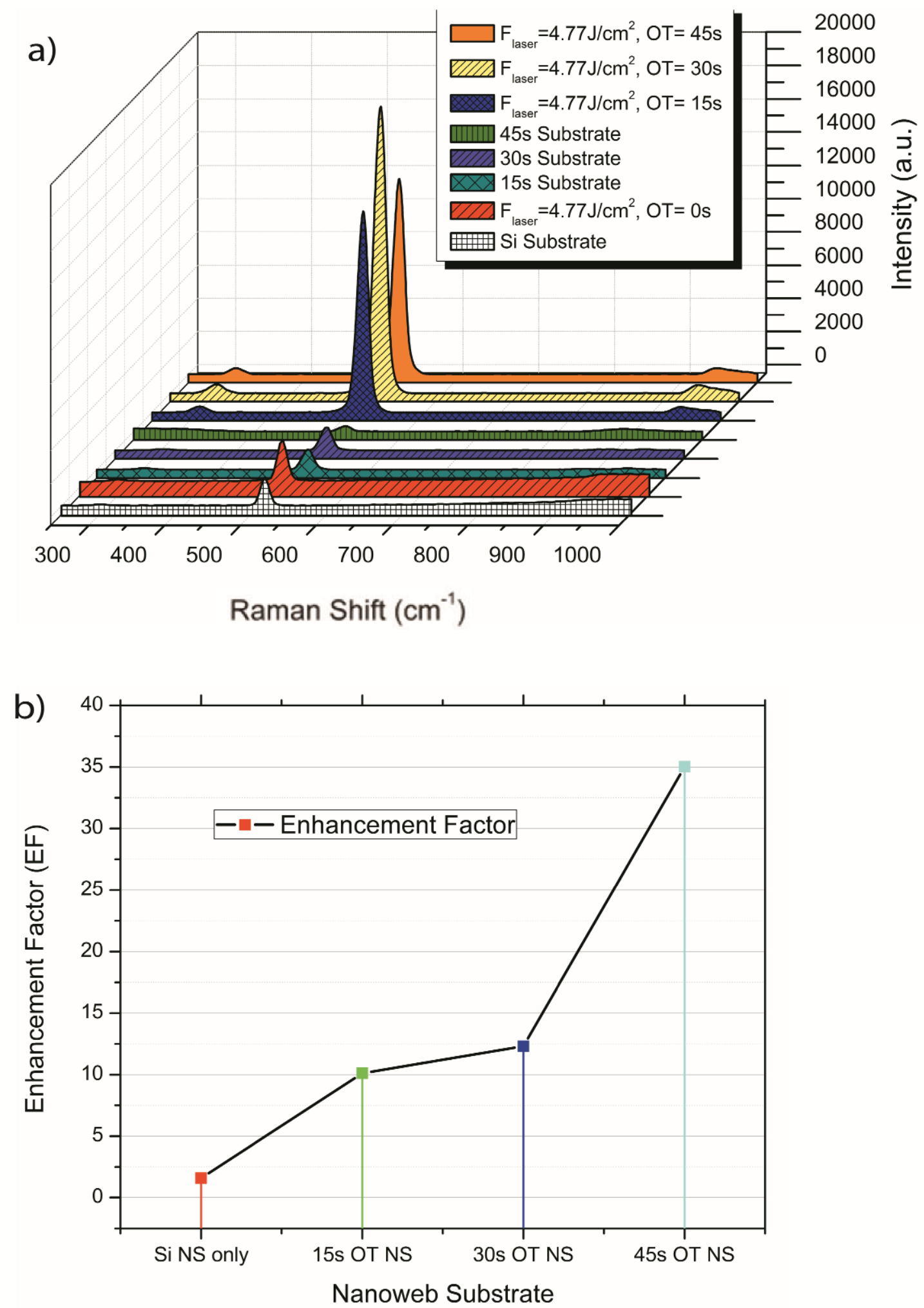

Figure 3-4: a) Raman spectra of ornamented hybrid Si nanoweb materials and b) EF values of each SERS biochip nanomaterial compared to bare $\mathrm{Si}$ wafer substrate 
It is clear from these spectra, that the hybrid $\mathrm{Si}$ nanoweb structure and the nanosphere ornamentation have a significant influence on the activation of Raman enhancing mechanisms. These spectra show that for the $520 \mathrm{~cm}^{-1}$ peak characteristic to $\mathrm{Si}$, when ornamented with $\mathrm{Au}$ nanospheres, becomes muted compared to the $\mathrm{Si}$ wafer and that compared to the nanoweb structures created from the Si wafer, this peak becomes substantially enhanced compared to the $\mathrm{Si}$ wafer itself, a result consistent with our previous studies ${ }^{[3-64,3-74,3-75]}$. The most interesting finding is that, this peak becomes even more enhanced for the Au ornamented nanoweb than just the nanoweb alone. This result signifies that not only is the nanoweb material able to cause a significant enhancement but the ornamentation of $\mathrm{Au}$ nanospheres is able to amplify this enhancement to a large degree, by a factor of 35 in the EF plot. It is postulated that there are multiple mechanistic sources at play that work cooperatively to allow for a hybridized SERS enhancement and that this multi-sourced enhancement will be able to greatly improve the detection of many chemical and bio-analyte molecules.

\subsubsection{Semiconductor and Metallic SERS Enhancement}

It has been well established by many other researchers that noble metal nanoparticles and nanostructures are able to drastically increase the SERS detection of analytes due to the electromagnetic enhancement mechanism known as surface plasmon resonance (SPR) ${ }^{[3-53]}$. With SPR as the sole SERS enhancing mechanism, researchers in this field have been able to observe EF values on the order of $10^{6}$ to $10^{10}[3-76,3-77]$. In addition, it has been theorized by Lombardi and Birke $^{[3-53]}$, with observations from several of their contemporaries, that semiconductor nanostructured materials can be used as powerful SERS sensing platforms in and of themselves. They have theorized that the SERS enhancement observed in these semiconductor nanomaterials is due to several linked resonances each contributing to the observed enhancement. These resonance contributors include exciton resonance, plasmon resonance and charge transfer resonance. $\mathrm{TiO}_{2}{ }^{[3-48]}, \mathrm{Sn}: \mathrm{ZnCdO}^{[3-78]}, \mathrm{W}_{18} \mathrm{O}_{49}{ }^{[3-79]}$ and other semiconductor nanomaterials have been observed to have enhancement factors of $10^{4}, 10^{5}$ and $10^{5}$ respectively. While NMNs have been coated or deposited onto semiconductor ${ }^{[3-80]}$ or metal oxide ${ }^{[3-81]}$ nanostructures in many studies, these semiconductor templates act only as scaffolding for the SERS active NMNs and are not considered as primary contributors to the observed enhancement. As a result, few substantive 
studies have been conducted to determine how SPR from NMNs and the linked resonances from the semiconductor nanostructures can be combined to further increase enhancement proficiency and detection sensitivity.

In this study, a formulated nanomaterial with two SERS active nanostructures are combined together to create a semiconductor based SERS biochip sensing material with a two-fold contribution to SERS detection sensitivity; these enhancements arise from SPR of the ornamented $\mathrm{Au}$ nanospheres and from the linked resonances enhancement from the underlying hybrid $\mathrm{Si}$ nanoweb. Previously, it has been shown that this hybrid Si nanostructure formed using the laserion plume formation mechanism is able to enhance the detection of chemical analytes, without the aid of SPR noble metal nanostructures; the Si nanoweb itself has an observable EF value on the order of $10^{6}$, which is comparable to many primarily noble metal based SERS enhancing nanomaterials ${ }^{[3-64]}$. The goal in this study was to determine the impact of combining the electromagnetic enhancement of the Au nanospheres and the linked resonances of the Si nanoweb structure has on the detection of chemical and bio-marker analytes in order evaluate this materials potential as a biocompatible SERS biochip sensing platform.

The observations of the Raman spectra nanostructures themselves have yielded results that are indicative of a hybridized enhancement from both the hybrid Si nanoweb and the Au nanospheres. Figure 3-4 shows the Raman spectra of the Si Wafer substrate, an Au ornamented substrate, a Si nanoweb substrate and an $\mathrm{Au}$ ornamented $\mathrm{Si}$ nanoweb substrate along with a simplified enhancement factor (EF) plot of the Raman response of the $520 \mathrm{~cm}^{-1}$ peak characteristic to $\mathrm{Si}$. These plots clearly show that the Si nanostructured surface has a substantial enhancement compared to the Si wafer substrate and that when ornamented with Au nanospheres the Si peak becomes muted on the ornamented nanoweb substrate. The Raman spectra and EF values in figure 3-4 show that indeed the ornamentation of the SERS active hybrid Si nanoweb with the Au nanospheres leads to a sizable increase in the nano-Raman activity of the material. It is theorized that the multiple SERS contributions lead to a highly sensitive platform for analyte sensing; the SPR from the Au nanospheres and the linked resonances from the Si nanoweb enhancement work harmoniously to increase the nano-Raman intensity.

NMNs are used a singular source for Raman enhancement contributor in most SERS and SERSrelated research ${ }^{[3-82-3-84]}$. NMNs have the unique characteristic of exhibiting the phenomena 
known as surface plasmon resonance (SPR) in the visible wavelength range, the same wavelength range used for Raman spectroscopy lasers. SPR occurs when incident photons cause a collective oscillation of electrons on the surface of a nanoparticle which in turn creates an electric field surrounding the nanoparticle ${ }^{[3-85]}$. If these nanoparticles are spaced at the correct distance from each other, the induced electric fields will overlap to create what is known as a "hotspot" ${ }^{\text {[3-86] }}$. These "hotspots" will scatter a larger percentage of photons which increases the observed Raman signal. A large variety of NMNs can be created using any number of fabrication techniques, each yielding an enhancement in Raman intensity. For example, researchers ${ }^{[3-87]}$ have fabricated $\mathrm{Au}$ nanostars with the ability to detect 1,4-aminothiophenol with an enhancement factor of $10^{7}$ while others $^{[3-88]}$ have observed Ag nanoparticles with the ability to detect 2-amniobiphenyl with an enhancement factor of $10^{7}$.

The underlying Si nanoweb material has been proven as a highly SERS active material without additional noble metal nanostructures. The Si nanoweb Raman active due to the SERS enhancing phenomena associated with semiconductors which are known as linked resonances ${ }^{[3-53]}$. These linked resonances include, exciton, molecular and charge-transfer resonance, which have previously been termed as the chemical enhancement contribution to SERS ${ }^{[3-53]}$. This chemical enhancement contribution is more often than not ignored when dealing with primarily noble metal because the electromagnetic contribution overwhelms any chemical contribution to enhancement ${ }^{[3-89]}$. However, with SERS-active semiconductor nanomaterials, the chemical contribution becomes the main contributor to enhancement and the electromagnetic contribution can be ignored. This is due to the relative photostability of semiconductors over the visible wavelength range with any induced SPR occurring in the infrared wavelength range. ${ }^{[3-90]}$ The chemical contribution to enhancement can be separated into several linked resonances but for this study a focus on the charge-transfer resonance for its relevance to the chemical and biosensing applications.

Charge-transfer resonance occurs when an analyte molecule, in close proximity to a nanostructured surface, is excited by a photon source. Although chemisorption is not required, the valence band (VB) and conduction band (CB) energy levels of the semiconductor substrate should be relatively comparable to the lowest unoccupied molecular orbital (LUMO) and highest occupied molecular orbital (HOMO) respectively. If these energy levels are within range of each other, and the incident 
light has enough energy, via a chemical bond, from either the semiconductor to the molecule or vice versa. The photons can excite an electron from the VB to the $\mathrm{CB}$ which due to the close proximity and comparable energy level to the LUMO, is transferred to the LUMO through resonant tunneling. This electron then decays back to the $\mathrm{CB}$, first transfers energy to a vibrational state of the molecule, which causes a Raman photon to be emitted from the molecule. Similarly, an electron can be excited by a photon from the HOMO energy level to the LUMO energy level and transferred to the $\mathrm{CB}$ of the semiconductor by resonant tunneling. The electron will then decay to the ground energy level of the molecule, transfer energy to the vibrational state of the molecule, and then the molecule will emit a Raman photon.

Figure 3-5 shows a schematic representation of the hotspot enhancement mechanism SPR metallic nanostructures and of the charge-transfer resonance of semiconductor nanostructures.

\section{Electromagnetic Enhancement (Metallic SPR)}

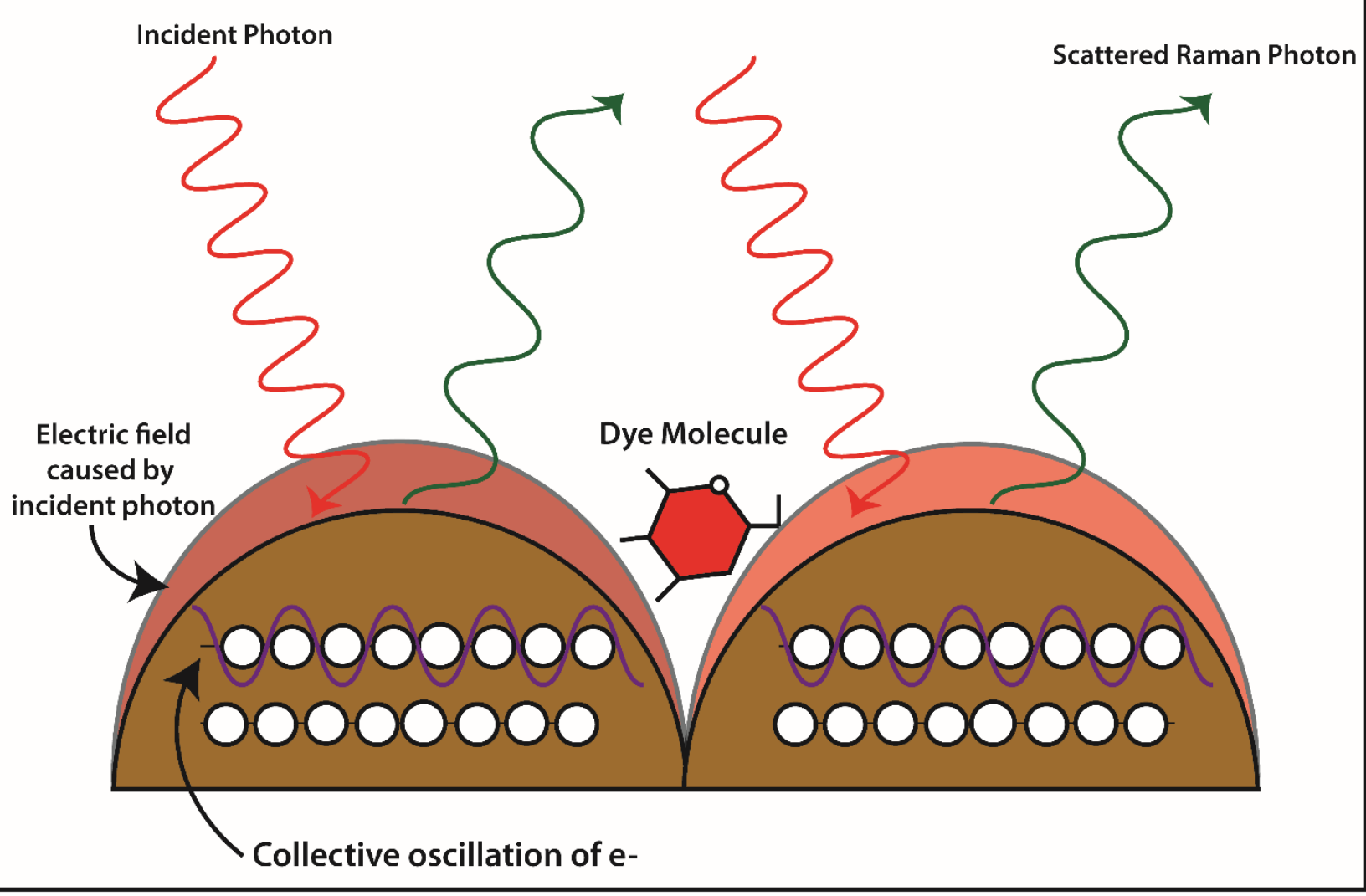




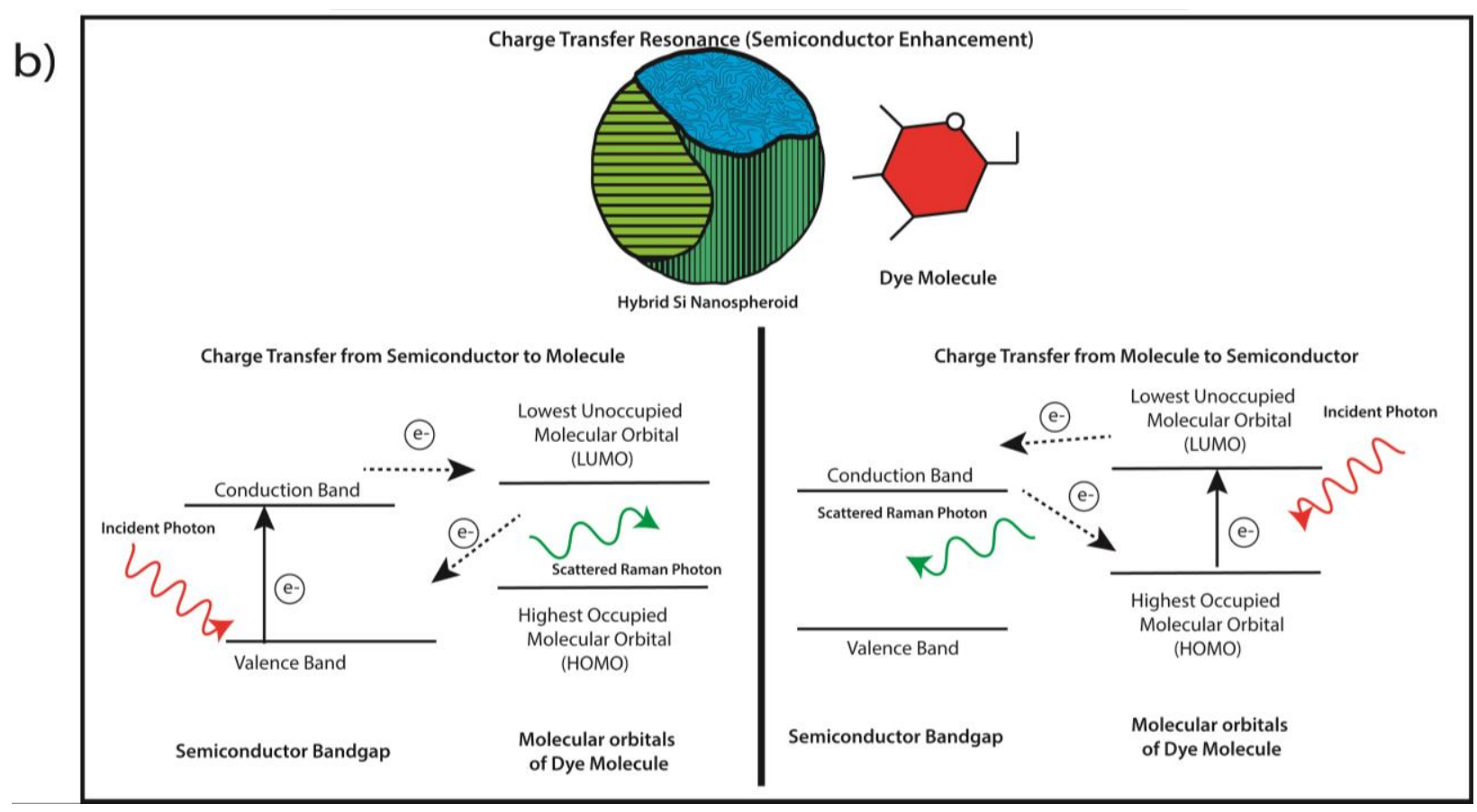

Figure 3-5: a) Schematic of SPR enhancement phenomenon and b) charge-transfer resonance principles

\subsubsection{SERS Biochip Enhancement Efficiency Analysis using CV dye}

The proposed primary use for this Au-NS ornamented hybrid Si nanoweb SERS biochip nanomaterial is for the detection of analytes at low concentration for chemical and biomolecule sensing applications. To determine this nanomaterials' capacity as a SERS biochip biosensing platform, it is required to observe if the ornamented nanoweb material is indeed able to sense the presence of a chemical analyte when coated onto its surface. To do this CV dye is coated on to the various biochip nanoweb structures that have been generated and taken the resultant Raman spectra. CV is chosen as a test analyte due to its high Raman cross-section, which enable for an easy evaluation of the sensing capabilities of the nanoweb structures. The level of enhancement generated by the nanoweb structures is quantified by calculating the enhancement factor (EF) value. This quantity is a ratio that compares the intensity at a characteristic peak value on the Raman spectra of the CV dye coated on the Au-NS ornamented Si-wafer substrate to the Au-NS 
ornamented hybrid Si nanoweb biochips, normalized by the number of analyte molecules on each respective substrates surface; Equation 3-1 is the enhancement factor ratio.

$$
E F=\frac{I_{N S} / N_{\text {surf }}}{I_{\text {sub }} / N_{\text {bulk }}} \quad \text { (Equation 3-1) }
$$

Where $I_{N S}$ and $I_{s u b}$ is the intensity of the Raman signal at the characteristic peak of the applied molecule, from the nanoweb structures and from the substrate respectively, $N_{\text {surf }}$ is the number of molecules contributing to the Raman signal from the nanoweb structure and $N_{b u l k}$ is the number of molecules contributing to the Raman signal on the substrate, as illustrated in Figure 3-6. This same formula was used in previous work ${ }^{[3-64]}$ to determine EF values; however some modifications have been made to increase the accuracy of the associated calculations.
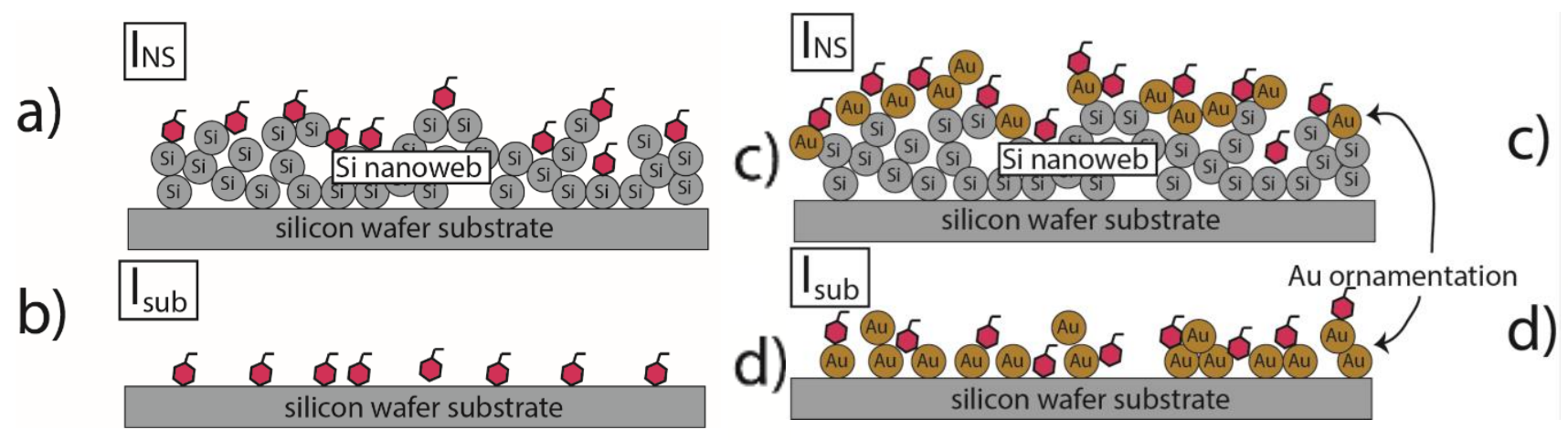

Figure 3-6: Schematic of sources of analyte spectra on a) Si nanoweb structures (top), on b) Si substrate (bottom), c) Au nanoweb structures (top) and on d) Au ornamented on Si substrate (bottom)

There are some approximations made when determining the number of molecules on the surface of each substrate, such as the surface area and material density due to the difficulty of experimentally obtaining these values for these nanoweb structures. To approximate the number of molecules that contribute to the Raman signal from the biochip nanoweb structure, it is necessary to consider how many molecules adsorb onto the surface of the nanoweb; due the difficulty of measuring this directly from the material that is formed, an assumed value determined by a literature survey of nanostructures of similar size and morphology is used. The value assumed is $7.8 \mathrm{~m}^{2} / \mathrm{g}$ obtained from the work by Cho et.al ${ }^{[3-91]}$ on porous silicon nanofibres. For both $N_{b u l k}$ and $N_{\text {surf }}$ it is assumed that only the molecules present within the interaction volume of the Raman 
laser will contribute to the signal. As such it is assumed that this interaction volume is a hemisphere with a diameter equal to the spot size of the Raman laser, $19.154 \mu \mathrm{m}$. In this equation, $N_{\text {bulk }}=$ $\pi r^{2} h c N_{A}$ where $r$ is the radius of the Raman laser spot size, $h$ is the penetration depth of the Raman laser, $c$ is the concentration of the analyte and $N_{A}$ is Avogadro's constant. Also in this equation, $N_{\text {surf }}=S A_{\text {eff }}\left(c_{a d s} N_{A} \frac{1000 L}{1 m^{3}}\right)^{2 / 3}$ where $S A_{\text {eff }}$ is the effective surface area of nanostructures within the Raman laser interaction volume, $c_{a d s}$ is the surface adsorption of analyte and $N_{A}$ is Avogadro's constant.

Figure 3-7 shows a schematic of this experimental study, the Raman spectra of the hybrid $\mathrm{Si}$ nanoweb biochip structures with Au-NP ornamentation coated with $\mathrm{CV}$ dye and the associated $\mathrm{EF}$ values as a function of plume energy for each of the biochip nanoweb structures.

a)

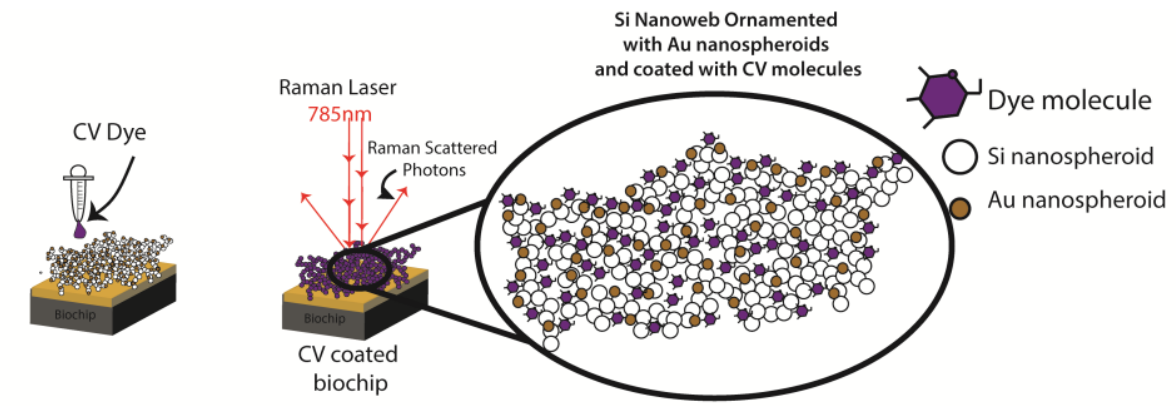

b)

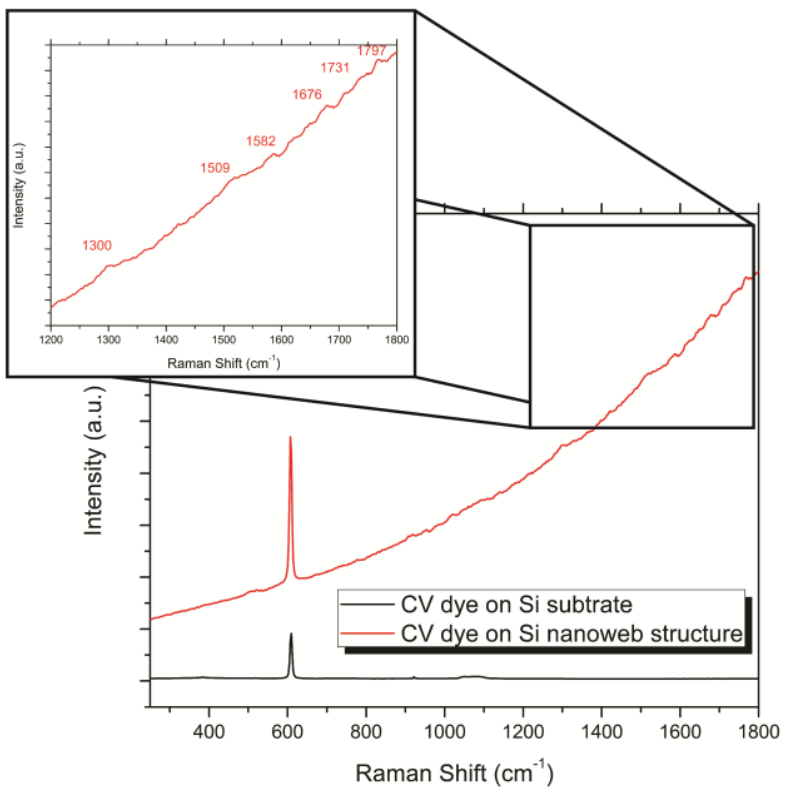

c)

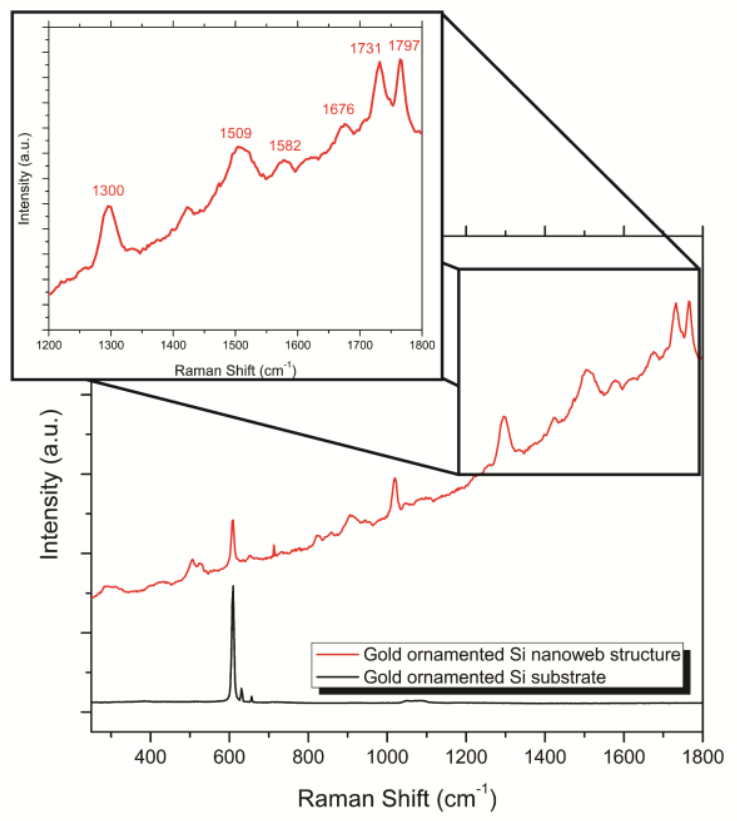




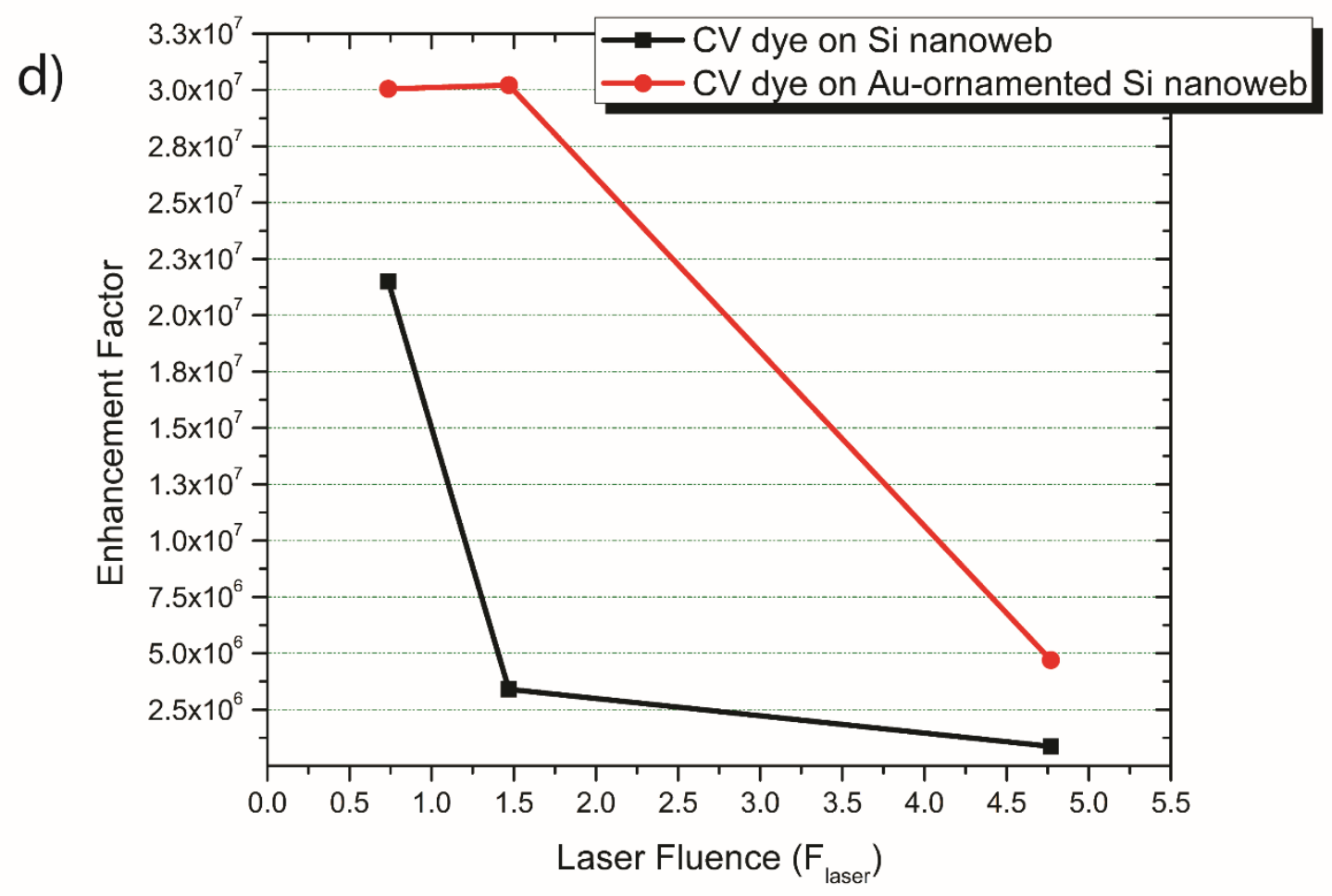

Figure 3-7: a) Schematic of experimental study of CV chemical analyte on ornamented hybrid Si nanoweb biochip structure with b) Raman spectra of CV on Si nanoweb structure and c) ornamented Si nanostructure, and d) associated EF values

These spectra show a clear enhancement of the detection of the CV dye on the biochip nanoweb surface and a correlation between the nanoweb morphology/composition and the level of enhancement.

As a proof-of-concept, these results give definitive results regarding the enhancement of a chemical dye on the silicon nanoweb structures and the same dye on gold nanosphere ornamented silicon biochip nanowebs. The Raman spectra show evidently that the Si nanowebs are able to enhance the detection of $\mathrm{CV}$ dye with a maximum calculated $\mathrm{EF}$ value of $2.2 \times 10^{7}$. The Aunanosphere ornamented Si biochip nanowebs however, show a substantial enhancement compared to the base Si nanoweb with a maximum EF of 3.0x10 ${ }^{7}$. These results demonstrate that the CV dye 
signal is boosted by the presence of the Au-nanospheres on the nanoweb surface, and thus the two enhancement mechanisms, the charge transfer mechanism and the electromagnetic SPR mechanism are both working congruently to enrich the dye signal. This material is unique because while others are able to observe high EF values with only contributions from a NMN, the nanomaterial presented in this study has a synchronous enhancement from a NMN and a Raman sensitive semiconductor nanostructure.

\subsubsection{Analysis for Application of SERS biochip enhancement using bio-label analyte (R6G)}

Sensing of bio-label molecules is of vital importance for the detection of labeled-DNA molecules. A majority of labelled DNA molecules have R6G molecules attached due to the high Raman crosssection of this molecule. This R6G molecule when attached to a DNA molecule becomes an indirect method of sensing the DNA molecules attached to the substrate surface; if a more intense R6G spectra is observed then is can be assumed that the substrate is able to enhance the detection of the attached DNA molecules ${ }^{[3-92]}$. In this study the enhancement efficiency of the Au-NP ornamented hybrid Si nanoweb biochip structure for the detection of the R6G molecule is determined, in order to evaluate if this material can be used as a DNA sensing biochip substrate. Using the nanoweb substrate with optimal laser-ion plume environment and Au-NS ornamentation conditions as the substrate for this experiment set, R6G molecule is applied to the biochips nanoweb structures at a set concentration $\left(10^{-6} \mathrm{M}\right)$ to test the enhancement for each biochip substrate. Figure 3-8 shows a schematic of this experiment, the observed Raman spectra and the calculated EF values. 

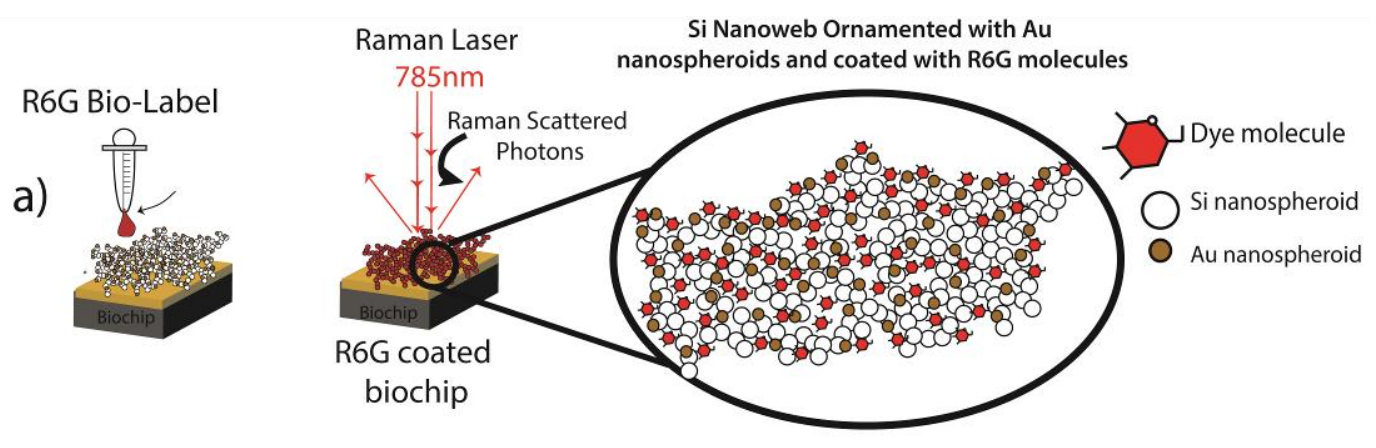

b)

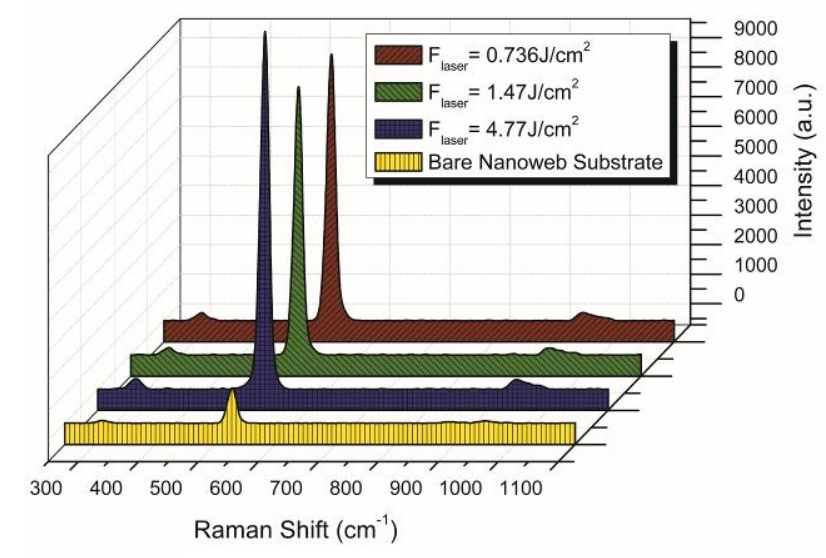

c)

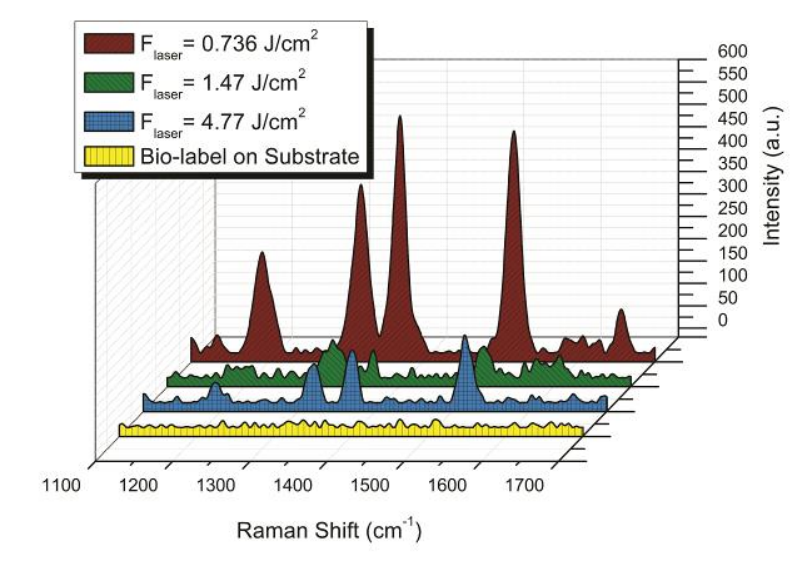

Figure 3-8: a) Diagram of ornamented hybrid Si nanoweb coated with R6G bio-label molecule and b) Raman spectra of each bare ornamented hybrid Si nanoweb and c) spectra of R6G biolabel on each nanoweb biochip structure

These spectra show a significant enhancement in the detection of the bio-label molecule. As seen in Figure 3-8, when the bio-label molecule is coated on the Au-NP ornamented Si wafer substrate, the characteristic peaks for the R6G molecule $\left(1320 \mathrm{~cm}^{-1}, 1370 \mathrm{~cm}^{-1}\right.$, and $\left.1576 \mathrm{~cm}^{-1}\right)$ are barely discernable from the background noise of the spectra. Conversely, when the bio-label molecule is applied to the surface of the Au-NP ornamented nanoweb biochip structures, the characteristic peaks not only become visible but the intensity of these peaks is increased considerably. As with the evaluation of the $\mathrm{EF}$ of the $\mathrm{CV}$ chemical dye, the degree of enhancement is evaluated by calculating the EF value using Equation 3-1. For the R6G at $10^{-6} \mathrm{M}$ concentration, the EF value 
that is observed is $1.4 \times 10^{7}$, which represents a vastly increased sensitivity to the bio-label molecule compared to the ornamented $\mathrm{Si}$ wafer substrate and is highly competitive with other noble metal enhancing materials.

\subsubsection{Influence of Ornamentation time on biochip enhancement efficiency}

One of the factors governing the enhancement of detection of the CV dye is the amount of AuNPs ornamenting the biochip nanoweb surfaces. As previously stated, the nanoweb biochip surfaces have been ornamented at three different ornamentation times to determine the role of the amount of Au-NPs has on the enhancement characteristics of the nanostructures. The spectra from this experiment will show the correlation between the amount of Au-nanospheres on the nanoweb surface and the contribution of electromagnetic enhancement from the au-nanospheres to the biolabel Raman signal.

Figure 3-9 is a representation of the Si-nanowebs ornamented for each length scale, the Raman spectra for each nanoweb structure for each time scale, and the enhancement factor calculated as a function of laser fluence. 
a)
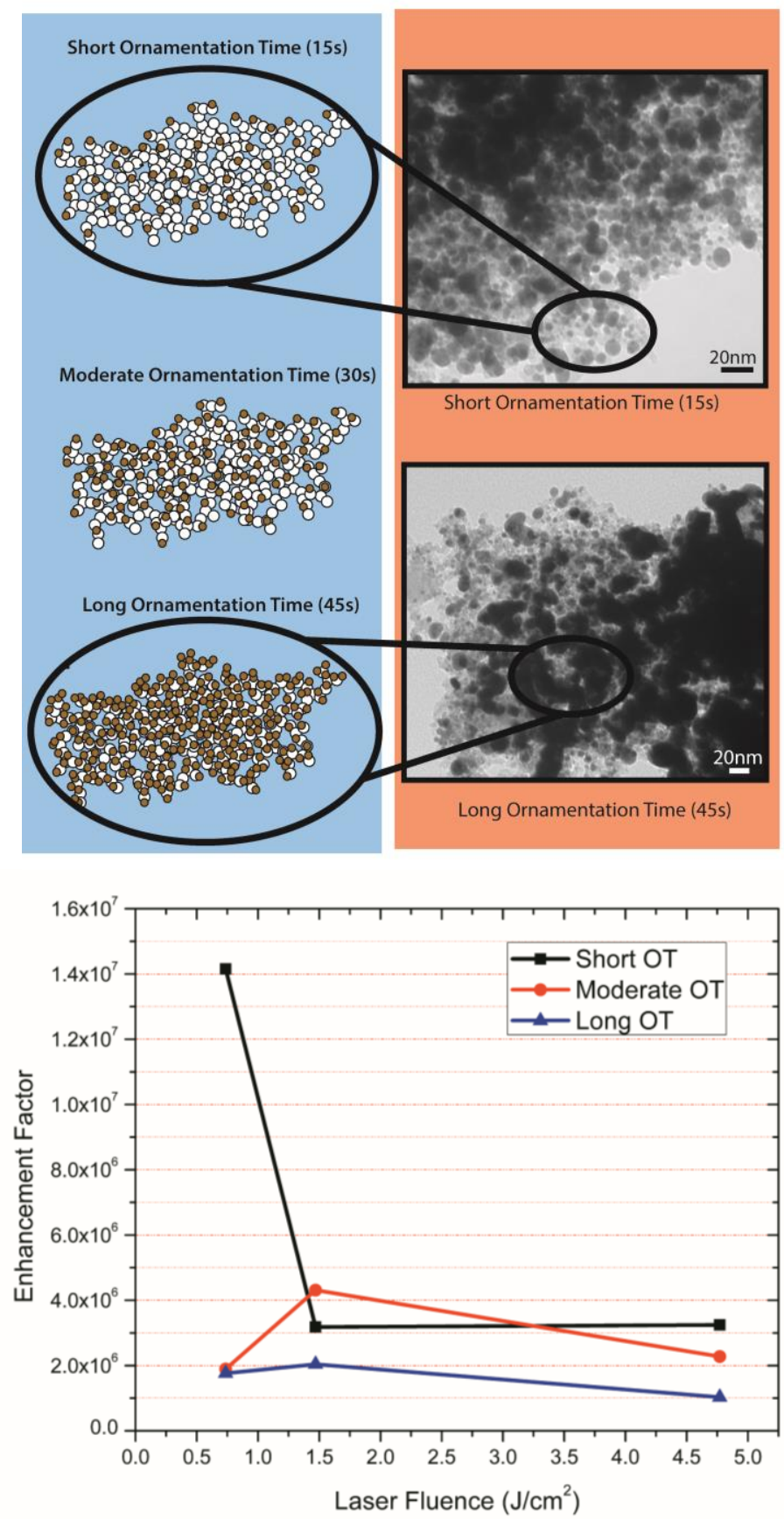

c)

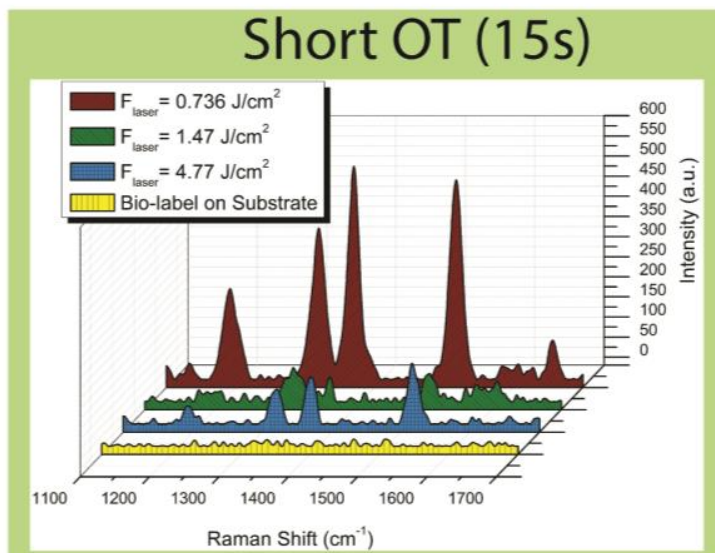

Moderate OT (30s)

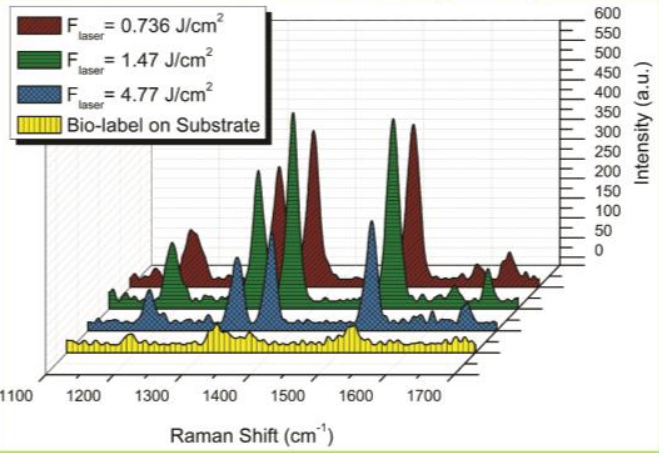

\section{Long OT (45s)}

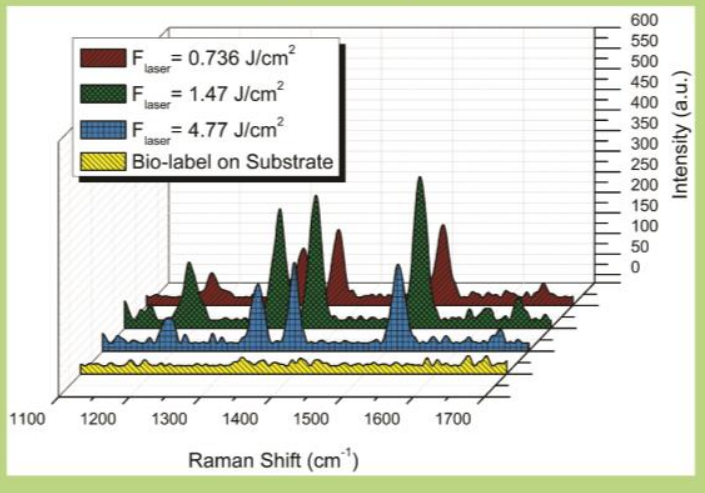

Figure 3-9: a) Representation of influence of OT on amount of Au nanospheres ornamented on

hybrid-Si nanoweb biochip structure, b) HRTEM images of short and long OT nanowebs, c)

Raman spectra of R6G on each nanoweb biochip structure and d) EF values associated with each biochip structure. 
The Raman spectra and EF show that the influence of ornamentation time and thus, the amount of $\mathrm{Au}$-nanospheres on the Si-nanoweb surface is not directly proportional to the enhancement of the R6G bio-label molecule, in fact as the amount of gold present on the nanoweb surface increases, the level of enhancement decreases considerably. The plot of EF values as a function of laser fluence in Figure 3-8 shows that a maximum EF $1.4 \times 10^{7}$ is observed at low fluence and $15 \mathrm{~s}$ ornamentation time and a minimum EF $3.2 \times 10^{6}$ is observed. A possible explanation for a smaller ornamentation time leading to a higher enhancement may be due to a masking effect from the $\mathrm{Au}$ nanospheres. While the Si nanoweb is being ornamented, Au nanospheres ejected from the target material bind to the surface of the interconnected nanospheroids and as time progresses, the Au nanospheres build up on top of each other resulting in a layer of Au the thickness and morphology of which is dependent on the ornamentation time. After a certain point, the Au-nanospheres on the surface create a type of nano-mask on top of the Si nanoweb, and the analyte molecules on the substrate surface will primarily be in close proximity to Au-nanospheres; this leads to the electromagnetic SPR phenomena being the major contributor to Raman signal from the biochip substrate.

In addition, it is possible that the thick Au nanosphere nano-mask present at longer ornamentation time is hindering the detection of Raman photons scattered by Si nanospheroids below the Au nanosphere layer; the bottom of the Au-nanosphere layer acts as a shield preventing these Raman photons from being detected by the Raman spectrometer.

At shorter ornamentation time, the analyte molecules will interact with both Au nanospheres and Si nanospheroids within the interaction volume of the Raman laser. This leads to a collective contribution to the enhancement of the analyte signal from both the electromagnetic mechanism and the charge-transfer mechanism. A precise tuning of both the formation of the hybrid Si nanoweb and the ornamentation with Au nanospheres is attained to create a "goldilocks" condition whereby the enhancement of the bio-label molecules exceeds that of other nanostructures that use either SPR or linked resonances as singular enhancement mechanisms. [3-93, 3-94]

In Figure 3-9 it is shown that for a particular ornamentation time (OT), different laser fluencies will have varied EF values; for instance at short OT the EF value reaches $1.4 \times 10^{7}$ at $0.736 \mathrm{~J} / \mathrm{cm}^{2}$ and at $4.77 \mathrm{~J} / \mathrm{cm}^{2}$ the EF value reaches only $3.2 \times 10^{6}$. This phenomenon arises due to mechanism that forms the underlying hybrid Si nanoweb structure; the laser-ion plume formation mechanism 
is able to create a nanoweb structure comprised of various $\mathrm{Si} / \mathrm{SiO}_{2}$ phases and varying physical morphologies of the individual hybrid Si nanospheroids that comprise the nanoweb structure. As such, the interaction between the hybrid $\mathrm{Si}$ nanospheroids, the analyte molecule, the $\mathrm{Au}$ nanospheres and the Raman laser photons will be dependent on the material chemistry and physical morphology of the $\mathrm{Si}$ nanoweb biochip structure. This masking and shielding effect is demonstrated in Figure 3-10.
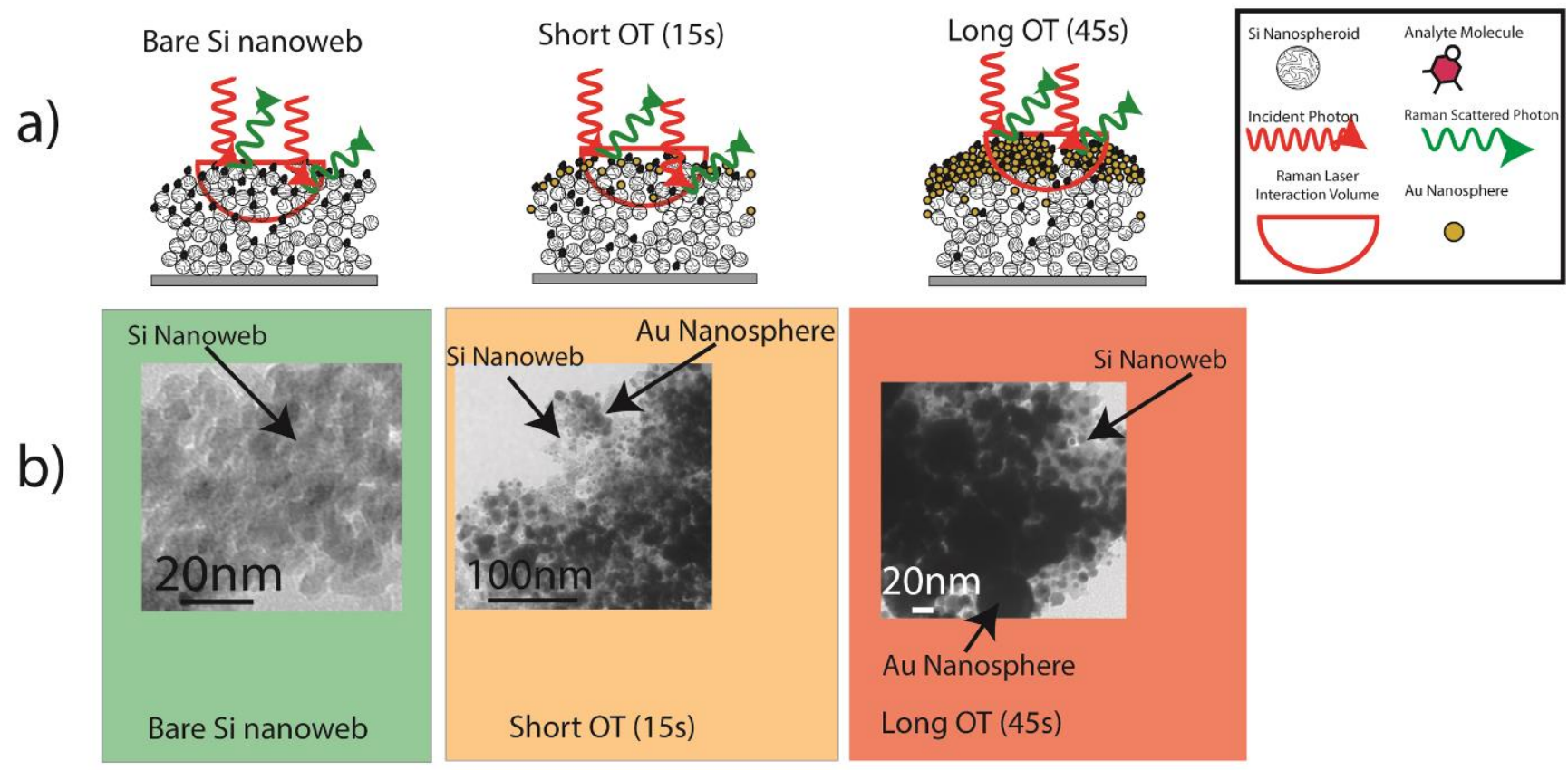

Figure 3-10: a) Schematic showing how OT time causes nano-masking and nano-shielding with b) HRTEM images of bare, short OT and long OT nanoweb structures.

The amount of gold present at each OT are characterized using XPS spectra in Figure 3-10 and it is observed that at longer OT the amount of gold present is $54 \%$ while at moderate OT the amount decreases to $43 \%$ and at short OT the Au amount decreases to $17 \%$. This nano-masking effect can also be observed in Figure 3-9 for the moderate OT, but becomes diminished at short OT.

The evidence that supports this nano-masking and nano-shielding effect can be seen in HRTEM images and XPS elemental analysis for each nanoweb biochip structure. Figure 3-11 shows the XPS spectra of three identical nanoweb biochip structures each with different OT, the elemental 
analysis of $\mathrm{Si}, \mathrm{O}$ and $\mathrm{Au}$ present in each nanoweb and HRTEM images corresponding to each nanoweb. The XPS spectra and elemental analysis plainly show that as OT increases the amount of $\mathrm{Au}$ on the surface increases from $~ 17 \%$ at short OT to $\sim 43 \%$ at moderate OT to $54 \%$ at long OT, while the presence of $\mathrm{Si} / \mathrm{SiO}_{2}$ decreases as OT increases. The HRTEM images also support this observation, in Figure 3-10 at short OT the Au layer on the Si nanoweb is relatively light compared to the moderate OT image and the short OT image shows a thick layer of Au on the Si nanoweb, so much so that the Si nanoweb is difficult to observe.

As OT increases more and more Au nanospheres occupy the interaction volume of the XPS spectrometer, to a point where the majority of matter that the XPS interacts with is the Au nanospheres. This same principle applies to the space occupied by Au within the Raman laser interaction volume; at long OT, most of the interaction volume will be Au nanospheres and as such the bio-label molecules will mostly interact with $\mathrm{Au}$ nanospheres and less with hybrid $\mathrm{Si}$ nanospheroids which then lead to the major contributor to SERS being an electromagnetic component not a charge transfer component. As our observation and calculation of the EF for the bio-label molecule indicates, the electromagnetic component is less effective as a primary contributor to the SERS enhancement and becomes more effective when contributing harmoniously with the charge transfer component from the hybrid Si nanoweb biochip structure. 

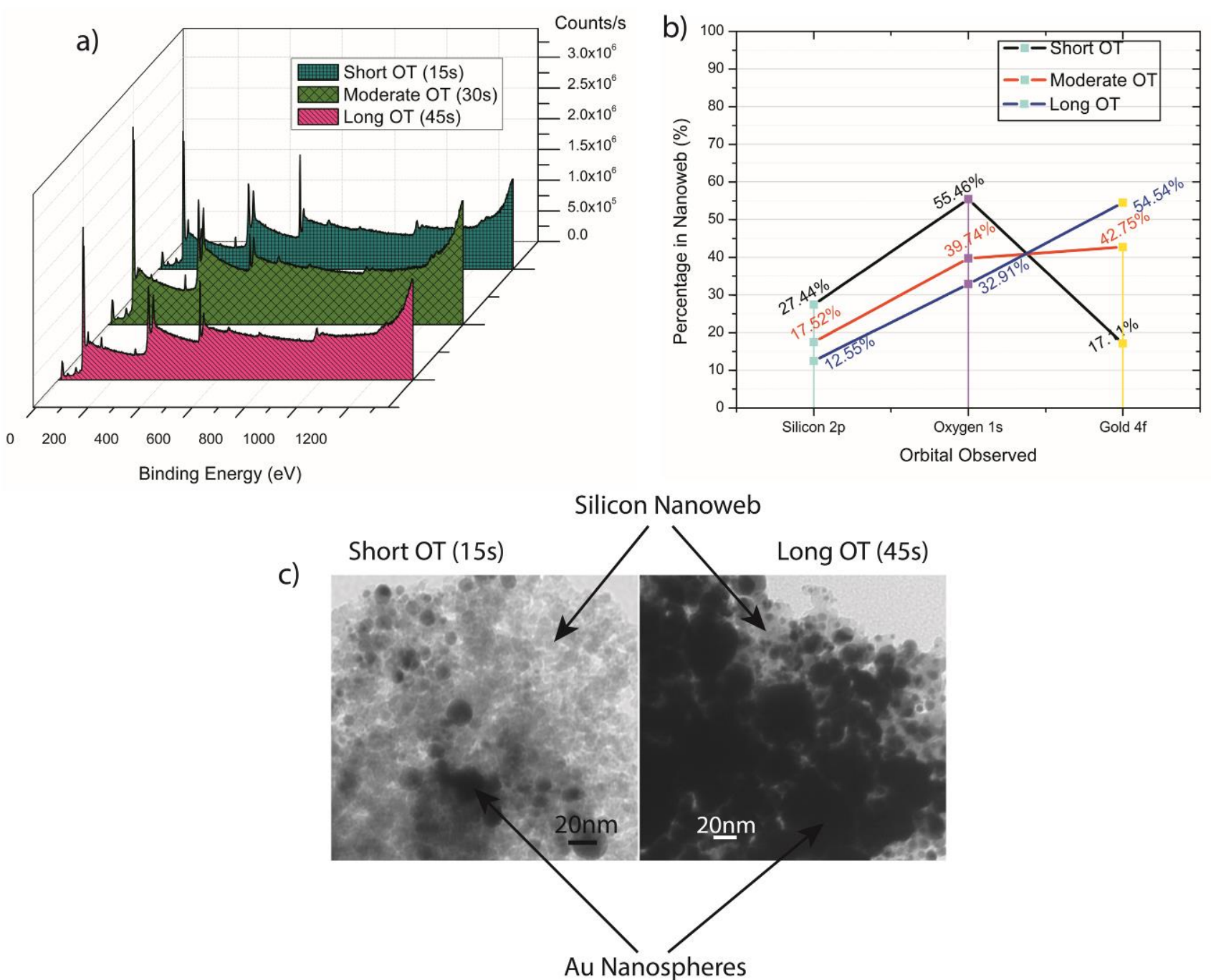

Figure 3-11: a) XPS spectra for short, moderate and long OT nanoweb biochip structures with b) percentage values of $\mathrm{Si}, \mathrm{O}$ and $\mathrm{Au}$ at each OT and c) HRTEM images of short OT and long OT nanoweb biochip structures. 


\subsection{Summary}

In this study, it is shown that the unique Raman active hybrid amorphous/crystalline Si nanoweb material has the potential to further stimulate the detection of chemical and biochemical analytes using noble metal nanosphere ornamentation of the outer surface of the nanostructure as a biochip biosensor device. With both the semiconductor based linked resonance enhancement mechanism and the noble metal based SPR enhancement mechanism each working harmoniously, a level of Raman enhancement not seen with other similar nanostructured materials is observed. By exerting the level of control over the laser-ion plume formation mechanism a precise tuning of the formation of the Raman active base hybrid Si nanoweb structures, whose enhancement capabilities has been augmented with the ornamentation of Au nanospheroids onto the nanoweb outer surfaces; with this amplified Raman-active nanomaterial, an EF value on the order of $10^{7}$ for $\mathrm{CV}$ dye at $10^{-6} \mathrm{M}$ is observed. It is also shown that this ornamented hybrid nanoweb material has the potential to be a highly sensitive bio-sensing substrate due to the $10^{7} \mathrm{EF}$ values that has been observed for the biolabel molecule R6G at $10^{-6} \mathrm{M}$. These results prove that this new hybrid material has substantial potential for highly sensitive SERS biosensor applications and can be easily integrated into existing fabrication methods in order to form SERS biochip sensor substrates. 


\section{Chapter 4}

\section{SERS detection of protein biomolecule on polymorphic Si interconnected nanospheroid nanocore network platform with secondary noble metal nanosatellite boosting mechanism}

Under peer review in RCS Advances, 2017

In this chapter, the development of a polymorphic biosensitive Si nanocore superstructure as a high-performance SERS biosensing platform is reported. The polymorphic Si nanostructure in this study is created through ultrafast pulse laser ion-plume (UPLIP) formation and an observed enhanced detection of L-glutathione (GSH). The distinctive polymorphic nanomaterial chemistry of the interconnected network of $\mathrm{Si}$ nanocores, and the nanonetwork architecture of this nanostructure acts as a primary enhancement booster. With the addition of Au/AuPd nanosatellites onto the surface of the polymorphic Si nanocore structure, a significant secondary boost in GSH enhancement is observed. The addition noble metal nanostructures results in multi-source SERS enhancement that combines linked resonance enhancement and SPR mechanisms that both contribute to the detection and boosting of the biomolecule analyte signal. With this polymorphic $\mathrm{Si}$ primary booster and noble metal nanosatellite secondary booster, the viability of $\mathrm{Si}$ nanostructures for SERS biosensing applications is demonstrated.

\subsection{Introduction}

Of the available techniques used for biosensing with nanomaterials, which include electrochemical ${ }^{[4-1]}$, pyroelectric ${ }^{[4-2]}$ and optical techniques ${ }^{[4-3]}$, SERS-based biomolecule detection offers distinctive advantages compared to the other methods including ultrahigh sensitivity ${ }^{[4-4]}$ and high specificity $^{[4-5]}$. For biomolecule detection with SERS, most sensing platforms utilize the surface plasmon resonance (SPR) mechanism characteristic of noble metal nanostructures (NMNs) as the primary source for SERS enhancement ${ }^{[4-6-4-8]}$.

SERS active NMNs are used for the detection of biomolecule analytes such as glucose ${ }^{[4-9]}$ and nucleic acids ${ }^{[4-10]}$. These SERS active NMNs are predominantly generated through the deposition of noble metal nanoparticles onto the surface of in-active nanostructures, which serves as a carrier 
platform for the SERS active noble metal nanoparticles. ${ }^{[4-11,4-12]}$ These quiescent scaffolding nanostructures are commonly semiconductor nanomaterials, such as $\mathrm{Si}$ nanowires ${ }^{[4-13]}$, or $\mathrm{Si}$ nanostructures created by nanolithography ${ }^{[4-14]}$ or chemical synthesis. ${ }^{[4-15,4-16]}$ Currently, the detection of biomolecules with SERS is focused on mainly NMNs as a SERS activator while the underlying nanostructure has no reported SERS activity, to our knowledge.

However, there is a significant lack of research regarding the use of SERS biosensitive semiconductor nanostructures for bio-detection applications despite recent observations of semiconductor nanostructures having significant SERS activity. ${ }^{[4-17,4-18]}$ Furthermore, very little research has been conducted to establish $\mathrm{Si}$ as SERS biosensing platform due to the SERS inactive nature of traditionally fabricated Si nanostructures. The work by Lombardi and Birke ${ }^{[4-19]}$ define the fundamental principles of SERS activity of semiconductor nanomaterials. It has been recognized that several linked resonance mechanisms, each contribute to an observed semiconductor SERS enhancement ${ }^{[4-19]}$; these mechanisms are plasmon resonance, exciton resonance and charge transfer resonance. These mechanisms work in tandem to increase an analyte signal, and a number of semiconductor materials have been shown to exhibit SERS enhancements with these linked resonances. Several nanomaterials, including $\mathrm{TiO}_{2},{ }^{[4-20]} \mathrm{ZnS}^{[4-21]}$ and $\mathrm{CdSe}^{[4-22]}$ have been shown to have significant sensing performance for chemical analytes. These nanostructures represent significant strides for semiconductor SERS chemical sensing, however these nanostructures are mostly limited to nanostructures such as nanoparticles ${ }^{[4-18]}$ or nanorods..$^{[4-}$ ${ }^{23]}$ Of the 3D SERS active nanostructures that include semiconductor nanostructures, nearly all of them rely these nanomaterials as a scaffolding or template for NMNs, which is reported as the sole SERS enhancement source. ${ }^{[4-24]}$ As such, the development of 3D semiconductor nanostructures as primary or co-contributors to the SERS enhancement of analyte detection is quite limited, especially for biomolecule sensing applications. Previous studies of the SERS enhancing characteristics of 3D silicon nanoweb structures have proven these nanostructures viable for chemical sensing applications ${ }^{[4-25,4-26]}$ and have established these as highly active SERS nanostructures. While these semiconductors have received some research and development, to our knowledge the biosensing applications of semiconductor-based biosensing nanomaterials and specifically, self-assembled 3D Si nanostructures have yet to be explored. 
In this study it is introduced for the first time, to our knowledge, the concept of a Si nanocore enhancement mechanism for a boosted enhancement of biomolecule detection. In addition, an introduction of the concept of a multi-source boosting mechanism for a further increase in enhancement of a biomolecule signal using a secondary SERS enhancement boost with the use of noble metal nanosatellites. The underlying principle is that, both a bio-SERS active $\mathrm{Si}$ semiconductor nanostructure platform and noble metal nanosatellites provide a primary and secondary boosting effect, respectively, on the biomolecule signal through independent enhancement mechanisms.

The SERS active Si nanocores act as a primary booster to the detection of a biomolecule analyte. The Si nanocore superstructure is comprised of a nanonetwork of fused Si nanospheroids that provide an enhancement of SERS biomolecule signal through linked resonance enhancement mechanism. The unique polymorphic structure of the Si nanocores and the self-assembled 3D nanonetwork morphology of the fused nanocores provides the SERS bio-activity not observed in traditionally fabricated $\mathrm{Si}$ nanostructures. The Au and AuPd nanosatellites act as a secondary boosting source for SERS biomolecule signal boosting by providing a SPR enhancement mechanism effect characteristic of NMNs. It is due to these primary, and secondary biomolecule signal boosting mechanisms working co-operatively, it is observed that the biomolecule detection is significantly enhanced. In addition, by modifying the characteristics of the Si nanocore superstructure and the nanosatellites, a balance between the two enhancement mechanisms can be achieved that leads to greatly enhanced biomolecule detection.

By using the laser-ion plume formation mechanism to form the interconnected hybrid $\mathrm{Si}$ nanospheroid nanocore superstructure, a SERS biomolecule active 3D platform is formed. For the deposition of $\mathrm{Au}$ nanosatellites or AuPd nanosatellites a physical ion deposition method is employed; this combination of nanomaterials leads to a dual-contribution to SERS biomolecule enhancement from linked resonance enhancement and SPR, for a large increase in the enhancement of biomolecular sensing. To establish the Si nanostructure as a SERS biosensing nanomaterial, this nanomaterial has been utilized for the detection of L-glutathione (GSH), a tripeptide biomarker present in living cells including cancer cells which indicates the amount of stress the cell is undergoing and as a result if the cell is dying. ${ }^{[4-27,4-28]}$ With the detection of this biomolecule, the goal is to define the role of highly SERS biosensitive polymorphic Si 
nanostructure in the field of SERS biosensing. An overview of this study is presented in Figure 41

a)

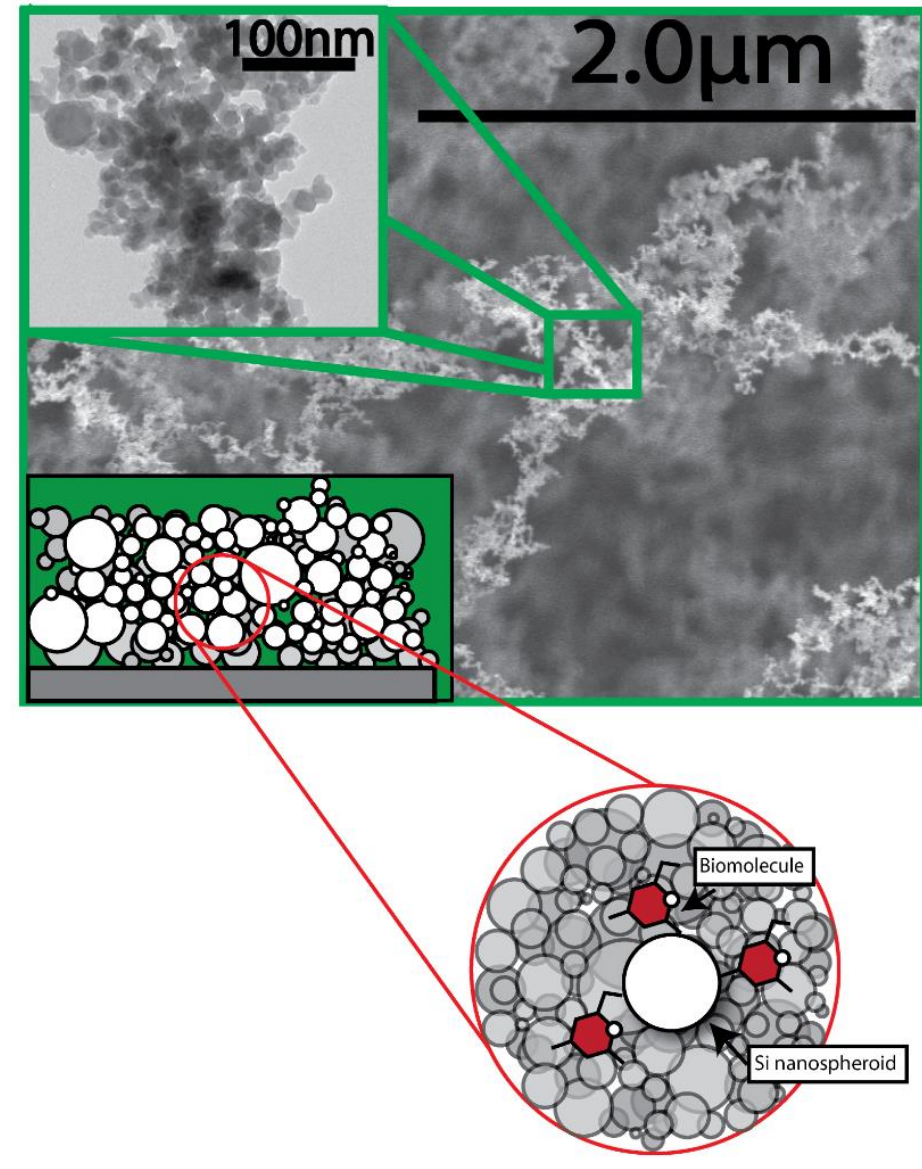

Raman spectra of Si nanocores @532nm
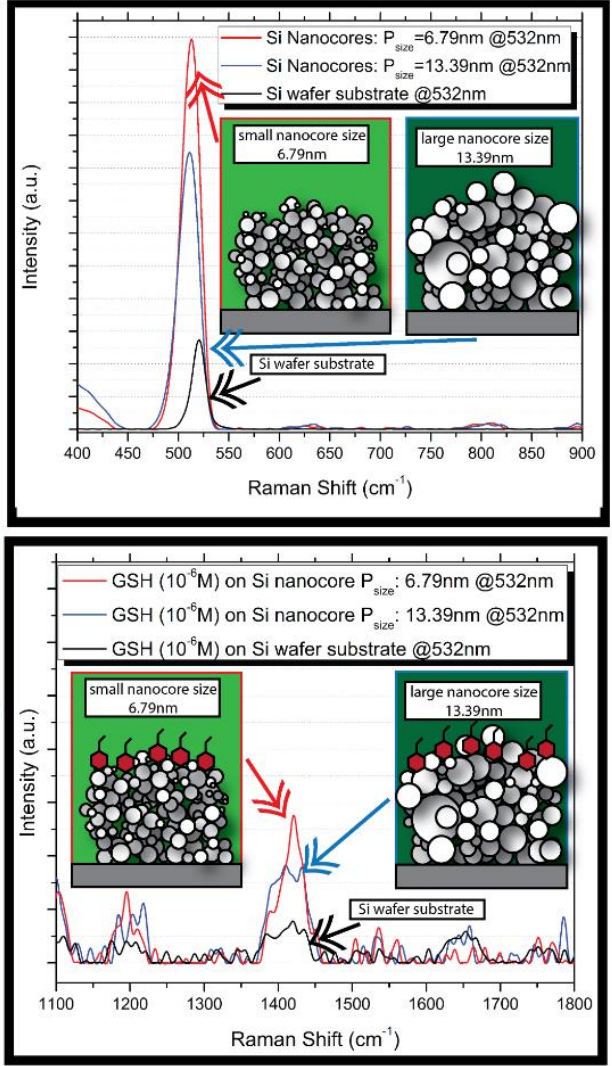

GSH on Si nanocores @532nm 
b)

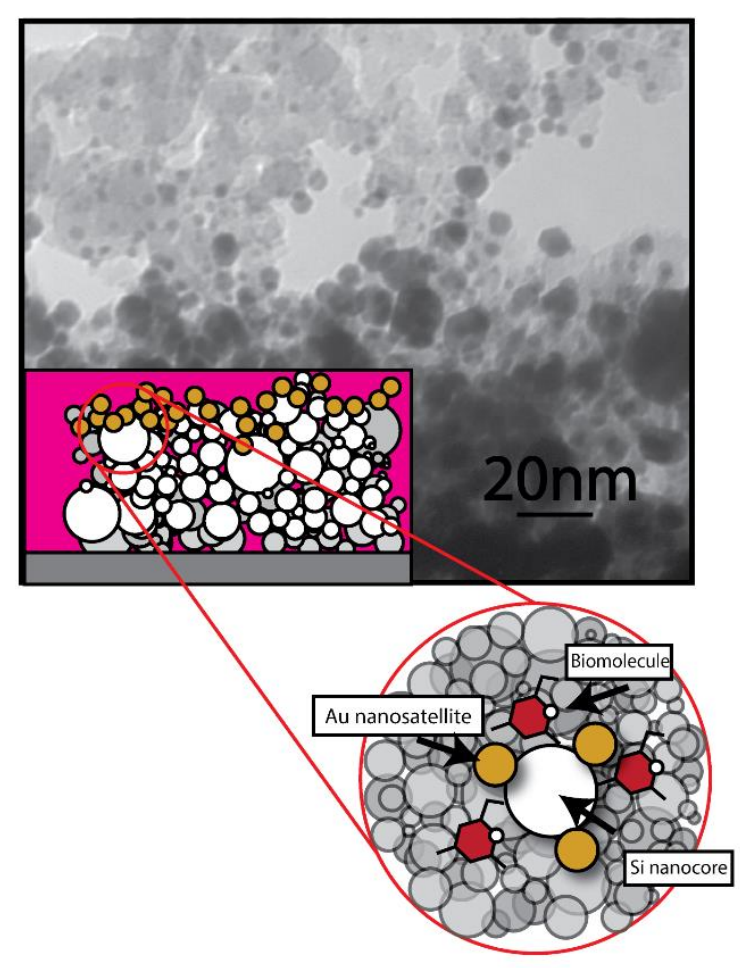

Raman Spectra of Si nanocores w/ Au nanosatellites @532nm
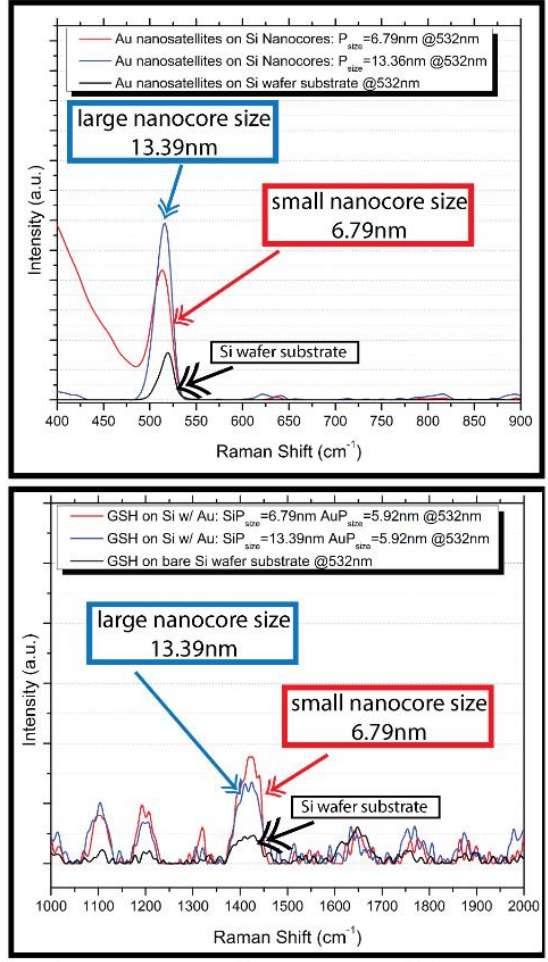

GSH on Si nanocores w/Au nanosatellites @532nm c)

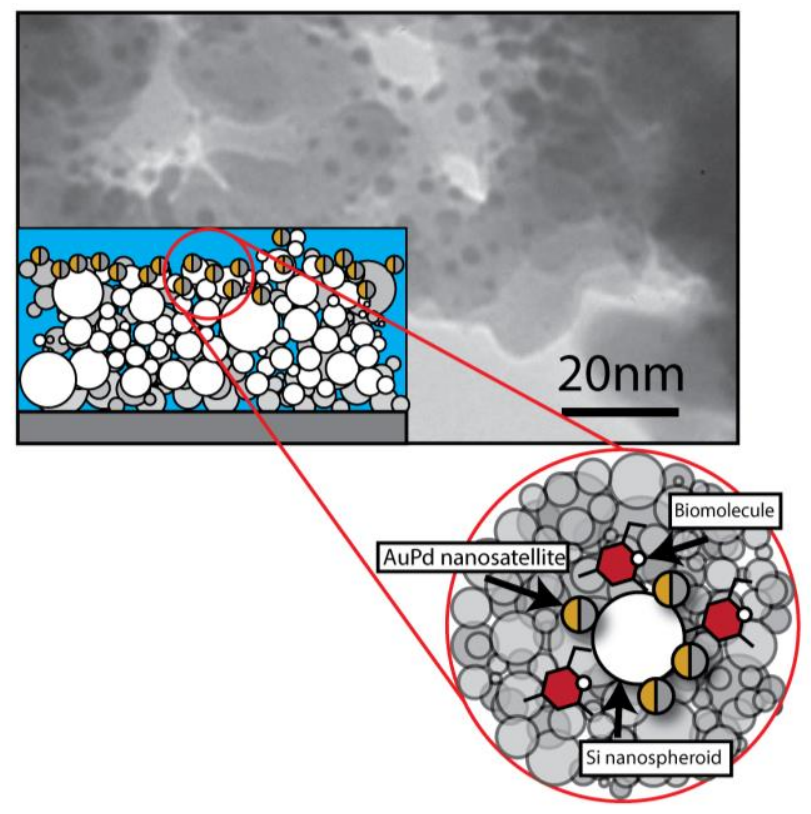

Raman Spectra Si nanocores w/ AuPd nanosatellites @532nm
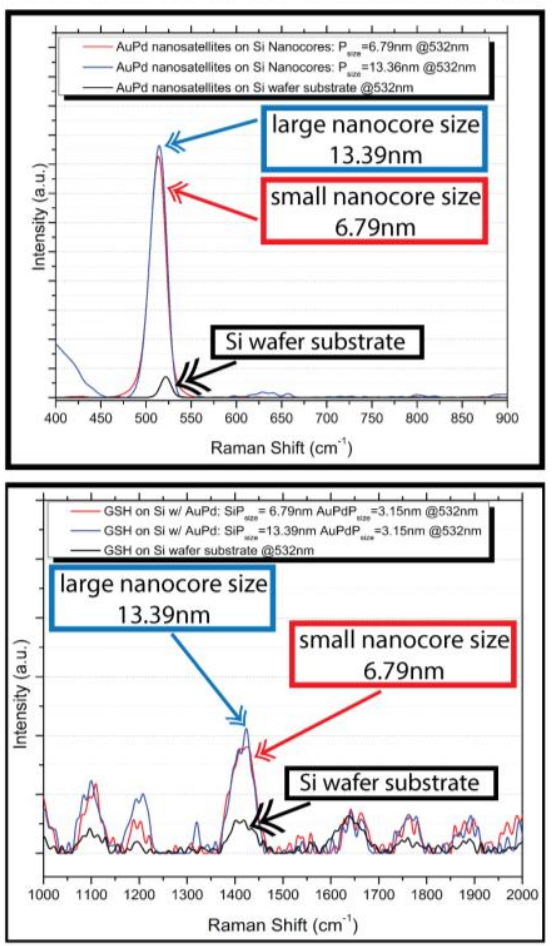

GSH on Si nanocores w AuPd nanosatellites @532nm 
Figure 4-1: Raman spectra with TEM and schematic diagrams of a) Si nanocore enhancement of $520 \mathrm{~cm}^{-1}$ peak at $532 \mathrm{~nm}$ with spectra of GSH on Si nanocores at 532nm b) Si nanocores with $\mathrm{Au}$ nanosatellites showing enhancement of $520 \mathrm{~cm}^{-1} \mathrm{Si}$ peak and spectra of GSH @ 523nm and c) Si nanocores with AuPd nanosatellites showing enhancement of $520 \mathrm{~cm}^{-1}$ Si peak and spectra of

GSH@523nm wavelength

\subsection{Experimental Method}

The formation of the polymorphic Si nanocore structures are generated using a Clark-MKR IMPLUSE pulsed Yb-doped fibre amplified femtosecond laser. This pulsed laser system initiates the ultrafast pulsed laser ion plume (UPLIP) required to form a nanocore structure. In this study, $0.02-\Omega \mathrm{cm}$ p-type silicon (100) wafer substrates were used to create the polymorphic $\mathrm{Si}$ nanostructures. To have full control over UPLIP fabrication process, in this experiment the laser wavelength $(1030 \mathrm{~nm})$, polarization (circular), dwell time $(0.5 \mathrm{~ms})$, pulse width (214fs) and the laser power $(16 \mathrm{~W})$ were kept static. The main UPLIP parameter varied in this study is the repetition rate of the laser, with which the nanospheroid formation conditions of the laser ion plume can be controlled. In order to create a consistent distribution of nanocore structures on the base $\mathrm{Si}$ wafer, the nanostructures were generated using a 300x300 point array with 25 micron point spacing; a piezo-driven raster was used to move the laser beam across the substrate surface using an array designed with EzCAD software.

To coat the surface of the polymorphic Si nanocore structures with nanosatellites a physical deposition method was used; a 99.9\% pure Au target and an AuPd target were bombarded with ion particles with an atmosphere of Argon gas to ionize the target. The ejected ions then physically deposit onto the surface of the nanocore structures for a set time. The deposition time, $15 \mathrm{~s}$ established in previous studies has been used, to increase maximize the SPR contribution from the nanosatellite structures.

For the acquisition of Raman spectra, a Bruker Optics SENTERRA Raman microscope was used using a 10x magnifying lens. Spectra of the Si nanocore nanostructures were obtained using 532nm wavelength with a 10s integration time and 3 iterations. To compare the polymorphic Si nanocores to the nanocores with $\mathrm{Au}$ or AuPd nanosatellites $5 \mathrm{~mW}$ power was used for 532nm Raman spectra. 
For the GSH spectra acquisition, $10 \mu \mathrm{L}$ of $10^{-6} \mathrm{M}$ concentration GSH was applied to each nanocore substrate and Raman spectra were obtained using the same parameters above.

To determine the morphology of the nanocore structures and to measure and calculate the nanospheroid/nanosatellite sizes, high resolution tunnelling electron microscopy was used. ImageJ software was used to manually measure and categorize the nanospheroid/nanosatellite sizes for each nanocore substrate.

XRD analysis using a Rigaku MiniFlex 600 was performed to determine the crystallographic make-up of the different structures used in this study. The $2 \Theta$ scan range from $15^{\circ}$ to $60^{\circ}$ was used to acquire the peaks associated with the Si nanocore structures.

\subsection{Results and Discussion}

The morphology of the Si nanostructures is influenced by the formation properties of the UPLIP process including the repetition rate of the laser pulses and the dwell time per ionization spot; although it is more informative to identify the nanocore structures by their physical properties. In terms of the UPLIP process, the nanocore structures are classified by the nanospheroid median diameter and the size distribution of nanocores throughout the network. The nanosatellite deposition process is kept consistent to maintain reliability between the Au and AuPd enhancement effect and the Si nanocore enhancement effects. XRD has been used to classify the material chemistry of each nanocore structure. Figure 4-2 shows the nanospheroid size and distribution curves as well as the XRD from the nanostructures. 

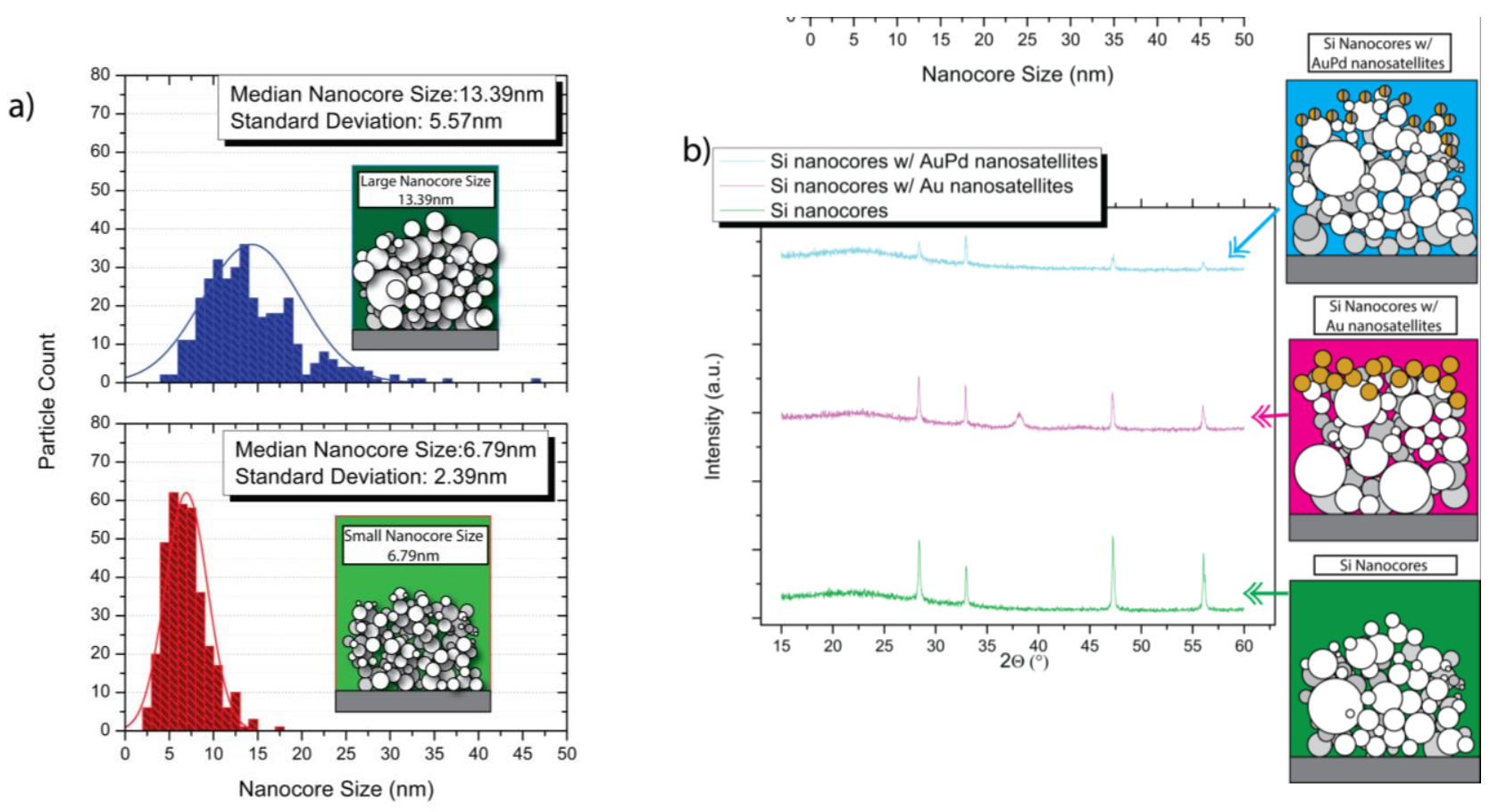

Figure 4-2: a) particle size distribution plots for small $(6.79 \mathrm{~nm})$ and large $(13.39 \mathrm{~nm}) \mathrm{Si}$ nanocores and b) XRD spectra of Si nanocore, Au nanosatellite deposited Si nanocore and AuPd nanosatellite deposited Si nanocore substrates.

Figure 4-3 shows the Raman spectra of the different polymorphic nanocore structures that have been created in this study based on their median particle size/size distribution and a plot of the EF of these nanostructures compared to the Si wafer substrate. In addition Figure 4-3 shows the Raman spectra and associated EF values of the Si nanocores with Au and AuPd nanosatellites for the $520 \mathrm{~cm}^{-1}$ Si peak. 

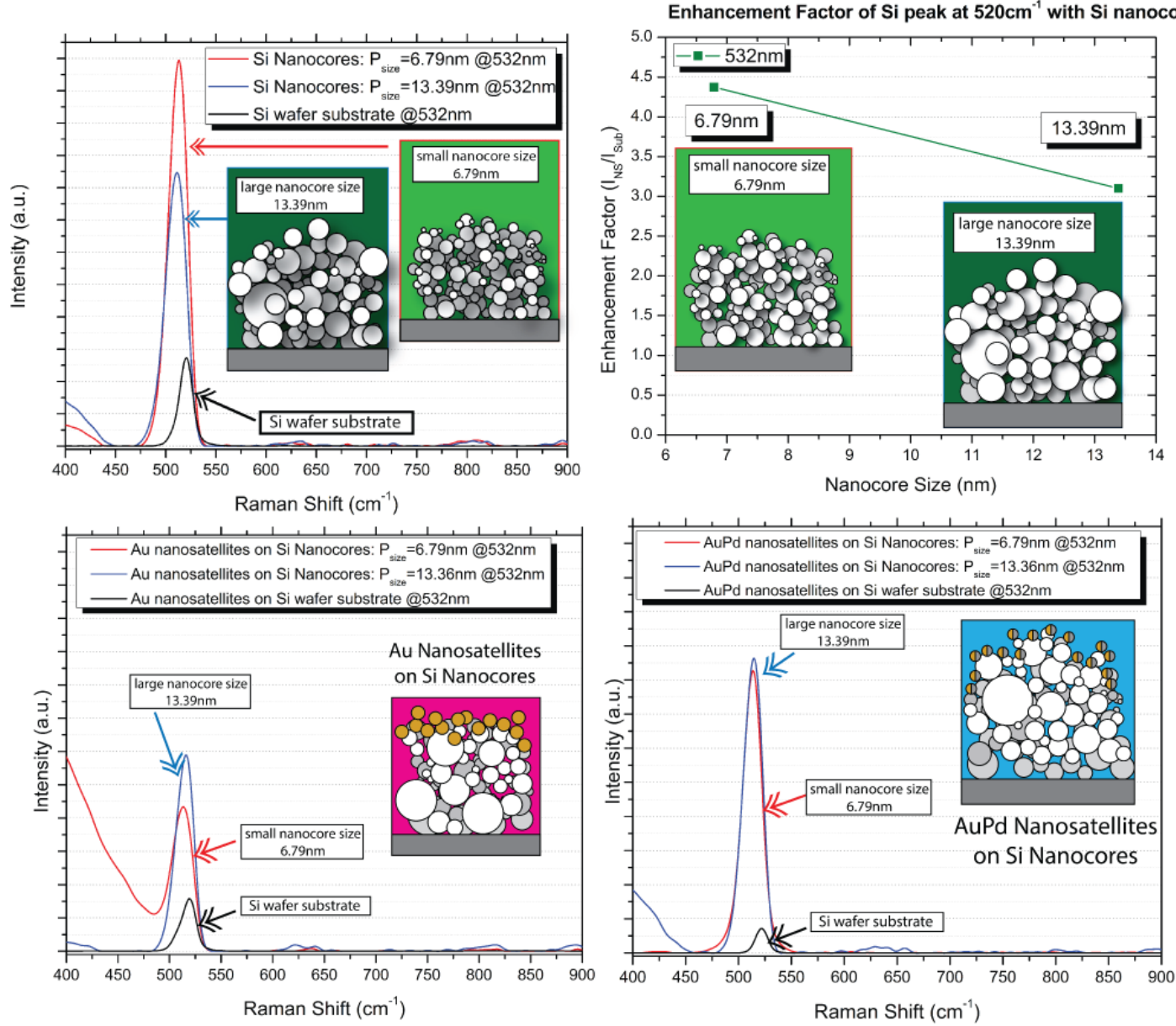

EF: All Subs @532nm, Si, AuSi, AuPdSi NSonly

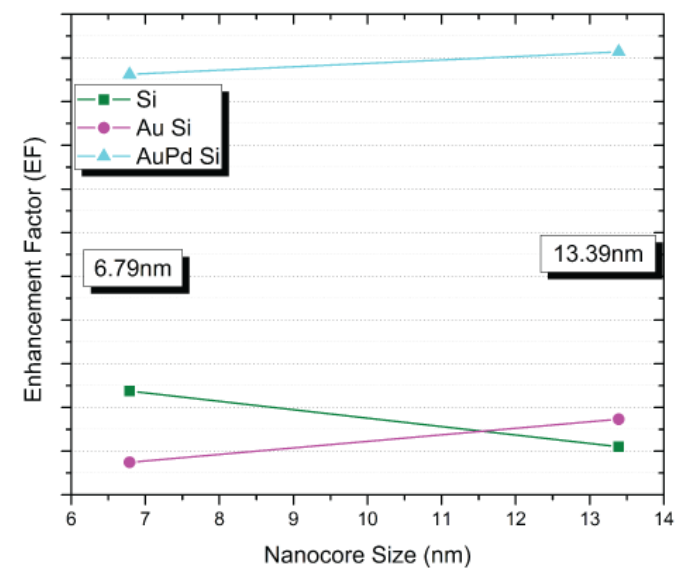

Figure 4-3: a) Kaman spectra showing peak intensity of $520 \mathrm{~cm}^{-1}$ peak tor $\mathrm{S} 1$ water substrate (black), large nanocore size (blue) and small nanocore size (red) and b) the EF value at $520 \mathrm{~cm}^{-1}$ of the small and large nanocore structures relative to the $\mathrm{Si}$ wafer substrate c) NS spectra for $\mathrm{Si}$ nanocores w/ Au nanosatellites, d) Si nanocores w/ AuPd nanosatellites and e) the EF of each substrate 
Figure 4-4 shows the Raman spectra of the GSH biomolecule at micromolar and concentration and the calculated EF values and the Raman spectra for the GSH on Au nanosatellite nanocores and AuPd nanosatellite nanocores with the corresponding GSH spectra for bare Si nanocore substrates and the EF values with the respective nanosatellite Si wafer substrates. The EF values for the GSH on the nanosatellite nanocore structures is calculated using the same equations and assumptions used in the Si nanocore structures experiment.

a)

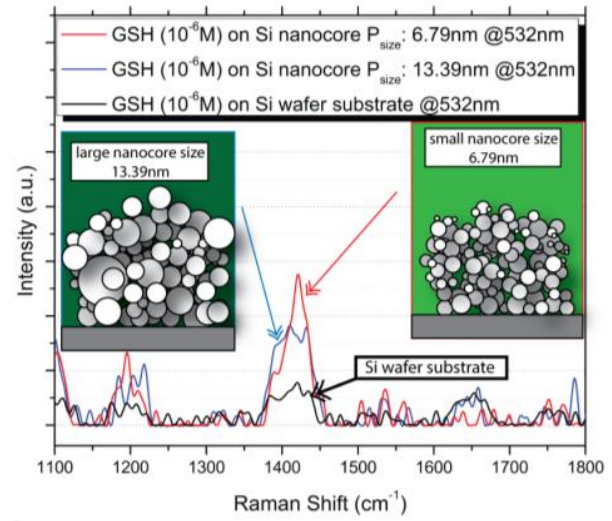

c)

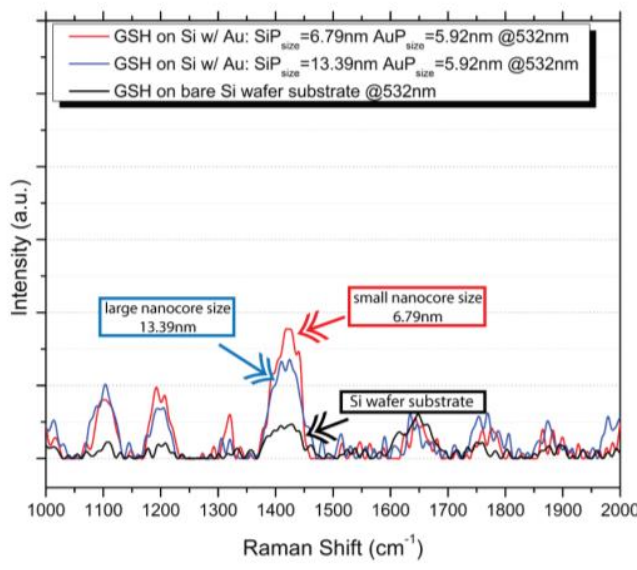

b)

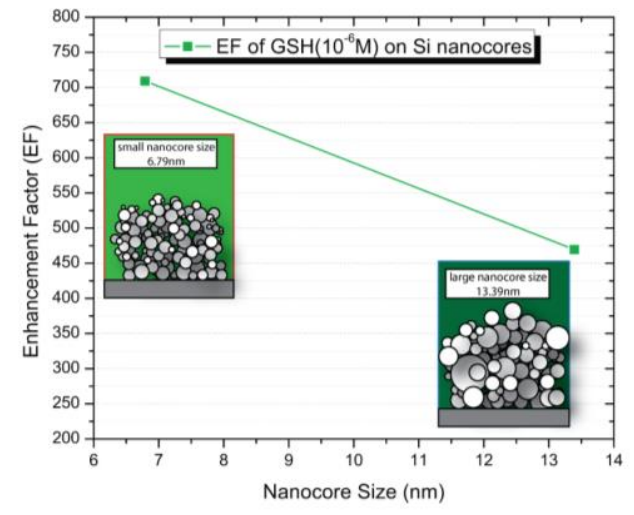

d)

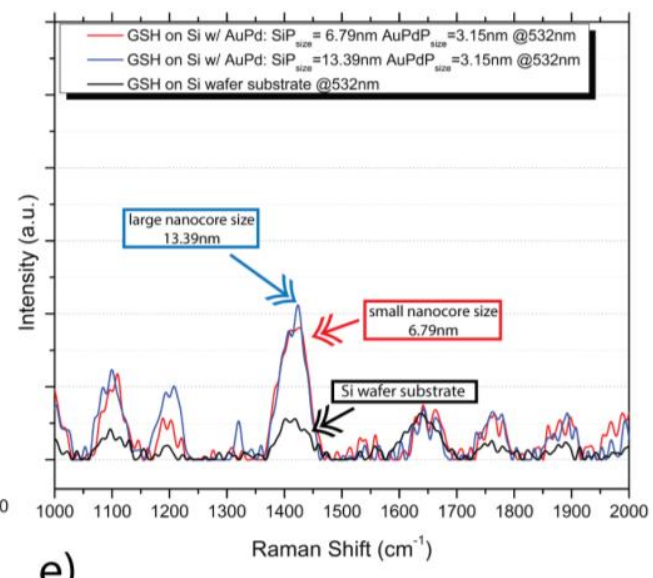

EF: All Sub Si @532nm, AuSi, AuPdSi w/GSH 10-6 M

e)

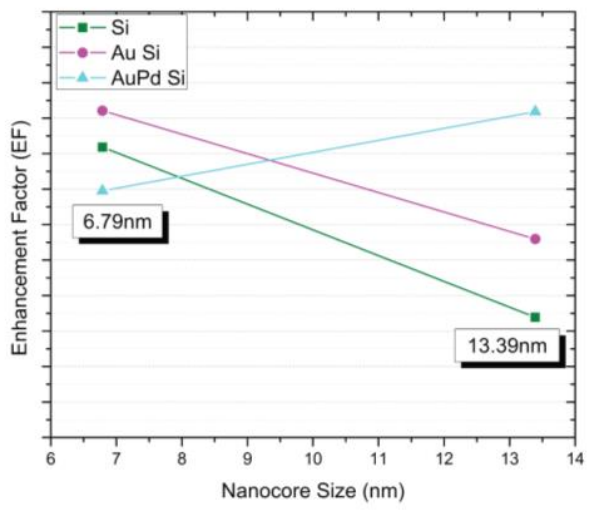


Figure 4-4: a) Raman Spectra of GSH biomolecule on Si wafer substrate, large Si nanocores, and small Si nanocores and b) EF values of GSH on small and large Si nanocores at micromolar $\left(10^{-6} \mathrm{M}\right)$ concentration c) Raman Spectra of GSH on Si nanocores w/ Au nanosatellites, and d)

Si nanocores w/ AuPd nanosatellites and e) the associated EF values
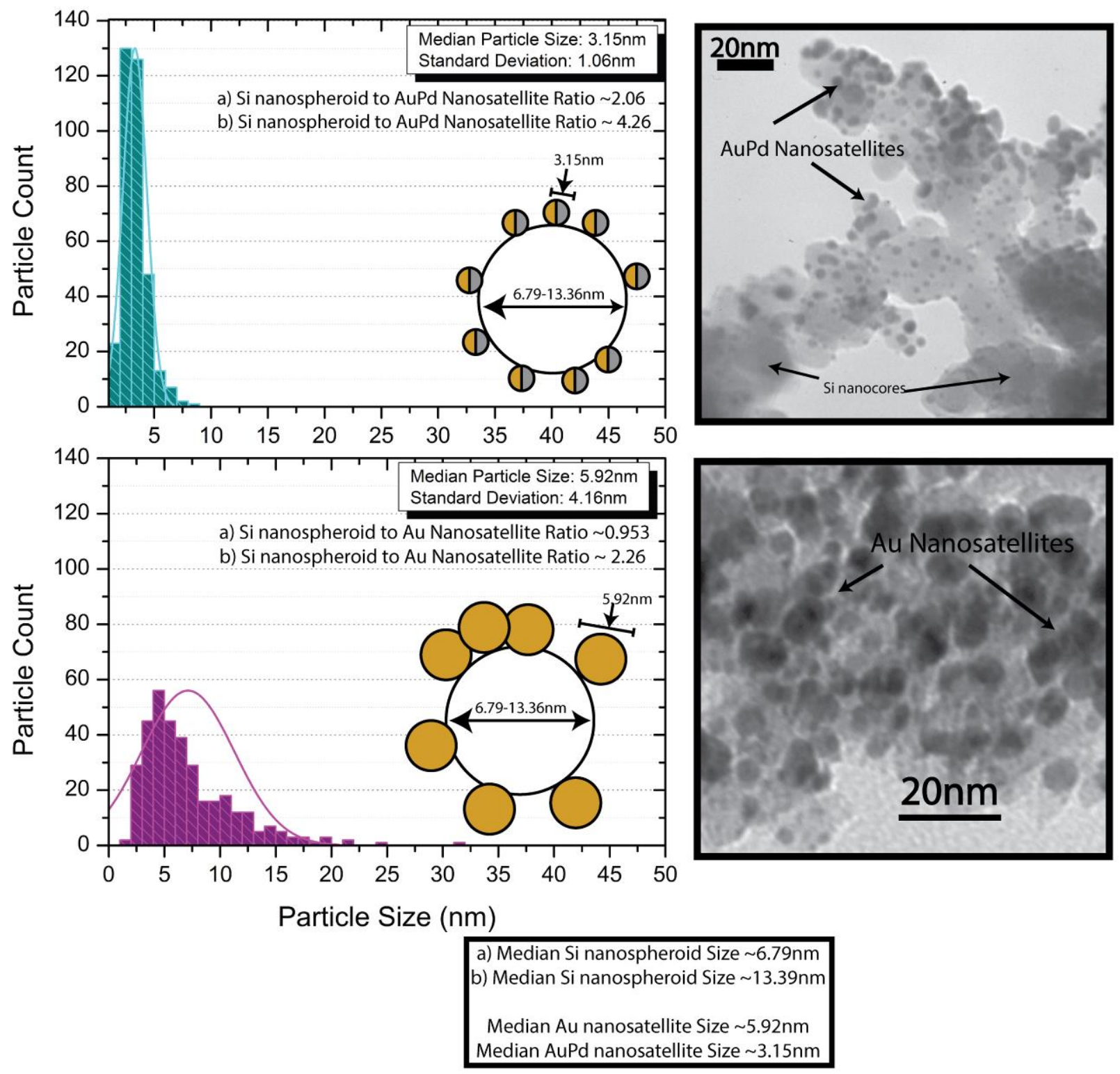

Figure 4-5: Size distribution plots for a) AuPd nanosatellites with b) TEM image of Si nanocore structure with AuPd nanosatellites and size distribution plot of c) Au nanosatellites with d) TEM image of Au nanosatellites on Si nanocore structure. 


\subsubsection{Nanocore superstructure Formation and SERS Enhancement}

The formation of the silicon nanocore superstructure used in this study, is unique to the ultrafast pulsed laser ion-plume (UPLIP) formation mechanism. The pulses from an ultrafast femtosecond pulsed laser, impinge on a $\mathrm{Si}$ wafer substrate surface, transfer a large amount of energy to the $\mathrm{Si}$ lattice which immediately ionizes the $\mathrm{Si}$ and forms an ion plume. Within this ion plume, $\mathrm{Si}_{+}$ions interact with each other to form polycrystalline/amorphous nanospheroids ${ }^{[4-25]}$ which condense and fall to the substrate; as more nanospheroids fall to the substrate surface, the nanospheroids will fuse and form a self-assembled three-dimensional nanocore network.

The nanosatellites are deposited onto the top of the 3D Si nanocore superstructure using a physical deposition method, whereby an Au or AuPd target is bombarded with $\mathrm{Ag}_{+}$ions within a UHV chamber leading to $\mathrm{Au}$ and $\mathrm{AuPd}$ atoms to be ejected from the target and depositing onto the $\mathrm{Si}$ nanocore network surface. Figure 4-6 shows an overview of these formation processes. 

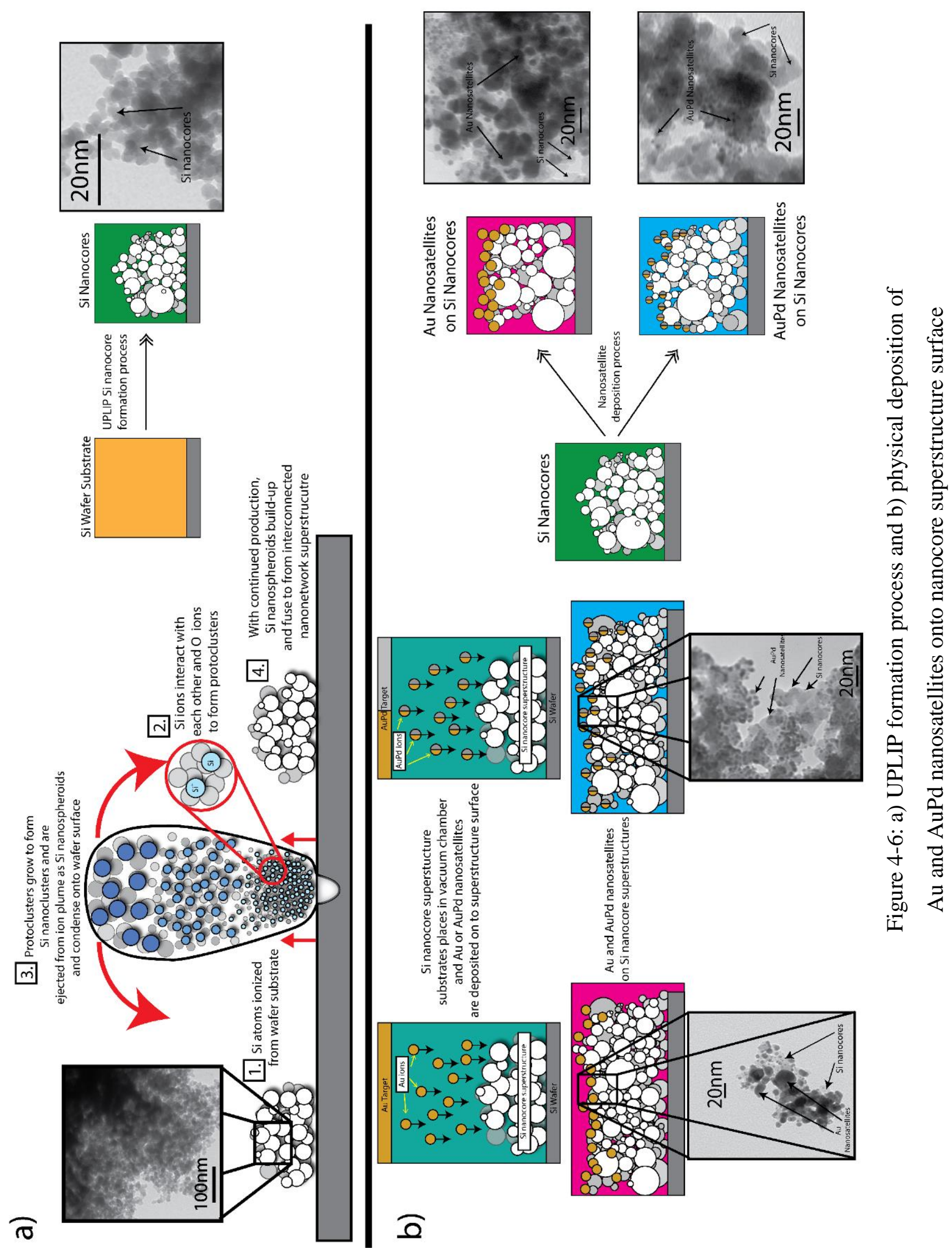

อ 
The particle size and particle size distribution curves in Figure 4-2 show that formation process has a significant effect on the size and distribution of the nanospheroids that comprise the nanocore structure. With one set of UPLIP conditions a median nanocore size of $6.79 \mathrm{~nm}$ and a standard deviation of $2.39 \mathrm{~nm}$ is generated, while with another set of conditions gives a $13.39 \mathrm{~nm}$ median nanocore size with a standard deviation of $5.57 \mathrm{~nm}$ is generated. The UPLIP process produces such variance in nanospheroid size and distribution due to the nature of the process itself. Upon ionization, a tremendous number of Si ions are ejected from the substrate surface to produce an ion plume, where collisions of ions lead to the formation of nanospheroids. The size of these is governed by the number of ion ejected from the substrate surface and the temperature of the ion plume which causes both nanospheroid growth and condensation.

While it has been shown that for noble metal nanoparticles a set particle size with narrow size distribution $^{[4-29]}$ is crucial for maximizing SERS efficiency, it has been observed that a narrow size distribution is not an essential requirement for providing a SERS signal with semiconductor Si nanostructures; this can be attributed to the differences between the mechanisms that govern the SERS enhancement from the semiconductor Si nanospheroids and the noble metal (Au, AuPd) nanosatellites. The nanosatellite size data also show that the Au and AuPd nanosatellites each have relatively narrow particle size distributions, with Au having a 5.92nm $\pm 4.16 \mathrm{~nm}$ median and AuPd having a $3.15 \mathrm{~nm} \pm 1.06 \mathrm{~nm}$ median nanosatellite size.

The XRD spectra show that the silicon nanospheroids that comprise the nanocore structure are indeed amorphous and polycrystalline in nature. This polymorphic nanospheroid structure is a further result of the UPLIP process; the rapid fluctuation in ion-plume temperature leads to regimes of amorphous nanocluster formation and polycrystalline nanocluster formation. With amorphous and polycrystalline nanoclusters forming within the ion-plume, these clusters will bind and form proto-polymorphic nanospheroids that condense and fall to the substrate surface. The rate at which amorphous nanoclusters and polycrystalline nanoclusters are formed is dependent on how the UPLIP process is programmed to interact with the Si wafer surface ${ }^{[4-25]}$. In this study, a specific set of UPLIP conditions is fixed to maintain a controllable comparison between nanocore enhancement spectra and to limit any variables that affect the SERS enhancement from the Si nanocore superstructures. 
Unlike most other SERS materials that use Si nanostructures as a base for NMN enhancement, the $\mathrm{Si}$ nanocore material that is formed is in and of itself a SERS enhancing nanomaterial. The Si nanocore material is SERS active due to the linked resonance enhancement mechanisms that are present in semiconductor SERS materials. ${ }^{[4-19]}$ The linked resonance mechanisms are a set of phenomena that cause SERS enhancement in semiconductor nanomaterials; these mechanisms are specific to semiconductor SERS nanomaterials due to the source of SERS enhancement from NMNs is primarily surface plasmon resonance (SPR). For instance, the works by Wang et.al ${ }^{[4-30]}$ and $\mathrm{Xu}$ et.al ${ }^{[4-31]}$ use $\mathrm{Au}$ nanoparticles on $\mathrm{MnFe}_{2} \mathrm{O}_{4}$ magnetic nanoparticles and $\mathrm{Au}$ nanoparticles on $\mathrm{ZnO}$ nanorods respectively are highly sensitive SERS nanostructures that are used for biosensing applications, but rely solely on the presence of NMNs for SERS enhancement. It is only recently that semiconductor nanostructures have become more widely researched as viable SERS sensing platforms, in addition to a fundamental theoretical basis ${ }^{[4-19]}$ for the underlying enhancement mechanisms of these semiconductor nanomaterials. Within this research area, there has yet to be an extensive investigation into multi-source biosensing SERS nanostructures that use both semiconductor nanostructures and NMNs as complementary and additive SERS enhancement sources. Convertino et.al ${ }^{[4-13]}$ have investigated the use of Au nanoparticles on Si nanowires for the detection of avidin biomolecules and Hong et.al ${ }^{[4-32]}$ have investigated the use of $\mathrm{Au}$ nanoparticles on $\mathrm{Fe}_{3} \mathrm{O}_{4} / \mathrm{ZnO}$ nanorices for the detection of goat-anti-human IgG antibody biomolecules, but this field still lacks an in-depth and comprehensive analysis of the Si-based SERS biosensing capabilities that NMN SERS has garnered.

The polymorphic Si nanocore superstructure that are created using the UPLIP process are highly SERS active substrates due to the unique material chemistry of the amorphous/crystalline nanospheroids and the highly branched and web-like nanonetwork structural morphology

These Raman spectra in Figure 4-3 show clearly that, the polymorphic Si nanocore structures have a significant enhancing effect that is contingent upon the morphology of the web structure and that at $532 \mathrm{~nm}$ the nanocore network is considerably SERS active. Additionally, in this study Au and AuPd nanosatellites are used to add a secondary boost the SERS enhancing properties of the polymorphic Si nanocore structure as seen in figure 3; these spectra show a comparison between the Raman spectra of the Si nanocore alone with the Si nanocores with Au nanosatellite and AuPd satellite modification. 
It is evident that the nanosatellite boosting mechanism has a profound effect on the Raman spectra. The intensity of the $520 \mathrm{~cm}^{-1} \mathrm{Si}$ peak is enhanced by the Si nanocore alone by a maximum EF value of 4.38 compared to the Si wafer substrate, while this same peak has a maximum EF value of 3.73 with Au nanosatellite boosting and an EF value of 12.14 with AuPd nanosatellite boosting. This increase is due to the multi-source SERS enhancement present with the nanosatellite boosted Si nanocore structures. The polymorphic Si nanocore exhibits SERS enhancement due to linked resonance mechanisms unique to semiconductor nanostructures. The linked resonance mechanisms are a set of SERS mechanisms that work in concert to produce greatly enhanced Raman spectra from the nanostructure surface. These mechanisms include charge-transfer (CT) resonance, exciton resonance and molecular resonance, each of which has distinct functionality. However, semiconductor nanomaterials are unable to produce a significant SPR enhancement contribution to Raman excitation because any induced SPR effect is in the infrared spectral range due to the photostability of semiconductor materials in the visible wavelength range. ${ }^{[-33]}$ Coincidentally, NMNs have a contrasting and supplementing SERS enhancement contributions. NMNs SERS mechanisms can be separated in to two distinct avenues, the chemical contribution and the electrochemical contribution. The chemical contribution is defines as the transfer of charge from an analyte molecule to the NMN and vice versa which enhances the Raman intensity of the analyte molecule. This contribution is largely ignored due to the much more substantial and overpowering electromagnetic contribution which is attributed to SPR. ${ }^{[4-34]}$ SPR is a mechanism by which, the photons from the Raman laser source cause a collective oscillation of the electrons on the surface of the NMN which creates a large electric field near the surface of the NMN which in turn allows for more Raman scattering and an increase in SERS intensity. It is these multi-source SERS contributions that are observed in the Raman spectra and EF presented in Figure 4-3. Figure 4-7 is a representation of the multi-source contributions from the primary Si nanocore and the secondary Au/AuPd nanosatellites. 


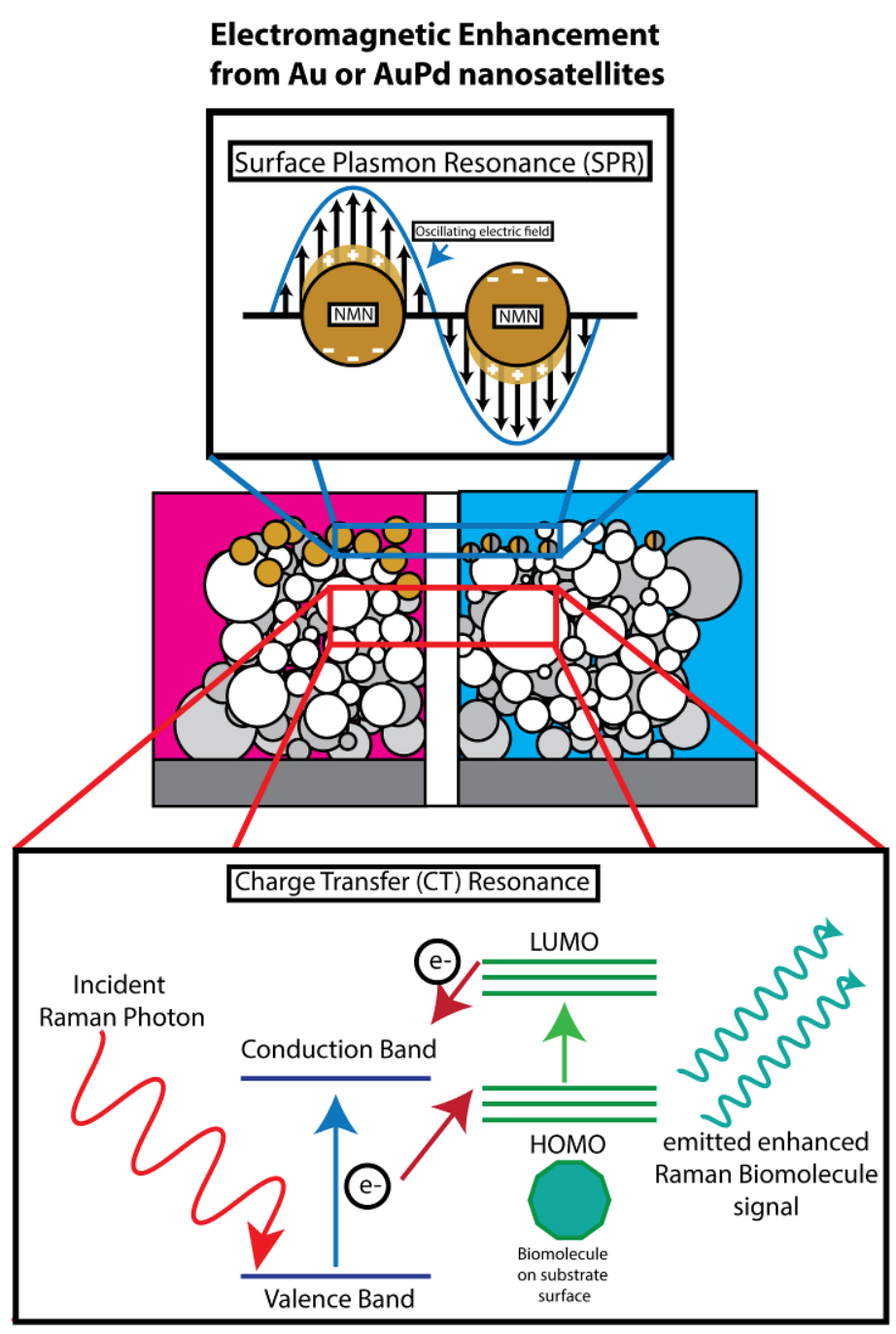

Linked Resonance Enhancement from Si nanocore superstructure

Figure 4-7: Two-factor contribution to SERS enhancement, the primary boost from the $\mathrm{Si}$ nanocores and the secondary boost from the noble metal nanosatellites

\subsubsection{SERS Biomolecular Enhancement}

\subsubsection{Polymorphic Si nanocore SERS Biosensing Efficiency}

As shown in Figure 4-3 polymorphic Si nanocores have a significant Raman activity compared to a Si wafer counterpart and establishes a basis for the enhancement of the nanoweb. With the application of a biomolecule onto the surface of nanocore structures, the goal is to validate this $\mathrm{Si}$ based nanostructure use as a practical biosensing platform. For this study, a tripeptide biomarker 
molecule, L-glutathione (GSH) which is present in living healthy and cancer cells and is an indicator of the amount of stress that the cell is undergoing. ${ }^{[4-35]}$ By sensing the levels of this biomarker molecule in cells, it is possible to determine if a cell is in the process of dying which is a sign of the efficacy of a cancer treatment. ${ }^{[4-27,4-28]}$ In this experiment GSH is applied at a specific concentration (micromolar $10^{-6} \mathrm{M}$ ) to the polymorphic Si nanostructures, and evaluated the Raman response associated with the biomolecule to determine if nanocore structures are indeed able to enhance the biomolecule spectra.

From the spectra in Figure 4-4 it is clear that the nanocore structure has a substantial effect on the biomolecule spectral intensity compared to the Si wafer substrate, in fact the GSH molecule is barely observable on the Si wafer substrate. At $1450 \mathrm{~cm}^{-1}$ at $532 \mathrm{~nm}$ there is a peak that is associated with the presence of GSH on the nanocore structure; this peak is not visible for neither the Si substrate spectra nor the Si nanocore spectra alone, therefore it can be expected that this peak only arises due to the presence of GSH. These peaks are not observable when GSH is applied to the Si wafer substrate, and only becomes visible on the nanocore structures. This peak for GSH from the works by Brambilla et.al is proposed to be due to $\delta(\mathrm{CH} 2)$ vibrational mode. ${ }^{[4-36]}$ The quantification of the enhancement of the GSH peak is achieved by calculation of the enhancement factor (EF). The EF equation used in this study is as follows:

$$
E F=\frac{I_{N S} / N_{N S}}{I_{s u b} / N_{\text {sub }}} \quad(\text { Equation } 4-1)
$$

Where $I_{N S}$ is the intensity of the GSH peak on the nanocore substrate, $I_{s u b}$ is the intensity of the GSH peak on the Si wafer substrate, $N_{N S}$ is the calculated number of GSH molecules within the Raman laser interaction volume on the nanocore structure and $N_{s u b}$ is the calculated number of GSH molecules in the Raman interaction volume on the Si wafer substrate. The calculation of $N_{N S}$ and $N_{s u b}$ are dependent on the material characteristics of the nanocore structure and the assumed Raman interaction volume. For the nanocore structure it is assumed that the density of the interconnected polymorphic Si nanospheroids is equivalent to a value experimentally determined by Cho et.al ${ }^{[4-37]}, 7.8 \mathrm{~m}^{2} / \mathrm{g}$, for porous Si nanofibres. The Raman interaction volume is assumed to be a hemisphere with a diameter of the laser spot size $19.154 \mu \mathrm{m}$. 
The EF values from Figure 4-4 show that a maximum EF for the $1450 \mathrm{~cm}^{-1} \mathrm{GSH}$ peak of $7.1 \times 10^{2}$ is achieved by the Si nanocores with $6.79 \mathrm{~nm}$ median nanocore size and an EF of $4.7 \times 10^{2}$ for the Si nanocores with $13.39 \mathrm{~nm}$ median nanocore size.

\subsubsection{SERS Biomolecular Enhancing using Au and AuPd Nanosatellite Boosting}

In this study, the addition of noble metal nanosatellites has been used to give a secondary boost the signal from the GSH biomolecule. Through physical vapor deposition, Au nanosatellites and AuPd nanosatellites have been deposited onto the top surface of the polymorphic Si nanocore structures. As has been observed, the addition of nanosatellites greatly improves the SERS signal of the Si nanocore structure (Figure 4-3) and it is with these secondary boosting nanostructures with which the means for a proposed multi-source biosensing enhancement mechanism is attained. The role of this multi-source enhancement mechanism has on the enhancement of the GSH molecules is dependent on the how sensitive the GSH molecule is to the SPR component of the enhancement mechanism, and how the morphology of the Au and AuPd nanosatellites affects a boost in the GSH enhancement. The effectiveness of the nanosatellite enhancement of the GSH spectra is determined through Raman spectral analysis of the GSH biomolecule on the individual nanostructure surface and quantified by the EF relative to a $\mathrm{Si}$ wafer substrate with $\mathrm{Au}$ or AuPd nanosatellites. To analyze the morphological dependence of the GSH enhancement, the nanocore size and size distribution of each nanocore created are measured along with and the size and size distribution of the nanosatellites in order to determine the role of a nanocore to nanosatellite size ratio on the EF values. The size distribution of the Si nanocore structures and the $\mathrm{Au}, \mathrm{AuPd}$ nanosatellites are measured using TEM images of these nanostructures. The diameter of a statistically significant number of each nanocore or nanosatellites are measured from seven different TEM images of each sample, from which the median and standard deviation of sizes was calculated as seen in Figure 4-5.

In terms of physical morphology, the Si nanocores structures are separated into three nanocore size/size distribution groupings, $6.79 \mathrm{~nm} \pm 2.39 \mathrm{~nm}, 13.39 \mathrm{~nm} \pm 5.57 \mathrm{~nm}$ and $16.86 \mathrm{~nm} \pm 7.95 \mathrm{~nm}$. The nanocore size distribution plots are shown in Figure 4-2.

Each of these groupings has either Au or AuPd nanosatellites deposited onto the nanocore surface. The size analysis results are shown in Figure 4-5, which are particle size distribution plots of both the Au nanosatellite diameter measurements and the AuPd nanosatellite diameter with sample 
TEM images showing the nanocore structure with nanosatellites on the surface. It is assumed that the deposition process is consistent regardless of nanocore substrate that the nanosatellites are deposited onto, therefore the nanosatellites sizes and distributions are assumed to be constant.

These plots show that the Au nanosatellites have a $5.92 \mathrm{~nm} \pm 4.16 \mathrm{~nm}$ size and the AuPd nanosatellites have a $3.15 \mathrm{~nm} \pm 1.06 \mathrm{~nm}$ size. Due to the method of deposition, the Au and AuPd nanosatellites will have different size/size distribution due the condensation and growth rate of the AuPd alloy. AuPd alloy source used in the nanosatellite physical vapor deposition process has a faster condensation rate and slower growth rate than the Au source, therefore the AuPd nanosatellites have a smaller median diameter compared to the Au nanosatellites. This physical morphological distinction plays a significant role in the enhancement of the GSH spectra

From theses spectra it is evident that the nanosatellites have a substantial effect on the GSH spectral intensity. The EF values in Figure 4-4 quantify the level of enhancement boosting the nanosatellites have on the GSH peak intensity compared to the spectra of the respective nanosatellites on the $\mathrm{Si}$ wafer substrate. The Au nanosatellites reach a maximum $\mathrm{EF}$ of $7.6 \times 10^{2}$ for the $1450 \mathrm{~cm}^{-1} \mathrm{GSH}$ peak and for the AuPd nanosatellite a maximum EF $7.6 \times 10^{2}$ is observed. It is clear that the effect of noble metal satellites is a significant increase in the GSH Raman signal as a result of the multi-source enhancement mechanisms. To quantify this boosting effect of the nanosatellite structure, the efficiency of the increase in enhancement of GSH with Au or AuPd nanosatellites relative to the bare $\mathrm{Si}$ nanocore structure is defined as the boosting efficiency. The boosting efficiency, is defined as the ratio of EF from the nanosatellite spectra to the EF of the $\mathrm{Si}$ only nanocore structure.

$$
\text { Boosting Efficiency }=\frac{E F \text { nanosatellite stuructre }}{E F \text { of Si nanocores }} \quad \text { (Equation 4-2) }
$$

The boosting efficiency of the nanosatellites is plotted in Figure 4-8. 


\section{a)}

Boosting Efficiency of AuPd Nanosatellites

Boosting Efficiency of Au Nanosatellites

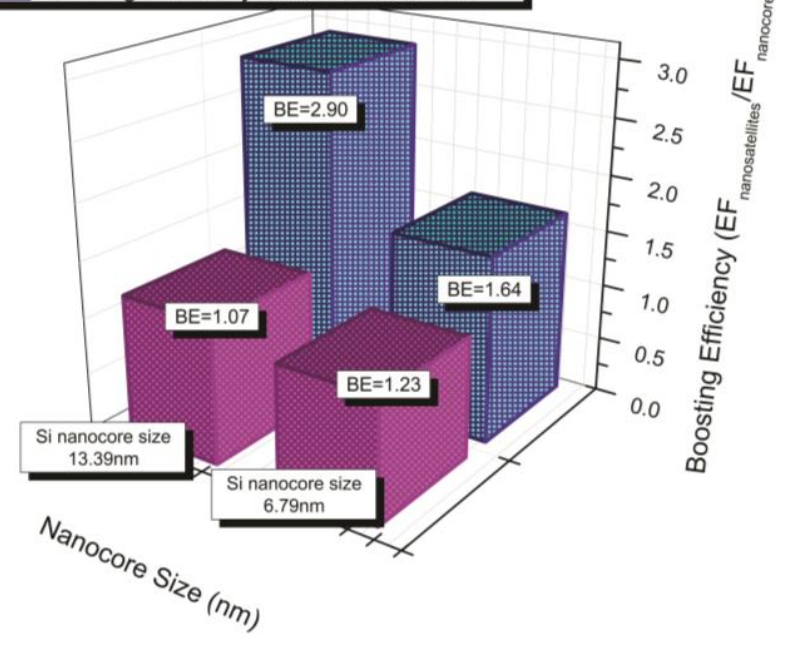

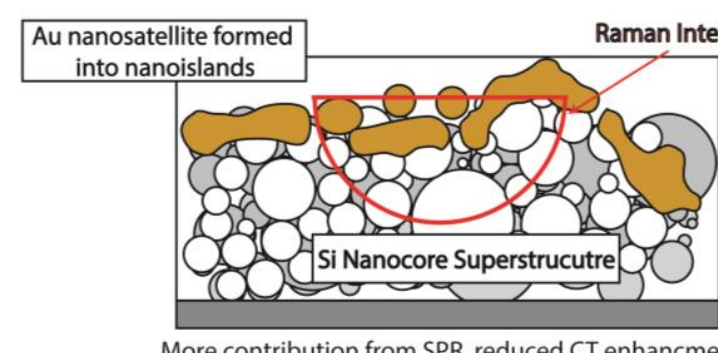

More contribution from SPR, reduced CT enhancment Boosting Efficiency: 23\%

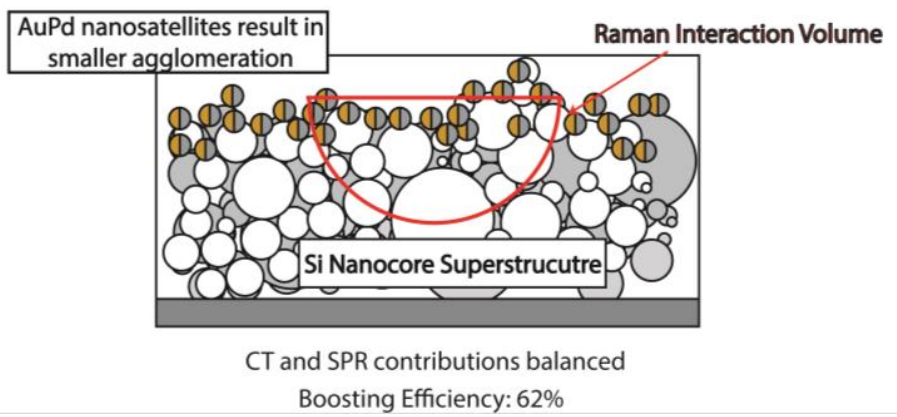

Figure 4-8: a) The boosting efficiency of the $1450 \mathrm{~cm}^{-1}$ GSH peak with Au and AuPd nanosatellites on $6.79 \mathrm{~nm}$ and $13.36 \mathrm{~nm}$ Si nanocores and b) schematic showing occupation of Raman interaction volume with Au and AuPd nanosatellites

The boosting efficiency shows that Au nanosatellites increase the EF for GSH by $23 \%$ and the AuPd increase the EF for GSH by $62 \%$ for the $13.39 \mathrm{~nm}$ Si nanocores. This result shows that the AuPd nanosatellites increase the GSH peak considerably more than the Au nanosatellites. The source of this increased boosting efficiency is posited to be the physical morphology of the nanosatellites on the polymorphic Si nanocore surface. It has been established that for noble metal nanoparticles, the size and size distribution of the nanoparticles plays a vital role in maximizing the SERS enhancement. In many other researchers work, the Au nanoparticle size that achieves the maximum EF ranges; for Qin et.al ${ }^{[4-38]}$ and Hong et.al ${ }^{[4-39]}$, the most enhancing Au nanoparticle size is $60 \mathrm{~nm}, 50 \mathrm{~nm}$ respectively. The Au nanosatellites that are deposited onto the Si nanocore are out of this range of size nanoparticle sizes which results in a boosting efficiency that is less than optimal. Since the vapor deposition method used to deposit the nanosatellites has a fixed deposition conditions, the nanoparticle size is somewhat invariable with a pure Au source target. However, by using an AuPd nanosatellite source target, the material properties are such that a faster condensation rate and slower growth and a prevention of island growth, leads to a much smaller nanosatellite size. Since the principle of SPR is heavily reliant on the size and volume of nanogaps 
for the surge in Raman scattered photons from the nanostructures surface the use of AuPd nanosatellites is advantageous. With a much smaller nanosatellite size, more AuPd nanosatellites cover the Si nanocore surface leading to a greater surface coverage ratio of nanosatellites compared to Au nanosatellites, which in turn increases the number of possible SERS hotspot zones and an increased Raman photon scattering potential. Another possible consequence of the smaller nanosatellite size is that, less of the underlying SERS active polymorphic Si nanocore structure is covered in nanosatellites. While both the nanosatellites and nanocore are contributing to the SERS signal, a balance between their contributions is require to optimize the GSH peak signal. It is proposed that since the Au nanosatellites have a larger size and tend to form larger agglomerates or nanoislands, more of the Raman interaction volume is occupied by the Au nanosatellites when compared to the AuPd nanosatellite coated nanocores. As a result, a greater percentage of the enhancement is attributed to the SPR mechanism than the CT enhancement mechanism, so much so that the CT mechanism is not optimally outputting SERS enhancement. It is suggested that with the AuPd nanosatellites, a greater balance is achieved between the two enhancement mechanisms which lead to the greater boosting efficiency seen in Figure 4-8 and enhancement factor seen in Figure 4-4.

\subsection{Summary}

In this study, it is shown that a nanocore network comprised of interconnected polymorphic $\mathrm{Si}$ nanospheroids is a viable SERS active biosensing nanomaterial for the detection of protein biomolecules. With the creation of a highly branched interconnected nanonetwork, the generated a SERS active semiconductor bioactive platform for biomolecule sensing is produced from a SERS inactive Si wafer substrate. This Si nanocore substrate is shown to be a substantially potent SERS biosensing nanomaterial by exhibiting primary boosting effect, resulting in a maximum enhancement factor of $7.1 \times 10^{2}$ for the GSH protein biomolecule though the linked resonance mechanisms associated with semiconductor SERS nanomaterials. Additionally, the GSH signal can become significantly boosted by the supplementation of noble metal nanosatellite structures secondary boosting structures to the surface of the polymorphic nanocore structure. These Au and the smaller AuPd nanosatellites provide an enhancement factor of $7.6 \times 10^{2}$ with the addition of the well-established SPR enhancement mechanism contributing to the GSH signal. It is the two-factor 
enhancement that leads to the boosting of the GSH signal that is observed in this study and results in a boosting efficiency of $23 \%$ and $62 \%$ for $\mathrm{Au}$ and AuPd nanosatellites respectively. 


\section{Chapter 5}

\section{Towards Universal $\mathrm{Si}$ SERS-based nanobioprobe materials through engineering quantum-scale defects within self-assembled Si-only disordered 3D nanomesh structures}

In this study, a potential universal SERS biosensing nanoprobe material is generated by engineering quantum-scale defects in a self-assembled Si nanomesh structure. Through ionization of a single crystal defect-free $\mathrm{Si}$ wafer highly amorphous/nanocrystalline $\mathrm{Si}$ nano-orbs are constructed and coalesce to create a self-assembled connective 3D nanomesh structure with observed enhanced SERS nanobiosensing activity. By amending the ionization of a single crystal defect-free Si wafer through the externally influencing ion interactions within the formed laserion plume, nanomesh structures with quantum-scale nanograin disorder and surface nanovoids are generated. The resultant disordered Si nanomesh structures, exhibit enhanced Raman activity that is dependent on these morphological and crystallographic quantum scale defect characteristics. The SERS nanobiosensing sensitivity with the observed quantum-scale disordered Si nanomesh structures was evaluated using of a tripeptide biomarker L-glutathione (GSH). It is observed that an EF value of $\sim 10^{2}$ for the GSH biomolecules can be achieved by influencing ion interaction mechanism. Additionally, the SERS detection of multiple biomolecules with the quantum scale disordered nanomesh structures indicates the potential use of these unique Si only nanomaterials as a universal biosensing nanoprobe devices. 


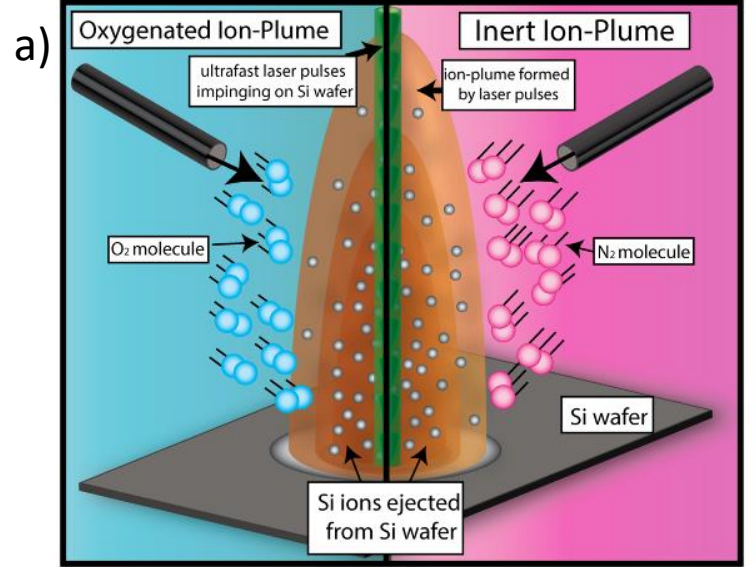

I-glutathione (GSH)

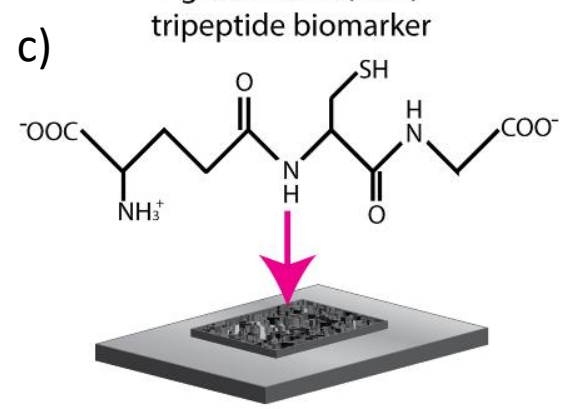

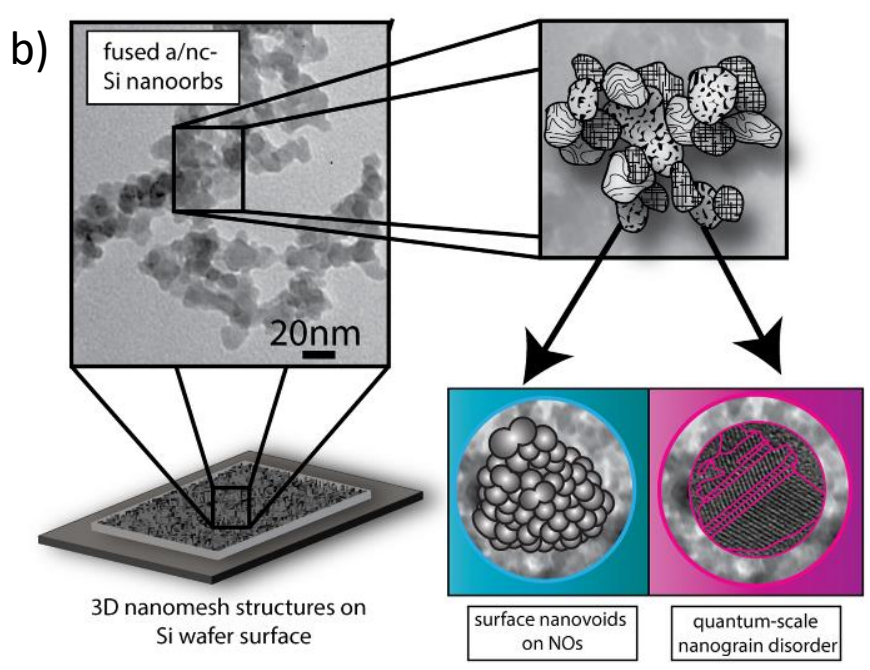

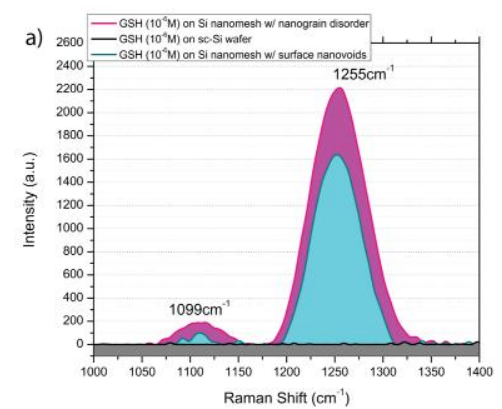

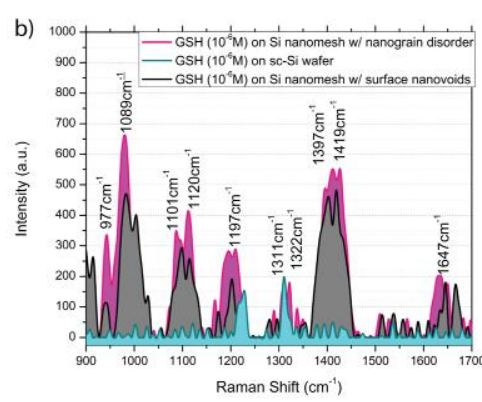

Figure 5-1: Overall Schematic showing a) ion interaction amendmentation to form inert or oxygenated ion-plumes, b) types of engineered quantum scale defects within NO structure, c) application of biomolecule and subsequent SERS enhancement spectra

\subsection{Introduction}

For biosensing applications employing surface enhanced Raman scattering (SERS), the use of nanostructured noble metal materials have been extensively researched due to the well-established surface plasmon resonance (SPR) enhancement effect unique to these nanomaterials ${ }^{[5-1]}$. These noble metal nanostructures have been fabricated using various techniques including chemical synthesis ${ }^{[5-2]}$, nanolithography ${ }^{[5-3]}$, and physical deposition ${ }^{[5-4]}$ which have been modified and optimized to excite the detection of various bioanalytes; noble metal nanorods ${ }^{[5-5,5-6]}$, nanoparticles ${ }^{[5-7]}$, and nanowires ${ }^{[5-8]}$ have all shown SERS detection of biomolecules due to the SPR effect. These noble metal nanostructures (NMNs) have been able to provide a significant enhancement in the detection of various biomolecules including proteins ${ }^{[5-9]}$, cancer $^{[5-10]}$ and $\operatorname{mRNA}^{[5-11]}$. 
Recent advances have shown that, semiconductor and transition metal oxide nanostructures hold considerable potential as SERS enhancing sources, including $\mathrm{WO}_{3-\mathrm{x}}{ }^{[5-12]}, \mathrm{TiO}_{2}{ }^{[5-13]}, \mathrm{CuO}^{[5-14]}$, $\mathrm{ZnO}^{[5-15]}$ and $\mathrm{Si}^{[5-16]}$. These alternative SERS enhancing nanomaterials could potentially reduce or replace noble metals in biosensing devices and by overcoming limitations associated with NMNs including lack of reproducibility of SERS enhancement and specific biocompatibility issues. While some researched SERS biosensing nanomaterials have both noble metal and semiconductor/metal oxide (S/MO) nanostructures ${ }^{[5-17-5-19]}$, the noble metals serve as the primary SERS source while the S/MO acts merely as a framework or support for the SERS active NMNs. While NMNs provide SERS enhancement through SPR, S/MO provide SERS enhancement through linked resonance mechanisms ${ }^{[5-20]}$, which include exciton resonance and charge transfer resonance ${ }^{[5-21,5-22]}$. These resonance effects are referred to as the chemical contribution for NMN enhancement and are considered irrelevant for these nanomaterials but have been shown to be the primary SERS enhancement source for S/MO nanostructures ${ }^{[5-22,5-23]}$. The most studied of these linked resonance mechanisms is charge transfer resonance, which is the process of transferring electrons from a semiconductor to an analyte molecule and vice versa and is dependent on their bandgap and highest occupied molecular orbital-lowest unoccupied molecular orbital (HOMOLUMO) energy difference respectively ${ }^{[5-24]}$.

Si nanostructures are typically thought of inactive contributors to SERS enhancement because most fabrication techniques are unable create the material chemistry required to activate the SERS properties of Si. In a majority of studies that use "Si-based" SERS nanostructures, Si nanowires ${ }^{[5-}$ ${ }^{25]}$, Si nanocones ${ }^{[5-18]}$, Si nanowells ${ }^{[5-26]}$, or porous Si nanostructures ${ }^{[5-27,5-28]}$ are coated with $\mathrm{Au}$ or Ag nanoparticles ${ }^{[5-25,5-29,5-30]}$ for SERS activation rather than creating a SERS active material from a strictly Si nanostructure. In our previous studies, Si nanostructures have been shown to be highly SERS active for both chemical and biosensing applications, but only when generated using an ultrafast femtosecond pulsed laser ion reaction mechanism ${ }^{[5-16,5-31]}$. During this formation, a defect -free single crystal Si wafer and the surrounding air is ionized with the femtosecond laser and forms an ion plume containing these ions. The ions in the plume interact and bond to form nanoclusters which grow, condense as nano-orbs and finally fall onto the wafer surface. These fallen nano-orbs will fuse together to form a highly porous 3D nanomesh-like structure whose crystal structure and morphology are dependent on how the laser pulses interact to ionize the substrate and how external factors impact the ion interactions. The observed material chemistry 
of this $\mathrm{Si}$ nanostructure is not possible, to our knowledge with other fabrication techniques, because this initial ionization process completely reforms the material chemistry of the $\mathrm{Si}$, creating both nanocrystalline and amorphous Si regions within an individual nano-orb (NO), rather than a single crystal nanostructure.

Recent studies have shown that the SERS activity of S/MO nanostructures can be significantly enhanced by engineering defects within the S/MO nanostructure. Cong et al. ${ }^{[5-23]}$ and $\mathrm{Wu}$ et al.

${ }^{32]}$ have shown that by introducing point defects into S/MO nanostructures in the form of oxygen vacancies, very high enhancement factor values $\left(10^{5}-10^{7}\right)$ and ultrahigh sensitivity $\left(10^{-7} \mathrm{M}-10^{-8} \mathrm{M}\right)$ can be observed for chemical analytes.; these reported EF values and sensitivities are on par with NMNs. In addition Ji et.al ${ }^{[5-33]}$ have shown that $\mathrm{O}_{2}$ vacancies on $\mathrm{TiO}_{2}$ nanoparticles acts as coordination sites for molecules which causes greater CT between the nanoparticles and analyte molecules. A similar effect can be proposed in this study, the quantum scale defects within the Si NO can act as SERS enhancing sources for the detection of biomolecules. Coupling these observations with the observed SERS activity of hybrid a/nc Si nanomesh networks, in this study a method of introducing quantum scale defects into a Si nanostructure as a means of evaluating the role of sub-nano defects on the SERS nanobiosensing characteristics of Si nanomesh structures is proposed. These effects of near-quantum scale disorder within a Si nanostructure have yet to be studied as a SERS enhancing source for nanobiosensing applications

In this study, near-quantum scale defects are introduced into a/nc-Si NOs to determine the SERS nanobiosensing characteristics, in order to further the understanding of how this uniquely disordered Si material chemistry affects the detection of biomolecule analytes. By amending the ion interactions within the laser-ion plume near quantum-scale disorder is induced within resultant a/nc-Si NOs that comprise the Si nanomesh. It was found that though amendmentation of the ion formation, two types or near-quantum scale defects are introduced into the Si nanomesh structure. The first being an internal disorder of the nanograins with the individual NOs that comprise the nanomesh. The second being an external surface disorder of the NOs in the form of nanovoids. An investigation of how these near-quantum disorders within these a/nc Si nanomesh can affect the SERS selectivity with a biomolecule analyte, L-glutathione (GSH) has been conducted along with an evaluation of the wavelength dependence and sensitivity. GSH is a tripeptide biomarker which acts as a signalling molecule for diseases neurodegenerative disorders, cancer and cystic 
fibrosis among others ${ }^{[5-34]}$. While GSH has been detected using SERS techniques, very limited enhancement and specificity for this biomolecule been reported, even with plasmonic noble metal nanostructures. Additionally, the detection of biomolecules tryptophan (Trp), cysteine (Cys) and methionine (Met) with the near-quantum disordered nanomesh structures was evaluated to determine the potential of these Si nanomesh structures as a universal biosensing nanoprobe. These biomolecules have been identified as disease signalling biomolecules for various diseases ${ }^{[5-35,5-36 \text {, }}$ ${ }^{5-37]}$ and there have yet to be studies conducted using Si-only nanostructures as a SERS nanoprobe for detecting any of these biomolecules.

\subsection{Experimental Method}

The generation of the nanomesh structures was achieved using a Clark-MXR IMPULSE pulsed Yb-doped fibre amplified femtosecond laser to ionize a $0.02-\Omega \mathrm{cm}$ p-type silicon [100] wafer substrate. This ultrafast pulsed laser formation mechanism is kept consistent by maintaining laser wavelength (1030nm), polarization (circular), average laser power (16W) and laser pulse width (214fs). To consistently create an even distribution of nanomesh on the wafer surface, a piezodriven raster system was used to ionize the $\mathrm{Si}$ wafer in a $300 \times 300$ point array with $0.25 \mathrm{~mm}$ spacing designed by EzCAD software. To control and test the range of nanostructures that could be viable SERS active nanomesh structures, the laser pulse repetition rate $(4 \mathrm{MHz}$ and $26 \mathrm{MHz})$ is varied to alter the ionization energy of the laser-ion plume. In order to modify the $\mathrm{Si}-\mathrm{O}$ ion interactions for this nanomesh formation mechanism gaseous species of $\mathrm{N}_{2}$ and $\mathrm{O}_{2}$ are introduced into the ionplume formed by the pulsed laser. The gases are injected into the laser-wafer interaction zone through seven individual nozzles which evenly surround the ionization zone. The gaseous species are introduced at a flow rate of $0.2 \mathrm{MPa}$.

The physical morphology of the resulting nanomesh structures were imaged using a Hitatchi H7000 transmission electron microscope (TEM) on copper mesh grids. The size and size distribution of the individual NOs observed in these images were manually measured and tabulated using ImageJ software. The XRD crystallographic analysis of the nanomesh structures was performed using a Rigaku Miniflex 600 diffractometer. A $2 \theta$ scanning range of $15^{\circ}$ to $60^{\circ}$ was used to acquire the relevant peaks associated with the Si nanostructures. 
To acquire the Raman spectra of both the nanomesh structures and of the protein biomolecule on the nanomesh structures a Bruker Optics SENTERRA Raman confocal microscope with a 10x magnifying lens. Both 532nm and 785nm Raman spectra of the nanomesh and biomolecule were obtained in this study using $5 \mathrm{~mW}$ and $25 \mathrm{~mW}$ power respectively. To achieve a consistent significant signal a 10s integration time was used and to maintain consistent signal response, 3 iterations were acquire for each Raman spectra in this study. For the acquisition of L-glutathione (GSH) Raman spectra, $10 \mu \mathrm{L}$ of GSH of $10^{-6} \mathrm{M}$ or $10^{-9} \mathrm{M}$ concentration was applied to the relevant nanomesh structure just prior to Raman acquisition.

\subsection{Results and Discussion}

\subsubsection{Nanomesh formation by multi-photon ionization of solid Si substrate}

The nanomesh structures created in this study are generated using an ionization process that a transforms a defect-free single crystalline $\mathrm{Si}$ (sc-Si) wafer into a three-dimensional self-assembled nanomesh network of fused amorphous/nanocrystalline $\mathrm{Si}(\mathrm{a} / \mathrm{nc}-\mathrm{Si}$ ) NOs on top of this same sc-Si wafer. To transform a sc-Si wafer into this complex polymorphic nanostructure, the sc-Si wafer must be broken down into its core ions and reformed within the ion-plume. The only method to our knowledge that can create this type of nanostructure with this specific material chemistry is through multi-photon ionization using an ultrafast femtosecond pulsed laser (UFPL) formation mechanism. The UPFL process is highly programmable and can be manipulated to produce nanostructures of a desired morphology and composition by altering the properties of the impinging laser pulses. The femtosecond laser pulses that strike the substrate surface will transfer such high energy to the sc-Si wafer substrate, that there is complete ionic breakdown of the solid crystalline structure of the sc-Si wafer to form a cloud of $\mathrm{Si}$ ions just above the wafer surface. Not only does the solid sc-Si wafer ionize, the surrounding gases, principally $\mathrm{O}_{2}$ molecules are ionized as well.

This results in an ion plume composed of $\mathrm{Si}$ and $\mathrm{O}$ ions which combine and react with each other. The outcome of this is the formation of amorphous $\mathrm{Si} / \mathrm{SiO}_{2}$ nanoclusters and nc-Si nanoclusters which assemble to form the incross NOs which condense and collapse onto the Si wafer surface. Figure 5-2 shows how the a/nc-Si nanomesh structures are formed in this experimental study. In Figure 5-2a, the ultrafast pulsed laser immediately ionizes the Si wafer surface leading to Si ions 
being rapidly ejected from the wafer surface. These Si ions collide with each other to form protonanoclusters of amorphous $\mathrm{Si}$ and $\mathrm{SiO}_{2}$ or nanocrystalline $\mathrm{Si}^{[5-16]}$. These proto-nanoclusters agglomerate and form amorphous/crystalline Si NOs which condense and fall out of the ion-plume onto the sc-Si wafer substrate. These NOs fuse together on the Si wafer surface and form a selfassembled three-dimensional nanomesh-like structure. Figure 5-2b shows SEM, TEM and HRTEM images of the 3D nanomesh structure, the fused a/nc-Si NOs that comprise the nanomesh and the hybrid nature of the NOs respectively.

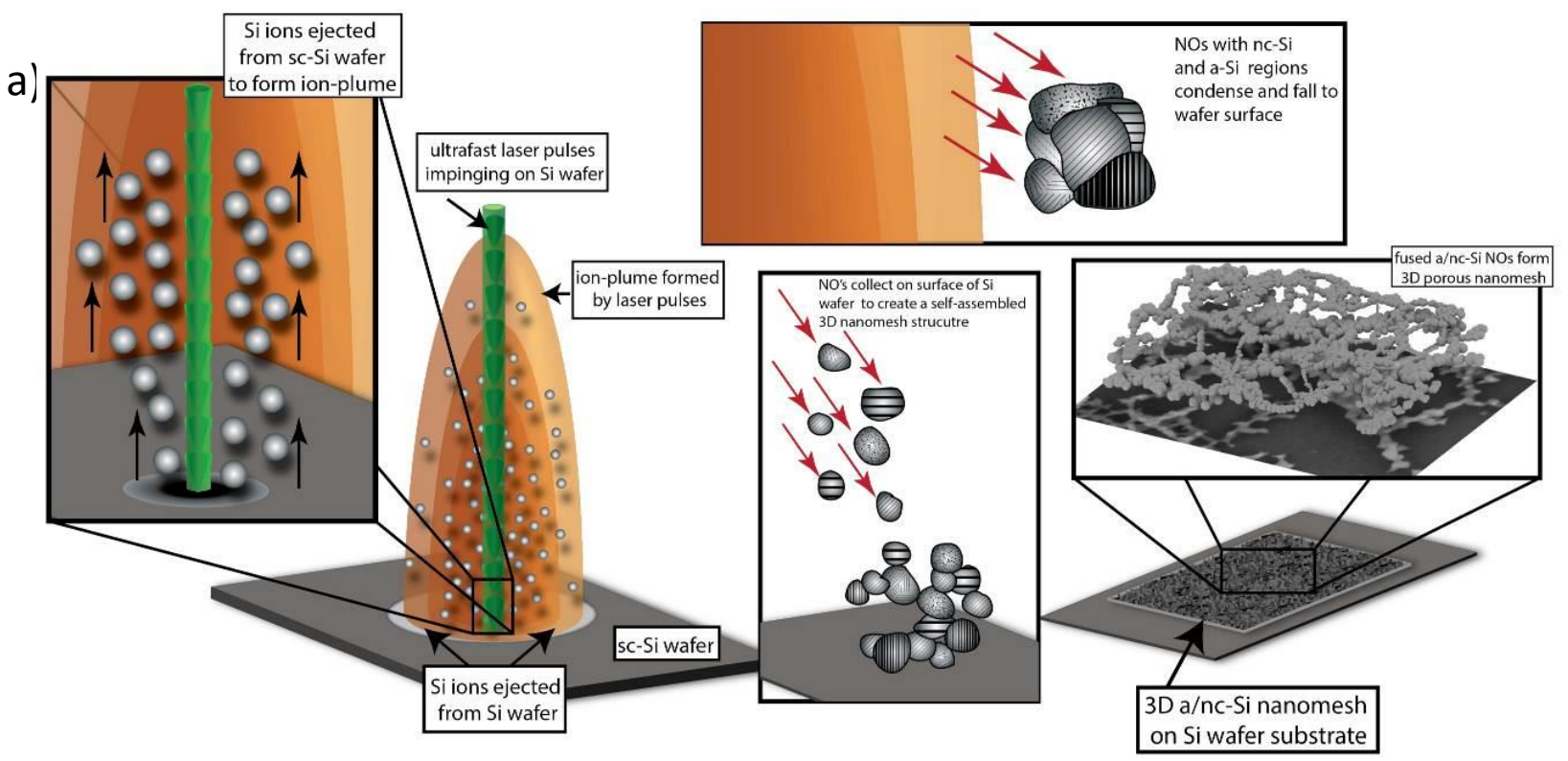

b)

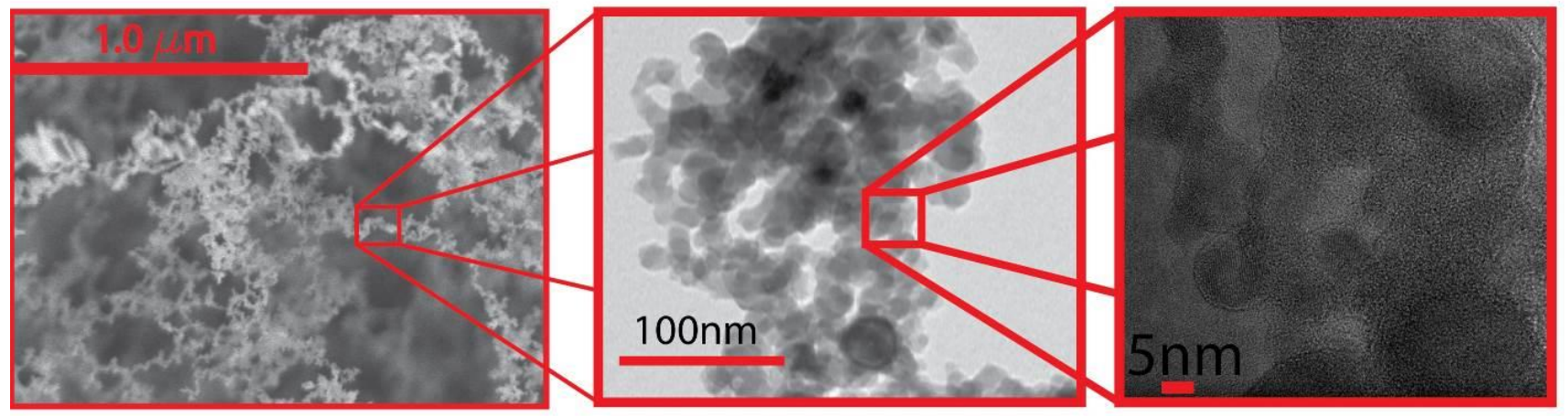

Figure 5-2: a) femtosecond laser ionization of a defect-free silicon wafer, NO ejection from ion plume, NOs fuse together on Si wafer surface to form 3D nanomesh b) images of nanomesh morphology and hybrid Si NOs 
Since the ion-plume conditions are variable depending on the laser-substrate interaction conditions, the nanomeshes in this study are classified in terms of a quantity known as the ionization coefficient $^{[38]}$. This value is calculated as:

$$
\alpha=\left(I / t_{\text {crit }}\right) \ln \left(N_{C} / N_{0}\right) \quad \text { (Equation 5-1) }
$$

Where $I$ is the laser energy density, $t_{c r i t}$ is the duration of the femtosecond pulse required to achieve ionization, $N_{C}$ is the electron density of the ion-plume and $N_{O}$ is the number of electrons in the wafer before ionization. From this equation the ion-plume energy of the different laser conditions used in this study can be quantified. High ionization energy is defined as $a=12.9 \times 10^{13}$ and low ionization energy defined as $2.50 \times 10^{13}$.

In this study the influence of inducing disorder during the ionization of $\mathrm{Si}$ is being investigated to determine how the resulting 3D polymorphic disordered Si nanomesh structures allow for SERS detection of a biomolecule species. Of particular interest is the determination of how these quantum scale disorders can be engineered to functionalize the SERS activity of a Si nanostructure for high sensitivity nanobiosensing applications. To achieve this, gaseous nitrogen or oxygen species are introduced into the ion plume externally from a pressurized source. $\mathrm{N}_{2}$ was chosen due to the very high ionization energy associated with this molecule $(15.58 \mathrm{eV})$ therefore $\mathrm{N}_{2}$ acts as an unreactive species in the ionization and nanostructure formation process. $\mathrm{O}_{2}$ and $\mathrm{Si}$ have ionization energies $12.07 \mathrm{eV}$ and $8.152 \mathrm{eV}$ respectively and as such are the only reactive species in the laserionization process. To supplement the oxygen interaction diatomic $\mathrm{O}_{2}$ gas is introduced into the ion-plume to further increase the concentration of oxygen ions in the plume due to the lower ionization energy of $\mathrm{O}_{2}$ molecules. The ion interaction influence reaction being investigated in this study is represented by:

$$
\begin{gathered}
s c S i^{(\text {solid })}+\mathrm{O}_{2}^{(\text {gas })}+N_{2}^{(\text {gas })}+\operatorname{air}(\text { gas }) \stackrel{\text { UFPL ionization }}{\longrightarrow}\left(\mathrm{Si}^{2+}+\mathrm{Si}^{4+}\right)^{i o n s}+\left(\mathrm{O}^{2-}\right)^{\text {ions }}+\mathrm{N}_{2}^{(\text {gas })}+e^{-} \\
s c S i^{(\text {solid })}+\mathrm{O}_{2}^{(\text {gas })}+\operatorname{air}^{(\text {gas })} \stackrel{\text { UFPL ionization }}{\longrightarrow}\left(\mathrm{Si}^{2+}+\mathrm{Si}^{4+}\right)^{i o n s}+\left(\mathrm{O}^{2-}\right)^{i o n s}+e^{-}
\end{gathered}
$$

\subsubsection{Characterization of disordered Si nanomeshes}

An investigation of the material and morphological characteristics of the disordered a/nc-Si nanomesh was carried out using a series of characterization techniques including, TEM/HRTEM imaging, XRD analysis and Raman spectroscopy; these techniques reveal substantial amount of 
information regarding how the ion interactions can be altered to induce structural and morphological disorder and distinct properties of the resultant disordered Si nanomesh structures.

Figures 5-3 and 5-4 show the characterization data obtained for the nanomeshes formed under inert ion-plume conditions and oxygenated ion-plume conditions respectively.

a)

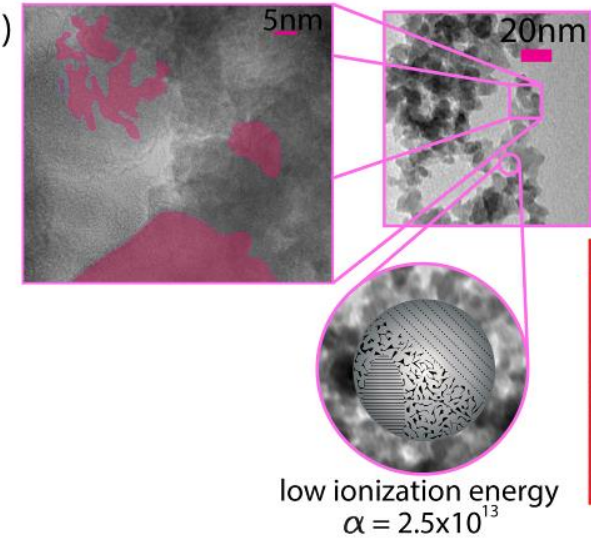

b)
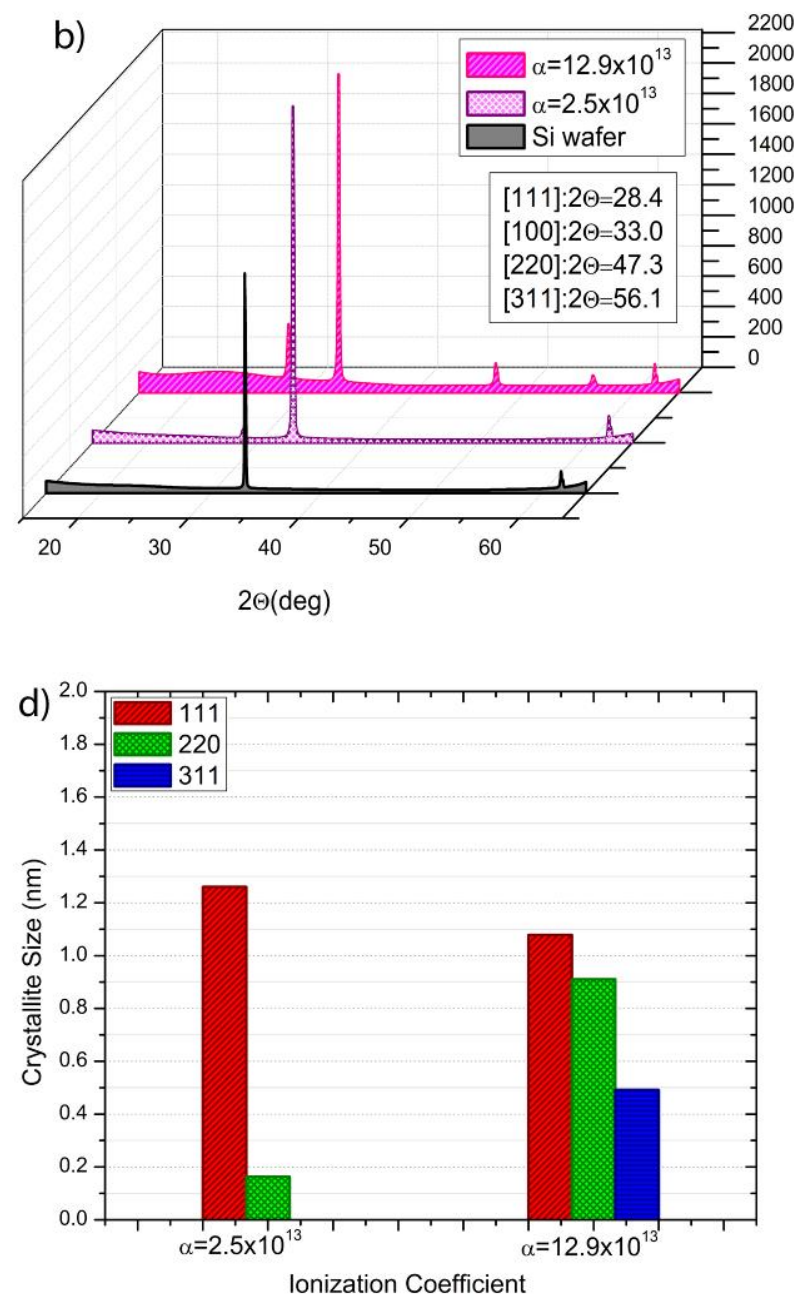

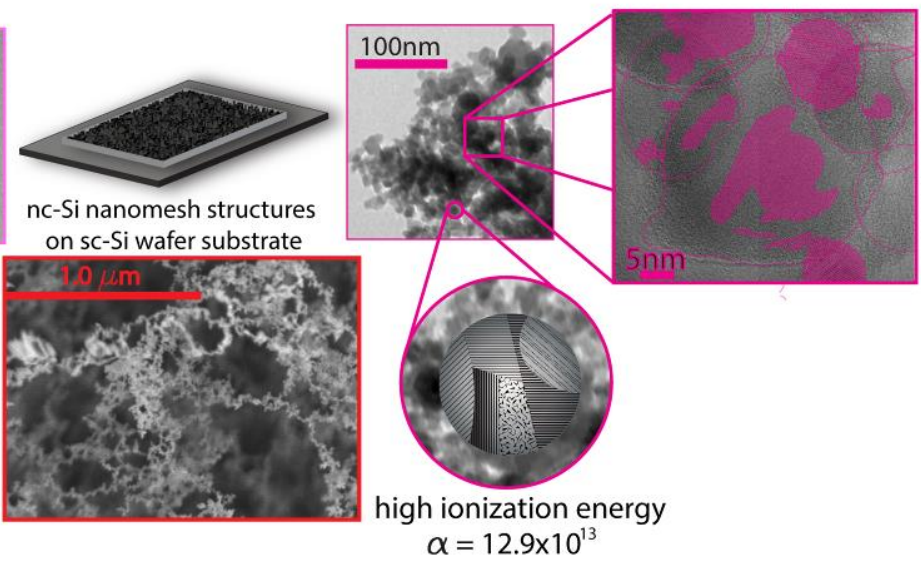

c)
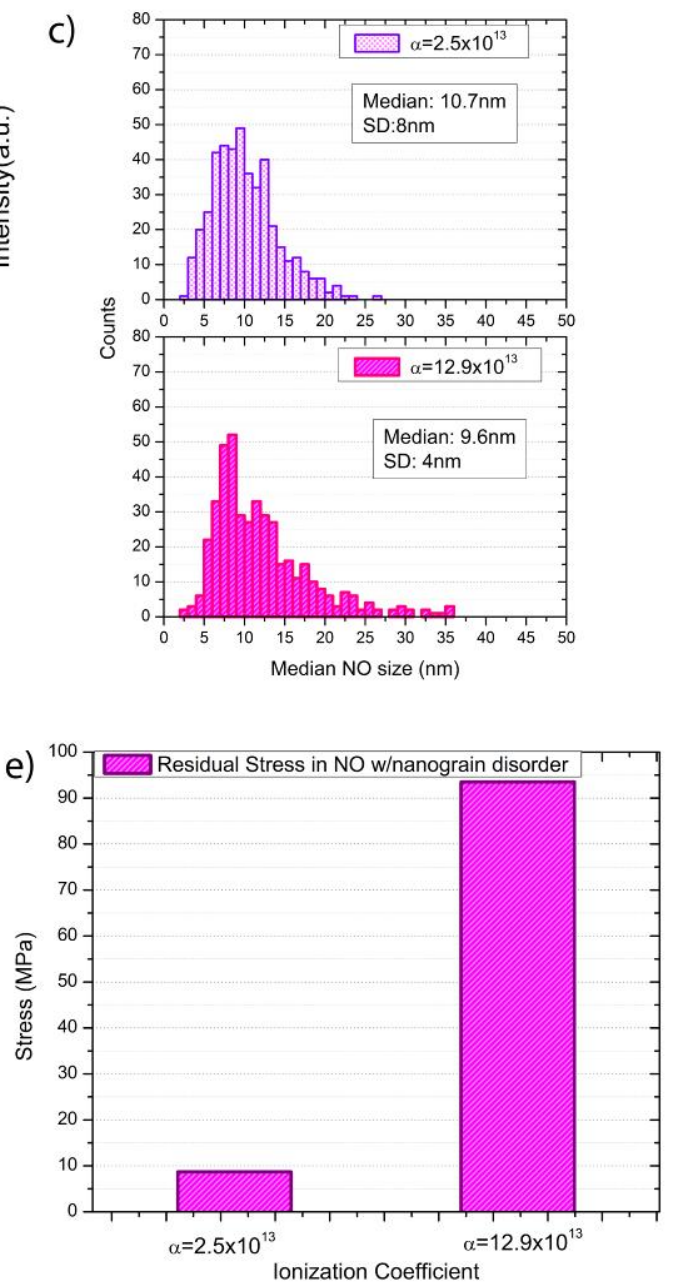
Figure 5-3: a) HRTEM analysis of quantum scale defects within NO formed under inert-ion plume conditions and associated b) XRD spectra, c) NO size distribution d) calculated crystallite size and e) calculated residual stress

a)

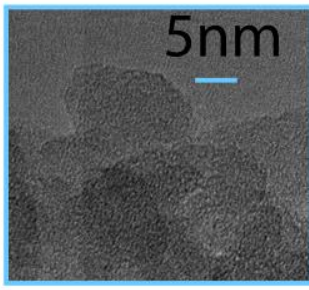

lowionization energy $\alpha=2.5 \times 10^{13}$

b)

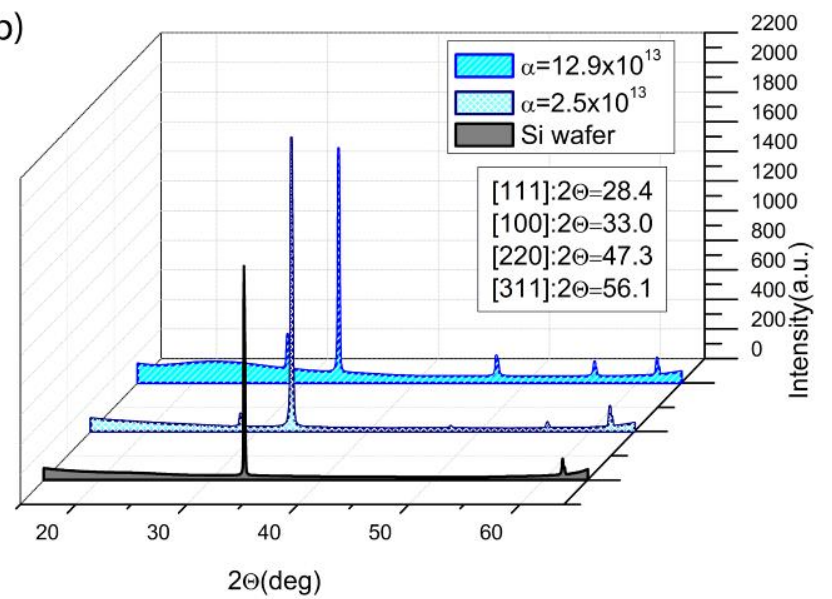

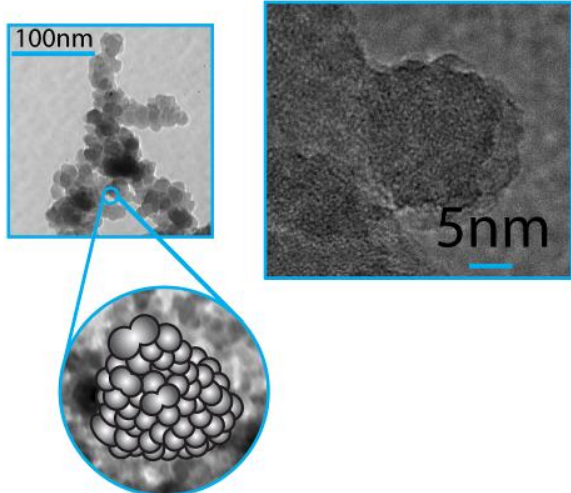

high ionization energy $\alpha=12.9 \times 10^{13}$

c)

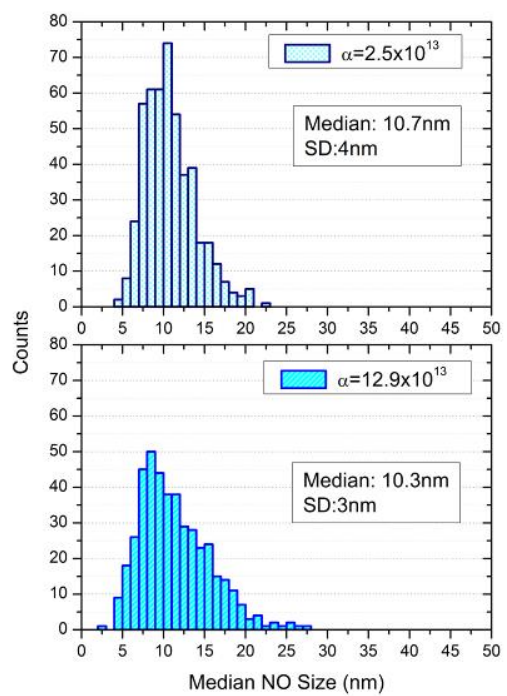

d)

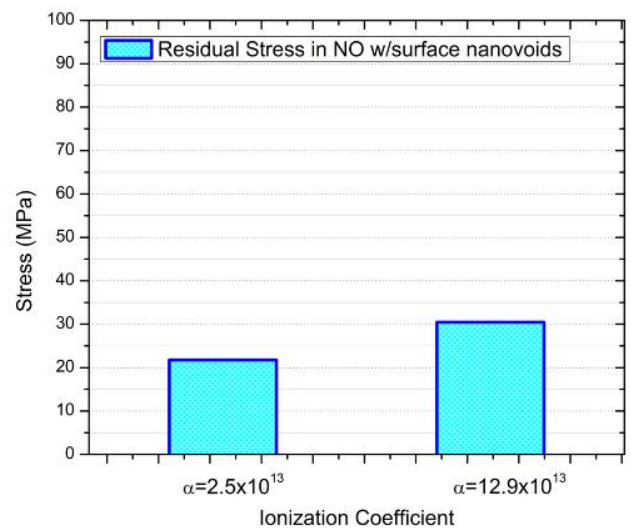


Figure 5-4: a) HRTEM analysis of quantum scale defects within NO formed under oxygenated ion plume conditions and associated b) XRD spectra, c) NO size distribution d) calculated residual stress

\subsubsection{Analysis of Quantum-scale defects}

To determine how the ion interactions affect the formation of disorder within the Si nanomesh structures, HRTEM imaging of the nanomeshes was performed to analyze the structure of the individual NOs that comprise the nanomesh structures. The images (Figure 5-5) reveal that two distinct categories of disorder are induced within the nanomeshes; internal near-quantum scale nanograin disorder and external surface nanovoid disorder. Figure 5-5c shows multiple defects within the crystalline structure of an individual NO and Figure 5-5d shows the presence of a highly disordered surface structure and the formation of nanovoids on the NO surface. These two distinct forms of disorder, manifest as a result of the amendmentation of the ion-plume conditions; the internal nanograin disorder is formed within an inert ion plume and the external surface nanovoid disorder is formed within an oxygenated ion plume.

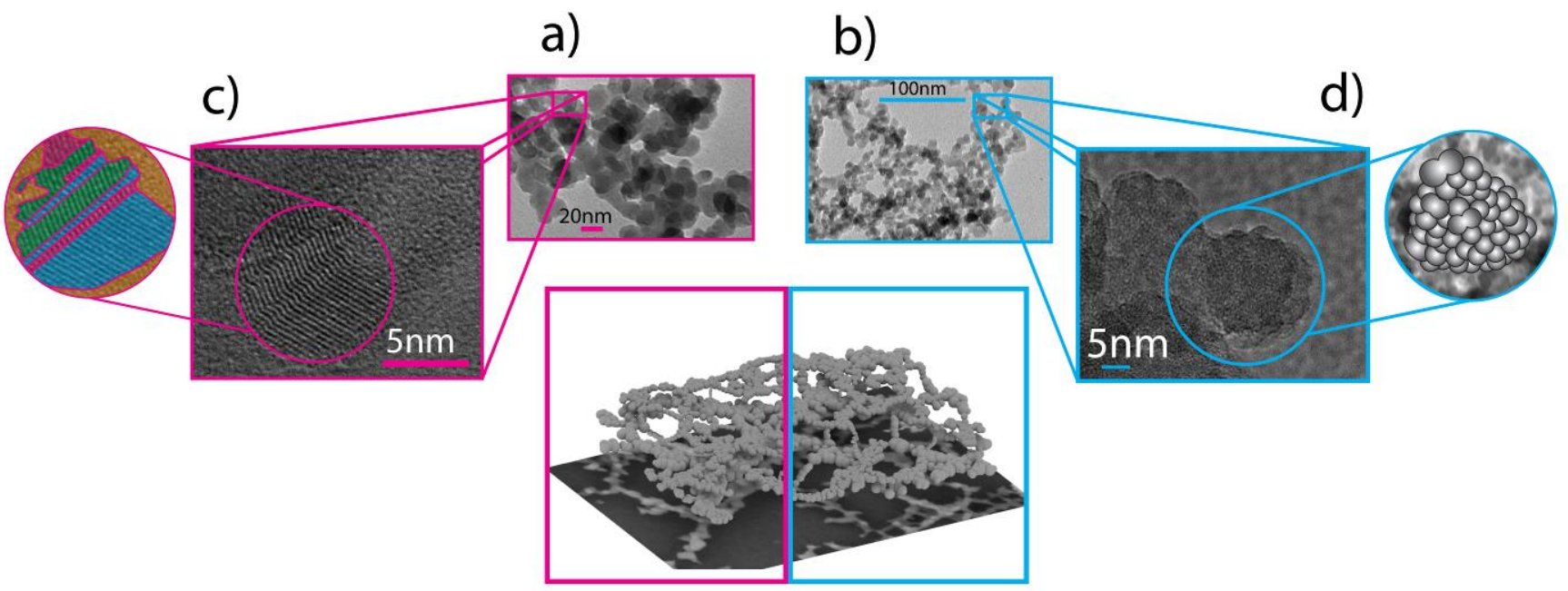

Figure 5-5: TEM and HRTEM images of disordered Si nanomesh structures formed under a) inert ion-plume conditions, b) oxygenated ion-plume conditions and the observed quantum scale defects c) sub-nanograin disorder within the NO structure and d) sub-nanovoids on NO surface

This disordered internal near quantum-scale grain boundary structure and sub-nanovoids surface morphology are to our knowledge not feasible with other fabrication techniques due to the nature 
by which the NOs form within the plume. The chaotic movement of ions within the laser-ion plume and formation of nanoclusters with varied crystal orientations, leads to the generation of disorders observed in Figure 5-3. The engineering of these defects within the NOs is only possible due to the breakdown of a sc-Si wafer through ionization and self-assembly within the ion-plume. Other fabrication techniques used to form Si nanostructures for SERS applications produce reported scSi nanostructures though lithographic ${ }^{[5-39]}$ or etching techniques. ${ }^{[5-40,5-41]}$ However there has yet to be an investigation into the role of sub-nano defects in Si nanostructures play in Raman activity due to the common self-assembly techniques inability to engineer quantum defects within nanostructures beyond point defects or doping.

An additional analysis of the size/size distribution of NOs within the nanomesh structures shows that the NO size and distribution is affected by the laser ionization energy and the conditions of the ion-plume. Higher ionization energy under both inert and oxygenated ion-plume conditions yields NOs sizes with a broader size distribution than NOs formed at lower ionization energy. However, the size/size distribution of NOs formed under inert conditions span a broader distribution than those formed under oxygenated conditions. The HRTEM images reveal that the NOs and the disorders within the NOs approach the quantum scale regime (defined as several nanometers in size ${ }^{[5-42,5-43]}$ ) These images also reveal that the presence of these sub-nano defects is more prevalent in NOs formed in an inert ion plume at higher ionization energy.

\subsubsection{Sub-nanograin Disorder}

An analysis of the XRD spectra (Figure 5-3b, 5-4b) obtained from the nanomeshes reveals that, the ion-plume conditions produce crystal grain orientations of $\mathrm{Si}$ depending on the plume conditions and ionization energy. The XRD plots show that the nanomesh structures created in this study are composed of nanocrystalline Si ([111], [220] and [311]) and a portion of amorphous $\mathrm{Si} / \mathrm{SiO}_{2}$. There is an observed peak when $2 \theta \sim 32^{\circ}$, which is an artifact of the XRD spectrometer, but occurs as a forbidden peak of [100] $\mathrm{Si}^{[5-44]}$ The determination of the crystallite size reveals the quantum scale nature of the nanograin disorder observed in the NO formed under inert ion-plume conditions. The crystallite size can be determined by the width of the obtained XRD peaks. To determine an estimate of the crystallite size, the Scherrer equation ${ }^{[5-45]}$ has been used.

$$
\tau=\frac{K \lambda}{\beta \cos \theta} \quad \text { (Equation 5-2) }
$$


Where $K$ is a dimensionless shape factor that varies with the shape of the crystallite, for this study it is assumed that the shape factor is 0.9 assuming that the crystallites are spherical. $\lambda$ is the wavelength used to obtained the XRD spectra, in our case it is $0.154 \mathrm{~nm}$. $\beta$ is the measured peak broadening width at full width half maximum (FWHM). $\theta$ is the Bragg angle for the desired peak. The crystallite size is assumed to be the size of the individual regions of crystallinity within an individual NO. This is because the individual NOs are measured to have sizes on the order of $\sim 10 \mathrm{~nm}$, and the observed crystallite sizes are on the order of a few nanometers. This indicates that each individual NO is comprised of multiple quantum scale crystallites each having a different crystal orientation. It has been observed in Figure 3a, that the concentration of these crystallites within the NOs can be manipulated by altering the ionization conditions. Under inert ion-plume conditions, the [111] and [220] orientations of Si remain relatively unchanged, but the [311] orientation is not observed under these conditions and becomes significantly large under oxygenated ion-plume conditions at lower $\alpha$. At higher $\alpha$, vary slightly compared to lower $\alpha$ values, with [220] becoming significantly larger under both suppression and enrichment conditions.

These NO size/size distribution measurements coupled with the calculation of crystallites reveal that as ionization energy increases, the size distribution of NOs increases and the calculated internal stress within the NO increases and additional crystal orientations of Si become observable. This indicates that as the ionization energy of the laser pulses increase, more NOs of larger size are generated that have more variety of crystallites. This indicates that more sub-nanograin boundaries are present within the NOs generated at high ionization energy than at low ionization energy and result in a higher residual stress within the NO and a greater concentration of grain boundaries within the NO structure. This result shows that ionization energy plays a direct role in the grain boundary disorder of the generated NOs.

The Raman spectra of with these nanomesh structures give key morphological characteristics of the NO sizes and the stress present within the nanostructures.

The residual stress within the disordered nanomeshes can be identified and calculated by determining the shift in the positions of Raman peaks associated with Si. Residual stress in a crystal structure can manifest as a positive or negative in the peak position depending on the type of stress present in the nanostructure, either compressive or tensile respectively ${ }^{[5-46]}$.

To calculate the residual stress in the nanostructures, the following equation is used: 


$$
\sigma(M P a)=-4.35 M P a \times\left(\omega-\omega_{0}\right)(\text { Equation 5-3) }
$$

Where $\sigma$ is the calculated stress in $\mathrm{MPa}, \omega_{0}$ is the observed peak position of the $\mathrm{Si}$ wafer substrate and $\omega$ is the observed peak shift of the nanomesh structures. The value 4.35 MPa corresponds to the stress sensitivity of silicon. Figures 5-3e shows plots of these values for each nanomesh. Figure 5-3e, illustrates that the sub-nanograin disorder within the nanomesh is significantly higher at high ionization energy than at low ionization energy. This substantial increase in the stress in confirmed by the HRTEM images in Figure 5-3; a higher density of sub-nano grains are observed in the NO structure than in NOs formed at lower ionization energy.

Each of the above factors, quantum scale grain defects, increased residual stress and NO size contribute to the observed Raman activity in specific ways that have cooperative effects. It is theorized that the increased particle size distribution of the nc-Si NOs and the quantum-scale crystallite size results in more large NOs with smaller crystallites. Previous research has shown that a similar pattern of $\mathrm{EF}$ decrease as crystallite size increases for crystalline $\mathrm{Si}^{[5-47]}$. This increased density of crystallites within individual NOs, induces more residual stress in the NOs, resulting in both a larger concentration of grain boundaries within the NOs and more uncoordinated defect sites. These multiplicative factors working simultaneously result in the observed enhancement of the Raman signal from the nc-Si nanomeshs.

\subsubsection{Surface Nanovoid disorder}

An evaluation of the surface nanovoids produced under oxygenated ion-plume conditions presents complications due to the very small scale of the sub-nanovoids on the NOs surface. As observed from the HRTEM images, the nanovoids are less than $5 \mathrm{~nm}$ in scale, and as such quantifying the nanovoid density from these nanovoids is beyond this present study. A qualitative investigation however is useful for illustrating the observed results. The HRTEM images in Figure 5-5d show that at high ionization energy, the nanovoids are significantly dense on the surface of the NOs. The nanovoids observed on the NOs formed at low ionization energy tend to be broader and less condensed on the NO surface. A more in-depth analysis of the nanovoid concentration and its role in nanobiosensing is an important topic for a future study. The TEM images reveal that the NOs formed under oxygenated ion-plume conditions, similar to those formed under inert ion-plume conditions, are within the defined quantum scale regime, $\sim 10 \mathrm{~nm}$ for both high and low ionization energies. Since the HRTEM images did not reveal observable nanograins, an evaluation of the 
crystallite size for these nanomesh structures was not performed. This lack of observable crystallites is confirmed though calculation of the residual stress within the NOs; the calculated stress is approximately constant at both high and low ionization energies and remains lower than the stress observed in the disordered nanograin nanomeshes.

\subsubsection{SERS Biosensing Activity of Nanomesh}

The Raman active behavior of the disordered a/nc-Si nanomeshes were examined to determine the Raman activity effects of the observed disorder present within each nanomesh. In order to determine how these types of disorders effect Raman activity at different wavelengths, $785 \mathrm{~nm}$ and $532 \mathrm{~nm}$. The Raman spectra of the a/nc-Si nanomeshes are shown in Figure 5-6. The spectra in Figure 5-6 are evaluated by the observed peak at $520 \mathrm{~cm}^{-1}$ which is due to the LO phonon mode associated with crystalline $\mathrm{Si}^{[5-47]}$.
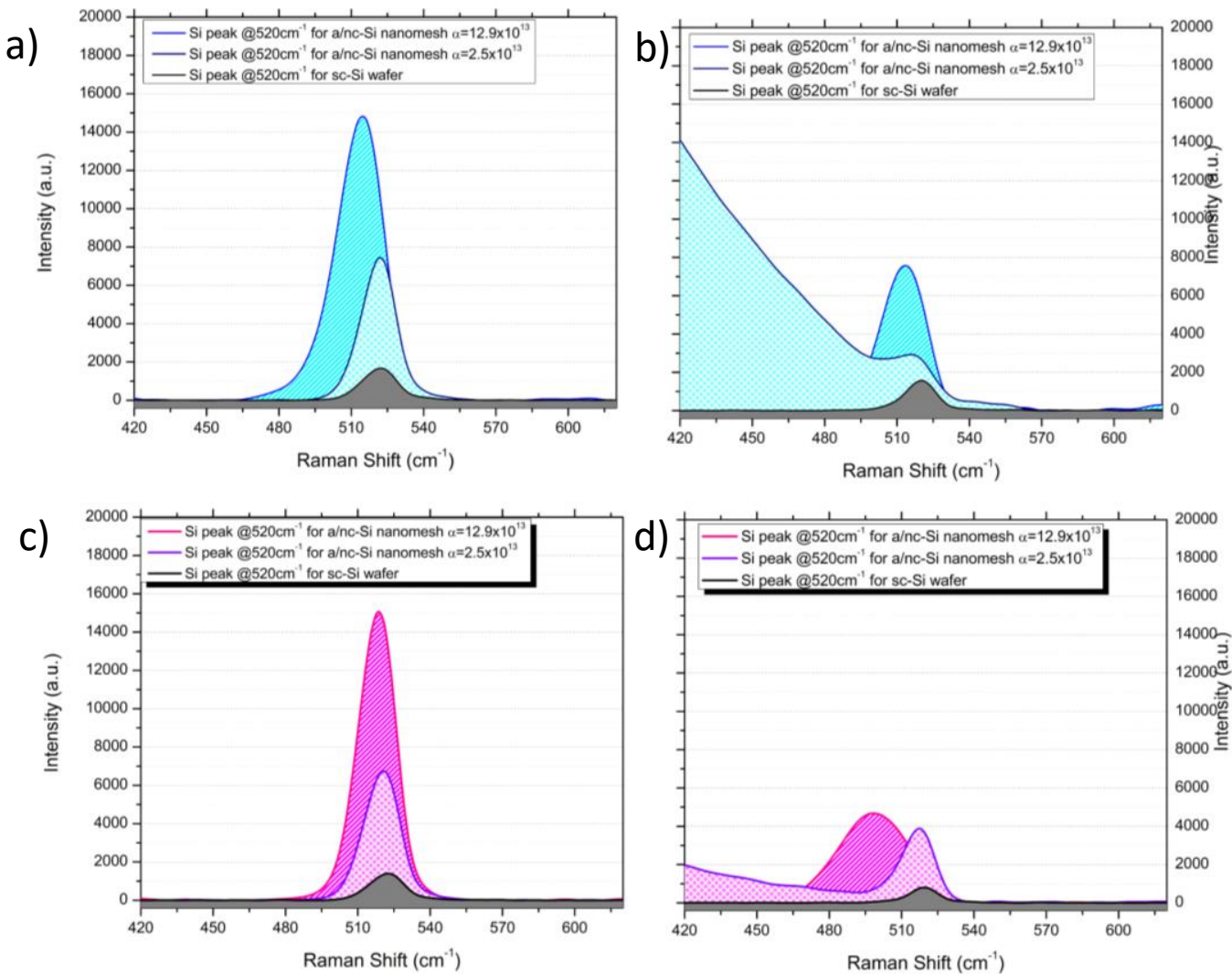

Figure 5-6: Raman spectra of the a/nc-Si nanomesh a) under oxygenated ion-plume conditions @785nm, b) oxygenated ion-plume conditions @ 532nm, c) inert ion-plume conditions @ 785nm and, d) inert ion-plume conditions @785nm 
The spectra in Figure 5-6 show that the Raman activity of the nanomesh structures is highly dependent both the type of quantum-scale disorder within the nanomeshes are formed and the wavelength of the Raman laser used to acquire the spectra. These spectra reveal that the type of disorder present within the nanomesh structures plays a significant role in the Raman activity of the nanomesh and the Raman activity caused by the sub-nano disorder is wavelength dependent. The Raman activity of these nanomeshes are quantified in Figure 5-7 using Equation 5-3

$$
E F_{\text {nanomesh }}=\frac{I_{\text {nanomesh }}}{I_{\text {wafer }}} \quad(\text { Equation 5-4) }
$$

Where $I_{\text {nanomesh }}$ and $I_{\text {wafer }}$ are the intensities of the $520 \mathrm{~cm}^{-1}$ from the a/nc-Si nanomesh structures and the sc-Si wafer substrate respectively.
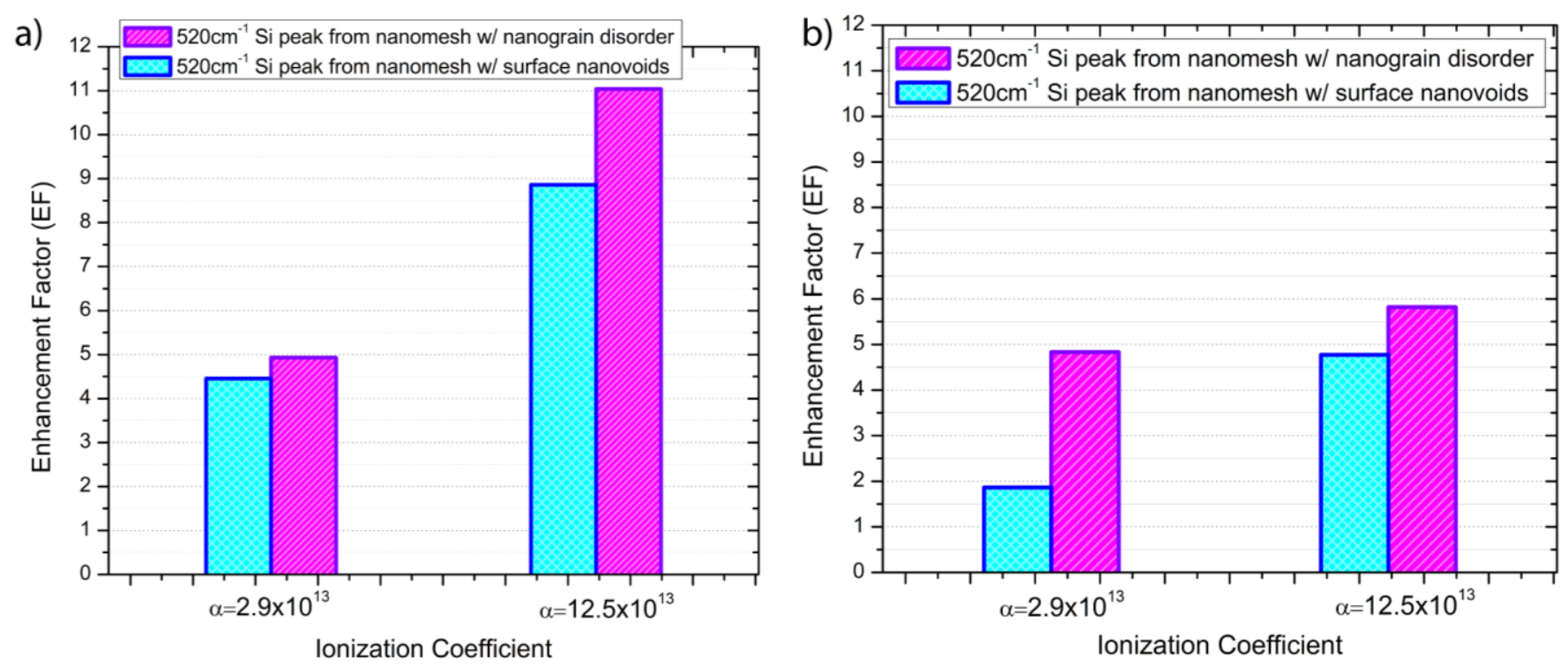

Figure 5-7: $\mathrm{EF}_{\text {nanomesh }}$ for nanograin disorder (pink) and surface nanovoid (blue) nanomeshes at

a) $785 \mathrm{~nm}$ and b) $532 \mathrm{~nm}$

The EF values show that the nanograin nanomeshes have a notable larger Raman activity compared to the nanovoid nanomeshes for both $785 \mathrm{~nm}$ and $532 \mathrm{~nm}$ wavelengths. This indicates that the sub-nano disorder in the inert nanomeshes (nanograin boundary disorder) exhibits larger Raman activity than the sub-nano disorder observed in the oxygenated nanomeshes (surface disorder). This suggests that the increased grain boundary disordered is causing an increase in the 
vibrational activity of the LO phonon mode of within Si that is responsible for the $520 \mathrm{~cm}^{-1}$ peak [47]

However, these spectra do show consistency regardless of conditions and wavelength of laser used; the Si peak from the sc-Si wafer is always than the peak from the nanomeshes, and the $520 \mathrm{~cm}^{-1}$ peaks from the nanomeshes formed at lower ionization energy are lower than the peaks from nanomeshes formed at high ionization energy. These observations demonstrate that the nanomeshes are significantly Raman active compared to the sc-Si wafer substrate and that the higher ionization produces higher Raman activity from the nanomesh structures than the lower ionization energy nanomeshes for both Raman wavelengths.

\subsubsection{Bioanalyte Enhancement efficacy of quantum scale disordered nanomeshes}

The SERS enhancement behavior of the quantum scale disordered nanomeshes are examined using a biomolecule, l-glutathione (GSH). This GSH biomolecule is classified as a tripeptide biomarker, which is an abundant intracellular biomolecule whose metabolism has been linked to liver disease, cancer growth, and neurodegenerative diseases. ${ }^{[5-48]}$ The detection of GSH at low concentrations using SERS biosensing techniques could potentially allow for the early detection of these diseases. GSH is also known to have very low vibrational activity as evidenced by low enhancement factors observed for GSH detection. ${ }^{[5-49]}$ The SERS enhancement capability of the disordered Si nanomeshes are evaluated in this study by measuring the Raman response of GSH in the presence of the nanomeshes at both $785 \mathrm{~nm}$ and $532 \mathrm{~nm}$ wavelengths to determine how the observed quantum scale disorder differences in Raman activity of the nanomeshes play a role in GSH detection.

To determine how effective the a/nc-Si nanomeshes are at enhancing the biomarker molecule $\mathrm{GSH}$, the EF values for the respective nanomesh structures are calculated. To calculate the EF values for the GSH biomolecule, the following equation was used:

$$
E F=\frac{I_{\text {nanomesh }} / N_{\text {nanomesh }}}{I_{\text {wafer }} / N_{\text {wafer }}} \quad(\text { Equation } 5-5)
$$

Where $I_{\text {nanomesh }}$ is the intensity of the identified characteristic for GSH on the nanomesh structure and $I_{\text {wafer }}$ is the intensity of this same peak on the Si wafer substrate. $N_{\text {nanomesh }}$ is an estimation of the number of GSH molecules on the nanomesh structures contributing to the $I_{\text {nanomesh }}$ value and 
$N_{\text {wafer }}$ is an estimation of the number of molecules on the Si wafer substrate contributing to the Iwafer value.

Figure 5-8 shows selected Raman spectral data and calculated EF values for the detection of GSH with Si nanomesh structures with sub-nano grain disorder.
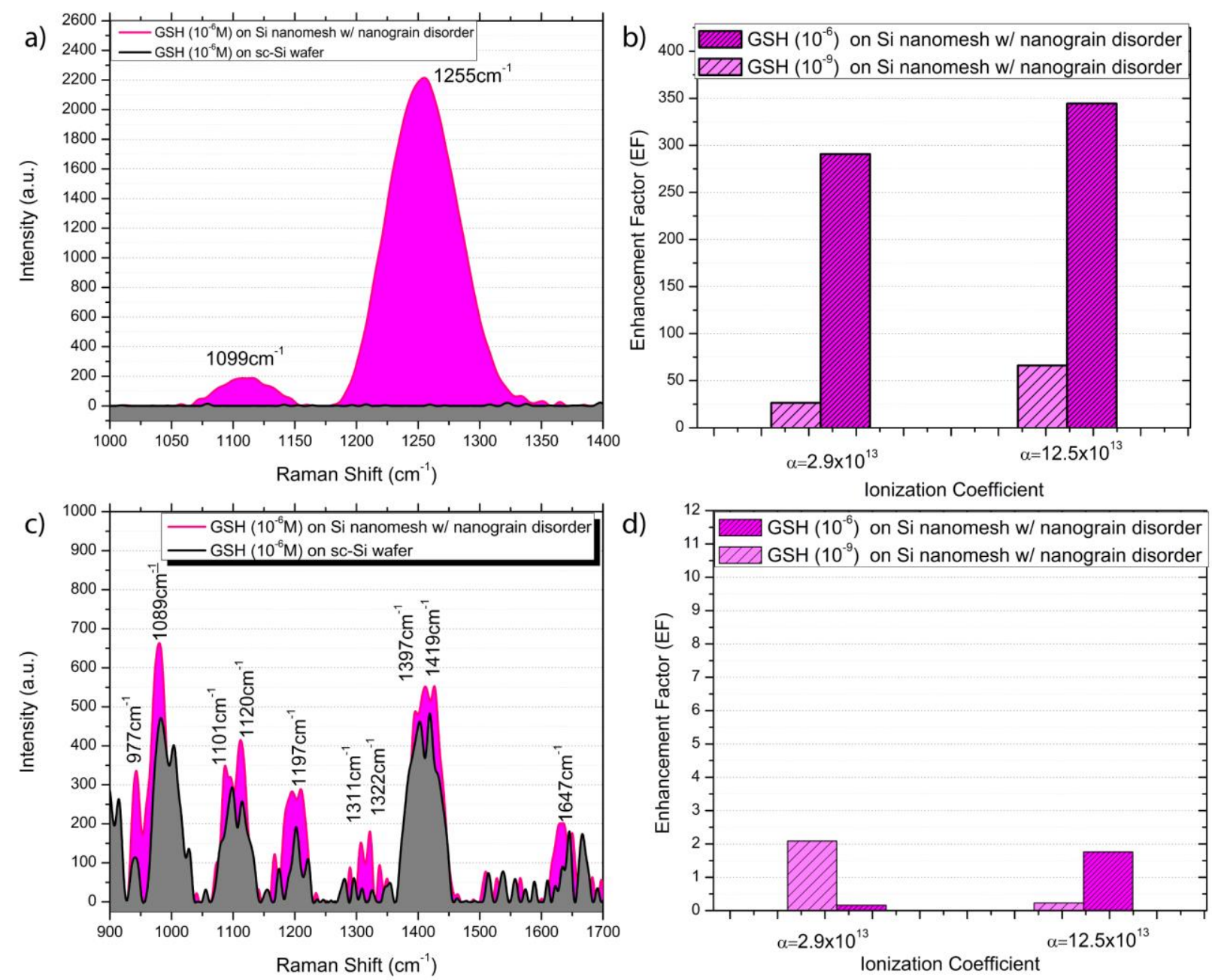

Figure 5-8: a) Raman spectra of GSH peaks @ 785nm, b) calculated EF values for the $1255 \mathrm{~cm}^{-1}$ peak @785nm wavelength, C) Raman spectra of GSH peaks @532nm, d) calculated EF values for the $1419 \mathrm{~cm}^{-1}$ peak @ 532nm wavelength

The spectra in Figure 5-8 show SERS detection of the GSH biomolecule in the presence of the nanomesh structures at both $532 \mathrm{~nm}$ and $785 \mathrm{~nm}$ Raman laser wavelengths. This indicates that these 
nanomeshes are activating distinct vibrational modes of the GSH molecule and demonstrates that the 3D nanomesh morphology and quantum-scale disorder dictates the SERS enhancement of the GSH and varies based on the Raman laser wavelength. The observed peaks of GSH on the nanomesh structures are displayed in Table 5-1 along with peaks assignments for the peaks corresponding to literature. In Figure 5-8, it can be seen that the nanomesh enhances different vibrational modes depending the wavelength of Raman laser and as such the chosen characteristic peak differs between $532 \mathrm{~nm}$ spectra and $785 \mathrm{~nm}$ spectra. For this reason, the $1419 \mathrm{~cm}^{-1}$ for $532 \mathrm{~nm}$ spectra and the $1255 \mathrm{~cm}^{-1}$ peak for $785 \mathrm{~nm}$ spectra were chosen respectively. These observed peaks for GSH are in good agreement with the literature. ${ }^{[5-34,5-50,5-51]}$ For SERS detection of GSH with NMNs, Lee et.al ${ }^{[5-52]}$ have observed three GSH peaks at $10 \mu \mathrm{m}$ concentration and Huang et.al ${ }^{[5-49]}$ have observed nine peaks of GSH at $10 \mu \mathrm{m}$ concentration for Au nanoparticles on Si nanospheres coated with a Ag nanofilm and Ag nanoparticles in colloidal solution, respectively. Ten major peaks are observed for GSH at $1 \mu \mathrm{m}$ concentration at $532 \mathrm{~nm}$ wavelength and two major peaks for GSH at $1 \mu \mathrm{m}$ concentration at $785 \mathrm{~nm}$ wavelength. As a result, the Si nanomesh structures are able to activate a comparable number of vibrational modes of the GSH molecule without plasmonic nanostructures.

Table 5-1: Observed Peaks for GSH on nanomeshes with peak assignments from literature ${ }^{[5-34]}$

\begin{tabular}{|c|c|c|c|c|c|}
\hline $\begin{array}{l}\text { GSH observed } \\
\text { peaks @785nm }\end{array}$ & $\begin{array}{l}\text { GSH peaks from } \\
\text { References }\end{array}$ & $\begin{array}{l}\text { Peak } \\
\text { Assignment }\end{array}$ & $\begin{array}{l}\text { GSH Observed } \\
\text { Peaks@ @532nm }\end{array}$ & $\begin{array}{l}\text { GSH peaks from } \\
\text { References }\end{array}$ & $\begin{array}{l}\text { Peak } \\
\text { Assignment }\end{array}$ \\
\hline 1099 & 1074 & $\mathrm{C} 12 \mathrm{H} 2 \mathrm{wag}$ & 977 & 972 & CC12 wag \\
\hline \multirow[t]{9}{*}{1255} & 1255 & $\mathrm{C} 12 \mathrm{H} 2$ twist & 1089 & 1074 & C6C7 stretch \\
\hline & & & 1101 & 1097 & NC17 stretch \\
\hline & & & 1120 & 1117 & $\mathrm{C} 12 \mathrm{H} 2$ wag \\
\hline & & & 1197 & 1169 & $\mathrm{C} 12 \mathrm{H} 2$ twist \\
\hline & & & 1311 & 1309 & $\mathrm{C} 17 \mathrm{H} 2$ wag \\
\hline & & & 1322 & 1334 & $\mathrm{C} 17 \mathrm{H} 2$ wag \\
\hline & & & 1397 & 1403 & $\begin{array}{l}\mathrm{CO} 2 \text { symmetric } \\
\text { stretch }\end{array}$ \\
\hline & & & 1419 & 1415 & C6H2 wag \\
\hline & & & 1647 & 1660 & C8O stretch \\
\hline
\end{tabular}


Figures 5-8b,d show a similar trend to the EF values observed in Figure 5-7, the nanomesh composition and morphology play a pivotal role in the SERS activity of the nanomesh and the enhancement of the GSH spectral peaks. These plots demonstrate that the disordered nanomesh structures are able to achieve a significant enhancement of the GSH biomolecule on the order of $4 \times 10^{2}$. The results coincide with the observations of the Raman activity of the nanomeshes; quantum-scale disordered nanomeshes achieve an observed enhancement across all ionization energies with the nanomeshes formed at the highest ionization energy demonstrating the largest observed enhancement. The EF values reveal that the extent to which the SERS enhancement of GSH with the nanomesh structures has a dependence on the wavelength of Raman laser.

In Figure 5-8a) the observed GSH EF values follow a similar trend observed for the EF from the nanostructures, the nanomesh with nanograin disorder produce a higher enhancement than the nanomeshes with surface nanovoids at both $10^{-6} \mathrm{M}$ and $10^{-9} \mathrm{M}$ concentrations. This effect is attributed to the near-quantum scale nanograin disorder within the individual NOs of the nanomesh structure. The grain boundaries acts as intense Raman scattering centres and through the CT resonance mechanism, the vibration activity of GSH is increased. The high density of nearquantum scale nanograins within the NOs causes this significant enhancement in GSH spectral response.

Figure 5-9 shows selected Raman spectra for GSH solutions $\left(10^{-6} \mathrm{M}\right.$ and $\left.10^{-9} \mathrm{M}\right)$ on disordered $\mathrm{Si}$ nanomesh structures with sub-nano surface nanovoids and the calculated EF values. 

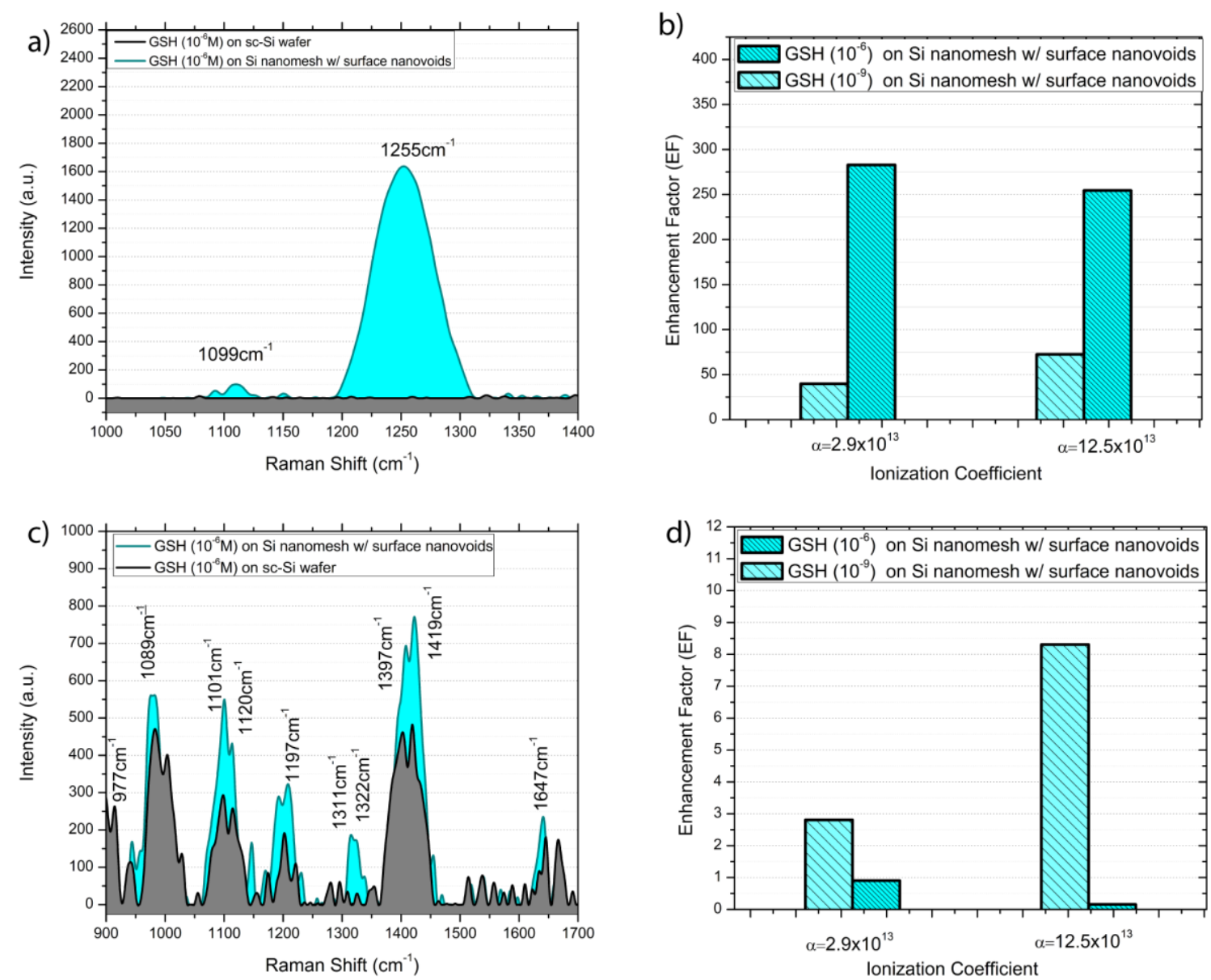

Figure 5-9: a) Raman spectra of GSH peaks @ 785nm, b) calculated EF values for the $1255 \mathrm{~cm}^{-1}$ peak @785nm wavelength, C) Raman spectra of GSH peaks @532nm, d) calculated EF values for the $1419 \mathrm{~cm}^{-1}$ peak @ 532nm wavelength

These spectra and calculated EF values show that the Si nanomesh structures with surface nanovoids are able to enhance the detection of the GSH analyte, and similar to the Si nanomeshes with nanograin disorder the SERS activity is highly dependent on the ionization energy of the ionplume and the Raman laser wavelength. The observed peaks of GSH on the nanomeshes with surface nanovoids coincide with those observed with peak observed from Figure 5-9

From the HRTEM images in Figure 5-4, the nanomesh structure with surface nanovoids have no observable nanograin structure leading to these structures relying on the nanovoids and highly $3 \mathrm{D}$ nanomesh structures as the primary SERS enhancing source. In Figure 5-9d, it is observed that 
the calculated EF is significantly higher at $10^{-9} \mathrm{M}$ concentration than at $10^{-6} \mathrm{M}$ concentration. It is proposed that this significant increase in the SERS enhancement of $10^{-9} \mathrm{M}$ GSH with the surface nanovoid nanomesh structure is due to a concept termed CT nanogaps. The nanovoids observed on the NO surface act as traps or wells for the analyte molecules. It is suggested that at this $10^{-9} \mathrm{M}$ GSH concentration, the GSH molecules when trapped within the nanovoids on the NO surface are more efficiently able to produce a CT resonance effect than compared to the $10^{-6} \mathrm{M}$ GSH solution.

A visualization of the nanograin disorder and surface nanovoid contributions to SERS detection of the GSH molecule is shown in Figure 5-10.
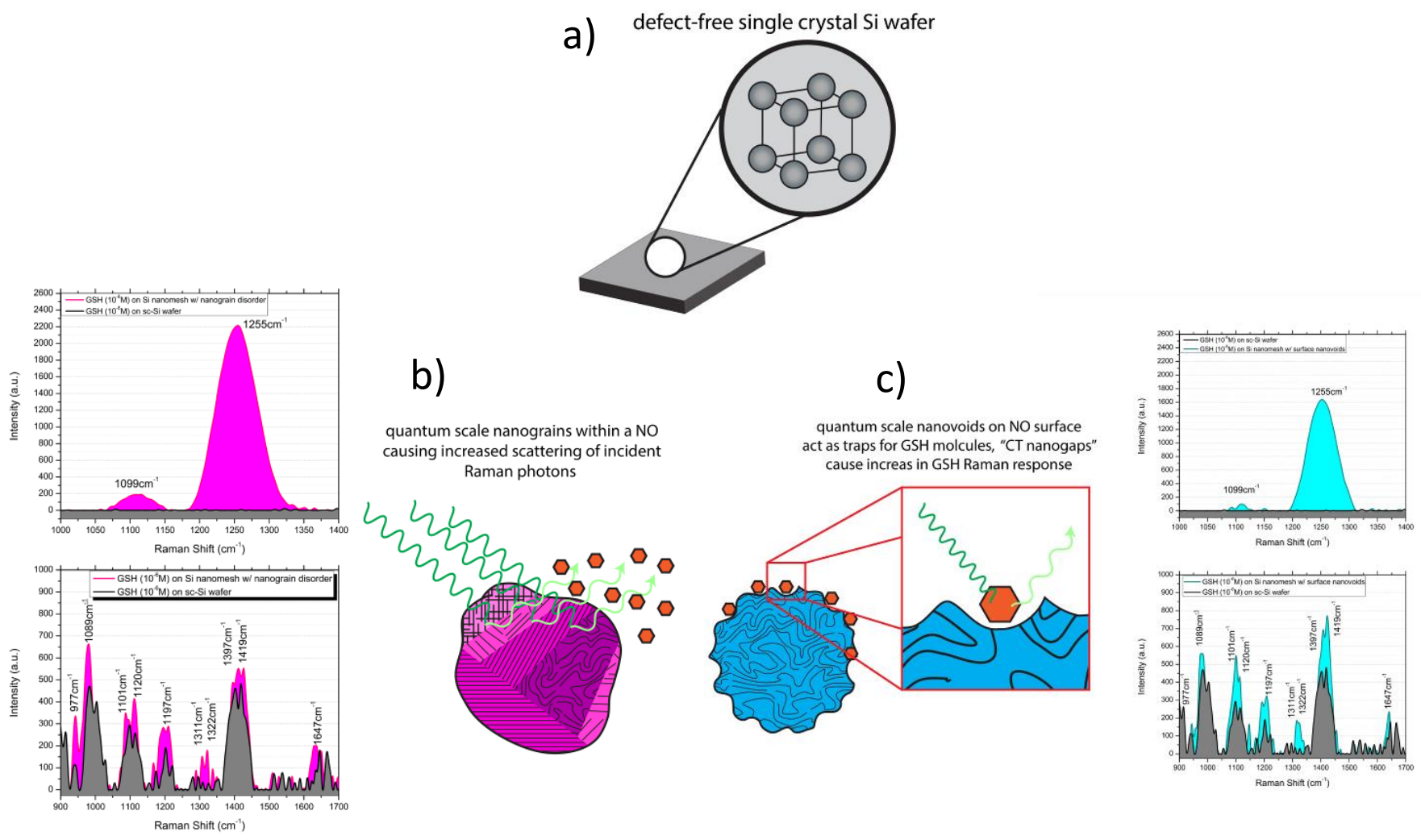

Figure 5-10: Visualization of the SERS enhancing effects caused by quantum scale defects, a) sub-nanograin boundary disorder defects and b) sub-nanovoids on NO surface

As discussed, the ionization energy has a significant effect on the SERS activity of the nanomesh structures and thus the detection of the GSH molecule. From the characterization results in Figures 5-3 and 5-4 it is posited in this study that the higher ionization energy under both inert and oxygenate ion-plume conditions yields a higher density of sub-nano disorder either nanograin disorder or surface nanovoids compared to the nanomeshes formed at lower ionization energy. 
From Figures 5-8 and 5-9 it can be seen that there is a variance in the SERS enhancement of GSH signal that is contingent upon which form of disorder is causing the SERS activity. The nanograin disorder achieves a higher EF for GSH at $785 \mathrm{~nm}$ than the surface nanovoids, yet the surface nanovoids are able to achieve a much higher EF for GSH at $10^{-9} \mathrm{M}$ concentration than the surface nanograin disorder. This variance in SERS activity of these quantum scale disorders that depends on the Raman laser wavelength can be applied to the detection of biomolecules in both in vivo and in vitro applications ${ }^{[5-53]}$. As such, the SERS enhancement can be tuned to detect biomolecules specific for applications by creating nanomeshes with specific quantum scale disorders.

\subsubsection{Towards Universal sensing with disordered Si nanomesh}

To determine the potential of the quantum scale disordered Si nanomesh structures as a universal SERS nanoprobe material, it is necessary to determine if the nanomesh structures are able to detect additional biomolecules beyond GSH. In this study, biomolecule analytes, tryptophan (Trp), cysteine (Cys) and methionine (Met) were tested for SERS response in the presence of the disorder Si nanomesh structures. These biomolecules were chosen because of their relevance as signalling molecules for diseases. Tryptophan was chosen due to its close relation to HIV infections and neurological disorders ${ }^{[5-54]}$. Cysteine was chosen due to heightened levels within the body being linked to cardiovascular diseases and neurotoxicity ${ }^{[5-55]}$. Methionine was chosen due to its role in determining liver function. ${ }^{[5-56]}$ These biomolecules are similar to GSH these biomolecules are known to have a very low Raman cross-section which makes them difficult to detect using SERS spectroscopy.

The results from the study of GSH have shown that the higher ionization energy yields quantum scale disorder that produce the largest enhancement. As such, for this study nanomeshes formed at higher ionization energy are used to enhance the spectra of the biomolecules. Additionally, the SERS spectra of the biomolecules were obtained using 532nm Raman laser wavelength because of the greater number of peaks which allows for more characteristic peaks foe the biomolecules to be distinguished. Figure 5-11 shows the SERS spectra of the Cys, Trp and Met biomolecules on the disordered nanomesh and the sc-Si wafer substrate. 

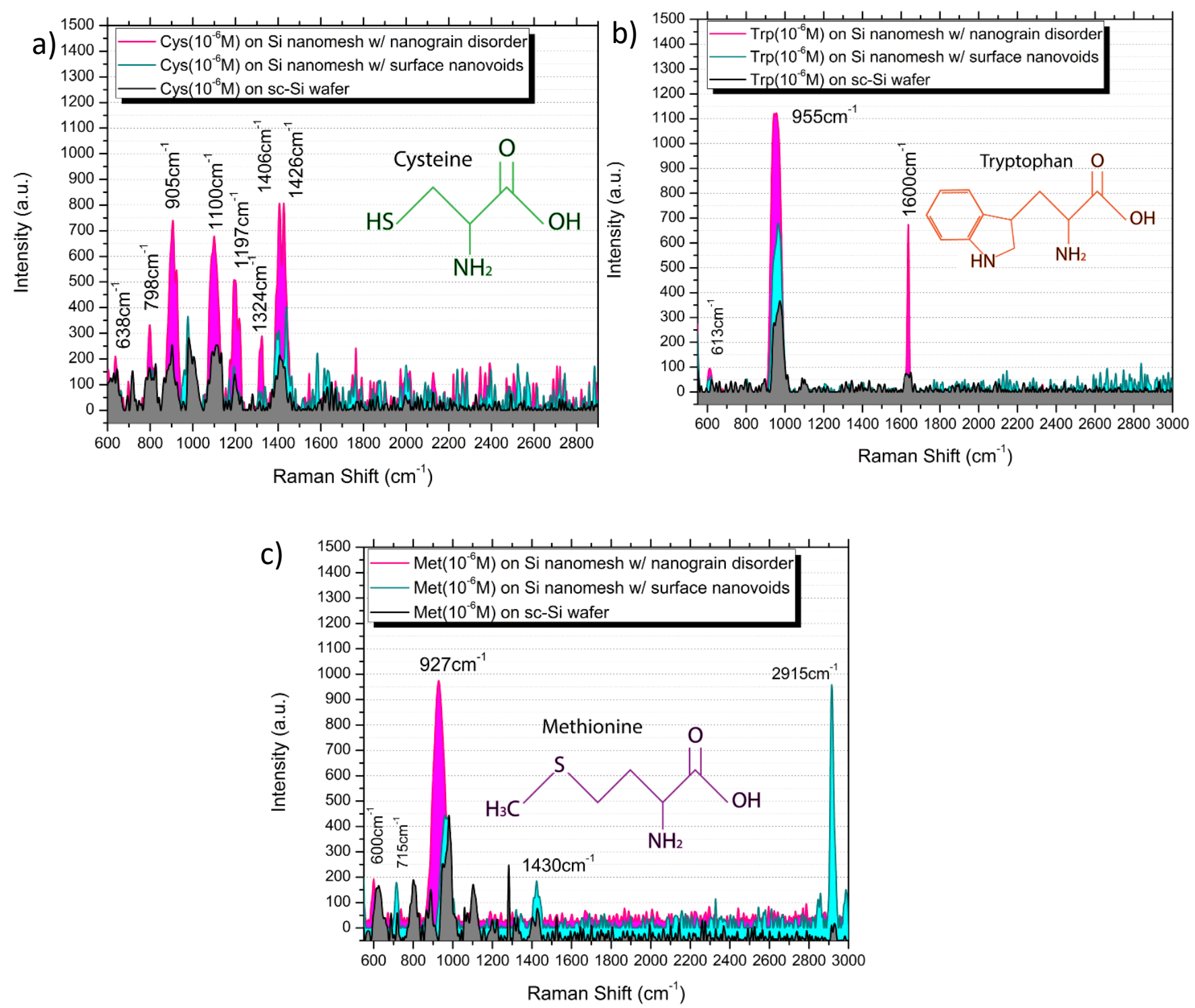

Figure 5-11: Raman spectra of a) Cys, b)Trp and c) Met on nanomeshes with (pink) subnanograin boundary defects, (blue) surface sub-nanovoids and on (grey) defect free sc-Si wafer substrates

These spectra show that these biomolecules also exhibit greatly enhanced spectra in the presence of the disordered nanomeshes. Both the nanomeshes with nanograin disorder and surface nanovoids are able to enhance the SERS spectra of Cys, Trp and Met. It is also observed that the type of disorder present within the nanomesh can activate the vibrational modes of a biomolecule that cannot be activated by the other type of disorder. For instance, the nanograin disorder is able to activate the benzene/pyrrole ring stretching mode of Trp while surface nanovoids cannot. 
Conversely, surface nanovoids can active the asymmetrical $\mathrm{CH}_{2}$ stretching mode of Met, while nanograin disorder cannot. The observed peaks for identified by these spectra are displayed in Tables 5-2-5-4.

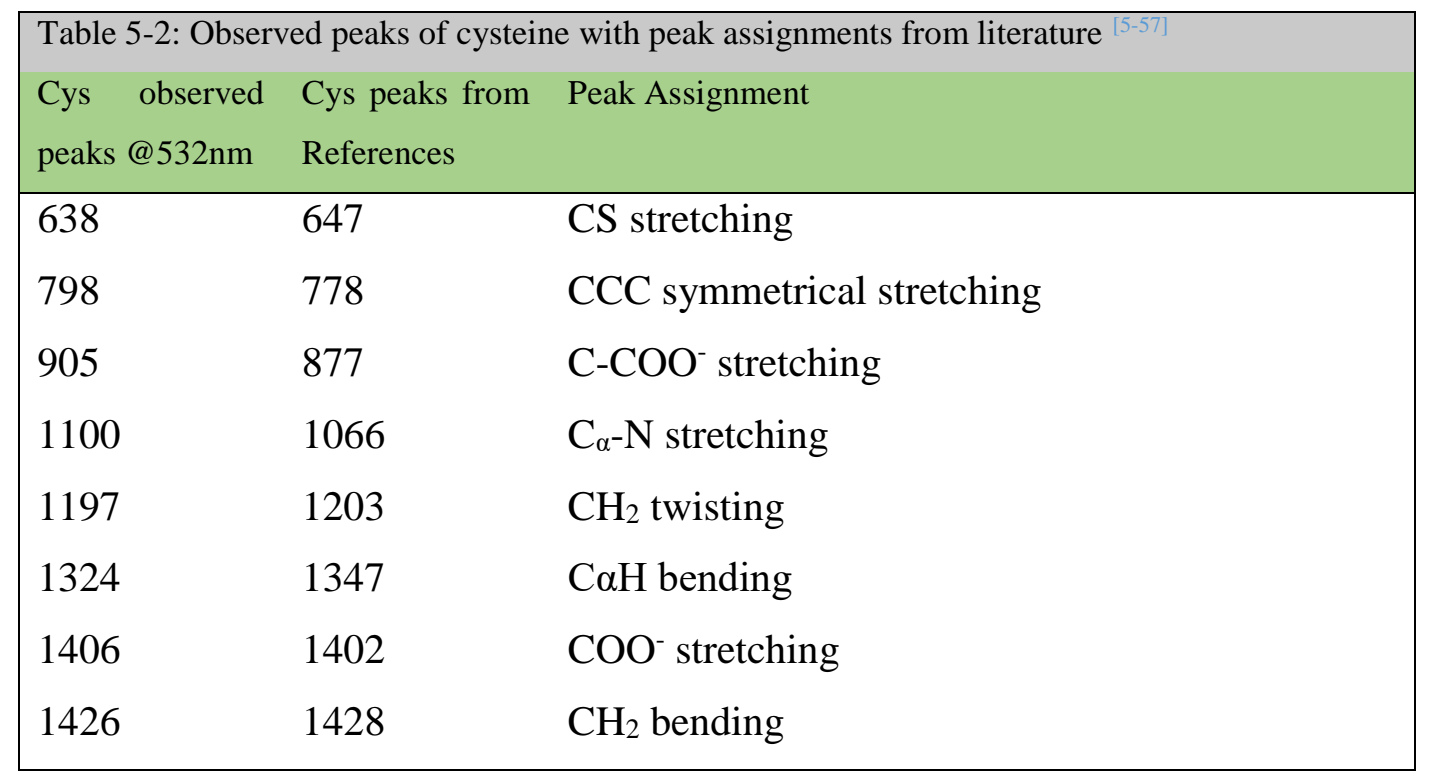

\begin{tabular}{|lll|}
\hline \multicolumn{4}{|l|}{$\begin{array}{l}\text { Table 5-3: Observed peaks of tryptophan with peak assignments from literature } \\
\text { Trp observed } \\
\text { peaks @ } \begin{array}{l}\text { Trp } 52 \mathrm{~nm} \text { peaks from } \\
\text { References }\end{array}\end{array}$} & Peak Assignment \\
\hline 613 & 596 & NH bending; deformation of benzene, pyrrole ring \\
955 & 974 & H twisting in benzene ring \\
1600 & 1608 & Benzene ring/pyrrole ring stretching \\
\hline
\end{tabular}

\begin{tabular}{|c|c|c|}
\hline $\begin{array}{l}\text { Met observed } \\
\text { peaks @ } 532 \mathrm{~nm}\end{array}$ & $\begin{array}{l}\text { Met peaks from } \\
\text { References }\end{array}$ & Peak Assignment \\
\hline 600 & 658 & C-S-C symmetrical stretching; $\mathrm{O}-\mathrm{C}=\mathrm{O}$ in-plane deformation \\
\hline 715 & 724 & C-S-C asymmetrical stretching \\
\hline 927 & 921 & O-H out-of-plane deformation; $\mathrm{C}$-C stretching \\
\hline 1430 & 1433 & $\mathrm{CH}_{3}$ asymmetrical deformation; $\mathrm{CH}_{2}$ symmetrical deformation \\
\hline 2915 & 2920 & $\mathrm{CH}_{2}$ asymmetrical stretching \\
\hline
\end{tabular}


The detection of these distinct biomolecules with both types of nanomesh disorder demonstrates the potential use of this unique Si nanostructure as a universal nanoprobe material that can be used for early detection of disease signals.

\subsection{Summary}

In this study it has been shown that the UFPL ionization mechanism can be reconditioned by influencing the $\mathrm{Si}-\mathrm{O}_{2}$ ion interactions to induce sub-nano disorder within individual hybrid a/ncSi NOs that comprise a 3D nanomesh structure. By either influencing the ions interactions to create an inert or oxygenated laser-ion plume, the ionization mechanism and the a/nc-Si NO formation mechanism can be tuned to generate distinct forms of quantum scale defects that manifest as increased sub-nanograin boundaries within NOs and as disordered nanovoids on the NO surface. It has been shown that these quantum scale defects can be tuned and by doing so changes in the Raman activity can be induced from the resultant a/nc-Si nanomesh structures. These nanomeshes also exhibit changes in SERS biosensitivity for the detection of a tripeptide biomolecule. Additionally, it has been shown that these quantum-scale defects can be employ within a 3D Si nanomesh structure for the potential use as a universal sensing nanoprobe for early stage disease signalling. This result establishes the possibility of being able to engineer quantum scale defects in Si nanostructures for advanced SERS nanobiosensing applications. 


\section{$\underline{\text { Chapter } 6}$}

\section{Conclusions and Recommendations for Future Research}

\subsection{Conclusions}

In this dissertation, the creation of a Si-based nanomaterial that exhibits ultrasensitive SERS activity for chemical and biosensing applications is presented. The unique structure and Raman enhancing characteristics of this Si nanomaterial are facilitated by using the femtosecond pulsed laser ionization mechanism

The main objective of this thesis was to activate the SERS enhancing characteristics of the Si nanomaterial and to gain a fundamental understanding of how the laser ion-plume formation mechanism can be manipulated to produce a highly SERS active platform from Raman insensitive material. The use of this fabrication technique and the creation of this Si nanostructure for SERS detection has yet to be reported in the field of semiconductor SERS research; the field of Si-based SERS nanostructures is a research area in the nascent stages of development, with very few current studies being conducted. When comparing these $\mathrm{Si}$ nanostructures to other SERS semiconductor/metal oxide nanostructures, the level of enhancement is often comparable to the enhancement reported by these nanostructures and in some cases exceeds the reported SERS enhancement values.

The fabrication of a self-assembled three-dimensional interlinked nanoweb network through laser ionization is presented. The ionization of the single crystal Si wafer substrate and the reformation of the $\mathrm{Si}$ ions within the ion-plume yields individual nanospheroids that are comprised of crystalline grains of $\mathrm{Si}$ that have multiple orientations and amorphous silicon. These hybrid Si nanospheroids that become interlinked and fused upon self-assembly on the Si wafer surface form a highly three-dimensional and complex nanoweb structure.

This hybrid Si nanostructure exhibited exceptional SERS activity for the detection of chemical analytes R6G and CV through charge-transfer resonance and are able to produce an enhancement of these analytes comparable to those achieved by noble metal nanostructures, which are regarded as the standard for producing enhanced SERS signals through surface plasmon resonance. 
By surface modifying the highly sensitive Si nanostructures with a layer of gold nanoparticles, a two-fold contribution to SERS enhancement of R6G and CV was observed. This combination of charge-transfer enhancement and surface plasmon resonance mechanisms is rarely considered with other nanostructure forms that solely rely on noble metals as the SERS enhancing sources.

With further refinement of the primary Si nanospheroids as a SERS booster and NMNs as a secondary boosting source, it was found that the layer thickness of NMN nanoparticles, the size of the NMNs and their composition can be modulated to achieve a significant increase in the detection of a biomolecule analyte, L-glutathione, which is known for low Raman vibrational activity.

In an effort to further delve into the fundamental principles of how Si can be used as a SERS active sensing material, quantum-scale defects are introduced into the Si nanospheroid structure by amending the ion-interactions within the laser-ion plume. The results show that the SERS enhancement character of the Si nanostructures is highly dependent on the defect structure and scale along with the near quantum scale morphology of the 3D nanoweb structures. The SERS detection of multiple biomolecular disease signal molecules with these quantum scale defects structures, establishes the possibility of Si-only nanostructures to be used as universal disease detection nanoprobe materials. 


\subsection{Future Research}

With the development of these Si-based nanostructures that have substantially high SERS enhancement, the possibility of further developing Si based nanomaterials is immense and the enrichment of this enhancement mechanism with additional SERS active nanomaterials is an extremely interesting research avenue. The following future objectives represent potential advancements that can be made using the laser-ion plume mechanism for Si-based SERS nanostructures

6.2.1. Au-Si alloy-like nanostructure formation by laser ionization for dual SPR-CT SERS biomolecule enhancement

In this thesis, the SERS enhancement effects of Si nanostructures and boosting these SERS effects with additional external noble metal nanostructures for both chemical and biosensing applications have been studied. By using the unique laser ionization nanostructure formation mechanism, previous studies have demonstrated that by ionizing a gold-coated Si wafer substrate under the right conditions, a miscible Au-Si alloy-like nanostructure can be fabricated. In this proposed study, the SERS enhancing effects of internalized noble metal nanostructure within the hybrid Si nanostructures will be studied and how this alloy-like nanostructure can enhance the detection of bioanalytes will be studied. Some preliminary experimentation and analysis has been achieved and is presented here.

In this study the formation of a novel hybrid gold silicon alloy-like nanomaterial that exhibits activity of surface enhanced Raman scattering (SERS) is presented. This alloy-like material is distinctive from other nanomaterials due to the gold-silicon alloy-like nanospheroids are unable to be created using conventional fabrication techniques; it is reported that the use of a gold-silicon based material as the primary source of Raman enhancement. This interconnected nanospheroid network, formed with a femtosecond laser synthesis which enables the laser ion plume condensation formation mechanism, can be modulated in terms of material composition and morphological structure to maximize enhancing character of the nanostructure substrate. The combination of amorphous/crystalline silicon with embedded regions of gold in individual nanospheroids and the high Raman hotspot concentration lead to a highly Raman active nanostructure. 
There exists a current movement in literature to marry the SERS enhancement effects by developing hybrid nanostructures that have both a SPR contribution from NMNs and a plasmonic/charge-transfer contribution from a SERS active semiconductor/dielectric nanomaterial. For example Dai et.al have developed a $\mathrm{TiO}_{2} / \mathrm{Ag}$ hybrid nanostructure for detecting the presence of thiram, a toxic pesticide. The $\mathrm{TiO}_{2}$ nanosheets formed through hydrothermal synthesis are seeded with Ag nanoparticles and these particles are grown through an annealing process. With this hybrid nanostructure they have been able to detect thiram in both laboratory prepared and real samples with an observed limit of detection (LOD) of $\sim 10^{-7} \mathrm{M}$. Additionally Zhang et.al have created graphene oxide wrapped Ag nanoparticles for detecting PCBs, environmental organic pollutants. These Ag nanoparticles formed by chemical synthesis are coated with graphene oxide by an additional chemical process. They have observed a LOD of $10^{-4} \mathrm{M}$ for the PCB pollutant.

With these current results it is observed that a new phenomenon of SERS enhancement using a unique alloy-like material formed between silicon and gold which allows for a highly Raman active material. In this study, using an ultrafast pulsed laser formation mechanism, a synthesized a nanoweb material comprised of interconnected silicon nanospheroids which have localized regions of gold within each individual nanospheroid. This encasement of gold nanoclusters within the silicon nanospheroids gives this material a unique character which to our knowledge is unreproducible using other fabrication techniques; as silicon and gold are immiscible under ambient fabrication conditions. By altering the laser-ion plume interactions, programming the amount of gold within the silicon nanospheroids, the size of the gold nanoclusters and the physical morphology of the nanoweb structure itself is possible; each of these characteristics has a significant effect on the amount of Raman scattering and thus enhancement of a chemical applied to the nanostructures. The unique laser ion plume formation mechanism allows for the creation nanospheroids that have amorphous/polycrystalline hybrid silicon structure, and nanoclusters of gold encased within each nanospheroid. This material structure allows for a high concentration of grain boundaries, both between the amorphous and polycrystalline grains of silicon and between the amorphous/crystalline silicon grains and gold nanoclusters. This larger concentration of grain boundaries leads to a higher number of Raman scattering events. Figure 6-1 is a schematic showing the ionization of an Au coated Si wafer. 
The goal with this research is to demonstrate the ability to create a new silicon-gold hybrid nanomaterial, which has unique and heightened SERS properties which can be precisely manipulated by altering the laser-ion plume formation mechanism. Determining how the size and distribution of the gold nanoclusters have an effect on the Raman enhancement capability of the nanoweb structures as well as how ratio of gold to hybrid Si plays a role in Raman enhancement is of particular interest. In addition, the evaluation of this hybrid nanomaterial's potential as a platform for biosensing applications is obtained by determining its capability to enhance the detection of a commonly used bio-label molecule, in the interest of applying this material for the detection of biomolecules.

In order to determine how SERS enhancement can be achieved with the Au-Si nanoweb structures, an evaluation of how the alloy-like structure is formed, how the ion plume formation mechanism can be tuned to modify the Au:Si ratio within the individual nanospheroids and how these material characteristics can be optimized to increase the enhancement factor (EF) and sensitivity of the alloy-like nanostructures to the chemical and biomolecule analytes is required.

The formation of this distinctive alloy-like material between silicon and gold is as-to-yet, to our knowledge, unable to be formed using conventional fabrication techniques due in part to the immiscibility of $\mathrm{Au}$ and $\mathrm{Si}$. It has been documented that gold has a very limited solubility in crystalline silicon, with reported solubility of $10^{15}$ atoms $/ \mathrm{cm}^{3}$ and an Au concentration of $1.3 \times 10^{-4}$ at $\%$ at $1200^{\circ} \mathrm{C}$. This level of solubility of gold in silicon is achieved through liquid melt techniques to form eutectic alloy between gold and silicon, an alloy forming process which is highly conventional and does not yield interesting nanostructured materials. In this study, while by definition, not a true alloy this nanomaterial exhibits a unique morphological character and highlyactive nano-Raman sensing activity. The nanomaterial that is observed is an interconnected network of fused nanospheroids which form a nanoweb structure and each individual nanospheroid is composed of a hybrid amorphous/crystalline silicon with encased gold nanoclusters. During the laser-ion plume formation mechanism, the laser ionizes the substrate to form an ion plume; the substrate used in this study is a silicon wafer coated with a thin 300nm layer of gold. As such, when the laser strikes the substrate surface, gold atoms ionize first followed by the silicon atoms underneath. The tuning of the laser interactions with the substrate will determine the composition of the Au-Si nanospheroids; the power of the laser pulses, the time that the laser dwells on a spot 
within the array of dots will determine the depth at which the laser removes material from the substrate and thus the ratio of silicon to gold in the resulting nanospheroids. At higher laser pulse power, the depth of laser ionization is increased leading to a higher percentage of silicon present in the nanoweb structure conversely, lower laser pulse power leads to a higher percentage of gold. There exists a threshold limit of laser power is required to ionize the gold layer on the substrate and due to the increased reflective nature of the gold layer, this threshold is higher than the power threshold limit for silicon which means that if it is possible to ionize the gold layer, the ionization of the silicon wafer underneath will occur as well. This results in an ion plume that inherently contains both gold and silicon ions, of which the ratio of these species is dependent on the power of the laser pulses.

Within the ion plume, silicon and gold ions condense to form protoclusters of amorphous, polycrystalline and crystalline gold. Gold will condense first due to the faster condensation rate compared to silicon which will result in clusters of gold surrounded by grains of both amorphous and nanocrystalline silicon forming an alloy-like hybrid nanospheroid. Due to the grain boundary separation between the gold clusters and the amorphous/polycrystalline silicon grains and the grain boundaries separating the amorphous and polycrystalline silicon grains, these nanospheroids have a high concentration of grain boundaries leading to unique Raman scattering characteristics.

The main objective of this study was to determine if this unique nanomaterial that is generated is able to significantly enhance the Raman detection of analytes applied to its surface.

The method that is devised to control the size of gold clusters and relative amount of gold within the formed nanospheroids is to cyclically ionize the substrate. The principle of this method is that, upon the initial cycle the laser pulses will ionize only a small layer of the surface. Each subsequent cycle will ionize further and further into the substrate. With appropriate tuning, control over the relative ratio of gold to silicon ion plume can be attained. In order to not completely ionize the gold nanolayer and create a primarily silicon nanospheroids, this mechanism is limited in terms of laser pulse power due to the threshold fluence. 


\subsection{2. $\mathrm{Ti} / \mathrm{TiO}_{2}$-Si hybrid nanostructures}

In previous studies that use an ultrafast femtosecond pulsed laser ionization mechanism to create Raman active nanostructures, $\mathrm{TiO}_{2}$ nanonetwork structures have been fabricated that produce significant SERS enhancement on the same scale as the Si nanostructures presented in this thesis. The proposed study for this future work would be to use the laser-ionization mechanism to create $\mathrm{TiO}_{2} / \mathrm{Si}$ hybrid nanostructures that have the potential to exhibit the combined SERS enhancement properties of both the $\mathrm{TiO}_{2}$ and the $\mathrm{Si}$ nanostructures. This study has the possibility to create a one-of-kind nanohybrid structure that consists of a $\mathrm{Ti}^{-\mathrm{TiO}_{2}}-\mathrm{Si}$ alloy that produces SERS activation through multiple charge-transfer sources, potentially leading to SERS enhancement not yet reported for semiconductor/meal oxide nanostructures.

\subsubsection{Multisource Au boosting of Si nanostructures: internal and external NMNs}

The studies presented in this thesis outline the contributions to SERS biosensing enhancement using a highly SERS active Si nanostructure combined with external noble metal nanostructures. The proposed study for this future work is to explore the SERS activity, sensitivity and reproducibility of Si nanostructures that contain internal Au nanoclusters and external NMNs for the detection of biomolecule analytes. This nanostructure would produce a charge-transfer SERS contribution from the Si nanostructure and a multisource plasmonic SERS from the internal and external NMNs that has the potential to exhibit SERS enhancement yet to be observed for biomolecule detection methods. In addition, the possibility of single-molecule sensitivity will be explored along with these nanostructures ability to sense more complex biomolecules.

\subsection{4. $\mathrm{Cu} / \mathrm{CuO} \mathrm{Si}$ nanohybrid structures}

This thesis has demonstrated that the laser-ionization mechanism can produce a miscible nanostructure containing $\mathrm{Au}$ and $\mathrm{Si}$ that produces ultrahigh SERS sensitivity for biomolecule detection. The proposed study for this future work is to determine the SERS characteristics of a $\mathrm{Cu} / \mathrm{CuO}-\mathrm{Si}$ alloy like nanostructure. Copper has similar plasmonic activity to noble metals and can generate SPR in the visible to NIR wavelength ranges; $\mathrm{CuO}$ has also been established in the literature as a SERS active material. This study could potentially produce a hybrid plasmonic/charge-transfer SERS sensitive nanostructure that has yet to be reported. 
6.2.5. DNA detection using Si-based nanostructure: Labelled and Label-free SERS detection of DNA

Throughout this thesis, Si nanostructures have been proven to be ultrasensitive SERS platforms for the detection of biomolecules. In this proposed future work, the use these Si nanostructures will be expanded to establish these nanostructures as SERS sensitive substrates for label and labelfree DNA detection. 


\section{$\underline{\text { References }}$}

1-1. Svitkova, V., et al., Assessment of Cds Quantum Dots Effect on Uv Damage to DNA Using a DNA/Quantum Dots Structured Electrochemical Biosensor and DNA Biosensing in Solution. Sensors and Actuators B: Chemical 2017, 243, 435-444.

1-2. Buck, R. P.; Lindner, E.; Kutner, W.; Inzelt, G., Piezoelectric Chemical Sensors - (Iupac Technical Report). Pure Appl Chem 2004, 76, 1139-1160.

1-3. Janata, J.; Bezegh, A., Chemical Sensors. Analytical chemistry 1988, 60, 62R-74R.

1-4. Xu, H.; Xue, C.; Zhang, R.; Chen, Y.; Li, F.; Shen, Z.; Jia, L.; Wu, Z.-S., Exponential Rolling Circle Amplification and Its Sensing Application for Highly Sensitive DNA Detection of Tumor Suppressor Gene. Sensors and Actuators B: Chemical 2017, 243, 1240-1247.

1-5. Fleischmann, M.; Hendra, P. J.; McQuillan, A. J., Raman Spectra of Pyridine Adsorbed at a Silver Electrode. Chemical Physics Letters 1974, 26.

1-6. Wei, H.; Xu, H., Hot Spots in Different Metal Nanostructures for Plasmon-Enhanced Raman Spectroscopy. Nanoscale 2013, 5, 10794-805.

1-7. Lombardi, J. R.; Birke, R. L.; Lu, T. H.; Xu, J., Charge-Transfer Theory of Surface Enhanced Raman-Spectroscopy - Herzberg-Teller Contributions. J Chem Phys 1986, 84, 41744180 .

1-8. Lombardi, J. R.; Birke, R. L., A Unified Approach to Surface-Enhanced Raman Spectroscopy. J Phys Chem C 2008, 112, 5605-5617.

1-9. Cong, S., et al., Noble Metal-Comparable Sers Enhancement from Semiconducting Metal Oxides by Making Oxygen Vacancies. Nat Commun 2015, 6, 7800.

1-10. Maznichenko, D.; Selvaganapathy, P. R.; Venkatakrishnan, K.; Tan, B., Tio2 Nanofibrous Interface Development for Raman Detection of Environmental Pollutants. Applied Physics Letters $2012,101,231602$. 
1-11. Maznichenko, D.; Venkatakrishnan, K.; Tan, B., Stimulating Multiple Sers Mechanisms by a Nanofibrous Three-Dimensional Network Structure of Titanium Dioxide (Tio2). The Journal of Physical Chemistry C 2013, 117, 578-583.

1-12. Cho, D.; Kim, M.; Hwang, J.; Park, J. H.; Joo, Y. L.; Jeong, Y., Facile Synthesis of Porous Silicon Nanofibers by Magnesium Reduction for Application in Lithium Ion Batteries. Nanoscale Res Lett 2015, 10, 424.

1-13. Le Ru, E. C.; Blackie, E.; Meyer, M.; Etchegoin, P. G., Surface Enhanced Raman Scattering Enhancement Factors: A Comprehensive Study. The Journal of Physical Chemistry C 2007, 111, 13794-13803.

1-14. Zheng, M.; Zhu, X.; Chen, Y.; Xiang, Q.; Duan, H., Three-Dimensional Donut-Like Gold Nanorings with Multiple Hot Spots for Surface-Enhanced Raman Spectroscopy. Nanotechnology 2017, 28, 045303.

1-15. Focsan, M.; Campu, A.; Craciun, A. M.; Potara, M.; Leordean, C.; Maniu, D.; Astilean, S., A Simple and Efficient Design to Improve the Detection of Biotin-Streptavidin Interaction with Plasmonic Nanobiosensors. Biosensors \& bioelectronics 2016, 86, 728-35.

1-16. Simoncelli, S.; Roller, E. M.; Urban, P.; Schreiber, R.; Turberfield, A. J.; Liedl, T.; Lohmuller, T., Quantitative Single-Molecule Surface-Enhanced Raman Scattering by Optothermal Tuning of DNA Origami-Assembled Plasmonic Nanoantennas. ACS Nano 2016, 10, 9809-9815.

1-17. Nuntawong, N., et al., Detection of Methamphetamine/Amphetamine in Human Urine Based on Surface-Enhanced Raman Spectroscopy and Acidulation Treatments. Sensors and Actuators B: Chemical 2017, 239, 139-146.

1-18. Lin, K.-Q.; Yi, J.; Hu, S.; Liu, B.-J.; Liu, J.-Y.; Wang, X.; Ren, B., Size Effect on Sers of Gold Nanorods Demonstrated Via Single Nanoparticle Spectroscopy. The Journal of Physical Chemistry C 2016, 120, 20806-20813.

1-19. Xu, Q.; Guo, X.; Xu, L.; Ying, Y.; Wu, Y.; Wen, Y.; Yang, H., Template-Free Synthesis of Sers-Active Gold Nanopopcorn for Rapid Detection of Chlorpyrifos Residues. Sensors and Actuators B: Chemical 2017, 241, 1008-1013. 
1-20. Bryche, J.-F.; Bélier, B.; Bartenlian, B.; Barbillon, G., Low-Cost Sers Substrates Composed of Hybrid Nanoskittles for a Highly Sensitive Sensing of Chemical Molecules. Sensors and Actuators B: Chemical 2017, 239, 795-799.

1-21. Jiji, S. G.; Gopchandran, K. G., Au-Ag Hollow Nanostructures with Tunable Sers Properties. Spectrochimica acta. Part A, Molecular and biomolecular spectroscopy 2017, 171, 499506.

1-22. Liu, K.; Bai, Y.; Zhang, L.; Yang, Z.; Fan, Q.; Zheng, H.; Yin, Y.; Gao, C., Porous Au-Ag Nanospheres with High-Density and Highly Accessible Hotspots for Sers Analysis. Nano Lett 2016, 16, 3675-81.

1-23. Song, C.; Yang, B.; Zhu, Y.; Yang, Y.; Wang, L., Ultrasensitive Sliver Nanorods Array Sers Sensor for Mercury Ions. Biosensors \& bioelectronics 2017, 87, 59-65.

1-24. He, L.; Ai, C.; Wang, W.; Gao, N.; Yao, X.; Tian, C.; Zhang, K., An Effective ThreeDimensional Surface-Enhanced Raman Scattering Substrate Based on Oblique Si Nanowire Arrays Decorated with Ag Nanoparticles. Journal of Materials Science 2016, 51, 3854-3860.

1-25. Wei, Y.; Zhu, Y.-y.; Wang, M.-1., Surface-Enhanced Raman Spectroscopy of Gastric Cancer Serum with Gold Nanoparticles/Silicon Nanowire Arrays. Optik - International Journal for Light and Electron Optics 2016, 127, 7902-7907.

1-26. Hou, T.; Liu, Y.; Xu, L.; Wu, Y.; Ying, Y.; Wen, Y.; Guo, X.; Yang, H., Au Dotted Magnetic Graphene Sheets for Sensitive Detection of Thiocyanate. Sensors and Actuators B: Chemical 2017, 241, 376-382.

1-27. Zheng, H.; Ni, D.; Yu, Z.; Liang, P., Preparation of Sers-Active Substrates Based on Graphene Oxide/Silver Nanocomposites for Rapid Zdetection of L-Theanine. Food chemistry 2017, 217, 511-6.

1-28. Li, T.; Wu, K.; Rindzevicius, T.; Wang, Z.; Schulte, L.; Schmidt, M. S.; Boisen, A.; Ndoni, S., Wafer-Scale Nanopillars Derived from Block Copolymer Lithography for Surface-Enhanced Raman Spectroscopy. ACS applied materials \& interfaces 2016, 8, 15668-75.

1-29. Son, J. G.; Han, S. W.; Wi, J. S.; Lee, T. G., Guided Formation of Sub-5 Nm Interstitial Gaps between Plasmonic Nanodisks. Nanoscale 2015, 7, 8338-42. 
1-30. Dai, H.; Sun, Y.; Ni, P.; Lu, W.; Jiang, S.; Wang, Y.; Li, Z.; Li, Z., Three-Dimensional Tio2 Supported Silver Nanoparticles as Sensitive and Uv-Cleanable Substrate for Surface Enhanced Raman Scattering. Sensors and Actuators B: Chemical 2017, 242, 260-268.

1-31. Li, Y.; Zhou, L.; Tang, L.; Li, M.; He, J.-J., Improved Surface Enhanced Raman Scattering Based on Hybrid Au Nanostructures for Biomolecule Detection. IEEE Photonics Journal 2016, 8, $1-7$.

1-32. Qiu, S., et al., Early Discrimination of Nasopharyngeal Carcinoma Based on Tissue Deoxyribose Nucleic Acid Surface-Enhanced Raman Spectroscopy Analysis. Journal of biomedical optics 2016, 21, 125003.

1-33. Ma, X. Y.; Liu, Y.; Zhou, N. X.; Duan, N.; Wu, S. J.; Wang, Z. P., Sers Aptasensor Detection of Salmonella Typhimurium Using a Magnetic Gold Nanoparticle and Gold Nanoparticle Based Sandwich Structure. Analytical Methods 2016, 8, 8099-8105.

1-34. Tan, X.; Melkersson, J.; Wu, S.; Wang, L.; Zhang, J., Noble-Metal-Free Materials for Surface-Enhanced Raman Spectroscopy Detection. Chemphyschem : a European journal of chemical physics and physical chemistry 2016, 17, 2630-9.

1-35. Yamada, H.; Yamamoto, Y., Surface Enhanced Raman-Scattering (Sers) of Chemisorbed Species on Various Kinds of Metals and Semiconductors. Surface Science 1983, 134, 71-90.

1-36. Hossain, M. M.; Shima, H.; Islam, M. A.; Hasan, M.; Lee, M., Synergetic Effect in Raman Scattering of Zno Nanoparticles in Zno-Cnt Fibers: A Way to Enhance the G and 2d Band. The Journal of Physical Chemistry C 2016, 120, 17670-17682.

1-37. Bontempi, N.; Carletti, L.; De Angelis, C.; Alessandri, I., Plasmon-Free Sers Detection of Environmental Co2 on Tio2 Surfaces. Nanoscale 2016, 8, 3226-31.

1-38. Wang, Y.; Sun, Z.; Hu, H.; Jing, S.; Zhao, B.; Xu, W.; Zhao, C.; Lombardi, J. R., Raman Scattering Study of Molecules Adsorbed on Zns Nanocrystals. Journal of Raman Spectroscopy 2007, 38, 34-38.

1-39. Wang, R.-C.; Lin, H.-Y., Efficient Surface Enhanced Raman Scattering from Cu2o Porous Nanowires Transformed from Cuo Nanowires by Plasma Treatments. Materials Chemistry and Physics 2012, 136, 661-665. 
1-40. Mou, C. B.; He, T. J.; Wang, X. Y.; Liu, F. C.; Jiang, J. S.; Chen, L. W., Surface Enhanced Raman Scattering (Sers) from H2tspp and Ag(Ii)Tspp Adsorbed on Uniform Fe3o4 Colloids. Acta Phys-Chim Sin 1996, 12, 841-844.

1-41. Wang, Y. F.; Zhang, J. H.; Jia, H. Y.; Li, M. J.; Zeng, J. B.; Yang, B.; Zhao, B.; Xu, W. Q.; Lombardi, J. R., Mercaptopyridine Surface-Functionalized Cdte Quantum Dots with Enhanced Raman Scattering Properties. J Phys Chem C 2008, 112, 996-1000.

1-42. Fu, X.; Pan, Y.; Wang, X.; Lombardi, J. R., Quantum Confinement Effects on ChargeTransfer between Pbs Quantum Dots and 4-Mercaptopyridine. J Chem Phys 2011, 134, 024707.

1-43. Yang, L.; Gong, M.; Jiang, X.; Chen, Y.; Han, X.; Song, K.; Sun, X.; Zhang, Y.; Zhao, B., Sers Investigation and Detection of Levofloxacin Drug Molecules on Semiconductor Tio2: Charge Transfer Contribution. Colloids and Surfaces A: Physicochemical and Engineering Aspects 2016, 508, 142-149.

1-44. Tan, X. J.; Wang, L. Z.; Cheng, C.; Yan, X. F.; Shen, B.; Zhang, J. L., Plasmonic Moo3X@Moo3 Nanosheets for Highly Sensitive Sers Detection through Nanoshell-Isolated Electromagnetic Enhancement. Chemical Communications 2016, 52, 2893-2896.

1-45. Han, X. X.; Kohler, C.; Kozuch, J.; Kuhlmann, U.; Paasche, L.; Sivanesan, A.; Weidinger, I. M.; Hildebrandt, P., Potential-Dependent Surface-Enhanced Resonance Raman Spectroscopy at Nanostructured Tio2 : A Case Study on Cytochrome B5. Small 2013, 9, 4175-81.

1-46. Britto Hurtado, R., et al., Instant Synthesis of Gold Nanoparticles at Room Temperature and Sers Applications. Physics Letters A 2016, 380, 2658-2663.

1-47. Li, W. B.; Guo, Y. Y.; Zhang, P., Sers-Active Silver Nanoparticles Prepared by a Simple and Green Method. J Phys Chem C 2010, 114, 6413-6417.

1-48. D'Andrea, C., et al., Decoration of Silicon Nanowires with Silver Nanoparticles for Ultrasensitive Surface Enhanced Raman Scattering. Nanotechnology 2016, 27, 375603.

1-49. Alubaidy, M.; Soleymani, L.; Venkatakrishnan, K.; Tan, B., Femtosecond Laser Nanostructuring for Femtosensitive DNA Detection. Biosensors \& bioelectronics 2012, 33, 82-7. 
1-50. Premnath, P.; Tan, B.; Venkatakrishnan, K., Engineering Functionalized Multi-Phased Silicon/Silicon Oxide Nano-Biomaterials to Passivate the Aggressive Proliferation of Cancer. Scientific reports 2015, 5, 12141.

2-1 Shiohara, A.; Wang, Y. S.; Liz-Marzan, L. M., Recent Approaches toward Creation of Hot Spots for Sers Detection. Journal of Photochemistry and Photobiology C-Photochemistry Reviews 2014, 21, 2-25.

2-2. Sharma, B.; Frontiera, R. R.; Henry, A. I.; Ringe, E.; Van Duyne, R. P., Sers: Materials, Applications, and the Future. Materials Today 2012, 15, 16-25.

2-3. Liu, H.; Zhang, L.; Lang, X.; Yamaguchi, Y.; Iwasaki, H.; Inouye, Y.; Xue, Q.; Chen, M., Single Molecule Detection from a Large-Scale Sers-Active Au(7)(9)Ag(2)(1) Substrate. Scientific reports $2011,1,112$.

2-4. Mulvihill, M.; Tao, A.; Benjauthrit, K.; Arnold, J.; Yang, P., Surface-Enhanced Raman Spectroscopy for Trace Arsenic Detection in Contaminated Water. Angew Chem Int Ed Engl 2008, 47, 6456-60.

2-5. Dasary, S. S.; Singh, A. K.; Senapati, D.; Yu, H.; Ray, P. C., Gold Nanoparticle Based Label-Free Sers Probe for Ultrasensitive and Selective Detection of Trinitrotoluene. Journal of the American Chemical Society 2009, 131, 13806-12.

2-6. Li, Y. S.; Church, J. S., Raman Spectroscopy in the Analysis of Food and Pharmaceutical Nanomaterials. J Food Drug Anal 2014, 22, 29-48.

2-7. Wang, X.; Qian, X.; Beitler, J. J.; Chen, Z. G.; Khuri, F. R.; Lewis, M. M.; Shin, H. J.; Nie, S.; Shin, D. M., Detection of Circulating Tumor Cells in Human Peripheral Blood Using SurfaceEnhanced Raman Scattering Nanoparticles. Cancer Res 2011, 71, 1526-32.

2-8. Zhu, J.; Zhou, J.; Guo, J.; Cai, W.; Liu, B.; Wang, Z.; Sun, Z., Surface-Enhanced Raman Spectroscopy Investigation on Human Breast Cancer Cells. Chem Cent J 2013, 7, 37.

2-9. Ondera, T. J.; Hamme, A. T., 2nd, Gold Nanopopcorn Attached Single-Walled Carbon Nanotube Hybrid for Rapid Detection and Killing of Bacteria. J Mater Chem B Mater Biol Med 2014, 2, 7534-7543. 
2-10. Cao, Y. C.; Jin, R.; Mirkin, C. A., Nanoparticles with Raman Spectroscopic Fingerprints for DNA and Rna Detection. Science 2002, 297, 1536-40.

2-11. Li, J.; Song, S.; Li, D.; Su, Y.; Huang, Q.; Zhao, Y.; Fan, C., Multi-Functional Crosslinked Au Nanoaggregates for the Amplified Optical DNA Detection. Biosensors \& bioelectronics 2009, $24,3311-5$.

2-12. Luo, S. C.; Sivashanmugan, K.; Liao, J. D.; Yao, C. K.; Peng, H. C., Nanofabricated SersActive Substrates for Single-Molecule to Virus Detection in Vitro: A Review. Biosensors \& bioelectronics 2014, 61, 232-40.

2-13. Smith, E.; Dent, G., Modern Raman Spectroscopy: A Practical Approach; John Wiley \& Sons Ltd, 2005.

2-14. Haynes, C. L.; McFarland, A. D.; Duyne, R. P. V., Surface-Enhanced Raman Spectroscopy. Analytical chemistry 2005, 77, 338 A-346 A.

2-15. Jain, P. K.; Huang, X.; El-Sayed, I. H.; El-Sayad, M. A., Review of Some Interesting Surface Plasmon Resonance-Enhanced Properties of Noble Metal Nanoparticles and Their Applications to Biosystems. Plasmonics 2007, 2, 107-118.

2-16. Khlebtsov, B.; Panfilova, E.; Khanadeev, V.; Khlebtsov, N., Improved Size-Tunable Synthesis and Sers Properties of Au Nanostars. Journal of Nanoparticle Research 2014, 16.

2-17. Wang, X. T.; Shi, W. S.; She, G. W.; Mu, L. X.; Lee, S. T., High-Performance SurfaceEnhanced Raman Scattering Sensors Based on Ag Nanoparticles-Coated Si Nanowire Arrays for Quantitative Detection of Pesticides. Applied Physics Letters 2010, 96, 053104.

2-18. Mahmoud, M. A.; O'Neil, D.; El-Sayed, M. A., Hollow and Solid Metallic Nanoparticles in Sensing and in Nanocatalysis. Chemistry of Materials 2014, 26, 44-58.

2-19. Gellner, M.; Kustner, B.; Schlucker, S., Optical Properties and Sers Efficiency of Tunable Gold/Silver Nanoshells. Vibrational Spectroscopy 2009, 50, 43-47.

2-20. Tong, L. M.; Xu, H. X.; Kall, M., Nanogaps for Sers Applications. Mrs Bulletin 2014, 39, 163-168. 
2-21. Hatab, N. A.; Hsueh, C. H.; Gaddis, A. L.; Retterer, S. T.; Li, J. H.; Eres, G.; Zhang, Z.; Gu, B., Free-Standing Optical Gold Bowtie Nanoantenna with Variable Gap Size for Enhanced Raman Spectroscopy. Nano Lett 2010, 10, 4952-5.

2-22. Wells, S. M.; Merkulov, I. A.; Kravchenko, I. I.; Lavrik, N. V.; Sepaniak, M. J., Silicon Nanopillars for Field-Enhanced Surface Spectroscopy. Acs Nano 2012, 6, 2948-2959.

2-23. Wang, X.; Shi, W.; She, G.; Mu, L., Using Si and Ge Nanostructures as Substrates for Surface-Enhanced Raman Scattering Based on Photoinduced Charge Transfer Mechanism. Journal of the American Chemical Society 2011, 133, 16518-23.

2-24. Khorasaninejad, M.; Walia, J.; Saini, S. S., Enhanced First-Order Raman Scattering from Arrays of Vertical Silicon Nanowires. Nanotechnology 2012, 23, 275706.

2-25. Cao, L.; Nabet, B.; Spanier, J. E., Enhanced Raman Scattering from Individual Semiconductor Nanocones and Nanowires. Phys Rev Lett 2006, 96, 157402.

2-26. Garcia-Leis, A.; Garcia-Ramos, J. V.; Sanchez-Cortes, S., Silver Nanostars with High Sers Performance. The Journal of Physical Chemistry C 2013, 117, 7791-7795.

2-27. Wang, Y.; Chen, H.; Wang, E., Facile Fabrication of Gold Nanoparticle Arrays for Efficient Surface-Enhanced Raman Scattering. Nanotechnology 2008, 19, 105604.

2-28. Tao, A.; Kim, F.; Hess, C.; Goldberger, J.; He, R. R.; Sun, Y. G.; Xia, Y. N.; Yang, P. D., Langmuir-Blodgett Silver Nanowire Monolayers for Molecular Sensing Using Surface-Enhanced Raman Spectroscopy. Nano Letters 2003, 3, 1229-1233.

2-29. Jiang, Z. Y.; Jiang, X. X.; Su, S.; Wei, X. P.; Lee, S. T.; He, Y., Silicon-Based Reproducible and Active Surface-Enhanced Raman Scattering Substrates for Sensitive, Specific, and Multiplex DNA Detection. Applied Physics Letters 2012, 100, 203104.

2-30. He, Y.; Su, S.; Xu, T.; Zhong, Y.; Zapien, J. A.; Li, J.; Fan, C.; Lee, S.-T., Silicon Nanowires-Based Highly-Efficient Sers-Active Platform for Ultrasensitive DNA Detection. Nano Today 2011, 6, 122-130.

2-31. Kahl, M.; Voges, E.; Kostrewa, S.; Viets, C.; Hill, W., Periodically Structured Metallic Substrates for Sers. Sensors and Actuators B: Chemical 1998, 51, 285-291. 
2-32. Sun, Z.; Zhao, B.; Lombardi, J. R., Zno Nanoparticle Size-Dependent Excitation of Surface Raman Signal from Adsorbed Molecules: Observation of a Charge-Transfer Resonance. Applied Physics Letters 2007, 91, 221106.

2-33. Wang, Y.; Sun, Z.; Hu, H.; Jing, S.; Zhao, B.; Xu, W.; Zhao, C.; Lombardi, J. R., Raman Scattering Study of Molecules Adsorbed on Zns Nanocrystals. Journal of Raman Spectroscopy 2007, 38, 34-38.

2-34. Wang, Y.; Sun, Z.; Wang, Y.; Hu, H.; Zhao, B.; Xu, W.; Lombardi, J. R., SurfaceEnhanced Raman Scattering on Mercaptopyridine-Capped Cds Microclusters. Spectrochimica acta. Part A, Molecular and biomolecular spectroscopy 2007, 66, 1199-203.

2-35. Wang, Y. F.; Hu, H. L.; Jing, S. Y.; Wang, Y. X.; Sun, Z. H.; Zhao, B.; Zhao, C.; Lombardi, J. R., Enhanced Raman Scattering as a Probe for 4-Mercaptopyridine Surface-Modified Copper Oxide Nanocrystals. Analytical Sciences 2007, 23, 787-791.

2-36. Maznichenko, D.; Selvaganapathy, P. R.; Venkatakrishnan, K.; Tan, B., TiO2 Nanofibrous Interface Development for Raman Detection of Environmental Pollutants. Applied Physics Letters 2012, 101, 231602.

2-37. Han, X. X.; Kohler, C.; Kozuch, J.; Kuhlmann, U.; Paasche, L.; Sivanesan, A.; Weidinger, I. M.; Hildebrandt, P., Potential-Dependent Surface-Enhanced Resonance Raman Spectroscopy at Nanostructured Tio2 : A Case Study on Cytochrome B5. Small 2013, 9, 4175-81.

2-38. Musumeci, A.; Gosztola, D.; Schiller, T.; Dimitrijevic, N. M.; Mujica, V.; Martin, D.; Rajh, T., Sers of Semiconducting Nanoparticles (Tio2 Hybrid Composites). Journal of the American Chemical Society 2009, 131, 6040-+.

2-39. Alessandri, I., Enhancing Raman Scattering without Plasmons: Unprecedented Sensitivity Achieved by Tio2 Shell-Based Resonators. Journal of the American Chemical Society 2013, 135, $5541-4$.

2-40. Lombardi, J. R.; Birke, R. L., Theory of Surface-Enhanced Raman Scattering in Semiconductors. The Journal of Physical Chemistry C 2014, 118, 11120-11130.

2-41. Tan, B.; Venkatakrishnan, K., Synthesis of Fibrous Nanoparticle Aggregates by Femtosecond Laser Ablation in Air. Opt Express 2009, 17, 1064-9. 
2-42. Gamaly, E. G.; Rode, A. V.; Luther-Davies, B., Ultrafast Ablation with High-Pulse-Rate Lasers. Part I: Theoretical Considerations. Journal of Applied Physics 1999, 85, 4213-4221.

2-43. Kluge, M. D.; Ray, J. R.; Rahman, A., Amorphous-Silicon Formation by Rapid Quenching: A Molecular-Dynamics Study. Phys Rev B Condens Matter 1987, 36, 4234-4237.

2-44. Kiani, A.; Venkatakrishnan, K.; Tan, B., Micro/Nano Scale Amorphization of Silicon by Femtosecond Laser Irradiation. Opt Express 2009, 17, 16518-26.

2-45. Xia, L., et al., Surface Enhanced Raman Scattering Substrate with Metallic Nanogap Array Fabricated by Etching the Assembled Polystyrene Spheres Array. Opt Express 2013, 21, 1134955.

2-46. Maznichenko, D.; Venkatakrishnan, K.; Tan, B., Stimulating Multiple Sers Mechanisms by a Nanofibrous Three-Dimensional Network Structure of Titanium Dioxide (Tio2). The Journal of Physical Chemistry C 2013, 117, 578-583.

2-47. Fleischmann, M.; Hendra, P. J.; McQuillan, A. J., Raman Spectra of Pyridzne Adsorbed at a Silver Electrode. Chemical Physics Letters 1974, 26.

2-48. Zaidi, S. H.; Chu, A. S.; Brueck, S. R. J., Optical Properties of Nanoscale, OneDimensional Silicon Grating Structures. Journal of Applied Physics 1996, 80, 6997-7008.

2-49. Callister, W. D., Materials Science and Engineering: An Introduction, 7th ed.; Wiley, 2006.

2-50. Veprek, S.; Sarott, F.; Iqbal, Z., Effect of Grain Boundaries on the Raman Spectra, Optical Absorption, and Elastic Light Scattering in Nanometer-Sized Crystalline Silicon. Physical Review B 1987, 36, 3344-3350.

2-51. Wu, H. Y.; Choi, C. J.; Cunningham, B. T., Plasmonic Nanogap-Enhanced Raman Scattering Using a Resonant Nanodome Array. Small 2012, 8, 2878-85.

2-52. Suh, Y. D.; Schenter, G. K.; Zhu, L.; Lu, H. P., Probing Nanoscale Surface Enhanced Raman-Scattering Fluctuation Dynamics Using Correlated Afm and Confocal Ultramicroscopy. Ultramicroscopy 2003, 97, 89-102. 
2-53. Kleinman, S. L.; Frontiera, R. R.; Henry, A. I.; Dieringer, J. A.; Van Duyne, R. P., Creating, Characterizing, and Controlling Chemistry with Sers Hot Spots. Phys Chem Chem Phys 2013, 15, 21-36.

2-54. Verma, S.; Rao, B. T.; Srivastava, A. K.; Patel, H. S.; Satapathy, S.; Joshi, M. P.; Sahu, V. K.; Kukreja, L. M., Studies on Interdependent Optical Properties of Rhodamine 6g Dye and Gold Nanoparticles at Different Dilutions of Aqueous Solutions. Journal of Luminescence 2014, 155, 156-164.

2-55. Chang, C. C.; Yang, K. H.; Liu, Y. C.; Yu, C. C.; Wu, Y. H., Surface-Enhanced Raman Scattering-Active Gold Nanoparticles Modified with a Monolayer of Silver Film. The Analyst 2012, 137, 4943-50.

2-56. Zheng, J.; Hu, Y.; Bai, J.; Ma, C.; Li, J.; Li, Y.; Shi, M.; Tan, W.; Yang, R., Universal Surface-Enhanced Raman Scattering Amplification Detector for Ultrasensitive Detection of Multiple Target Analytes. Analytical chemistry 2014, 86, 2205-12.

2-57. Yuan, H.; Khoury, C. G.; Hwang, H.; Wilson, C. M.; Grant, G. A.; Vo-Dinh, T., Gold Nanostars: Surfactant-Free Synthesis, 3d Modelling, and Two-Photon Photoluminescence Imaging. Nanotechnology 2012, 23, 075102.

2-58. Fan, M.; Andrade, G. F.; Brolo, A. G., A Review on the Fabrication of Substrates for Surface Enhanced Raman Spectroscopy and Their Applications in Analytical Chemistry. Anal Chim Acta 2011, 693, 7-25.

2-59. Braun, G.; Pavel, I.; Morrill, A. R.; Seferos, D. S.; Bazan, G. C.; Reich, N. O.; Moskovits, M., Chemically Patterned Microspheres for Controlled Nanoparticle Assembly in the Construction of Sers Hot Spots. Journal of the American Chemical Society 2007, 129, 7760-1.

2-60. Rigo, M. V.; Seo, J.; Kim, W. J.; Jung, S., Plasmon Coupling of R6g-Linked Gold Nanoparticle Assemblies for Surface-Enhanced Raman Spectroscopy. Vibrational Spectroscopy 2011, 57, 315-318.

2-61. Le Ru, E. C.; Blackie, E.; Meyer, M.; Etchegoin, P. G., Surface Enhanced Raman Scattering Enhancement Factors: A Comprehensive Study. The Journal of Physical Chemistry C 2007, 111, 13794-13803. 
3-1. Cui, L.; Wu, J.; Ju, H., Electrochemical Sensing of Heavy Metal Ions with Inorganic, Organic and Bio-Materials. Biosensors \& bioelectronics 2015, 63, 276-86.

3-2. Minami, T.; Sasaki, Y.; Minamiki, T.; Wakida, S.-i.; Kurita, R.; Niwa, O.; Tokito, S., Selective Nitrate Detection by an Enzymatic Sensor Based on an Extended-Gate Type Organic Field-Effect Transistor. Biosensors and Bioelectronics 2016, 81, 87-91.

3-3. Yang, Z.-p.; Liu, X.; Zhang, C.-j.; Liu, B.-Z., A High-Performance Nonenzymatic Piezoelectric Sensor Based on Molecularly Imprinted Transparent Tio2 Film for Detection of Urea. Biosensors and Bioelectronics 2015, 74, 85-90.

3-4. Wandermur, G.; Rodrigues, D.; Allil, R.; Queiroz, V.; Peixoto, R.; Werneck, M.; Miguel, M., Plastic Optical Fiber-Based Biosensor Platform for Rapid Cell Detection. Biosensors \& bioelectronics 2014, 54, 661-6.

3-5. Ramon-Marquez, T.; Medina-Castillo, A. L.; Fernandez-Gutierrez, A.; FernandezSanchez, J. F., Novel Optical Sensing Film Based on a Functional Nonwoven Nanofibre Mat for an Easy, Fast and Highly Selective and Sensitive Detection of Tryptamine in Beer. Biosensors \& bioelectronics 2016, 79, 600-7.

3-6. Laariedh, F.; Sow, I.; Ferchichi, A.; Zelsmann, M.; Boussey, J., Large-Area, Cost-Effective Surface-Enhanced Raman Scattering (Sers) Substrates Fabrication. Microelectronic Engineering 2015, 145, 124-127.

3-7. Kang, H.; Heo, C. J.; Jeon, H. C.; Lee, S. Y.; Yang, S. M., Durable Plasmonic Cap Arrays on Flexible Substrate with Real-Time Optical Tunability for High-Fidelity Sers Devices. ACS applied materials \& interfaces 2013, 5, 4569-74.

3-8. Xiao, R.; Wang, C. W.; Zhu, A. N.; Long, F., Single Functional Magnetic-Bead as Universal Biosensing Platform for Trace Analyte Detection Using Sers-Nanobioprobe. Biosensors \& bioelectronics 2016, 79, 661-8.

3-9. Giordano, M. C.; Foti, A.; Messina, E.; Gucciardi, P. G.; Comoretto, D.; Buatier de Mongeot, F., Sers Amplification from Self-Organized Arrays of Plasmonic Nanocrescents. ACS applied materials \& interfaces 2016, 8, 6629-38. 
3-10. Prinz, J.; Heck, C.; Ellerik, L.; Merk, V.; Bald, I., DNA Origami Based Au-Ag-Core-Shell Nanoparticle ingle-Molecule Sers Sensitivity. Nanoscale 2016, 8, 5612-20.

3-11. Yang, K.; Hu, Y.; Dong, N., A Novel Biosensor Based on Competitive Sers Immunoassay and aration for Accurate and Sensitive Detection of Chloramphenicol. Biosensors \& bioelectronics 2016, 80, 373-7.

3-12. Rey, D. A.; Strickland, A. D.; Kirui, D.; Niamsiri, N.; Batt, C. A., In Vitro Self-Assembly of Gold Nanoparticle-Coated Poly(3-Hydroxybutyrate) Granules Exhibiting Plasmon-Induced Thermo-Optical Enhancements. ACS applied materials \& interfaces 2010, 2, 1804-10.

3-13. Sun, K.; Huang, Q.; Meng, G.; Lu, Y., Highly Sensitive and Selective Surface-Enhanced Raman Spectroscopy Label-Free Detection of 3,3',4,4'-Tetrachlorobiphenyl Using DNA AptamerModified Ag-Nanorod Arrays. ACS applied materials \& interfaces 2016, 8, 5723-5728.

3-14. Bryche, J.-F.; Tsigara, A.; Bélier, B.; de la Chapelle, M. L.; Canva, M.; Bartenlian, B.; Barbillon, G., Surface Enhanced Raman Scattering Improvement of Gold Triangular Nanoprisms by a Gold Reflective Underlayer for Chemical Sensing. Sensors and Actuators B: Chemical 2016, $228,31-35$.

3-15. Podagatlapalli, G. K.; Hamad, S.; Rao, S. V., Trace-Level Detection of Secondary Explosives Using Hybrid Silver-Gold Nanoparticles and Nanostructures Achieved with Femtosecond Laser Ablation. The Journal of Physical Chemistry C 2015, 119, 16972-16983.

3-16. Wu, H. Y.; Cunningham, B. T., Point-of-Care Detection and Real-Time Monitoring of Intravenously Delivered Drugs Via Tubing with an Integrated Sers Sensor. Nanoscale 2014, 6, 5162-71.

3-17. Xu, L.; Yin, H.; Ma, W.; Kuang, H.; Wang, L.; Xu, C., Ultrasensitive Sers Detection of Mercury Based on the Assembled Gold Nanochains. Biosensors \& bioelectronics 2015, 67, 4726.

3-18. Ding, X.; Kong, L.; Wang, J.; Fang, F.; Li, D.; Liu, J., Highly Sensitive Sers Detection of Hg2+ Ions in Aqueous Media Using Gold Nanoparticles/Graphene Heterojunctions. ACS applied materials \& interfaces 2013, 5, 7072-8. 
3-19. Ilkhani, H.; Hughes, T.; Li, J.; Zhong, C. J.; Hepel, M., Nanostructured SersElectrochemical Biosensors for Testing of Anticancer Drug Interactions with DNA. Biosensors \& bioelectronics 2016, 80, 257-64.

3-20. Zhang, H.; Ma, X.; Liu, Y.; Duan, N.; Wu, S.; Wang, Z.; Xu, B., Gold Nanoparticles Enhanced Sers Aptasensor for the Simultaneous Detection of Salmonella Typhimurium and Staphylococcus Aureus. Biosensors \& bioelectronics 2015, 74, 872-7.

3-21. Lv, Y.; Qin, Y.; Svec, F.; Tan, T., Molecularly Imprinted Plasmonic Nanosensor for Selective Sers Detection of Protein Biomarkers. Biosensors \& bioelectronics 2016, 80, 433-41.

3-22. Wu, X.; Luo, L.; Yang, S.; Ma, X.; Li, Y.; Dong, C.; Tian, Y.; Zhang, L.; Shen, Z.; Wu, A., Improved Sers Nanoparticles for Direct Detection of Circulating Tumor Cells in the Blood. ACS applied materials \& interfaces 2015, 7, 9965-71.

3-23. Xu, L.; Yan, W.; Ma, W.; Kuang, H.; Wu, X.; Liu, L.; Zhao, Y.; Wang, L.; Xu, C., Sers Encoded Silver Pyramids for Attomolar Detection of Multiplexed Disease Biomarkers. Advanced materials 2015, 27, 1706-11.

3-24. Fu, X.; Cheng, Z.; Yu, J.; Choo, P.; Chen, L.; Choo, J., A Sers-Based Lateral Flow Assay Biosensor for Highly Sensitive Detection of Hiv-1 DNA. Biosensors \& bioelectronics 2016, 78, $530-7$.

3-25. Kögler, M.; Zhang, B.; Cui, L.; Shi, Y.; Yliperttula, M.; Laaksonen, T.; Viitala, T.; Zhang, K., Real-Time Raman Based Approach for Identification of Biofouling. Sensors and Actuators B: Chemical 2016, 230, 411-421.

3-26. Song, D.; Yang, R.; Wang, C.; Xiao, R.; Long, F., Reusable Nanosilver-Coated Magnetic Particles for Ultrasensitive Sers-Based Detection of Malachite Green in Water Samples. Scientific reports $2016,6,22870$.

3-27. Ramya, A. N.; Joseph, M. M.; Nair, J. B.; Karunakaran, V.; Narayanan, N.; Maiti, K. K., New Insight of Tetraphenylethylene-Based Raman Signatures for Targeted Sers Nanoprobe Construction toward Prostate Cancer Cell Detection. ACS applied materials \& interfaces 2016, 8, 10220-5. 
3-28. Chen, H. Y.; Lin, M. H.; Wang, C. Y.; Chang, Y. M.; Gwo, S., Large-Scale Hot Spot Engineering for Quantitative Sers at the Single-Molecule Scale. Journal of the American Chemical Society 2015, 137, 13698-705.

3-29. Tang, J.; Guo, H.; Chen, M.; Yang, J.; Tsoukalas, D.; Zhang, B.; Liu, J.; Xue, C.; Zhang, W., Wrinkled Ag Nanostructured Gratings Towards Single Molecule Detection by Ultrahigh Surface Raman Scattering Enhancement. Sensors and Actuators B: Chemical 2015, 218, 145-151.

3-30. Liu, H.; Zhang, L.; Lang, X.; Yamaguchi, Y.; Iwasaki, H.; Inouye, Y.; Xue, Q.; Chen, M., Single Molecule Detection from a Large-Scale Sers-Active Au(7)(9)Ag(2)(1) Substrate. Scientific reports $2011,1,112$.

3-31. Zhang, H.; Wang, C.; Wang, K.; Xuan, X.; Lv, Q.; Jiang, K., Ultrasensitive Fluorescent Ratio Imaging Probe for the Detection of Glutathione Ultratrace Change in Mitochondria of Cancer Cells. Biosensors \& bioelectronics 2016, 85, 96-102.

3-32. Khlebtsov, B. N.; Khanadeev, V. A.; Panfilova, E. V.; Bratashov, D. N.; Khlebtsov, N. G., Gold Nanoisland Films as Reproducible Sers Substrates for Highly Sensitive Detection of Fungicides. ACS applied materials \& interfaces 2015, 7, 6518-29.

3-33. Kim, J. E.; Choi, J. H.; Colas, M.; Kim, D. H.; Lee, H., Gold-Based Hybrid Nanomaterials for Biosensing and Molecular Diagnostic Applications. Biosensors \& bioelectronics 2016, 80, 543-59.

3-34. Zhao, Y.; Yang, Y.; Luo, Y.; Yang, X.; Li, M.; Song, Q., Double Detection of Mycotoxins Based on Sers Labels Embedded Ag@Au Core-Shell Nanoparticles. ACS applied materials \& interfaces 2015, 7, 21780-6.

3-35. Su, S.; Zhang, C.; Yuwen, L.; Chao, J.; Zuo, X.; Liu, X.; Song, C.; Fan, C.; Wang, L., Creating Sers Hot Spots on Mos(2) Nanosheets with in Situ Grown Gold Nanoparticles. ACS applied materials \& interfaces 2014, 6, 18735-41.

3-36. Xu, L.; Kuang, H.; Xu, C.; Ma, W.; Wang, L.; Kotov, N. A., Regiospecific Plasmonic Assemblies for in Situ Raman Spectroscopy in Live Cells. Journal of the American Chemical Society 2012, 134, 1699-709. 
3-37. Vo-Dinh, T.; Lieberman, R. A.; Gauglitz, G. G.; Liu, Y.; Yuan, H.; Fales, A. M.; Vo-Dinh, T., Plasmonics-Active Gold Nanostars for Chemical and Biological Sensing Using Sers Detection. 2015, 9486, 948607.

3-38. Wang, Y.; Polavarapu, L.; Liz-Marzan, L. M., Reduced Graphene Oxide-Supported Gold Nanostars for Improved Sers Sensing and Drug Delivery. ACS applied materials \& interfaces 2014, 6, 21798-805.

3-39. Wang, Y.; Wen, G.; Ye, L.; Liang, A.; Jiang, Z., Label-Free Sers Study of Galvanic Replacement Reaction on Silver Nanorod Surface and Its Application to Detect Trace Mercury Ion. Scientific reports 2016, 6, 19650.

3-40. Shao, J.; Tong, L.; Tang, S.; Guo, Z.; Zhang, H.; Li, P.; Wang, H.; Du, C.; Yu, X. F., Plla Nanofibrous Paper-Based Plasmonic Substrate with Tailored Hydrophilicity for Focusing Sers Detection. ACS applied materials \& interfaces 2015, 7, 5391-9.

3-41. Sinha, G.; Depero, L. E.; Alessandri, I., Recyclable Sers Substrates Based on Au-Coated Zno Nanorods. ACS applied materials \& interfaces 2011, 3, 2557-63.

3-42. Zhu, Y.; Kuang, H.; Xu, L.; Ma, W.; Peng, C.; Hua, Y.; Wang, L.; Xu, C., Gold Nanorodassembly Based Approach to Toxin Detection by Sers. J. Mater. Chem. 2012, 22, 23872391.

3-43. Khlebtsov, B. N.; Liu, Z.; Ye, J.; Khlebtsov, N. G., Au@Ag Core/Shell Cuboids and Dumbbells: Optical Properties and Sers Response. Journal of Quantitative Spectroscopy and Radiative Transfer 2015, 167, 64-75.

3-44. Wang, P.; Xia, M.; Liang, O.; Sun, K.; Cipriano, A. F.; Schroeder, T.; Liu, H.; Xie, Y. H., Label-Free Sers Selective Detection of Dopamine and Serotonin Using Graphene-Au Nanopyramid Heterostructure. Analytical chemistry 2015, 87, 10255-61.

3-45. Wang, Y.; Wang, Y.; Wang, H.; Cong, M.; Xu, W.; XU, S., Surface-Enhanced Raman Scattering on a Hierarchical Structural Ag Nano-Crown Array in Different Detection Ways. Phys Chem Chem Phys 2015, 17.

3-46. Wang, X.; Shi, W.; She, G.; Mu, L., Surface-Enhanced Raman Scattering (Sers) on Transition Metal and Semiconductor Nanostructures. Phys Chem Chem Phys 2012, 14, 5891-901. 
3-47. Alvarez, S. D.; Derfus, A. M.; Schwartz, M. P.; Bhatia, S. N.; Sailor, M. J., The Compatibility of Hepatocytes with Chemically Modified Porous Silicon with Reference to in Vitro Biosensors. Biomaterials 2009, 30, 26-34.

3-48. Qi, D.; Lu, L.; Wang, L.; Zhang, J., Improved Sers Sensitivity on Plasmon-Free Tio(2) Photonic Microarray by Enhancing Light-Matter Coupling. Journal of the American Chemical Society 2014, 136, 9886-9.

3-49. Yamada, H.; Yamamoto, Y.; Tani, N., Surface-Enhanced Raman Scattering (Sers) of Adsorbed Molecules on Smooth Surfaces of Metals and a Metal Oxide. Chemical Physics Letters 1981, 86, 4.

3-50. Hayashi, S.; Koh, R.; Ichiyama, Y.; Yamamoto, K., Evidence for Surface-Enhanced Raman Scattering on Nonmetallic Surfaces: Copper Phthalocyanine Molecules on Gap Small Particles. Phys Rev Lett 1988, 60, 1085-1088.

3-51. Li, W., et al., Cute Nanocrystals: Shape and Size Control, Plasmonic Properties, and Use as Sers Probes and Photothermal Agents. Journal of the American Chemical Society 2013, 135, 7098-101.

3-52. Islam, S. K.; Tamargo, M.; Moug, R.; Lombardi, J. R., Surface-Enhanced Raman Scattering on a Chemically Etched Znse Surface. The Journal of Physical Chemistry C 2013, 117, 23372-23377.

3-53. Lombardi, J. R.; Birke, R. L., Theory of Surface-Enhanced Raman Scattering in Semiconductors. The Journal of Physical Chemistry C 2014, 118, 11120-11130.

3-54. Jiang, L.; You, T.; Yin, P.; Shang, Y.; Zhang, D.; Guo, L.; Yang, S., Surface-Enhanced Raman Scattering Spectra of Adsorbates on Cu2o Nanospheres: Charge-Transfer and Electromagnetic Enhancement. Nanoscale 2013, 5, 2784.

3-55. Chang, L.; Xu, D.; Xue, X., Photoluminescence and Raman Scattering Study in Zno:Mg Nanocrystals. Journal of Materials Science: Materials in Electronics 2015, 27, 1014-1019.

3-56. Livingstone, R.; Zhou, X.; Tamargo, M.; Lombardi, J. R., Surface Enhanced Raman Spectroscopy of Pyridine on Cdse-Znbese Quantum Dots Grown by Molecular Beam Epitaxy. J. Phys. Chem. C 2010, 114, 4. 
3-57. Ding, T.; Sigle, D. O.; Herrmann, L. O.; Wolverson, D.; Baumberg, J. J., Nanoimprint Lithography of Al Nanovoids for Deep-Uv Sers. ACS applied materials \& interfaces 2014, 6, 17358-63.

3-58. Lee, Y.; Lee, J.; Lee, T. K.; Park, J.; Ha, M.; Kwak, S. K.; Ko, H., Particle-on-Film Gap Plasmons on Antireflective Zno Nanocone Arrays for Molecular-Level Surface-Enhanced Raman Scattering Sensors. ACS applied materials \& interfaces 2015, 7, 26421-9.

3-59. Bi, L.; Rao, Y.; Tao, Q.; Dong, J.; Su, T.; Liu, F.; Qian, W., Fabrication of Large-Scale Gold Nanoplate Films as Highly Active Sers Substrates for Label-Free DNA Detection. Biosensors and Bioelectronics 2013, 43, 193-199.

3-60. Mondal, S.; Rana, U.; Malik, S., Facile Decoration of Polyaniline Fiber with Ag Nanoparticles for Recyclable Sers Substrate. ACS applied materials \& interfaces 2015, 7, 10457 65.

3-61. Indrasekara, A. S. D. S.; Thomas, R.; Fabris, L., Plasmonic Properties of Regiospecific Core-Satellite Assemblies of Gold Nanostars and Nanospheres. Phys Chem Chem Phys 2015, 17, 9.

3-62. Yüksel, S.; Ziegler, M.; Goerke, S.; Hübner, U.; Pollok, K.; Langenhorst, F.; Weber, K.; Cialla-May, D.; Popp, J., Background-Free Bottom-up Plasmonic Arrays with Increased Sensitivity, Specificity and Shelf Life for Sers Detection Schemes. The Journal of Physical Chemistry C 2015, 119, 13791-13798.

3-63. Zhang, L.; Guan, C.; Wang, Y.; Liao, J., Highly Effective and Uniform Sers Substrates Fabricated by Etching Multi-Layered Gold Nanoparticle Arrays. Nanoscale 2016, 8, 5928-37.

3-64. Powell, J. A.; Venkatakrishnan, K.; Tan, B., Programmable Sers Active Substrates for Chemical and Biosensing Applications Using Amorphous/Crystalline Hybrid Silicon Nanomaterial. Scientific reports 2016, 6, 19663.

3-65. Wang, X.; Shi, W.; She, G.; Mu, L., Using Si and Ge Nanostructures as Substrates for Surface-Enhanced Raman Scattering Based on Photoinduced Charge Transfer Mechanism. Journal of the American Chemical Society 2011, 133, 16518-23. 
3-66. Khorasaninejad, M.; Walia, J.; Saini, S. S., Enhanced First-Order Raman Scattering from Arrays of Vertical Silicon Nanowires. Nanotechnology 2012, 23, 275706.

3-67. Cao, L.; Nabet, B.; Spanier, J. E., Enhanced Raman Scattering from Individual Semiconductor Nanocones and Nanowires. Phys Rev Lett 2006, 96, 157402.

3-68. Formo, E. V.; Mahurin, S. M.; Dai, S., Robust Sers Substrates Generated by Coupling a Bottom-up Approach and Atomic Layer Deposition. ACS applied materials \& interfaces 2010, 2, 1987-1991.

3-69. Mir-Simon, B.; Reche-Perez, I.; Guerrini, L.; Pazos-Perez, N.; Alvarez-Puebla, R. A., Universal One-Pot and Scalable Synthesis of Sers Encoded Nanoparticles. Chemistry of Materials 2015, 27, 950-958.

3-70. Premnath, P.; Tan, B.; Venkatakrishnan, K., Engineering Functionalized Multi-Phased Silicon/Silicon Oxide Nano-Biomaterials to Passivate the Aggressive Proliferation of Cancer. Scientific reports 2015, 5, 12141.

3-71. Chinnakkannu Vijayakumar, C.; Venkatakrishnan, K.; Tan, B., Harmonizing Hela Cell Cytoskeleton Behavior by Multi-Ti Oxide Phased Nanostructure Synthesized through Ultrashort Pulsed Laser. Scientific reports 2015, 5, 15294.

3-72. Chinnakkannu Vijayakumar, S.; Venkatakrishnan, K.; Tan, B., Manipulating Mammalian Cell by Phase Transformed Titanium Surface Fabricated through Ultra-Short Pulsed Laser Synthesis. Exp Cell Res 2016, 340, 274-82.

3-73. Thakur, P.; Tan, B.; Venkatakrishnan, K., Multi-Phase Functionalization of Titanium for Enhanced Photon Absorption in the Vis-Nir Region. Scientific reports 2015, 5, 15354.

3-74. Maznichenko, D.; Venkatakrishnan, K.; Tan, B., Stimulating Multiple Sers Mechanisms by a Nanofibrous Three-Dimensional Network Structure of Titanium Dioxide (Tio2). The Journal of Physical Chemistry C 2013, 117, 578-583.

3-75. Maznichenko, D.; Selvaganapathy, P. R.; Venkatakrishnan, K.; Tan, B., Tio2 Nanofibrous Interface Development for Raman Detection of Environmental Pollutants. Applied Physics Letters 2012, 101, 231602. 
3-76. Fan, M.; Andrade, G. F.; Brolo, A. G., A Review on the Fabrication of Substrates for Surface Enhanced Raman Spectroscopy and Their Applications in Analytical Chemistry. Anal Chim Acta 2011, 693, 7-25.

3-77. Que, R.; Shao, M.; Zhuo, S.; Wen, C.; Wang, S.; Lee, S.-T., Highly Reproducible SurfaceEnhanced Raman Scattering on a Capillarity-Assisted Gold Nanoparticle Assembly. Advanced Functional Materials 2011, 21, 3337-3343.

3-78. Ghosh, S.; Saha, M.; Dev Ashok, V.; Dalal, B.; De, S. K., Tunable Surface Plasmon Resonance in Sn-Doped Zn-Cd-O Alloyed Nanocrystals. The Journal of Physical Chemistry C 2015, 119, 1180-1187.

3-79. Cong, S., et al., Noble Metal-Comparable Sers Enhancement from Semiconducting Metal Oxides by Making Oxygen Vacancies. Nat Commun 2015, 6, 7800.

3-80. Lee, J.; Govorov, A. O.; Dulka, J.; Kotov, N. A., Bioconjugates of Cdte Nanowires and Au Nanoparticles: Plasmon-Exciton Interactions, Luminescence Enhancement, and Collective Effects. Nano Lett 2004, 4, 7.

3-81. Setti, G. O.; Mamián-López, M. B.; Pessoa, P. R.; Poppi, R. J.; Joanni, E.; Jesus, D. P., Sputtered Gold-Coated Ito Nanowires by Alternating Depositions from Indium and Ito Targets for Application in Surface-Enhanced Raman Scattering. Applied Surface Science 2015, 347, 17-22.

3-82. Yan, W.; Yang, L.; Zhuang, H.; Wu, H.; Zhang, J., Engineered "Hot" Core-Shell Nanostructures for Patterned Detection of Chloramphenicol. Biosensors \& bioelectronics 2016, $78,67-72$.

3-83. Guzel, R.; Ustundag, Z.; Eksi, H.; Keskin, S.; Taner, B.; Durgun, Z. G.; Turan, A. A.; Solak, A. O., Effect of Au and Au@Ag Core-Shell Nanoparticles on the Sers of Bridging Organic Molecules. J Colloid Interface Sci 2010, 351, 35-42.

3-84. Merga, G.; Saucedo, N.; Cass, L. C.; Puthussery, J.; Meisel, D., Naked Gold NanoparticlesSynthesis, Characterization, Catalytic Hydrogen Evolution, and Sers. J. Phys. Chem. C 2010, 114, 7.

3-85. Kneipp, K.; Kneipp, H.; Itzkan, I.; Dasari, R. R.; Feld, M. S., Surface-Enhanced Raman Scattering and Biophysics. Journal of Physics-Condensed Matter 2002, 14, R597-R624. 
3-86. Liu, H.; Yang, L.; Liu, J., Three-Dimentional Sers Hotspots for Chemical Sensing: Towards a Practical Analyzer. TrAC Trends in Analytical Chemistry 2015.

3-87. Khlebtsov, B.; Panfilova, E.; Khanadeev, V.; Khlebtsov, N., Improved Size-Tunable Synthesis and Sers Properties of Au Nanostars. Journal of Nanoparticle Research 2014, 16.

3-88. Chong, N. S.; Donthula, K.; Davies, R. A.; Ilsley, W. H.; Ooi, B. G., Significance of Chemical Enhancement Effects in Surface-Enhanced Raman Scattering (Sers) Signals of Aniline and Aminobiphenyl Isomers. Vibrational Spectroscopy 2015, 81, 22-31.

3-89. Stiles, P. L.; Dieringer, J. A.; Shah, N. C.; Van Duyne, R. P., Surface-Enhanced Raman Spectroscopy. Annu Rev Anal Chem (Palo Alto Calif) 2008, 1, 601-26.

3-90. Lombardi, J. R.; Birke, R. L., Time-Dependent Picture of the Charge-Transfer Contributions to Surface Enhanced Raman Spectroscopy. J Chem Phys 2007, 126, 244709.

3-91. Cho, D.; Kim, M.; Hwang, J.; Park, J. H.; Joo, Y. L.; Jeong, Y., Facile Synthesis of Porous Silicon Nanofibers by Magnesium Reduction for Application in Lithium Ion Batteries. Nanoscale Res Lett 2015, 10, 424.

3-92. Zhao, Y.; Zhang, Y.-L.; Huang, J.-A.; Zhang, Z.; Chen, X.; Zhang, W., Plasmonic Nanopillar Array Embedded Microfluidic Chips: An in Situ Sers Monitoring Platform. J. Mater. Chem. A 2015, 3, 5.

3-93. Liu, M.; Sun, L.; Cheng, C.; Hu, H.; Shen, Z.; Fan, H. J., Highly Effective Sers Substrates Based on an Atomic-Layer-Deposition-Tailored Nanorod Array Scaffold. Nanoscale 2011, 3, 3627-30.

3-94. Jin, M.; Pully, V.; Otto, C.; van den Berg, A.; Carlen, E. T., High-Density Periodic Arrays of Self-Aligned Subwavelength Nanopyramids for Surface-Enhanced Raman Spectroscopy. J. Phys. Chem. C 2010, 114, 6.

4-1. Ma, Q.; Nakazato, K., Low-Temperature Fabrication of Zno Nanorods/FerrocenylAlkanethiol Bilayer Electrode and Its Application for Enzymatic Glucose Detection. Biosensors \& bioelectronics 2014, 51, 362-5. 
4-2. Chauhan, R.; Singh, J.; Solanki, P. R.; Manaka, T.; Iwamoto, M.; Basu, T.; Malhotra, B. D., Label-Free Piezoelectric Immunosensor Decorated with Gold Nanoparticles: Kinetic Analysis and Biosensing Application. Sensors and Actuators B: Chemical 2016, 222, 804-814.

4-3. Uddin, R.; Burger, R.; Donolato, M.; Fock, J.; Creagh, M.; Hansen, M. F.; Boisen, A., Labon-a-Disc Agglutination Assay for Protein Detection by Optomagnetic Readout and Optical Imaging Using Nano- and Micro-Sized Magnetic Beads. Biosensors \& bioelectronics 2016, 85, $351-7$.

4-4. Chen, F.; Flaherty, B. R.; Cohen, C. E.; Peterson, D. S.; Zhao, Y., Direct Detection of Malaria Infected Red Blood Cells by Surface Enhanced Raman Spectroscopy. Nanomedicine : nanotechnology, biology, and medicine 2016, 12, 1445-51.

4-5. Wang, Y.; Irudayaraj, J., Surface-Enhanced Raman Spectroscopy at Single-Molecule Scale and Its Implications in Biology. Philosophical transactions of the Royal Society of London. Series B, Biological sciences 2013, 368, 20120026.

4-6. Xiao, R.; Wang, C. W.; Zhu, A. N.; Long, F., Single Functional Magnetic-Bead as Universal Biosensing Platform for Trace Analyte Detection Using Sers-Nanobioprobe. Biosensors \& bioelectronics 2016, 79, 661-8.

4-7. Oo, S. Z., et al., A Nanoporous Gold Membrane for Sensing Applications. Sensing and Bio-Sensing Research 2016, 7, 133-140.

4-8. Wallace, G. Q.; Zuin, M. S.; Tabatabaei, M.; Gobbo, P.; Lagugne-Labarthet, F.; Workentin, M. S., Gold Nanosponges (Auns): A Versatile Nanostructure for Surface-Enhanced Raman Spectroscopic Detection of Small Molecules and Biomolecules. The Analyst 2015, 140, 7278-82.

4-9. Zheng, X.; Fu, Q.; Guo, H.; Wu, M.; Lei, Y., Sensitive and Reproducible Nanogap Sers Substrates for Glucose Biosensing. Nanomedicine : nanotechnology, biology, and medicine 2016, 12.

4-10. Wang, H. N.; Fales, A. M.; Vo-Dinh, T., Plasmonics-Based Sers Nanobiosensor for Homogeneous Nucleic Acid Detection. Nanomedicine : nanotechnology, biology, and medicine 2015, 11, 811-4. 
4-11. Bizzarri, A. R.; Cannistraro, S., Sers Detection of Thrombin by Protein Recognition Using Functionalized Gold Nanoparticles. Nanomedicine : nanotechnology, biology, and medicine 2007, 3, 306-10.

4-12. Yao, Q.; Zhang, Z.; Cao, F.; Feng, C.; Zhao, Y.; Wang, X., Targeting, Sers Detection and Growth Inhibition of Lymphoma Cells Using Functionalized Ag Nanoparticles. Nanomedicine : nanotechnology, biology, and medicine 2016, 12, 1.

4-13. Convertino, A.; Mussi, V.; Maiolo, L., Disordered Array of Au Covered Silicon Nanowires for Sers Biosensing Combined with Electrochemical Detection. Scientific reports 2016, 6, 25099.

4-14. Li, T.; Wu, K.; Rindzevicius, T.; Wang, Z.; Schulte, L.; Schmidt, M. S.; Boisen, A.; Ndoni, S., Wafer-Scale Nanopillars Derived from Block Copolymer Lithography for Surface-Enhanced Raman Spectroscopy. ACS applied materials \& interfaces 2016, 8, 15668-75.

4-15. Zhang, C.; Man, B. Y.; Jiang, S. Z.; Yang, C.; Liu, M.; Chen, C. S.; Xu, S. C.; Qiu, H. W.; Li, Z., Sers Detection of Low-Concentration Adenosine by Silver Nanoparticles on Silicon Nanoporous Pyramid Arrays Structure. Applied Surface Science 2015, 347, 668-672.

4-16. Zhang, C., et al., Gold@ Silver Bimetal Nanoparticles/Pyramidal Silicon 3d Substrate with High Reproducibility for High-Performance Sers. Scientific reports 2016, 6, 25243.

4-17. Yang, L.; Gong, M.; Jiang, X.; Chen, Y.; Han, X.; Song, K.; Sun, X.; Zhang, Y.; Zhao, B., Sers Investigation and Detection of Levofloxacin Drug Molecules on Semiconductor Tio2: Charge Transfer Contribution. Colloids and Surfaces A: Physicochemical and Engineering Aspects 2016, 508, 142-149.

4-18. Chang, L.; Xu, D.; Xue, X., Photoluminescence and Raman Scattering Study in Zno:Mg Nanocrystals. Journal of Materials Science: Materials in Electronics 2015, 27, 1014-1019.

4-19. Lombardi, J. R.; Birke, R. L., Theory of Surface-Enhanced Raman Scattering in Semiconductors. The Journal of Physical Chemistry C 2014, 118, 11120-11130.

4-20. Yang, L.; Gong, M.; Jiang, X.; Yin, D.; Qin, X.; Zhao, B.; Ruan, W., Investigation on Sers of Different Phase Structure Tio2nanoparticles. Journal of Raman Spectroscopy 2015, 46, 287292. 
4-21. Zeng, X.; Yan, S.; Cui, J.; Liu, H.; Dong, J.; Xia, W.; Zhou, M.; Chen, H., Size- and Morphology-Dependent Optical Properties of Zns:Al One-Dimensional Structures. Journal of nanoparticle research : an interdisciplinary forum for nanoscale science and technology 2015,17 , 188.

4-22. Mao, H.; Chen, Y.; Wang, J., Raman Scattering and Luminescence Emission of the Cdse/Zns Quantum Dots Mediated by the Surface Plasmon. Optical and Quantum Electronics 2015, 47, 2811-2819.

4-23. Zhang, R.; Yin, P.-G.; Wang, N.; Guo, L., Photoluminescence and Raman Scattering of Zno Nanorods. Solid State Sciences 2009, 11, 865-869.

4-24. Novara, C., et al., Sers-Active Ag Nanoparticles on Porous Silicon and Pdms Substrates: A Comparative Study of Uniformity and Raman Efficiency. The Journal of Physical Chemistry C 2016, 120, 16946-16953.

4-25. Powell, J. A.; Venkatakrishnan, K.; Tan, B., Programmable Sers Active Substrates for Chemical and Biosensing Applications Using Amorphous/Crystalline Hybrid Silicon Nanomaterial. Scientific reports 2016, 6, 19663.

4-26. Powell, J. A.; Venkatakrishnan, K.; Tan, B., Hybridized Enhancement of the Sers Detection of Chemical and Bio-Marker Molecules through Au Nanosphere Ornamentation of Hybrid Amorphous/Crystalline Si Nanoweb Nanostructure Biochip Devices. J Mater Chem B 2016, 4, 5713-5728.

4-27. Zhang, H.; Wang, C.; Wang, K.; Xuan, X.; Lv, Q.; Jiang, K., Ultrasensitive Fluorescent Ratio Imaging Probe for the Detection of Glutathione Ultratrace Change in Mitochondria of Cancer Cells. Biosensors \& bioelectronics 2016, 85, 96-102.

4-28. Xu, K.; Thornalley, P. J., Involvement of Glutathione Metabolism in the Cytotoxicity of the Phenethyl Isothiocyanate and Its Cysteine Conjugate to Human Leukaemia Cells in Vitro. Biochemical Pharmacology 2001, 61, 12.

4-29. Hong, S.; Li, X., Optimal Size of Gold Nanoparticles for Surface-Enhanced Raman Spectroscopy under Different Conditions. Journal of Nanomaterials 2013, 2013, 1-9. 
4-30. Wang, J., et al., Facile Synthesis of Au-Coated Magnetic Nanoparticles and Their Application in Bacteria Detection Via a Sers Method. ACS applied materials \& interfaces 2016, 8, 19958-67.

4-31. Xu, J. Q.; Duo, H. H.; Zhang, Y. G.; Zhang, X. W.; Fang, W.; Liu, Y. L.; Shen, A. G.; Hu, J. M.; Huang, W. H., Photochemical Synthesis of Shape-Controlled Nanostructured Gold on Zinc Oxide Nanorods as Photocatalytically Renewable Sensors. Analytical chemistry 2016, 88, 378995.

4-32. Hong, X.; Chu, X.; Zou, P.; Liu, Y.; Yang, G., Magnetic-Field-Assisted Rapid Ultrasensitive Immunoassays Using Fe3o4/Zno/Au Nanorices as Raman Probes. Biosensors \& bioelectronics 2010, 26, 918-22.

4-33. Lombardi, J. R.; Birke, R. L., Time-Dependent Picture of the Charge-Transfer Contributions to Surface Enhanced Raman Spectroscopy. J Chem Phys 2007, 126, 244709.

4-34. Stiles, P. L.; Dieringer, J. A.; Shah, N. C.; Van Duyne, R. P., Surface-Enhanced Raman Spectroscopy. Annu Rev Anal Chem (Palo Alto Calif) 2008, 1, 601-26.

4-35. Bains, J. S.; Shaw, C. A., Neurodegenerative Disorders in Humans the Role of Glutathione in Oxidative Stress-Mediated Neuronal Death. Brain Research Reviews 1997, 25, 23.

4-36. Brambilla, A.; Philippidis, A.; Nevin, A.; Comelli, D.; Valentini, G.; Anglos, D., Adapting and Testing a Portable Raman Spectrometer for Sers Analysis of Amino Acids and Small Peptides. Journal of Molecular Structure 2013, 1044, 121-127.

4-37. Cho, D.; Kim, M.; Hwang, J.; Park, J. H.; Joo, Y. L.; Jeong, Y., Facile Synthesis of Porous Silicon Nanofibers by Magnesium Reduction for Application in Lithium Ion Batteries. Nanoscale Res Lett 2015, 10, 424.

4-38. Qin, L.; Li, X.; Kang, S. Z.; Mu, J., Gold Nanoparticles Conjugated Dopamine as Sensing Platform for Sers Detection. Colloids and surfaces. B, Biointerfaces 2015, 126, 210-6.

4-39. Hong, D.-Y.; Kim, S. K.; Kwon, Y.-U., Synergistic Effects between Gold Nanoparticles and Nanostructured Platinum Film in Surface-Enhanced Raman Spectroscopy. The Journal of Physical Chemistry C 2015, 119, 22611-22617. 
5-1. G. Liu, Y. Li, G. Duan, J. Wang, Changhao, Liang and W. Cai, ACS applied materials \& interfaces, 2012, 4, 1-5.

5-2. P. Yang, J. Zheng, Y. Xu, Q. Zhang and L. Jiang, Advanced materials, 2016, 28, 1050810517.

5-3. H. J. Ahn, P. Thiyagarajan, L. Jia, S. I. Kim, J. C. Yoon, E. L. Thomas and J. H. Jang, Nanoscale, 2013, 5, 1836-1842.

5-4. C. Wang, X. Wu, P. Dong, J. Chen and R. Xiao, Biosensors \& bioelectronics, 2016, 86, 944-950.

5-5. B. Fazio, C. D'Andrea, A. Foti, E. Messina, A. Irrera, M. G. Donato, V. Villari, N. Micali, O. M. Marago and P. G. Gucciardi, Scientific reports, 2016, 6, 26952.

5-6. Q. Zhang, L. Han, H. Jing, D. A. Blom, Y. Lin, H. L. Xin and H. Wang, ACS Nano, 2016, 10, 2960-2974.

5-7. Y. Huang, L. Dai, L. Song, L. Zhang, Y. Rong, J. Zhang, Z. Nie and T. Chen, ACS applied materials \& interfaces, 2016, DOI: 10.1021/acsami.6b05258.

5-8. N. Wiriyakun, K. Pankhlueab, S. Boonrungsiman and R. Laocharoensuk, Scientific reports, 2016, 6, 39115 .

5-9. M. Focsan, A. Campu, A. M. Craciun, M. Potara, C. Leordean, D. Maniu and S. Astilean, Biosensors \& bioelectronics, 2016, 86, 728-735.

5-10. R. Xiao, X. Zhang, Z. Rong, B. Xiu, X. Yang, C. Wang, W. Hao, Q. Zhang, Z. Liu, C. Duan, K. Zhao, X. Guo, Y. Fan, Y. Zhao, H. Johnson, Y. Huang, X. Feng, X. Xu, H. Zhang and S. Wang, Nanomedicine : nanotechnology, biology, and medicine, 2016, 12, 2475-2484.

5-11. A. Chiado, C. Novara, A. Lamberti, F. Geobaldo, F. Giorgis and P. Rivolo, Analytical chemistry, 2016, 88, 9554-9563.

5-12. J. Huang, D. Ma, F. Chen, D. Chen, M. Bai, K. Xu and Y. Zhao, ACS applied materials \& interfaces, 2017, DOI: 10.1021/acsami.6b14571.

5-13. N. Bontempi, L. Carletti, C. De Angelis and I. Alessandri, Nanoscale, 2016, 8, 3226-3231. 
5-14. Y. Tan, J. Gu, W. Xu, Z. Chen, D. Liu, Q. Liu and D. Zhang, ACS applied materials \& interfaces, 2013, 5, 9878-9882.

5-15. A. G. Milekhin, L. L. Sveshnikova, T. A. Duda, N. A. Yeryukov, E. E. Rodyakina, A. K. Gutakovskii, S. A. Batsanov, A. V. Latyshev and D. R. T. Zahn, Physica E: Low-dimensional Systems and Nanostructures, 2016, 75, 210-222.

5-16. J. A. Powell, K. Venkatakrishnan and B. Tan, Scientific reports, 2016, 6, 19663.

5-17. J.-F. Bryche, B. Bélier, B. Bartenlian and G. Barbillon, Sensors and Actuators B: Chemical, 2017, 239, 795-799.

5-18. Q. Zhao, G. Liu, H. Zhang, F. Zhou, Y. Li and W. Cai, Journal of Hazardous Materials, 2017, 324, 194-202.

5-19. L. Zhang, H. Li, G. Chu, L. Luo, J. Jin, B. Zhao and Y. Tian, Colloids and Surfaces A: Physicochemical and Engineering Aspects, 2016, 508, 309-315.

5-20. J. R. Lombardi and R. L. Birke, The Journal of Physical Chemistry C, 2014, 118, 1112011130.

5-21. H. Wei, M. Wu, Z. Dong, Y. Chen, J. Bu, J. Lin, Y. Yu, Y. Wei, Y. Cui and R. Wang, Journal of Raman Spectroscopy, 2017, DOI: 10.1002/jrs.5080.

5-22. C. Muehlethaler, C. R. Considine, V. Menon, W.-C. Lin, Y.-H. Lee and J. R. Lombardi, ACS Photonics, 2016, 3, 1164-1169.

5-23. S. Cong, Y. Yuan, Z. Chen, J. Hou, M. Yang, Y. Su, Y. Zhang, L. Li, Q. Li, F. Geng and Z. Zhao, Nat Commun, 2015, 6, 7800 .

5-24. X. Zhang, Z. Yu, W. Ji, H. Sui, Q. Cong, X. Wang and B. Zhao, The Journal of Physical Chemistry C, 2015, 119, 22439-22444.

5-25. C. D'Andrea, M. J. Faro, G. Bertino, P. M. Ossi, F. Neri, S. Trusso, P. Musumeci, M. Galli, N. Cioffi, A. Irrera, F. Priolo and B. Fazio, Nanotechnology, 2016, 27, 375603.

5-26. X. Liu, S. Lebedkin, H. Besser, W. Pfleging, S. Prinz, M. Wissmann, P. M. Schwab, I. Nazarenko, M. Guttmann, M. M. Kappes and U. Lemmer, Acs Nano, 2015, 9, 260-270. 
5-27. L. Mikac, M. Ivanda, V. Đerek and M. Gotić, Journal of Raman Spectroscopy, 2016, 47, 1036-1041.

5-28. D. Lin, Z. Wu, S. Li, W. Zhao, C. Ma, J. Wang, Z. Jiang, Z. Zhong, Y. Zheng and X. Yang, ACS Nano, 2017, DOI: 10.1021/acsnano.6b06778.

5-29. C. Zhang, S. Z. Jiang, C. Yang, C. H. Li, Y. Y. Huo, X. Y. Liu, A. H. Liu, Q. Wei, S. S. Gao, X. G. Gao and B. Y. Man, Scientific reports, 2016, 6, 25243.

5-30. P. P. Zhang, J. Gao and X. H. Sun, Applied Physics Letters, 2015, 106, 043103.

5-31. J. A. Powell, K. Venkatakrishnan and B. Tan, J Mater Chem B, 2016, 4, 5713-5728.

5-32. H. Wu, H. Wang and G. Li, The Analyst, 2017, 142, 326-335.

5-33. W. Ji, Y. Wang, I. Tanabe, X. X. Han, B. Zhao and Y. Ozaki, Chemical Science, 2015, 6, $342-348$.

5-34. W. Qian and S. Krimm, Biopolymers, 1994, 34, 1377-1394.

5-35. C. A. Opitz, W. Wick, L. Steinman and M. Platten, Cellular and molecular life sciences : CMLS, 2007, 64, 2542-2563.

5-36. Y. Luo, L. Zhang, W. Liu, Y. Yu and Y. Tian, Angewandte Chemie, 2015, 54, 1405314056.

5-37. J. M. Mato, M. L. Martinez-Chantar and S. C. Lu, Annual review of nutrition, 2008, 28, 273-293.

5-38. P. P. Pronko, P. A. VanRompay, C. Horvath, F. Loesel, T. Juhasz, X. Liu and G. Mourou, Physical Review B, 1998, 58, 2387-2390.

5-39. X.-Y. Liu, J.-A. Huang, B. Yang, X.-J. Zhang and Y.-Y. Zhu, AIP Advances, 2015, 5, 057159 .

5-40. G. Seniutinas, G. Gervinskas, R. Verma, B. D. Gupta, F. Lapierre, P. R. Stoddart, F. Clark, S. L. McArthur and S. Juodkazis, Optics express, 2015, 23, 6763-6772.

5-41. C.-Y. Chen, L.-J. Hsu, P.-H. Hsiao and C.-T. R. Yu, Applied Surface Science, 2015, 355, 197-202. 
5-42. J. Zheng, B. Dai, J. Liu, J. Liu, M. Ji, J. Liu, Y. Zhou, M. Xu and J. Zhang, ACS applied materials \& interfaces, 2016, 8, 35426-35434.

5-43. H. Cheng, Y. Zhao, Y. Fan, X. Xie, L. Qu and G. Shi, ACS Nano, 2012, 6, 2237-2244.

5-44. P. Zaumseil, Journal of applied crystallography, 2015, 48, 528-532.

5-45. X. Xue, W. Ji, Z. Mao, H. Mao, Y. Wang, X. Wang, W. Ruan, B. Zhao and J. R. Lombardi, The Journal of Physical Chemistry C, 2012, 116, 8792-8797.

5-46. X. Wu, J. Yu, T. Ren and L. Liu, Microelectronics Journal, 2007, 38, 87-90.

5-47. L. P. Avakyants, L. L. Gerasimov, V. S. Gorelik, N. M. Manja, E. D. Obraztsova and Y. I. Plotnikov, Journal of Molecular Structure, 1992, 267, 177-184.

5-48. D. M. Townsend, K. D. Tew and H. Tapiero, Biomedicine \& Pharmacotherapy, 2003, 57, $145-155$.

5-49. G. G. Huang, X. X. Han, M. K. Hossain and Y. Ozaki, Analytical chemistry, 2009, 81, 5881-5888.

5-50. J. De Gelder, K. De Gussem, P. Vandenabeele and L. Moens, Journal of Raman Spectroscopy, 2007, 38, 1133-1147.

5-51. L. Li, T. Hutter, U. Steiner and S. Mahajan, The Analyst, 2013, 138, 4574-4578.

5-52. Y. Lee, J. Lee, T. K. Lee, J. Park, M. Ha, S. K. Kwak and H. Ko, ACS applied materials \& interfaces, 2015, 7, 26421-26429.

5-53. S. Chinnakkannu Vijayakumar, K. Venkatakrishnan and B. Tan, ACS applied materials \& interfaces, 2017, DOI: 10.1021/acsami.6b13576.

5-54. Y. Huang, S. Xiong, G. Liu and R. Zhao, Chemical communications, 2011, 47, 8319-8321.

5-55. Y. Liu, X. Lv, M. Hou, Y. Shi and W. Guo, Analytical chemistry, 2015, 87, 11475-11483.

5-56. E. Podstawka, Y. Ozaki and L. M. Proniewicz, Applied spectroscopy, 2004, 58, 570-580.

5-57. G. Diaz Fleming, J. J. Finnerty, M. Campos-Vallette, F. Célis, A. E. Aliaga, C. Fredes and R. Koch, Journal of Raman Spectroscopy, 2009, 40, 632-638. 
5-58. C.-H. Chuang and Y.-T. Chen, Journal of Raman Spectroscopy, 2009, 40, 150-156.

5-59. S. Pandiarajan, M. Umadevi, M. Briget Mary, R. K. Rajaram and V. Ramakrishnan, Journal of Raman Spectroscopy, 2004, 35, 907-913. 


\section{$\underline{\text { Appendix A }}$}

1. Published Articles

a. Powell, J.A., Venkatakrishnan, K., Tan, B. Programmable SERS active substrates for chemical and biosensing applications using amorphous/crystalline hybrid silicon nanomaterial (2016) Scientific Reports, 6, art. no. 19663 (Impact Factor: $5.228)$

b. Powell, J.A., Venkatakrishnan, K., Tan, B. Hybridized enhancement of the SERS detection of chemical and bio-marker molecules through Au nanosphere ornamentation of hybrid amorphous/crystalline Si nanoweb nanostructure biochip devices (2016) Journal of Materials Chemistry B, 4 (34), pp. 5713-5728 (Impact Factor: 4.872)

2. Submitted for Review

a. Powell, J.A., Venkatakrishnan, K., Tan, B. SERS-active interconnected Si nanocore network platforms for primary biomolecule detection with noble metal nanosatellites as secondary boosting mechanism Submitted to RCS Advances (Impact Factor: 3.289)

3. Pending Submission Upon Approval

a. Powell, J.A., Venkatakrishnan, K., Tan, B. Towards Universal Si SERS-based nanobioprobe materials through engineering quantum-scale defects within selfassembled Si-only disordered 3D nanomesh structures 